LBL-36026

UC-412

\title{
Correlation Function Analysis of the COBE Differential Microwave Radiometer Sky Maps
}

\author{
Charles Howe Lineweaver \\ Ph.D. Thesis \\ Space Sciences Laboratory \\ University of California \\ and \\ Physics Division \\ Lawrence Berkeley Laboratory \\ University of California \\ Berkeley, California 94720
}

August 1994

This work was supported in part by the Director, Office of Energy Research, Office of High Energy and Nuclear Physics, Division of High Energy Physics, of the U.S. Department of Energy under Contract No. DE-AC0376 SF00098 and by a National Aeronautics and Space Administration Research Training Grant (NGT-50783) and the University of California. 


\section{DISCLAIMER}

This report was prepared as an account of work sponsored by an agency of the United States Government. Neither the United States Government nor any agency thereof, nor any of their employees, make any warranty, express or implied, or assumes any legal liability or responsibility for the accuracy, completeness, or usefulness of any information, apparatus, product, or process disclosed, or represents that its use would not infringe privately owned rights. Reference herein to any specific commercial product, process, or service by trade name, trademark, manufacturer, or otherwise does not necessarily constitute or imply its endorsement, recommendation, or favoring by the United States Government or any agency thereof. The views and opinions of authors expressed herein do not necessarily state or reflect those of the United States Government or any agency thereof. 


\section{DISCLAIMER}

Portions of this document may be illegible in electronic image products. Images are produced from the best available original document. 


\begin{abstract}
Correlation Function Analysis of the COBE

Differential Microwave Radiometer Sky Maps
\end{abstract}

by

Charles Howe Lineweaver

Doctor of Philosophy in Physics

University of California at Berkeley

Professor George F. Smoot, Chair

The Differential Microwave Radiometer (DMR) aboard the COBE satellite has detected anisotropies in the cosmic microwave background (CMB) radiation. A two-point correlation function analysis which helped lead to this discovery is presented in detail. The results of a correlation function analysis of the two year DMR data set is presented. The first and second year data sets are compared and found to be reasonably consistent. The positive correlation for separation angles less than $\sim 20^{\circ}$ is robust to Galactic latitude cuts and is very stable from year to year. The Galactic latitude cut independence of the correlation function is strong evidence that the signal is not Galactic in origin. The statistical significance of the structure seen in the correlation function of the first, second and two year maps is respectively $>9 \sigma,>10 \sigma$ and $>18 \sigma$ above the noise. The best $\chi^{2}$ fit of the the model power spectra parameters $n$ and $Q_{r m s-P S}$ to the correlation function yields $n=1.2_{-1.0}^{+0.6}$ and $Q_{r m s-P S}=17.3_{-5.1}^{+7.1}$.

The noise in the DMR sky maps is correlated at a low level. The structure of the pixel temperature covariance matrix is given. The noise covariance matrix of a DMR sky map is diagonal to an accuracy of better than $1 \%$. For a given sky pixel, the dominant noise covariance occurs with the ring of pixels at an angular separation of $60^{\circ}$ due to the $60^{\circ}$ separation of the DMR horns. The mean covariance at $60^{\circ}$ is $0.45 \%_{-0.14}^{+0.18}$ of the mean variance. The noise properties of the DMR maps are thus well approximated by the noise 
properties of maps made by a single-beam experiment. Previously published DMR results are not significantly affected by correlated noise. 


\section{Contents}

List of Figures vi

List of Tables viii

Thesis Summary ix

1 Cosmology and the Cosmic Microwave Background 1

1.1 The Standard Hót Big Bang Model . . . . . . . . . . . . 1

$1.2 \mathrm{CMB}$ and the Surface of Last Scattering . . . . . . . . . . . . 4

1.3 Anisotropy Mechanisms in a Perturbed Robertson-Walker Universe . . . . . 9

1.4 Structure Formation and Anisotropy Predictions . . . . . . . . . . 12

2 The DMR Experiment 14

2.1 COBE Mission . . . . . . . . . . . . . . . . 14

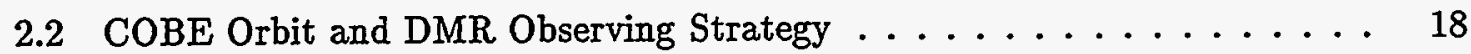

2.3 Galactic Foreground . . . . . . . . . . . . . . 18

2.4 Instrumentation. . . . . . . . . . . . . . . . 22

2.5 Noise . . . . . . . . . . . . . . . . . . . 22

3 Data Processing 25

3.1 Differential Data . . . . . . . . . . . . . . 25

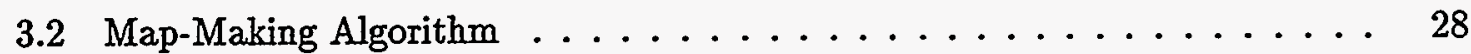

3.2.1 Pointing and Pixelization $\ldots \ldots \ldots \ldots \ldots \ldots \ldots \ldots \ldots$

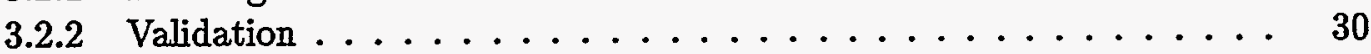

4 Correlation Function: Theory versus Practice 33

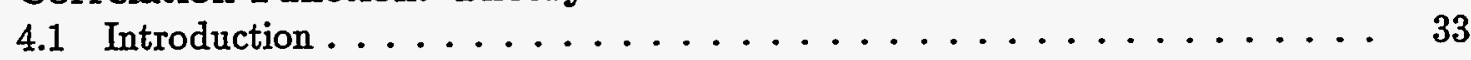

4.2 Legendre Polynomial Expansion . . . . . . . . . . . . . . . 34

4.3 Model Power Spectra . . . . . . . . . . . . . . . . . . 35

4.4 Cosmic Variance . . . . . . . . . . . . . . . . . . 38

4.5 Beam Smoothing . . . . . . . . . . . . . . 38

4.6 Correlation Function from DMR Maps . . . . . . . . . . . . 43

4.7 RMS Temperature Fluctuations from $C(\alpha) \ldots \ldots \ldots \ldots \ldots \ldots$

4.8 New Way to Estimate $Q_{r m s} \ldots \ldots \ldots \ldots \ldots \ldots \ldots \ldots$

4.9 Bias Due to Multipole Subtraction and Galactic Latitude Cuts . . . . . 46 
5 The First Year Results 48

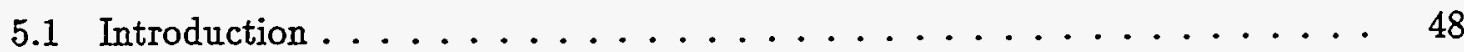

5.2 Is It Noise $\ldots \ldots \ldots \ldots \ldots \ldots \ldots \ldots$

5.3 Is It Galaxy? . . . . . . . . . . . . . . . . . . . . . 52

5.4 If It's CMB, How Large is the Signal? . . . . . . . . . . . . . . . . . . 54

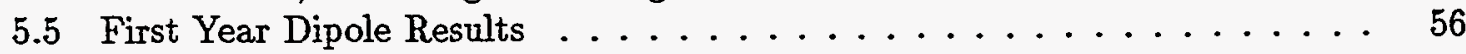

5.5.1 Calibrator of Other Diffuse Backgrounds . . . . . . . . . 57

6 Correlation Function Error Analysis 60

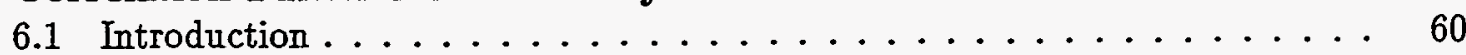

6.2 Error Bars and Covariance Matrices of $C(\alpha) \ldots \ldots \ldots 61$

6.3 Separation Angle, Galactic Cut and Time Dependence . . . . . . . . . 65

7 Correlation Function Analysis of the Two Year Maps 67

7.1 Channel Comparison, A+B and A-B Comparison . . . . . . . . . . 67

7.2 Galactic Latitude Independence . . . . . . . . . . . . . . . . . . . . . . 75

7.3 RMS Temperature Fluctuations and $Q_{r m s} \ldots \ldots \ldots \ldots$

8 Correlation Function Comparison of the First and Second Year Maps 82

8.1 Year One vs Year Two Data . . . . . . . . . . . . . . . . . . . . 82

8.2 Channel Comparison, $\mathrm{A}+\mathrm{B}$ vs $\mathrm{A}-\mathrm{B}$ Comparison . . . . . . . . . 83

8.3 Statistical Significance and Galactic Cuts ............... 88

9 Correlated Noise in the Maps 92

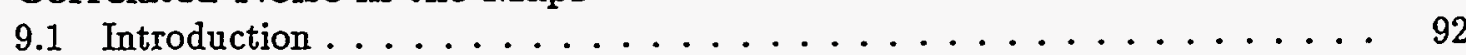

9.2 Pixel Covariance Matrix $\mathrm{A}^{-1} \ldots \ldots \ldots \ldots \ldots \ldots$

9.3 Correlated Noise Estimates ... . . . . . . . . . . . . . . 96

9.3.1 Correlated Noise Estimates from the Covariance Matrix . . . . . . . 96

9.3.2 Correlated Noise Estimates from Monte Carlo Simulations . . . . . . 97

9.3.3 Correlated Noise Estimates from DMR Maps . . . . . . . . . . 100

9.4 Cosmological Implications . . . . . . . . . . . . . . . . . . 100

9.5 Summary ... . . . . . . . . . . . . . . . . . 102

10 Estimates of $n$ and $Q_{r m s-P S}$ from the Correlation Function 103

10.1 The $n$ and $Q_{r m s-P S}$ Parametrization of the Power Spectrum . . . . . . 103

$10.2 \chi^{2}$ fitting for $n$ and $Q_{r m s-P S} \ldots \ldots \ldots \ldots \ldots \ldots$

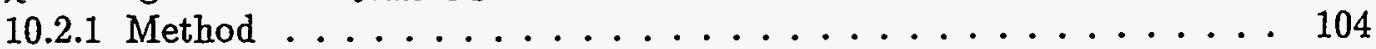

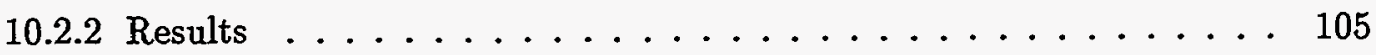

10.3 Other Determinations of $n$ and $Q_{r m s-P S} \ldots \ldots \ldots \ldots$. . . . . . . . . .

11 Conclusions and the Future 111

11.1 Conclusions . . . . . . . . . . . . . . . . . 111

11.2 Further DMR Analysis . . . . . . . . . . . . . . . . . . . . . 112

11.3 Experimental Assault on CMB Anisotropies . . . . . . . . . . . . . . . 112

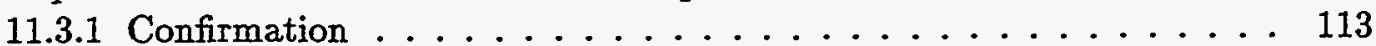

11.3.2 Small Angular Scales . . . . . . . . . . . . . . . 113 
11.4 New Field of Astronomy . . . . . . . . . . . . . . . . . 114

Bibliography

Appendices

$\begin{array}{ll}\text { A DMR Publications } & 125\end{array}$

$\begin{array}{ll}\text { B Thesis Notation } & 129\end{array}$

C DMR Notation $\quad 131$

D Literature Notation $\quad 133$

E Software Versions: Pass 1 vs Pass 2

$\begin{array}{ll}\text { F Friedmann Equation } & 139\end{array}$

G Antenna Temperature 140

H Spherical Harmonic Coefficients $a_{\ell m}$ and $b_{\ell m}$

I Correlation Function Power Spectrum Expansion $\quad 145$

J Arbitrary Mean Value of Map Solution $\quad 147$

K Error on the Correlation Function $\quad 150$

L Relation to Density Contrast Two-Point Correlation Function 153

$\begin{array}{ll}\text { Acknowledgements } & 155\end{array}$ 


\section{List of Figures}

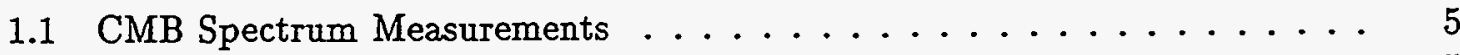

1.2 CMB Isotropy . . . . . . . . . . . . . . . . . . . 5

1.3 Space-Time and the Surface of Last Scattering . . . . . . . . . . . . . . 6

1.4 Monopole, Dipole and Structure ................. 11

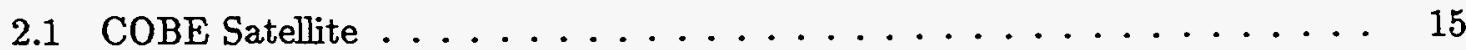

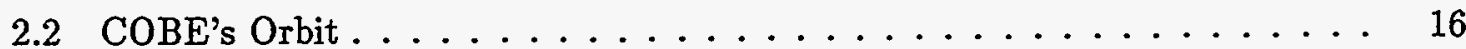

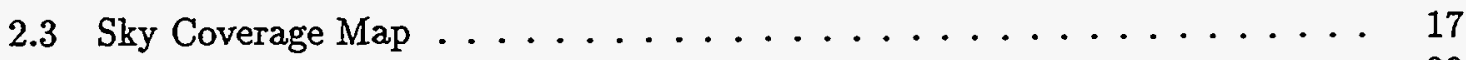

2.4 Galactic Foregrounds . . . . . . . . . . . . . . . . . . . . . 20

2.5 DMR Heterodyne Receiver . . . . . . . . . . . . . 21

3.1 Effect of Noise Spike on the Map Solution: 1st Iteration . . . . . . . . . . . . . 31

3.2 Effect of Noise Spike on the Map Solution: 2nd Iteration . . . . . . . . . 31

3.3 Effect of Noise Spike on the Map Solution: 5 th Iteration . . . . . . . . . . . . . 32

3.4 Effect of Noise Spike on the Map Solution: 30 th Iteration . . . . . . . . 32

4.1 Legendre Polynomial Expansion of Correlation Function . . . . . . . . . . . 36

4.2 Degeneracy of $n$ and $Q_{r m s-P S} \ldots \ldots \ldots \ldots \ldots \ldots \ldots$

4.3 Cosmic Variance . . . . . . . . . . . . . . . . . . . . 40

4.4 Beam Smoothing of Power . . . . . . . . . . . . . . . . . . . . . . 42

4.5 Correlation Functions of Dipole and Galaxy . . . . . . . . . . . . . . . . 44

4.6 Auto-correlation Multipole Subtraction Bias . . . . . . . . . . . . . 47

5.1 Yr1 Separate Channels . . . . . . . . . . . . . . . . . . . 50

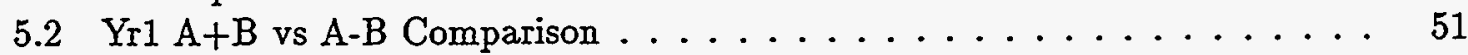

$5.3 \mathrm{Yr} 153 \mathrm{GHz}$ Galactic Latitude Cut Independence . . . . . . . . . . . . 53

5.4 Yr1 Galactic Latitude Cut Independence . . . . . . . . . . . . . . . . . . 53

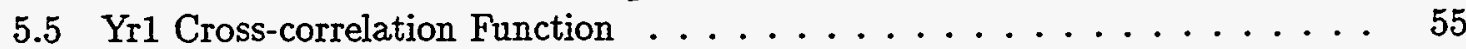

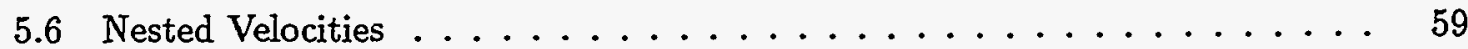

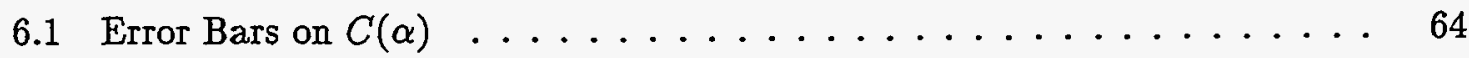

7.12 Yr Separate Channel Comparison . . . . . . . . . . . . . . . . 68

$7.22 Y_{\mathrm{I}} \mathrm{A}+\mathrm{B}$ vs A-B Comparison . . . . . . . . . . . . . . . . . . 69

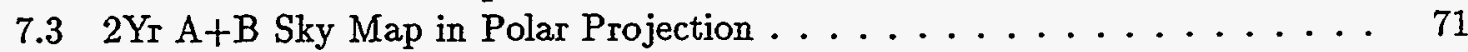

7.4 2Yr A-B Sky Maps in Polar Projection . . . . . . . . . . . . . . 72 
$7.52 \mathrm{Yr}$ Best Combinations $\mathrm{A}+\mathrm{B}$ and $\mathrm{A}-\mathrm{B} \ldots \ldots \ldots \ldots \ldots \ldots \ldots$

7.6 2Yr Cross-correlation of $53 \mathrm{GHz}$ with $90 \mathrm{GHz} \ldots \ldots \ldots 74$

$7.72 \mathrm{Yr}_{\mathrm{r}}$ Galactic Latitude Cut Independence . . . . . . . . . . . . . . . . . . . . 77

7.8 Frequency Dependence of the Galactic Plane . . . . . . . . . . . . . 78

$7.92 Y_{r}$ Correlation Functions with and without Quadrupole . . . . . . . 81

8.1 Yr1 vs $Y_{r} 2$ Separate Channel Comparison . . . . . . . . . . . . . 84

8.2 Yr1 vs $\mathrm{Yr}_{\mathrm{r} 2} \mathrm{~A}+\mathrm{B}$ vs $\mathrm{A}-\mathrm{B}$ Comparison $\ldots \ldots \ldots \ldots \ldots \ldots$

8.3 Correlation Function Comparison of $\mathrm{Y}_{\mathrm{r} 1}, \mathrm{Yr}_{\mathrm{r} 2}$ and $2 \mathrm{Y}_{\mathrm{r}} \ldots \ldots \ldots \ldots$

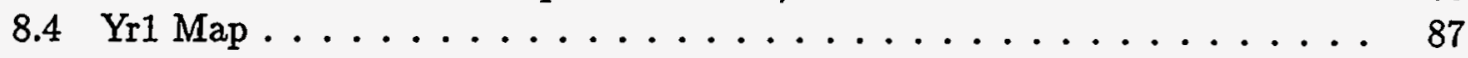

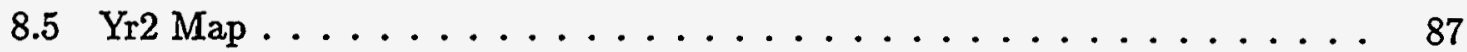

8.6 Yr1 Galactic Latitude Cut Independence . . . . . . . . . . . . . . . 90

8.7 Yr2 Galactic Latitude Cut Independence . . . . . . . . . . . . . . . . 90

8.8 Yr1 and Yr2 Correlation Functions with and without Quadrupole . . . . . 91

9.1 Covariance Matrix Inverse A . . . . . . . . . . . . . . . 95

9.2 Covariance Matrix $A^{-1} \ldots \ldots \ldots \ldots \ldots \ldots \ldots \ldots$

9.3 Pixel Temperature Covariance Matrix . . . . . . . . . . . . . . . 97

9.4 Correlation Functions of Correlated Noise . . . . . . . . . . . . . . 98

9.5 Auto-correlation Function Bias in Real Sky Maps . . . . . . . . . . . . 101

$10.1 \chi^{2}$ contours in $n, Q_{r m s-P S}$ space . . . . . . . . . . . . 109

10.2 Determinations of Power Spectrum Parameters . . . . . . . . . . . 110

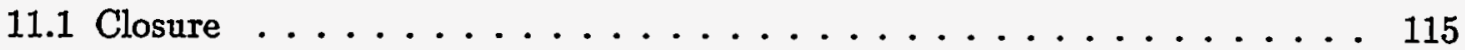

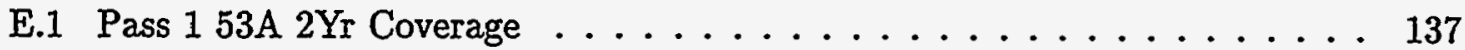

E.2 Pass 2 53A 2 Yr Coverage $\ldots \ldots \ldots \ldots \ldots \ldots \ldots \ldots \ldots \ldots \ldots \ldots \ldots \ldots$

E.3 Separate Channels for Yrl Pass 1 vs Pass $2 \ldots \ldots \ldots \ldots$ 


\section{List of Tables}

1.1 Anisotropy Mechanisms ....................... 13

2.1 DMR RMS Noise Values . . . . . . . . . . . . . . . . . . 24

5.1 Nested Velocities . . . . . . . . . . . . . . . . . 59

7.1 Frequency Dependence of Signal . . . . . . . . . . . . . 75

7.2 Statistical Significance as a Function of Frequency . . . . . . . . 76

7.3 RMS Temperature Fluctuations and $Q_{r m s}$ Estimates from $\sqrt{C(0)} \ldots \ldots 79$

8.1 Statistical Significance as a Function of Galactic Cut . . . . . . . . . 89

$10.1 n$ and $Q_{r m s-P S}$ fits to the Correlation Function . . . . . . . . . 105

10.2 Other $n$ and $Q_{r m s-P S}$ Determinations from DMR Data . . . . . . 108

11.1 Recent CMB Anisotropy Experiments . . . . . . . . . . . . 116

D.1 Diversity of Correlation Function Notation . . . . . . . . . . . . 134

E.1 Pass 1 versus Pass 2 Processing . . . . . . . . . . . . . . 136 


\section{Thesis Summary}

\section{Chapter 1 Cosmology and the Cosmic Microwave Background}

To provide a context for understanding the Differential Microwave Radiometer (DMR) measurements we discuss the standard hot big bang model, the cosmic microwave background (CMB) radiation and the surface of last scattering. We review the mechanisms of anisotropy generation concentrating on the large scales relevant to the DMR measurements. Finally, we discuss CMB anisotropy predictions based on the gravitational instability model of structure formation.

\section{Chapter 2 The DMR Experiment}

The purpose of the DMR instrument is to detect large angular scale anisotropies in the CMB. We describe the COBE orbit and how the DMR experiment is designed to meet this goal. We discuss Galactic foreground. We review the instrumentation, noise properties and how a DMR measurement is made.

\section{Chapter 3 Data Processing}

The main goal of the data processing is to turn the differential measurements into a sky map. We provide an overview of the software pipeline in which the data flow from the satellite to the maps. We present a description of the calibration, baseline removal and correction of the data which are then used to make the maps. We concentrate on describing how the map-making code operates.

\section{Chapter 4 Correlation Function: Theory versus Practice}

The two-point temperature correlation function provides an important characterization of CMB anisotropies. We present several simple examples to illustrate the use and power of a correlation function analysis. We describe its relation to the power spectrum and the role played by cosmic variance. We compare the theory to the nitty-gritty of the data analysis including incomplete sky coverage and the real DMR beam pattern. Additionally we describe a novel way to obtain the quadrupole amplitude of our observable Universe from the correlation function. 


\section{Chapter 5 The First Year Results}

We present a detailed correlation function analysis of the first year data set which contributed significantly to the DMR discovery of anisotropy and was partially reported in Smoot et al. (1992). We use the correlation function to help determine that the observed structure cannot be attributed to noise or Galactic foreground. As expected of a CMB signal, the correlation function signal is consistent with no frequency dependence and no Galactic latitude dependence. The dipole results from the first year maps are summarized and interpreted.

\section{Chapter 6 Correlation Function Error Analysis}

We compute error bars for $C(\alpha)$ from a standard propagation of errors formula and compare the results with a calculation of the covariance matrix of the correlation function with and without cosmic variance. Approximations for the error bar dependence on separation angle, Galactic plane cut and time are given.

\section{Chapter 7 Correlation Function Analysis of the Two Year Maps}

We present a detailed correlation function analysis of the two year (2YI) maps. The results support the conclusions based on the first year of data. The results of a novel method to obtain the rms quadrupole of our sky are given.

\section{Chapter 8 Correlation Function Comparison of the First and Second Year Maps} Confirmation of the DMR first year results can be obtained from a comparison with the independent second year DMR results. We present a correlation function comparison of the first year (Yr1) and second year (Yr2) data. The Yr1 and Yr2 data sets are reasonably consistent. The positive correlation for separation angles less than $\sim 20^{\circ}$ is robust to Galactic latitude cut and is very stable from year to year. Some of the differences between Yr1 and Yr2 are discussed.

\section{Chapter 9 Correlated Noise in the Maps}

The COBE DMR sky maps contain low-level correlated noise. We obtain estimates of the amplitude and pattern of the correlated noise from three techniques: angular averages of the covariance matrix, Monte Carlo simulations of two-point correlation functions, and direct analysis of the DMR maps. The results from the three methods are mutually consistent. The 
noise properties of the DMR maps are well approximated by the noise properties of maps made by a single beam experiment. Published COBE DMR results are not significantly affected by correlated noise.

Chapter 10 Obtaining $n$ and $Q_{r m s-P S}$ from the Correlation Function

We obtain the power law spectral index $n$ and the quadrupole normalization $Q_{r m s-P S}$ from the correlation functions of $\mathrm{Yr}_{\mathrm{r}}$, Yr2 and 2Yr DMR maps. We compare our results to other published $n$ and $Q_{r m s-P S}$ determinations.

\section{Chapter 11 Conclusions and the Future}

We summarize the thesis and draw useful conclusions about further analysis. We discuss the experimental assault on the power spectrum of CMB fluctuations.

\section{References}

\section{Appendix A Recent DMR Publications}

List of coauthored publications upon which some of the material in this thesis is based.

\section{Appendix B Thesis Notation}

A semi-exhaustive glossary of the notation used in this thesis.

\section{Appendix C DMR Notation}

A summary of the notation used in DMR publications.

\section{Appendix D Literature Notation}

A summary of the notation used in the literature to aid comparison with this thesis.

Appendix E Software Versions: Pass 1 vs Pass 2

A summary of the differences between Pass 1 and Pass 2 software.

Appendix F Friedmann Equation

Appendix G Antenna Temperature

A discussion of antenna and thermodynamic temperatures

Appendix $\mathrm{H}$ Spherical Harmonic Coefficients $a_{\ell m}$ and $b_{\ell m}$

A discussion of differences between the complex $a_{\ell m}$ and the real $b_{\ell m}$ spherical harmonic coefficients.

Appendix I Correlation Function Power Spectrum Expansion

Derivation of the power spectrum expansion of the correlation function. 
Appendix J Arbitrary Mean Value of Map Solution

Derivation of the arbitrariness of the mean of the DMR maps and why the iterative solution technique for inverting a singular matrix is valid.

Appendix K Errors on the Correlation Function

Appendix L Relation to Density Contrast Two-Point Correlation Function 


\title{
Chapter 1
}

\section{Cosmology and the Cosmic Microwave Background}

\begin{abstract}
To provide a context for understanding the Differential Microwave Radiometer (DMR) measurements we discuss the standard hot big bang model, the cosmic microwave background (CMB) radiation and the surface of last scattering. We review the mechanisms of anisotropy generation, concentrating on the large scales relevant to the DMR measurements. Finally, we discuss CMB anisotropy predictions based on the gravitational instability model of structure formation.
\end{abstract}

\subsection{The Standard Hot Big Bang Model}

Cosmology is a scientific attempt to answer fundamental questions of mythical proportion: How did the Universe come to be? How did it evolve? How will it end? Over the past century progress has been made towards answering these questions and has resulted in a standard hot big bang model describing the evolution of the Universe. This model provides a consistent framework into which all the relevant cosmological data seem to fit and is the dominant paradigm against which all new ideas are tested. In this Chapter we review the basic ideas of the standard big bang model. For a more complete description see the introductory chapters of Peebles (1993), Padmanabhan (1993), Kolb \& Turner (1990) and references therein. 
The big bang model of the Universe is based on the following observations.

\section{The Universe is expanding}

2. On the largest scales the Universe is isotropic and homogeneous

3. The Universe is filled with microwave photons coming from all directions

4. The Universe is composed of $\approx 75 \%$ hydrogen and $\approx 25 \%$ helium

Each observation and its implications are discussed separately below.

\section{The Universe is expanding}

In the early part of this century cosmology took a quantum leap when it was found that the nebulae are galaxies external to our Milky Way Galaxy and that curiously, they are all receding from us. This universal recession is interpreted as the expansion of the Universe and is codified in Hubble's law $v=H d$. That is, the recession velocity $v$ of a galaxy is proportional to its distance $d$ from us and $H$ is Hubble's constant. The implications of an expanding universe were profound. The Universe could no longer be considered static and it must have been smaller, denser and hotter in the past. A finite age for the Universe is postulated. Age determinations support this idea. So far no objects have been found with an age greater than 20 billion years. The dark night sky is also evidence for the the finite age of the observable Universe (Harrison 1987). A finite age however carries with it the notion of a creation event or a big bang. The origin of the Universe, $t=0$, seems to be the Achilles heel of the model. Is there a singularity at $t=0$ ? What happens before that? Strictly speaking however, the big bang origin of the Universe at $t=0$ is not part of the big bang model (Peebles 1993 p.6).

2. On the largest scales the Universe is isotropic and homogeneous The Universe looks the same in all directions and the matter seems to be smoothly distributed. Observations of the cosmic microwave background radiation, the $\mathrm{x}$-ray background and deep radio surveys provide solid evidence for the isotropy. Tests of homogeneity are more difficult since they require three-dimensional information. The largest galaxy red-shift surveys may be beginning to see homogeneity, however the assumption of homogeneity is based as much on mathematical convenience as observational evidence. Einstein's equations have a simple isotropic and homogeneous solution known as Friedmann's equation. It is the dynamical equation relating a universal scale factor $\mathrm{R}$ to the matter in the Universe (Appendix F). This allows us to write Hubble's law without reference 
to galaxies: $\dot{R}=H R$, where the dot indicates differentiation with respect to time and we take $R=1$ today. On small scales the Universe is not isotropic and homogeneous. There are lots of small scale structures in the Universe (galaxies, galactic clusters, voids, superclusters) and any model of the Universe needs to explain how they got there. Gravitational collapse of initially small density inhomogeneities is the standard big bang explanation. This is not very far-fetched since we are now falling at about $630 \mathrm{~km} / \mathrm{s}$ towards the largest local overdensity.

3. The Universe is filled with microwave photons coming from all directions This sea of photons is known as the cosmic microwave background radiation (CMB). The photon wavelengths are about as big as these letters and there are about 415 of them in every cubic centimeter of the Universe. This thesis is about measurements of these photons and their slightly anisotropic distribution.

4. The Universe is composed of $\approx 75 \%$ hydrogen and $\approx 25 \%$ helium

This is true for the visible baryonic matter i.e., stars, dust and gas. In the big bang model this three to one ratio was determined during an epoch of nucleosynthesis in the early Universe. Big bang nucleosynthesis (BBN) occurred within the first few minutes after the big bang. The agreement of $B B N$ predictions for the light nuclei abundances $\mathrm{H}, \mathrm{D},{ }^{3} \mathrm{He}$, ${ }^{4} \mathrm{He}$ and ${ }^{7} \mathrm{Li}$ provides the earliest solid evidence supporting the big bang model. The trace amounts of other elements were cooked up in stellar kitchens at much lower redshift. Since BBN occurred during the first three minutes after the big bang, it is often said that the big bang model has been tested that far back. In addition to this visible baryonic matter, there is much evidence that some kind of dark matter lurks about. The outlying parts of galaxies and galactic clusters are orbiting too fast to be contained by the visible matter.

In the 1980's, theorists devised an important extension of the standard big bang model called inflation. Inflation adds an early period of accelerating expansion to the history of the universe and also provides a mechanism (quantum fluctuations) for the production of the initially small density inhomogeneities needed for gravitational instability theories of structure formation. The accelerating expansion solves two initial condition problems of the standard big bang model:

(1) The horizon problem - the CMB has the same temperature in opposite directions yet the gas in those directions has never been in causal contact 
(2) The flatness problem - the density of the Universe is near the critical value of a flat universe yet the Friedmann equation tells us that the initial deviation from flatness would have had to be unbelieveably small for this to be the case today. Inflation solves these problems; it permits opposite sides of the observable universe to be in causal contact (i.e., in thermal equilibrium) before inflation and the expansion flattens any pre-inflationary curvature, yielding $\Omega=1$.

There are alternatives to the hot big bang model. See for example Layzer (1992) who advocates a cold big bang model. A critique of the hot big bang model is presented in Arp et al. (1990) while orthodoxy is well-defended and compared with other alternatives in Peebles et al. (1991).

\subsection{CMB and the Surface of Last Scattering}

The observable universe is expanding and cooling. Therefore in the past it was hotter and smaller. The cosmic microwave background (CMB) radiation is the after glow of thermal radiation left over from this hot early epoch in the evolution of the Universe. The CMB is a bath of photons coming from every direction. These are the oldest photons one can observe and they contain information about the Universe at redshifts much larger than the redshifts of galaxies or quasars. The CMB is thus a unique tool for probing the early Universe.

The prediction of the existence and the temperature of a CMB in 1948 (Alpher \& Herman 1948) followed by its detection in 1964 (Penzias \& Wilson 1965, Dicke et al. 1965) provides possibly the strongest evidence for the big bang. The CMB detection began the search for the determination of its exact spectrum and level of anisotropy. A CMB of truly cosmic origin is expected to have a blackbody spectrum and to be extremely isotropic.

COBE observations show that the CMB is very well approximated by an isotropic blackbody. The recently published COBE FIRAS result is that the CMB has the spectrum of a blackbody at a temperature of $T_{0}=2.726 \pm 0.01 \mathrm{~K}$ (95\%CL) (Mather et al. 1994). Figure 1.1 shows how well the FIRAS measurements along with many other measurements agree with a blackbody spectrum at $T_{0}=2.726 \mathrm{~K}$. Figure 1.2 displays the DMR $53 \mathrm{GHz}$ two year map. Not only is the CMB blackbody, it is also highly isotropic. 


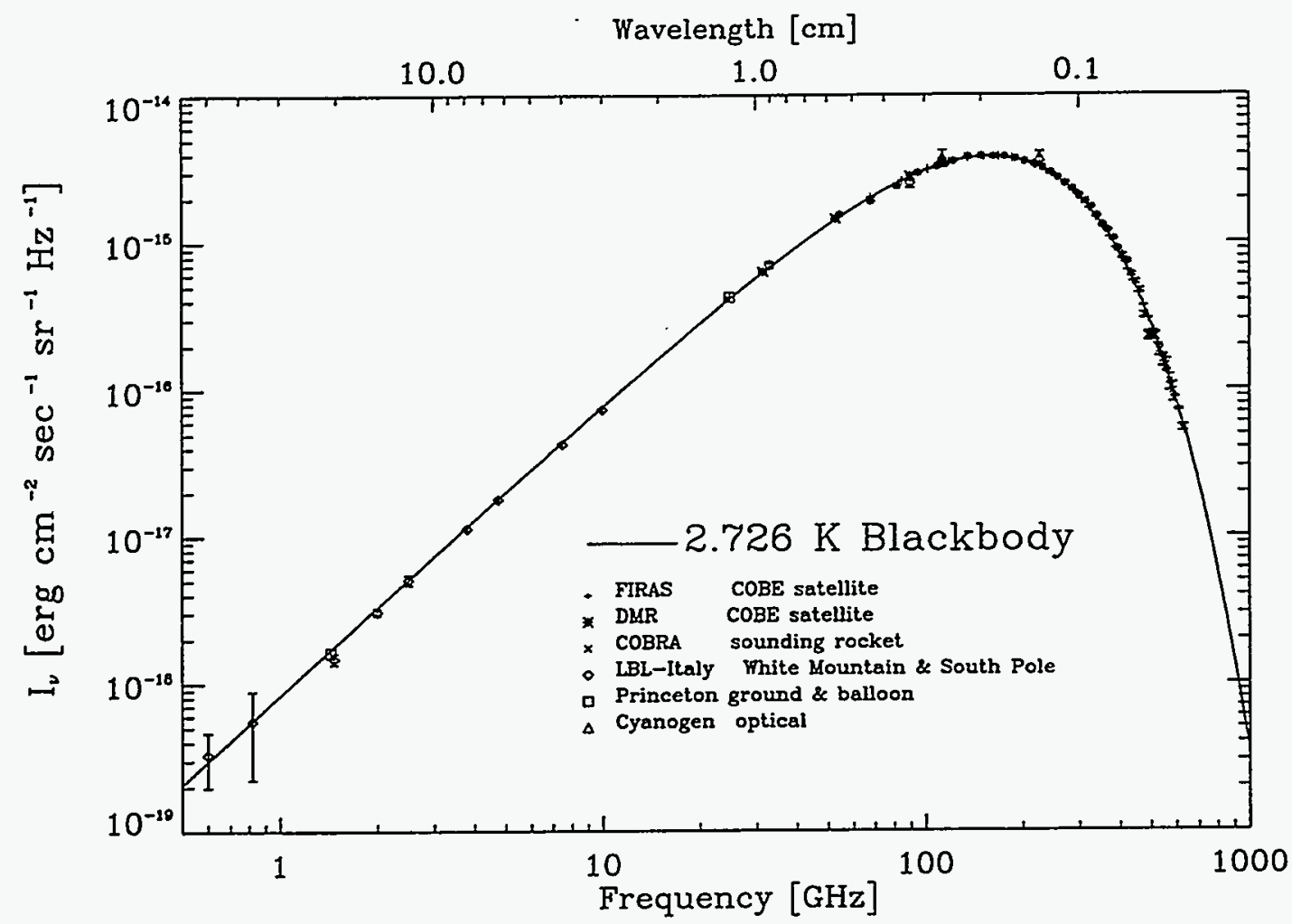

Figure 1.1: CMB Spectrum Measurements. The spectrum of the CMB has been measured over 3 decades in frequency and found to be consistent with a blackbody at $T_{0}=2.726 \mathrm{~K}$.
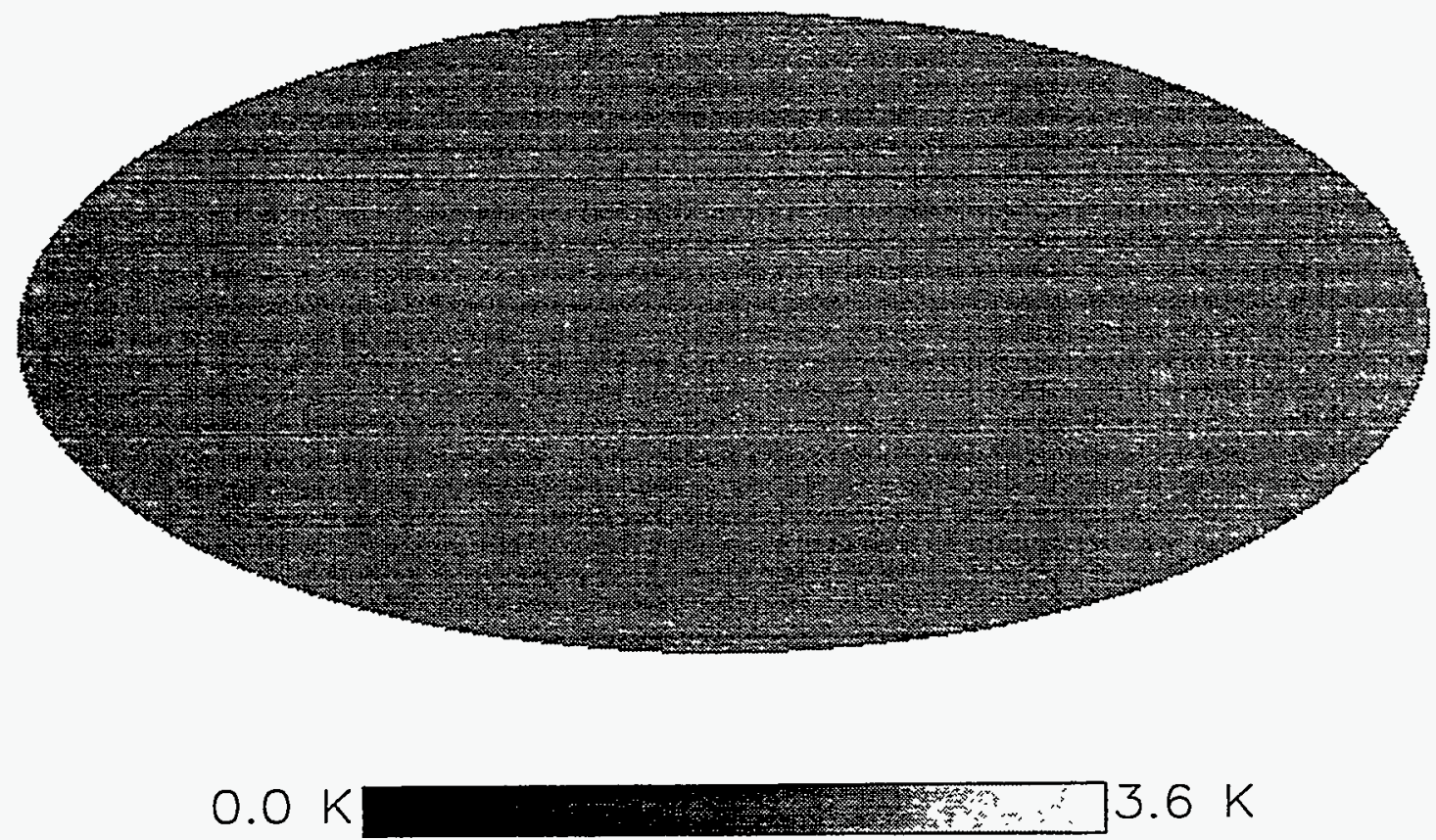

Figure 1.2: CMB isotropy. DMR two-year $53 \mathrm{GHz}$ full-sky map in galactic coordinates. The CMB is very well approximated by a perfectly isotropic blackbody. 


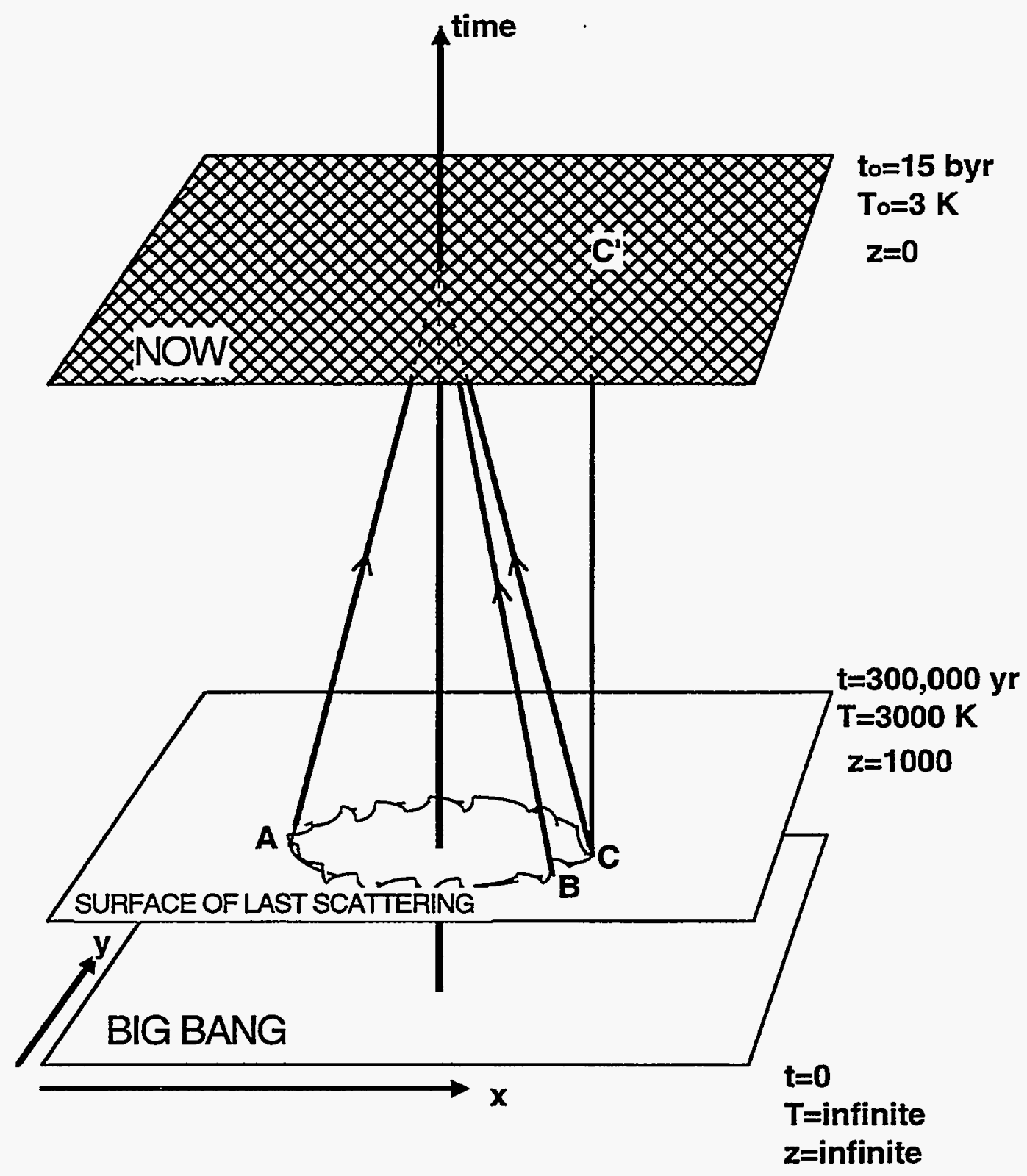

Figure 1.3: Space-time and the surface of last scattering. The time axis is the world line of the stationary observer who is currently located at the apex of the light cone. CMB photons travel from the wavy circle in the surface of last scattering along the surface of the light cone to the observer. Points $\mathrm{A}$ and $\mathrm{C}$ are in opposite sides of the sky. If the angle between $\mathrm{B}$ and $\mathrm{C}$ is greater than a few degrees then $\mathrm{B}$ and $\mathrm{C}$ have not been in (post-inflational) causal contact. The unevenness of the circle represents potential fluctuations at the surface of last scattering. The bottom two planes are at fixed times while the "NOW" plane moves upward. As it does, the size of the visible universe (wavy circle) increases. The object seen at $\mathrm{C}$ is currently at $\mathrm{C}^{\prime}$. 
At approximately $10^{-2}$ seconds after the big bang the universe was about $10^{10} \mathrm{~K}$ and nucleosynthesis began. At about 300,000 years after the bang, the universe had cooled down enough to allow the free electrons and protons to combine to form neutral hydrogen. This period is known as recombination or decoupling. This neutralization of the plasma allowed photons to free stream in all directions.

Before recombination the universe was an opaque fog of free electrons, afterwards it was transparent. The boundary is called the cosmic photosphere or the surface of last scattering. As its name implies, the surface of last scattering is where the CMB photons were Thomson scattered for the last time before arriving in our detectors (Figure 1.3). Except for the tiny $\left(10^{-9}\right)$ contribution of one Lyman- $\alpha$ photon per hydrogen atom, the CMB photons were not produced at this time, they were only scattered.

The surface of last scattering can be described by several parameters. Here we derive the redshift $z_{\ell s}$, the temperature $T_{\ell_{s}}$ and the time $t_{\ell_{s}}$ of last scattering. As the Universe expands it cools. The CMB photons get redshifted and their blackbody temperature goes down. The fact that a redshifted blackbody remains a blackbody can be shown using the Lorentz invariance of $I_{\nu} / \nu^{3}$ or equivalently the mean photon occupation number. Since the expansion redshift $z$ is defined by

$$
1+z=\frac{\lambda_{o}}{\lambda_{e}}=\frac{1}{R\left(t_{e}\right)}
$$

(where the $e$ and $o$ stand for emitted and observed respectively), and since the temperature scales as $T \propto 1 / R \propto 1+z$, the temperature as a function of redshift is

$$
T(z)=T_{o}(1+z)
$$

Recombination occurs when the CMB temperature has dropped to the point when there are no longer enough high energy photons in the CMB to keep hydrogen ionized; $\gamma+H \leftrightarrow e^{-}+p$. Although the ionization potential of hydrogen is $13.6 \mathrm{eV}\left(T \sim 10^{5} \mathrm{~K}\right)$ recombination occurs at $T \approx 3000 \mathrm{~K}$. The high photon to proton ratio $\left(\eta \approx 10^{9}\right)$ allows the high energy tail of the Planck distribution to keep the comparatively small number hydrogen atoms ionized until this much lower temperature. The Saha equation (e.g. Lang 1980) describes this balance between the ionizing photons and the ionized and neutral hydrogen. As the temperature decreases, an increasing Boltzmann factor suppresses ionization while the large photon to proton ratio, $\eta$, maintains it. Recombination occurs when we have

$$
e^{-\frac{\chi}{k T}} \sim \eta
$$


where $\chi$ is the ionization potential. The large value of $\eta$ allows $\mathrm{T}$ to get as low as $8000 \mathrm{~K}$. In addition, trapped Lyman- $\alpha$ photons keep much of the neutral hydrogen in an excited state making it easier to ionize (see e.g. Silk 1989). The result is that recombination occurs at $T_{\ell s} \approx 3000 \mathrm{~K}$. Equation (1.2) then yields $z_{\ell s} \approx 1100$.

We get the time of last scattering using the time dependence of the scale factor $R$ in the matter dominated regime. Inserting $\rho \propto R^{-3}$ in the Friedmann equation (see Appendix F) yields $R(t) \propto t^{2 / 3}$. Thus,

$$
\frac{T_{\ell s}}{T_{0}} \approx \frac{3000}{2.7} \approx\left(\frac{t_{o}}{t_{\ell s}}\right)^{2 / 3}
$$

Therefore if the present age of the universe is $t_{0} \sim 10 \times 10^{9}$ years, then $t_{\ell s} \sim 3 \times 10^{5}$ years after the big bang. Thus the CMB photons have come to us from the surface of last scattering which can be described by the temperature, redshift and time

$$
\begin{aligned}
T_{\ell s} & \approx 3000 K \\
z_{\ell s} & \approx 1100 \\
t_{\ell s} & \approx 3 \times 10^{5} \text { years. }
\end{aligned}
$$

The surface of last scattering is at a fixed temperature and time after the big bang: $t_{\ell s}=$ constant and $T_{\ell s}=$ constant. Thus from equation (1.2) we find that the surface of last scattering is receding from us with an ever-increasing redshift, $z_{\ell s} \propto \frac{1}{T_{0}(t)}$.

The size of a causally connected region on the surface of last scattering is important because it determines the size over which astrophysical processes can occur. A causally connected Hubble patch at last scattering is $L_{H}=3 c t_{\ell s}\left(1+z_{\ell s}\right) \sim 200 h^{-1} M p c$ which subtends an angular size $\theta_{H}$

$$
\theta_{H} \approx 1^{\circ} \Omega_{\circ}^{1 / 2}\left(\frac{z_{\ell s}}{1000}\right)^{-1 / 2}
$$

Since the DMR beam averages over patches approximately $7^{\circ}$ across, the smallest spots detected by the DMR at the surface of last scattering are well into the causally disconnected $\theta>\theta_{H}$ regime.

The thickness of the surface of last scattering is $\Delta z \approx 80$ which corresponds to a length $\Delta L \approx 7 \Omega^{-1 / 2} h^{-1} \mathrm{Mpc}$ or an angular size of $\Delta \theta \approx 8^{\prime} \Omega^{-1 / 2}$ (Kaiser \& Silk 1986). Anisotropies on scales smaller than about $8^{\prime}$ are suppressed because they are superimposed on each other over the finite path length of the photon in the surface. It is possible that high redshift sources of ultraviolet photons reionized the hydrogen or kept it from recombining. This 
reionization increases the effective thickness of the surface of last scattering and suppresses anisotropies on scales larger than $8^{\prime}$. For example for reionization $z_{\text {reion }}>200$, anisotropies at scales less than $\sim 1^{\circ}$ are suppressed while for $z_{\text {reion }}>20$, anisotropies at scales less than $\sim 5^{\circ}$ are suppressed (Bartlett and Stebbins 1992, Bond 1993). Notice however that the DMR results (scales $>7^{\circ}$ ) are uneffected by this reionization suppression.

\subsection{Anisotropy Mechanisms in a Perturbed Robertson- Walker Universe}

To a very good approximation the CMB is a flat featureless blackbody; there are no anisotropies and the temperature is a constant $T_{o}=2.726 \mathrm{~K}$ in every direction. This is seen in Figure 1.4 (top). When we remove the mean, the next largest feature visible at 1000 times smaller amplitude is the kinetic dipole displayed in the middle panel of Figure 1.4.

Anisotropies in the CMB are expected due to a variety of effects. The physical causes of the anisotropy can be conveniently attributed to pre-recombination, recombination and post-recombination epochs (Bertschinger, 1993). Pre-recombination anisotropies are due to photon "fluid compression", gravitational time delay, or entropy fluctuations. Recombination anisotropies are due to gravitational and Doppler red/blueshifts and post-recombination anisotropies are from differential gravitational redshifts in evolving potentials. Pre-recombination and recombination anisotropies are sometimes collectively referred to as primary anisotropies while post-recombination anisotropies are also known as secondary anisotropies.

The differential temperature of the CMB in direction $\vec{n}, \delta T=T(\vec{n})-T_{o}$, can be expressed as

$$
\frac{\delta T(\vec{n})}{T_{o}}=\frac{\vec{n} \cdot \vec{V}_{\odot}}{c}-\frac{\vec{n} \cdot \vec{V}_{\ell s}(\vec{n})}{c}+\frac{\Phi_{\ell s}(\vec{n})}{3 c^{2}}+\frac{2}{c^{2}} \int \frac{\partial}{\partial t} \Phi(t, \vec{n}) d t,
$$

where $\vec{V}_{\odot}$ is the Sun's velocity w.r.t. the CMB and $\vec{V}_{\ell s}(\vec{n})$ is the velocity of the surface of last scattering w.r.t. the rest frame of the CMB. The potential fluctuations $\Phi$ are related to the mass density by the Poisson equation $\frac{1}{R^{2}} \nabla^{2} \Phi=4 \pi G \delta \rho$. The first two terms in equation (1.9) are Doppler effects. The first produces the Great Cosine on the sky seen in the middle panel of Figure 1.4 (see also section 5.5). The second term causes sub-horizon fluctuations responsible for the Doppler peak in the power spectrum on degree scales. 
The $7^{\circ}$ DMR beam averages over many of these fluctuations and is not sensitive to this second term. The third and fourth terms are sometimes referred to as the Sachs-Wolfe and the integrated Sachs-Wolfe terms respectively (Sachs \& Wolfe 1967).

Consider the third term of equation (1.9), $\Phi / 3 c^{2}$, due to the gravitational redshift of a time independent potential difference. The Pound-Rebka experiment used the Mossbauer effect to confirm the existence of a gravitational redshift of magnitude $\Phi / c^{2}$. So where did the $1 / 3$ come from in the third term? Assuming adiabatic fluctuations the StephanBoltzmann law yields $\frac{\delta T}{T_{0}}=\frac{1}{4} \delta \rho_{r} / \rho_{r}$, where $\rho_{r}$ is the radiation energy density. On superhorizon scales $\delta \rho_{r} / \rho=-\frac{8}{3} \Phi / c^{2}$ (Stebbins 1993). Thus super-horizon adiabatic fluctuations produce $\delta T / T_{0}=-\frac{2}{3} \Phi / c^{2}$, which cancels $2 / 3$ of the gravitational redshift result leaving $\Phi / 3 c^{2}$. Therefore although deep potential wells produce large gravitational redshifts, the photons coming out of them are not as redshifted as one would naively expect.

The time-dependent Sachs-Wolfe effect, or Rees-Sciama effect, is caused by the timedependent potentials of collapsing over-densities (producing redshifts) and expanding voids (producing blueshifts). These anisotropies are on very small scales and like the Doppler anisotropies on the surface of last scattering, are smoothed over by the DMR beam. Notice that all four terms in equation (1.9) are independent of the frequency of the radiation. This spectral flatness is used by observers to distinguish $\mathrm{CMB}$ anisotropies from Galactic and extragalactic foregrounds.

Figure 1.4 (bottom) is the smoothed full-sky two-year $53 \mathrm{GHz} A+B$ map. Some of the more prominent light and dark blurry spots above and below the horizontal plane of the galaxy are the largest and oldest structures ever detected. The spots are anywhere from a few degrees to a few tens of degrees across; too big to be causally connected at decoupling without invoking inflation. At these angular scales, the gravitational red- and blueshifts of the Sachs-Wolfe effect are believed to be the cause of the hot and cold spots,

$$
\frac{\delta T}{T_{o}}(\vec{n})=\Phi_{\ell s}(\vec{n}) / 3 c^{2}
$$

Since $\Phi_{\text {underdensity }}>\Phi_{\text {overdensity }}$ we have

$$
T\left(\vec{n}_{\text {underdensity }}\right)>T\left(\vec{n}_{\text {overdensity }}\right)
$$

Thus hot spots are mass underdensities and the cold spots are mass overdensities. By today these hot and cold spots, which we are seeing as they were about 15 billion years ago, have become the largest voids and clusters of superclusters of galaxies that anyone has 


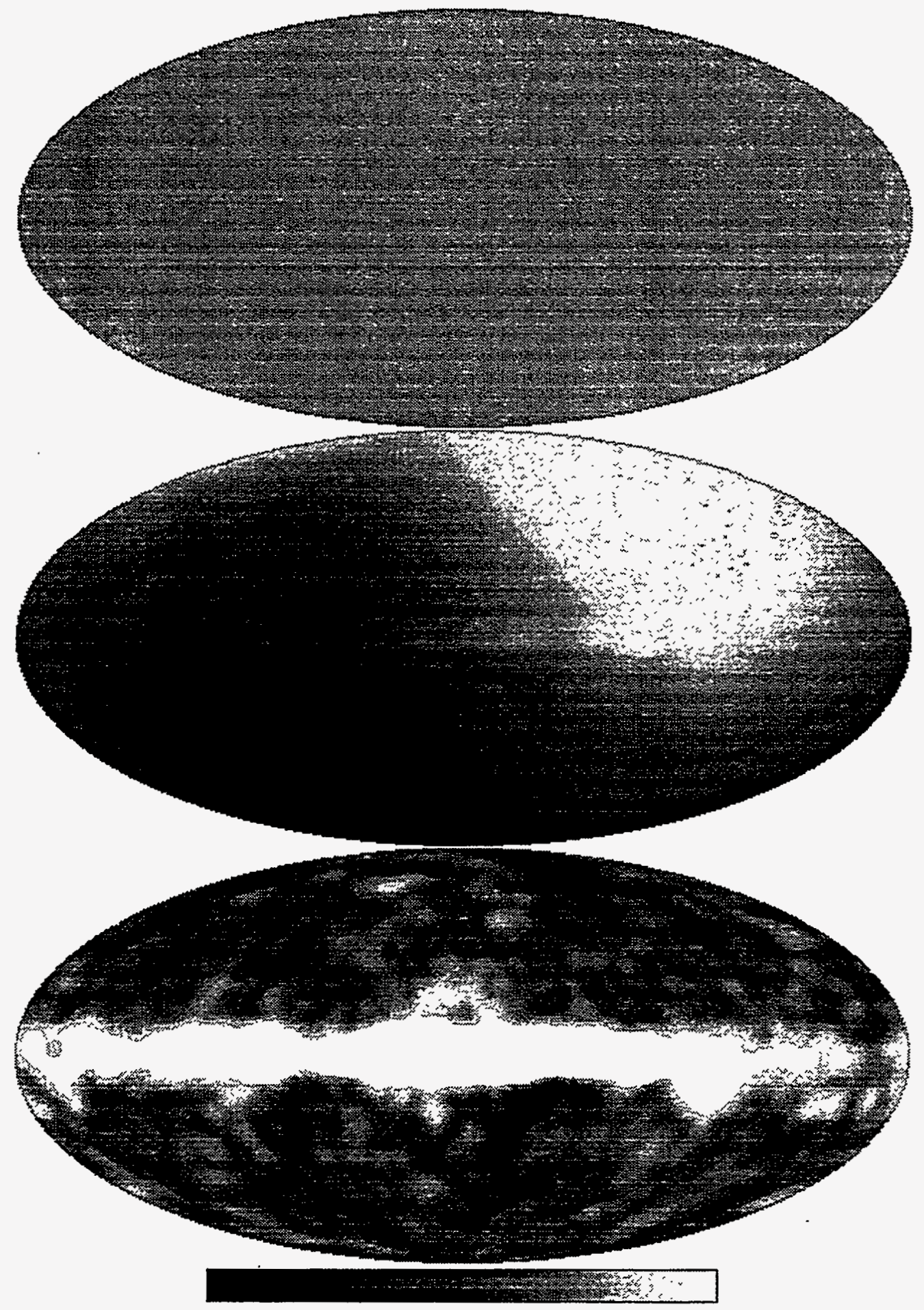

Figure 1.4: Monopole, Dipole and Structure with Noise (top, middle and bottom) from the $53 \mathrm{GHz}$ smoothed two year maps. From top to bottom the greyscale limits are $[0,3.6 \mathrm{~K}]$, $[-3.4,3.4 \mathrm{mK}]$ and $[-150,150 \mu \mathrm{K}]$. 
ever detected. The relevant equation giving the size in units of $100 \mathrm{Mpc}$ and justifying this claim is

$$
\text { size today } \approx \frac{\theta}{1^{\circ}}\left(\Omega_{0} h\right)^{-1} 100 \mathrm{Mpc}
$$

where $\theta$ is the angular size of the structure in the maps. Plugging in a typical $\theta$ of $20^{\circ}$ one obtains $2000\left(\Omega_{0} h\right)^{-1} \mathrm{Mpc}$. Recall that $0.5 \lesssim h \lesssim 1$, inflation predicts $\Omega_{0}=1$ and that the size of the observable universe is $\approx 3000 \mathrm{Mpc}$.

For simplicity, in equation (1.9), we have assumed a Robertson-Walker metric and therefore do not consider differential expansion as a source of anisotropy. Additionally, we do not include the frequency dependent Vishniac (1987) and Sunyaev-Zel'dovich (1972) effects. These are post-recombination effects which contribute to small angular scale anisotropy to which the DMR is insensitive. We also do not include the more speculative anisotropies due to topological defects (monopoles, strings, walls, textures) or any contribution from a possible rotation of the Universe (Barrow, Juszkiewicz \& Sonoda 1985). We also do not include polarization anisotropies. Table 1.1 summarizes this larger array of CMB anisotropy mechanisms.

\subsection{Structure Formation and Anisotropy Predictions}

The galaxies around us are clustered on scales from $1 \mathrm{Mpc}$ (our Local Group) up to $\sim 100 \mathrm{Mpc}$ (great walls, sheets and voids). If these structures formed from overdensities which gravitationally collapsed, the overdensities must have been present at decoupling and must have produced temperature anisotropies on the surface of last scattering. Observers have been searching for the expected small temperature anisotropies ever since the discovery of the CMB. Predictions for the level of CMB anisotropy have decreased to keep pace with the observations: "We have estimated that anisotropies of order 1 per cent should occur in the microwave radiation" (Sachs \& Wolfe 1967) and a year later, "...that part of the temperature anisotropy in the microwave radiation at the present epoch which is due to primordial anisotropies should be less than 0.03 per cent (of $3 \mathrm{~K}$ )." (Misner 1968).

Calculating the expected $\Delta T / T_{o}$ level from large scale structure $\delta \rho / \rho$ levels without invoking dark matter leads one to the conclusion that these overdensities (and underdensities) should have been discovered 15 years ago. As experimenters found the surface of last scattering to be smoother and smoother, dark matter was invoked to keep the predicted level of temperature variations below experimental limits. Since dark matter 
structures were able to start growing when the Universe became matter dominated at $z_{e q} \sim 10^{4}$, evoking dark matter reduces the predicted value of $\delta T / T_{0}$ by about a factor of $z_{\ell s} / z_{e q} \sim 1 / 10$. The DMR instrument aboard the COBE satellite was prudently constructed in the pre-dark matter epoch with enough sensitivity to probe the post-dark matter prediction levels for the expected anisotropies.

Table 1.1: Anisotropy Mechanisms

\begin{tabular}{|c|c|c|c|c|}
\hline Name & Angular Scale & Source & redshift & $\delta T / T \propto$ \\
\hline Doppler(Sun) & large & velocity of Sun & 0 & $\propto V_{\odot} / c$ \\
\hline Doppler $(\ell s)$ & degree & velocity of surface $\ell_{\ell s}^{a}$ & $z_{\ell s}$ & $\propto V_{\ell s} / c$ \\
\hline $\mathrm{SW}^{b}$ & large & potential difference & $\gtrsim z_{\ell s}$ & $\Phi / 3 c^{2}$ \\
\hline Integrated SW & small & time dependent potentials & $<z_{\ell s}$ & $\frac{2}{c^{2}} \int \frac{\partial \Phi}{\partial t} d l$ \\
\hline Vishniac $^{b}$ & $<$ degree & angular momentum & $<z_{\ell s}$ & \\
\hline $\mathrm{SZ}^{c}$ & $\sim \operatorname{arcmin}$ & hot electrons & $<z_{\ell s}$ & $\int \frac{k T_{e}}{m_{e} c^{2}} d l$ \\
\hline Topological Defects & & phase transitions & any $z$ & $\propto{\frac{G \mu}{c^{2}}}^{d},{\frac{G \sigma}{H_{0}}}^{e}$ \\
\hline Rotation of Universe & large & vorticity & $>z_{\ell s}$ & \\
\hline
\end{tabular}




\title{
Chapter 2
}

\section{The DMR Experiment}

\begin{abstract}
The purpose of the DMR experiment is to detect large angular scale anisotropies in the CMB. We describe the COBE orbit and how the DMR instrument is designed to meet this goal. We discuss the Galactic foregrounds. We review the instrumentation, noise properties and how a DMR measurement is made.
\end{abstract}

\subsection{COBE Mission}

COBE is NASA's first cosmology satellite and was launched successfully Nov 18, 1989 on a Delta rocket. The primary goals of the COBE satellite are to measure the CMB spectrum and anisotropy, and measure the diffuse infrared background from primordial objects forming in the early universe. The three instruments designed to achieve these goals are the Far-Infrared Absolute Spectrophotometer (FIRAS), the Differential Microwave Radiometer (DMR) and the Diffuse Infrared Background Experiment (DIRBE). Secondary goals of the mission include measuring radiation from our local environment: interplanetary dust, interstellar electrons, starlight and other Galactic emission. These local sources can mask and limit the accuracy of the cosmological measurements.

Soon after launch FIRAS was able to report that the CMB was very nearly a perfect blackbody. The most recent FIRAS result is $T_{0}=2.726 \pm 0.01 \mathrm{~K}$ (95\% CL) (Mather et al. 1994). The DMR has detected CMB anisotropies and the difficult job of detecting an infrared background behind the ecliptic and galactic dust foregrounds is still going on with the DIRBE data. 

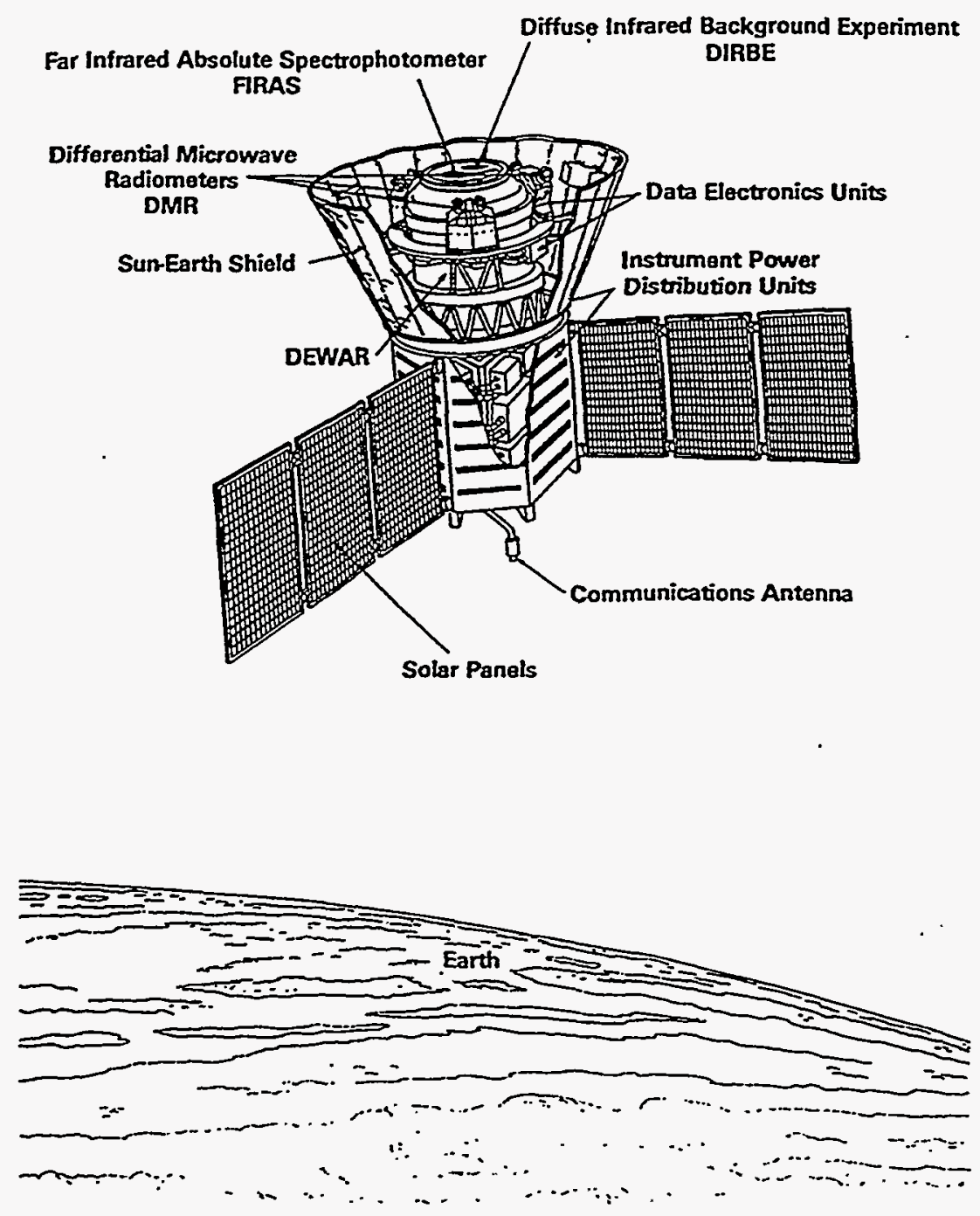

Figure 2.1: COBE satellite in orbit. COBE is about the size of a large van and weighs $2270 \mathrm{~kg}$ (Boggess et al. 1992). All three instruments are shielded from the Sun and Earth by a conical shield providing thermal stability. The Sun-Earth shield has been partially cut away to display the three DMR instrument modules mounted to the periphery of the liquid helium dewar. Looking down on the instruments, the spacecraft rotates counter-clockwise. The solar panels provide 750 Watts of power of which the DMR uses about $15 \%$. The low gain antenna visible at the bottom is used to communicate with the TDRSS satellite system. Viewed from the COBE orbit, the Earth subtends $\pi$ steradians or $1 / 4$ of the full sky. 


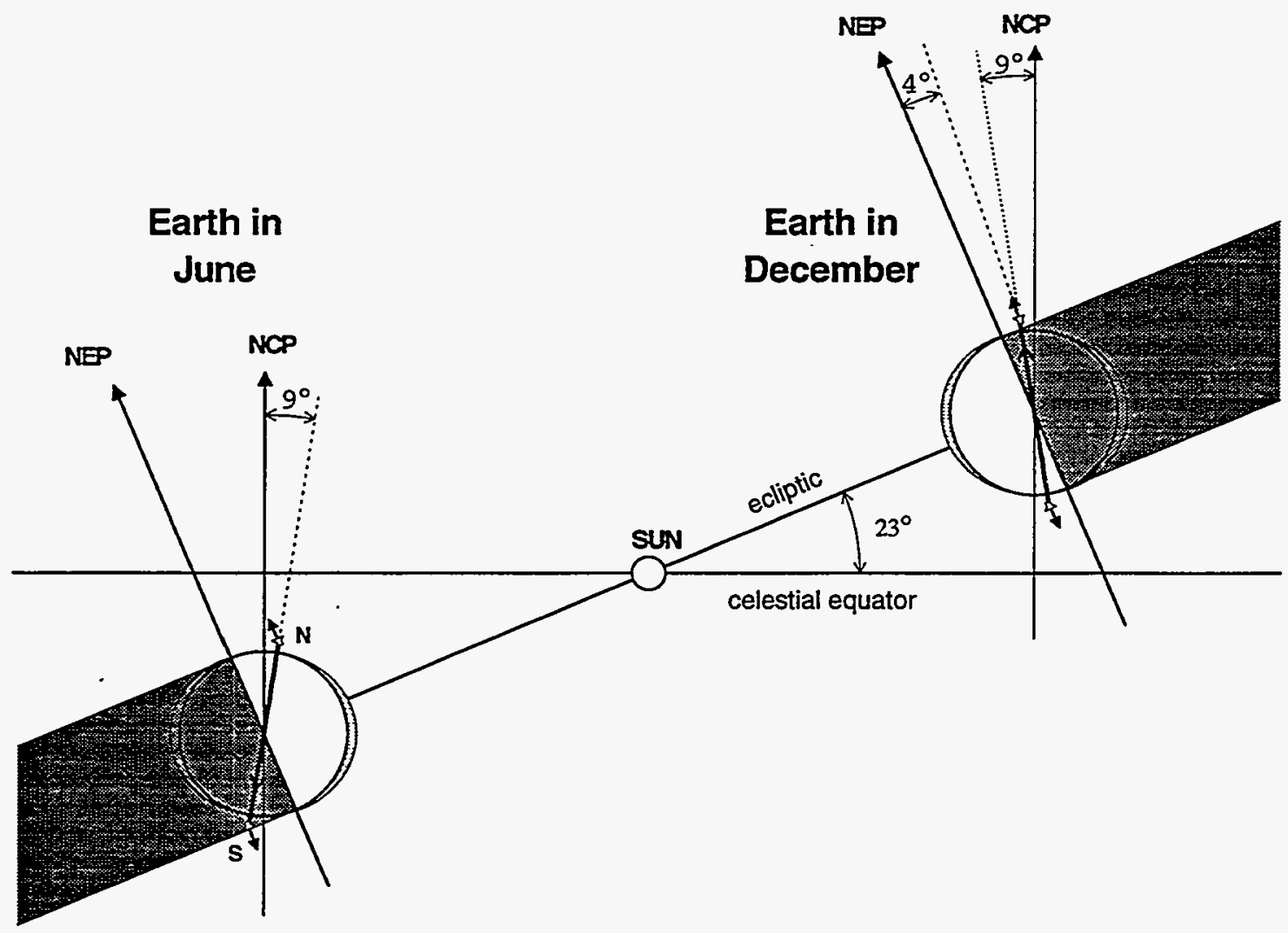

Figure 2.2: COBE's Orbit. The Earth is shown at both the winter and summer solstices. COBE's orbit is the thick line inclined $9^{\circ}$ from the north celestial pole (NCP). The COBE satellite is represented by the small white icon on the most northern and southern points of the orbit. The short arrow attached to COBE indicates the spin axis. During the eclipse season ( 2 months centered around the summer solstice) the satellite enters the Earth's shadow once per orbit in the south ('S'). In the north (' $N$ '), keeping the Sun-spin axis angle of $92^{\circ}$ allows the limb of the Earth to appear a few degrees above the shield in the anti-solar direction. The arrow on the orbit indicates the direction of COBE's $7.4 \mathrm{~km} / \mathrm{s}$ velocity. The satellite was launched south at dawn. Every orbit, the spin axis passes within $2^{\circ}$ to $4^{\circ}$ of the north ecliptic pole (NEP). Figure 2.3 displays the resultant sky coverage pattern. 


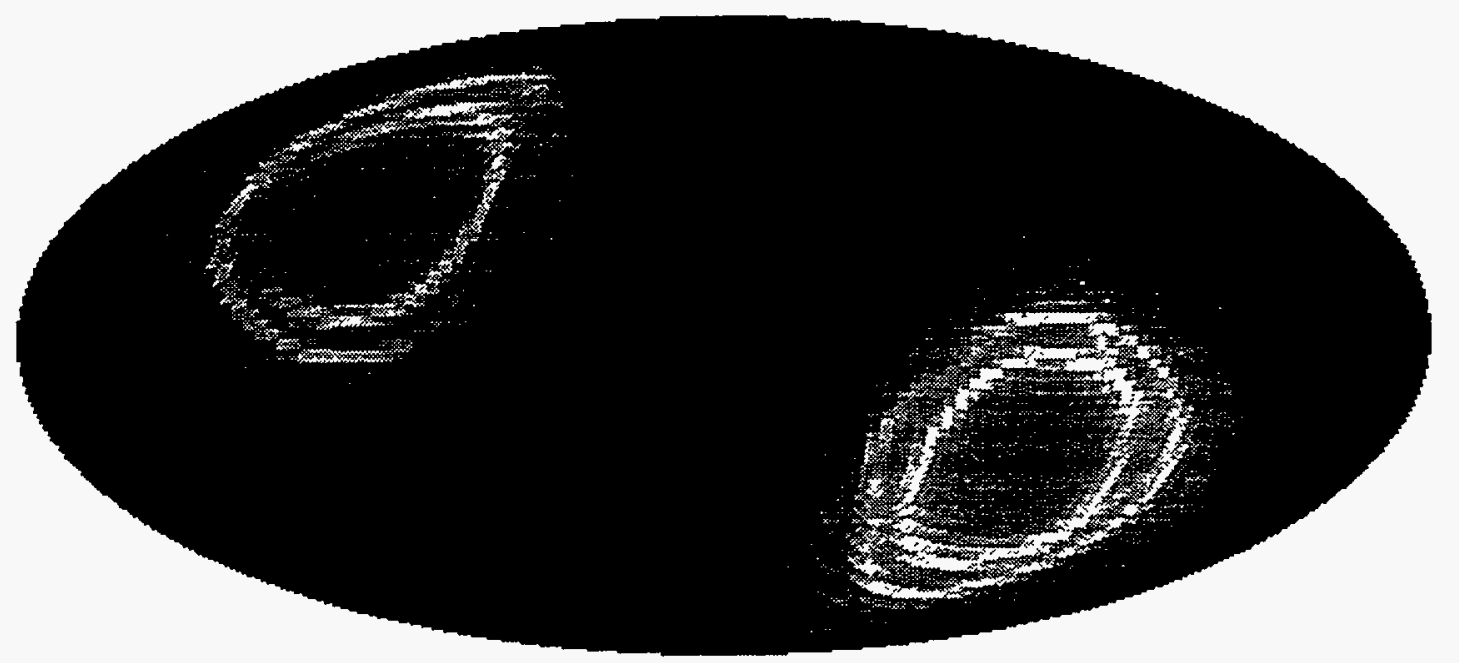

21526 81271

Figure 2.3: Full sky map in Galactic coordinates of the number of observations per pixel for the two year 53A map. The regions of maximum observation are two $60^{\circ}$ diameter rings centered on the north and south ecliptic poles. The minimum observation regions near the ecliptic equator are caused by the scanning strategy and the Moon cuts. The cuts required by the Earth limb appearing over the shield during the the eclipse season are evident in low values in the otherwise heavily sampled northern ring. Each maximum ring is split into two concentric rings because the direction of the spacecraft spin axis does not pass over the ecliptic poles but passes $2^{\circ}$ to $4^{\circ}$ from it (Figure 2.2). Notice that the maximum and minimum differ by a factor of four. There are 6144 pixels in the map. 


\subsection{COBE Orbit and DMR Observing Strategy}

COBE is in a $900 \mathrm{~km}$ altitude, near-terminator orbit with a 103 minute period. The combination of the $99^{\circ}$ inclination of the orbit, $900 \mathrm{~km}$ altitude and the quadrupole moment of the Earth results in a torque on the plane of the orbit causing it to precess $\approx 1^{\circ}$ per day. This precession rate keeps the orbital plane as close to perpendicular as possible to the Earth-Sun line. The relative positions of the Earth, Sun and orbital plane during the winter and summer solstices are displayed in Figure 2.2. The satellite spins with a 73 second period (0.815 rpm). The spin axis points away from the Earth and $92^{\circ}-94^{\circ}$ away from the Sun. This spinning, orbiting and precessing, combined with the fairly large $7^{\circ} \mathrm{FWHM}$ beams, enables the $\mathrm{DMR}$ to sample the entire sky in $\approx 5$ months. Figure 2.3 is a map of the sky coverage (number of observations per pixel) after two years of observation.

There are two channels at each frequency denoted 31A, 31B, 53A, 53B, 90A and 90B. Each channel (with the exception of $31 \mathrm{~A}$ and $31 \mathrm{~B}$ which share a pair of horns) has two corrugated aluminum horns. Their pointing directions are $60^{\circ}$ away from each other and $30^{\circ}$ away from the satellite's spin axis. Every half second the DMR measures the difference between the power entering the two horns, hence these are differential rather than absolute measurements.

The horn separation of $60^{\circ}$ was chosen as a compromise. A small opening angle minimizes problems with the Earth limb and Sun in the sidelobes however each pixel is differenced with only a small number of nearby pixels and complete sky coverage takes

longer. Increasing the number of pixel-pairs enhances the ability of the map-making algorithm to find a stable solution. The maximum number of pixel pairs occurs at a horn separation of $90^{\circ}$ but a $90^{\circ}$ separation would cause more sidelobe difficulties.

\subsection{Galactic Foreground}

The DMR has several advantages over ground and balloon based measurements: it can see the full sky with no atmospheric foreground and with very little Earth emission in the sidelobes. Galactic foreground however does not go away in orbit. Galactic emission from synchrotron radiation, bremsstrahlung and interstellar dust are the most problematic foregrounds for the DMR. The three DMR frequencies, $31.5,53$ and $90 \mathrm{GHz}$, were chosen to be near the peak of the $2.7 \mathrm{~K}$ Planckian CMB spectrum, to occupy the region between the higher frequency dust foreground and the lower frequency bremsstrahlung and synchrotron 
emission and to be in officially protected frequency bands (Smoot et al.1990, Bennett et al. 1992b).

At low frequencies synchrotron emission dominates. Synchrotron radiation is emitted by relativistic electrons spiraling in the $\sim 1 \mu \mathrm{G}$ magnetic fields of the Galaxy. The synchrotron antenna temperature signal is

$$
T_{\text {sync }}(\nu) \propto \nu^{\beta_{s y n c}} .
$$

The exponent $\beta_{\text {sync }} \approx-2.8$ and is $\nu$ dependent at DMR frequencies varying in the range -2.75 to -3.2 . At frequencies near $31 \mathrm{GHz}$ the bremsstrahlung emission is at approximately the same level as the synchrotron emission. Bremsstrahlung is emitted by non-relativistic free electrons as their paths are deflected by the E-fields of protons (e.g. Longair 1992). The bremsstrahlung antenna temperature signal is

$$
T_{f f}(\nu) \propto \nu^{\beta_{f f}}
$$

The exponent $\beta_{f f} \approx-2.1$ and is very weakly $\nu$ dependent at DMR frequencies varying in the range -2.07 to -2.15 . The HII regions around groups of $O$ and $B$ stars in the arms of our Galaxy are strong emitters of bremsstrahlung. The hot regions in the Galactic plane visible in the Cygnus $\left(\ell \sim 80^{\circ}\right)$ and Vela regions $\left(\ell \sim-80^{\circ}\right)$ are due to the superposition of several O-B associations. Cygnus is a superposition of 5 large HII regions and is about $7^{\circ}$ across. At higher frequencies, cold interstellar dust $(T \sim 20 K)$ becomes the dominant foreground,

$$
T_{d u s t}(\nu) \propto \nu^{\beta_{\text {dust }}},
$$

where $\beta_{\text {dust }} \approx 1.5$.

Figure 2.4 summarizes the frequency dependence of these three types of Galactic foreground. Notice how the DMR frequencies span the minimum. The grey bands reflect the degree of variation in the signal in the Galactic latitude range $15^{\circ} \lesssim|b| \lesssim 60^{\circ}$. The Galactic longitude dependence of the signals can not be represented in such a diagram. The width of the bands in Figure 2.4 indicate which foreground is most confined to the Galactic plane. The thin synchrotron band for example shows that relativistic electrons are less confined to the Galactic plane than are the non-relativistic electrons in HII regions. The slight frequency dependence of the exponents, $\beta_{s y n c}, \beta_{f f}, \beta_{\text {dust }}$ cause the valley of minimum emission at $\sim 60 \mathrm{GHz}$ to be slightly lower than a linear extrapolation would produce. Bennett et al. (1992b) describe three techniques to model and remove these three Galactic foregrounds. 

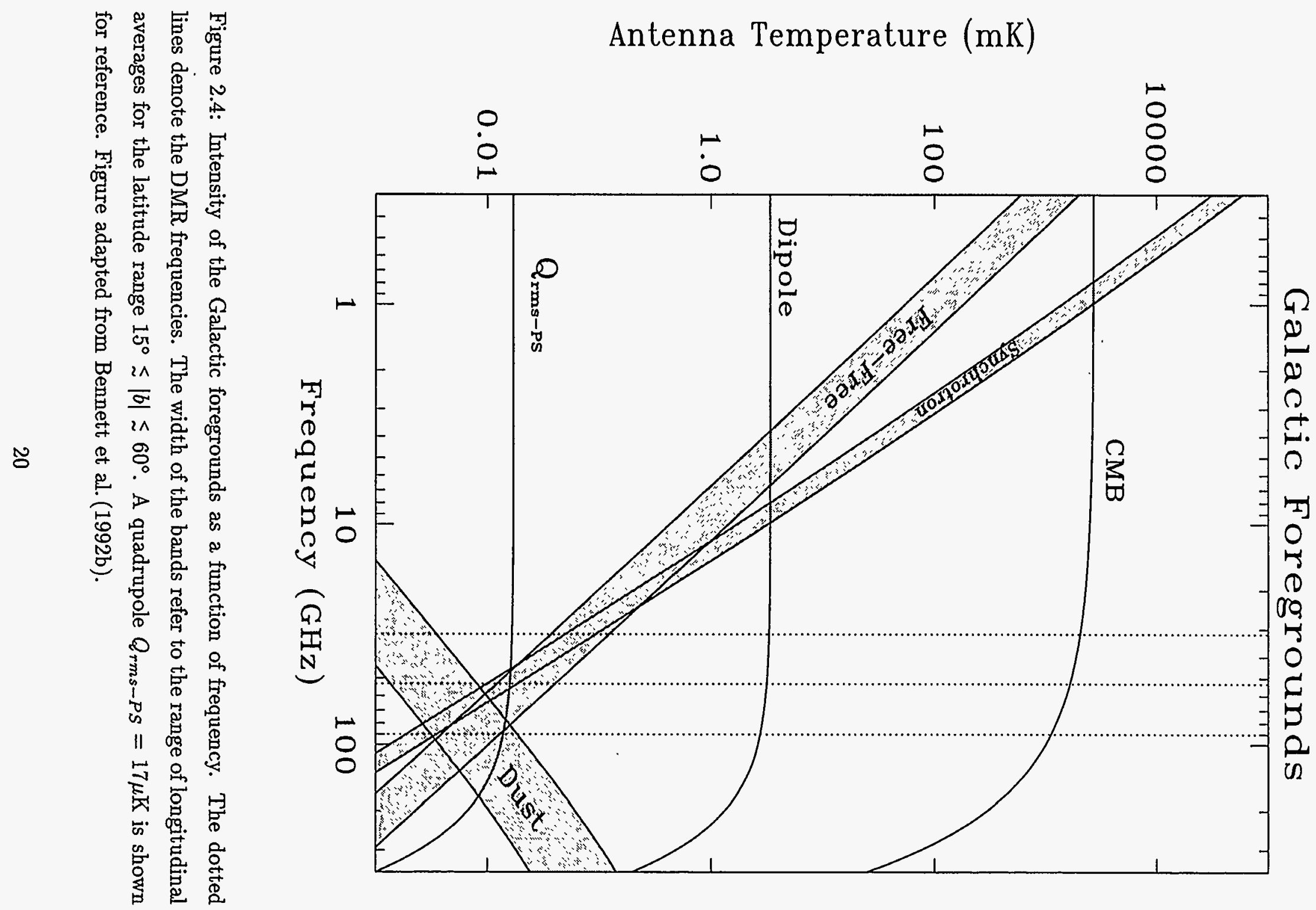


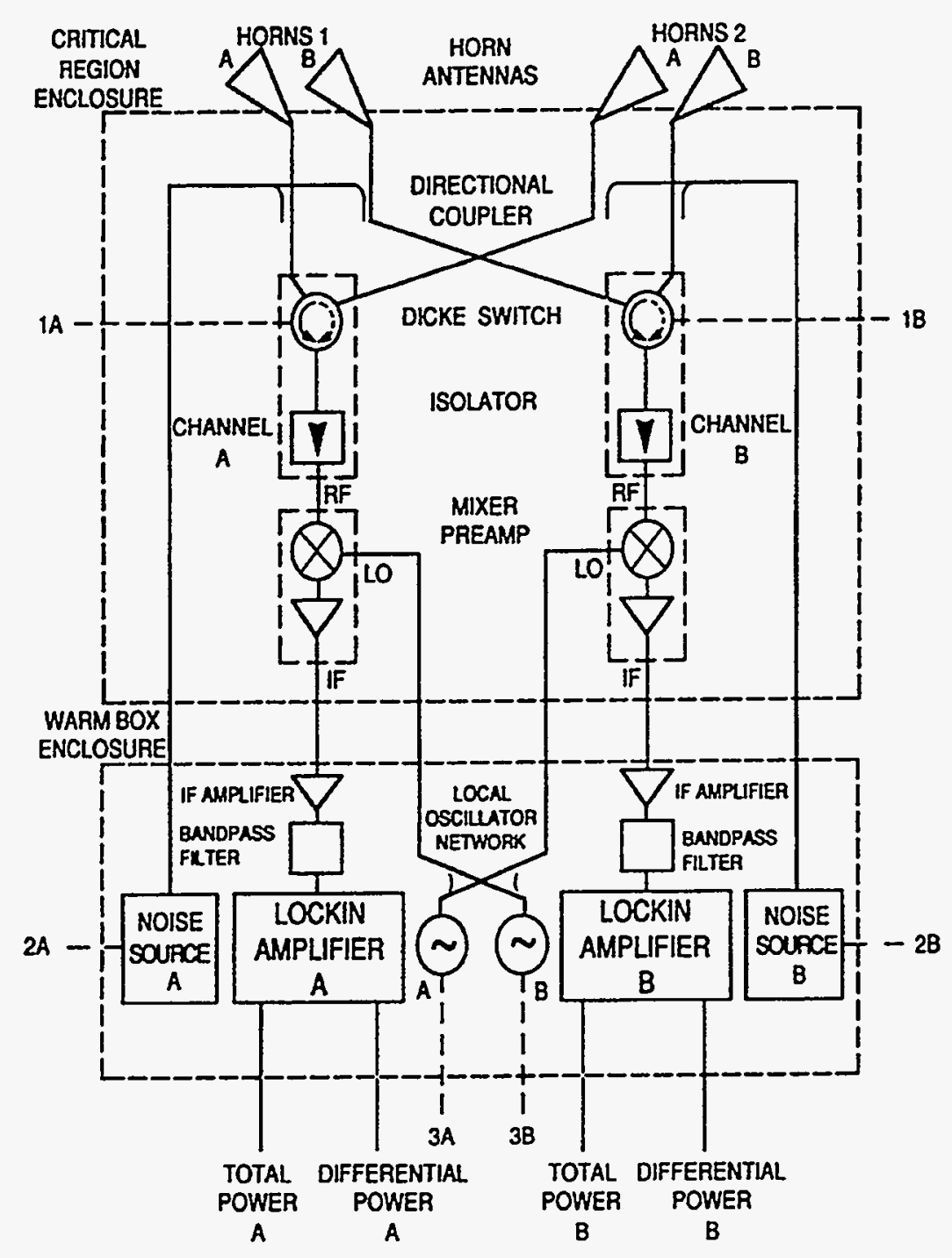

CONTROL FUNCTIONS (AVB AS INDICATED)

1. DICKE SWITCH MODE SELECTION: NORMAL PHASE, INVERSE PHASE,

2. NOISE SOURCE ONOFF LOCK HORN 1, OR LOCK HORN 2

3. LOCAL OSCILLATOR ON/OFF

Figure 2.5: DMR heterodyne receiver. The mixer combines the local oscillator frequency with the Dicke switched signal to create intermediate frequency (IF) beats. These are then amplified, filtered and finally detected by the lock-in amplifier. This schematic of the 53 and $90 \mathrm{GHz}$ radiometers from Smoot et al. (1990). 


\subsection{Instrumentation}

The three frequencies of the DMR, 31.5, 53 and $90 \mathrm{GHz}$, were chosen to span the minimum in the foreground seen in Figure 2.4 and to obtain the largest ratio of CMB signal to Galactic foreground. The bandwidths are 550,850 and $850 \mathrm{MHz}$ respectively. The DMR instruments are heterodyne receivers with diode detectors. Heterodyne refers to the fact that the signal is converted to an intermediate frequency before it is amplified. Figure 2.5 is a schematic of the 53 or $90 \mathrm{GHz}$ radiometers.

Consider a single measurement. Start at time $t=0$ with one horn observing a hot spot of temperature $T_{1}$ while the other horn is pointed at a cooler spot of $T_{2}$. One twohundredth of a second later the Dicke switch changes the input to let the signal from the lower temperature $T_{2}$ through. The Dicke switch alternates between the two horns at $100 \mathrm{~Hz}$. The mixer combines the stable local oscillator frequency with the alternating horn signals creating lower beat frequencies. These beats are then rectified by a diode and processed through a low-pass filter with a DC block isolating beat frequencies in the range 1 to 1.8 $\mathrm{GHz}$ (40-560 MHz for the $31 \mathrm{GHz}$ channels). They are then amplified, rectified and passed through a band pass filter. A lock-in amplifier (square wave detector) with a synchronous filter measures the series of voltage differences, $d V_{i}$, integrates for half a second and then outputs the mean voltage difference which is proportional to the antenna temperature difference seen by the two horns. A half second integration time is a compromise between good pointing (smaller integration the better) and more accurate output. The output is

$$
\Delta V=\sum_{i=1,50} d V_{i} \propto \Delta T_{a n t}=T_{1}-T_{2}
$$

The lock-in amplifier is found to leave a $3.2 \%$ correlation from one half second integration to the next and is corrected for (Kogut et al. 1992, Bennett et al. 1994). See Smoot et al. (1990), Bennett et al. (1992a) and Janssen et al. (1994) for more on the instrumentation.

\subsection{Noise}

To a very good approximation, the noise variance in a single pixel of a map from a give channel can be represented by

$$
\sigma_{i}^{2}=\sigma_{c h}^{2} / N_{i}
$$

where $\sigma_{c h}^{2}$ is the channel dependent instrument noise variance per observation and $N_{i}$ is the number of observations of that pixel (see Chapter 9). The accuracy of equation (2.5) 
and correlated noise is treated in depth in Chapter 9. Noise simulations require $\sigma_{c h}$ as an input. Table 2.1 summarizes various estimates of $\sigma_{c h}$. The "nominal" rms values in Table 2.1 are based on ground calibrations, while the "flight" rms values are based on the value of $\sigma_{c h}$ which make the $\chi^{2} /$ dof of the differential data equal to 1 (see equation (3.6)). The factors used to convert antenna temperature differences to thermodynamic temperature differences for $\nu=31.5,53,90 \mathrm{GHz}$ are respectively 1.026, 1.074, 1.226 (see Appendix G for details). If the variances of two maps are $\sigma_{1}^{2}$ and $\sigma_{2}^{2}$ respectively, then the rms of the unweighted sum maps is computed from $\sigma^{2}=\frac{\sigma_{1}^{2}+\sigma_{2}^{2}}{4}$. For weighted sums, $\sigma^{2}=\frac{1}{1 / \sigma_{1}^{2}+1 / \sigma_{2}^{2}}$ is used. This assumes that the number of observations for the resultant map is defined as $N_{i}=\left(N_{1, i}+N_{2, i}\right) / 2$ and the resulting pixel variance is then $\sigma_{i}^{2}=\sigma^{2} / N_{i}$.

The DMR calibration is described in Bennett et al.(1992a \& 1994). There are three possibilities for calibration:

1) the noise source firings every 2 minutes

2) the dipole seen in the maps based on the precisely known velocity of the Earth

3) the Moon.

The noise source firings are controlable and are used to find the calibration constant to convert voltage output to antenna temperature.

Although the Dicke switches were carefully covered in $\mu$-metal to shield out magnetic fields, the largest systematic error in the DMR maps is the magnetic susceptibility of the Dicke switches as the satellite moves around in the magnetic field of the Earth (Kogut et al. 1992, Bennett et al.1994). The effect of magnetic susceptibility is fit for and partially corrected (equation (3.1)). 
Table 2.1: DMR RMS Noise Values ${ }^{a}$

\begin{tabular}{|r|cc|cc|cc|cc|}
\hline & \multicolumn{2}{|c|}{ Nominal RMS } & \multicolumn{2}{|c|}{ Flight RMS (YR1) } & \multicolumn{2}{|c|}{ Flight RMS (YR2) } & \multicolumn{2}{|c|}{ Flight RMS (2YR) } \\
\hline Map & $\begin{array}{c}T_{\text {ant }}^{d} \\
(\mathrm{mK})\end{array}$ & $\begin{array}{c}T \\
(\mathrm{mK})\end{array}$ & $\begin{array}{c}T_{\text {ant }} \\
(\mathrm{mK})\end{array}$ & $\begin{array}{c}\text { T } \\
(\mathrm{mK})\end{array}$ & $\begin{array}{c}T_{\text {ant }} \\
(\mathrm{mK})\end{array}$ & $\begin{array}{c}T \\
(\mathrm{mK})\end{array}$ & $\begin{array}{c}T_{\text {ant }} \\
(\mathrm{mK})\end{array}$ & $\begin{array}{c}T \\
(\mathrm{mK})\end{array}$ \\
\hline 31A & 55.0 & 56.4 & 59.2 & 60.7 & 58.1 & 59.6 & 58.6 & 60.1 \\
31B & 58.4 & 60.0 & 60.8 & 62.4 & 60.0 & 61.6 & 60.4 & 62.0 \\
53A & 22.5 & 24.2 & 23.2 & 24.9 & 23.2 & 24.9 & 23.2 & 24.9 \\
53B & 24.9 & 26.7 & 27.1 & 29.1 & 27.1 & 29.1 & 27.1 & 29.1 \\
90A & 40.3 & 49.4 & 40.0 & 49.0 & 39.4 & 48.3 & 39.7 & 48.7 \\
90B & 28.3 & 34.7 & 29.9 & 36.7 & 30.5 & 37.4 & 30.2 & 37.0 \\
\hline 31A+B & 40.1 & 41.1 & 42.4 & 43.5 & 41.7 & 42.8 & 42.1 & 43.2 \\
31A+Bw & 40.0 & 41.0 & 42.4 & 43.5 & 41.7 & 42.8 & 42.1 & 43.2 \\
$53 \mathrm{~A}+\mathrm{B}$ & 16.8 & 18.0 & 17.8 & 19.1 & 17.8 & 19.1 & 17.8 & 19.1 \\
$53 \mathrm{~A}+\mathrm{Bw}$ & 16.7 & 17.9 & 17.6 & 18.9 & 17.6 & 18.9 & 17.6 & 18.9 \\
90A+B & 24.6 & 30.2 & 25.0 & 30.7 & 24.9 & 30.6 & 25.0 & 30.6 \\
90A+Bw & 23.2 & 28.4 & 24.0 & 29.5 & 24.1 & 29.6 & 24.0 & 29.4 \\
\hline
\end{tabular}

a The variance per observation is the square of these rms values.

$b$ "Nominal" rms values are based on ground calibrations.

c "Flight" rms values are based on the value of $\sigma_{c h}$ which will make the $\chi^{2} /$ dof of the differential data equal to 1 (see equation (3.6)).

$d$ The factors used to convert antenna temperature differences to thermodynamic temperature differences for $\nu=31.5,53,90 \mathrm{GHz}$ are respectively $1.026,1.074,1.226$ (see Appendix $\mathrm{G}$ for details). The single channel rms values have been provided by $\mathrm{Phil}$ Keegstra. $e$ "w" indicates a weighted sum. 


\title{
Chapter 3
}

\section{Data Processing}

\begin{abstract}
The main goal of the data processing is to turn the differential measurements into a sky map. In this chapter we provide an overview of the software pipeline in which the data flow from the satellite to the maps. We present a description of the calibration, baseline removal and correction of the data which are then used to make the maps. We concentrate on describing how the map-making code operates.
\end{abstract}

\subsection{Differential Data}

The measurements are stored on the satellite on magnetic tapes. Once a day for ten minutes as the satellite passes over the east coast of the United States, the stored data is relayed to a ground station on Wallops Island and then to Goddard Space Flight Center. The DMR data is stripped from the COBE telemetry stream and merged with attitude and orbit information. Data known or suspected to be erroneous are flagged.

During the $m$ th half second observation ( $1 \leqq m \leqq m_{\text {tot }}$, where $m_{\text {tot }}$ is the the total number of observations for the channel under consideration), the positive horn is pointing in a direction indexed by $m+$, while the negative horn is pointing $60^{\circ}$ away in a direction indexed by $m-$. The $m$ th uncalibrated and uncorrected raw measurement can be modeled (Kogut et al. 1992) as

$$
S(m)=\frac{1}{\mathcal{G}(m)}\left[\Delta T(m)+\Delta(m)+O(m)+\sum_{k} W_{k}(m)+\sum_{q} E_{q} f_{m q}\right]
$$


where $\mathcal{G}(m)$ is the true instrumental gain factor in digital units per antenna temperature, $\Delta T(m)$ is the true temperature difference $T(m+)-T(m-), \Delta(m)$ is the instrument noise with a variance of $\sigma_{m}^{2}, O(m)$ is the offset produced by small imbalances in the differential radiometer and the $W_{k}(m)$ are a series of "well-known" time-dependent non-cosmological signals including the Doppler effect of the satellite's velocity as well as the Earth's velocity. Less well known instrumental effects such as the effect of the Earth's magnetic field on the DMR are represented by the $E_{q} f_{m q}$ 's. The amplitudes $E_{q}$ are poorly known but the time-dependences, $f_{m q}$, are fairly well known ( $m$ is a function of time).

We are only interested in $\Delta T(m)$ so the other factors need to be estimated and removed as accurately as possible. We remove the offset $O(m)$ by subtracting the derived baseline $B(m)$. We calibrate the measurement by multiplying by a gain $\mathcal{G}^{\prime}(m)$ derived from the data (Bennett et al.1992a, 1994). To remove the effect of the well known $W_{k}$, we compute $W_{k^{\prime}}^{\prime}(m)$ from pointing information and a knowledge (satellite velocity, Earth velocity) and subtract it. To summarize, we calibrate and correct for the true unknown $\mathcal{G}(m), O(m)$, and $W_{k}(m)$ 's with values $\mathcal{G}^{\prime}(m), B(m)$ and $W_{k^{\prime}}^{\prime}(m)^{\prime}$ 's derived from the data and a priori knowledge. We can therefore represent the calibrated, offset removed, corrected data as

$$
D(m)=\mathcal{G}^{\prime}(m)[S(m)-B(m)]-\sum_{k^{\prime}} W_{k^{\prime}}^{\prime}(m)
$$

combining equation (3.1) and (3.2) and regrouping yields

$D(m)=\frac{\mathcal{G}^{\prime}(m)}{\mathcal{G}(m)}\left[\Delta T(m)+\sum_{q} E_{q} f_{m q}+\Delta(m)\right]+\left[\frac{\mathcal{G}^{\prime}(m)}{\mathcal{G}(m)} \sum_{k} W_{k}-\sum_{k^{\prime}} W_{k^{\prime}}^{\prime}\right]+\mathcal{G}^{\prime}(m)\left[\frac{O(m)}{\mathcal{G}(m)}-B(m)\right]$.

We treat the last two terms as residual noise. The gain estimate is good to a few percent (Bennett et al. 1994) i.e., $0.97 \lesssim \frac{\mathcal{G}^{\prime}(m)}{\mathcal{G}(m)} \lesssim 1.03$. We are left with

$$
D(m)=\Delta T(m)+\sum_{q} E_{q} f_{m q}+\Delta(m)
$$

This procedure has turned the raw measurements $S(m)$ into the calibrated, baseline removed and well-known systematic corrected differential measurements $D(m)$ ready for input into the map-making algorithm. If there are any errors in those procedures that correlate with position on the sky, they will create spurious signals in the maps. The list of $W_{k^{\prime}}^{\prime}(m)$ and $E_{q}$ are given in Appendix E.

One can conveniently represent the $m_{\text {tot }}$ dimensional vector $\vec{D}$ from equation (3.4) in 
matrix form as

$$
\left(\begin{array}{c}
D_{1} \\
D_{2} \\
D_{3} \\
D_{4} \\
D_{5} \\
D_{5} \\
\vdots
\end{array}\right)=\left(\begin{array}{cccccccccccccc}
0 & 1 & 0 & 0 & \ldots & 0 & -1 & 0 & 0 & \ldots & 0 & f_{11} & \ldots & f_{1 Q} \\
1 & 0 & 0 & 0 & \ldots & 0 & 0 & -1 & 0 & \ldots & 0 & f_{21} & \ldots & f_{2 Q} \\
0 & 0 & -1 & 0 & \ldots & 0 & 1 & 0 & 0 & \ldots & 0 & f_{31} & \ldots & f_{3 Q} \\
& & & & & & & \bullet & & & & & & \\
& & & & & & & & \bullet & & & & & \\
& & & & & & & \bullet & & & & & & \\
\vdots \\
T_{6144} \\
E_{1} \\
\vdots \\
E_{Q}
\end{array}\right)+\left(\begin{array}{c}
T_{1} \\
T_{2} \\
\Delta_{4} \\
\Delta_{5} \\
\Delta_{6} \\
\vdots
\end{array}\right)
$$

or

$$
\vec{D}=\mathrm{V} \vec{t}+\vec{\Delta}
$$

where $V$ is an $m_{\text {tot }} \times(6144+Q)$ design matrix containing the pointing information and $\vec{t}$ contains the sky map and the $Q$ coefficients we want to solve for. Notice that the $1,-1$ pairs in each row of $\mathrm{V}$ create temperature differences out of the temperatures.

We solve for the coefficients $E_{q}$ at the same time we solve for the temperatures by minimizing the $\chi^{2}$. Thus fitting for the $E_{q}$ 's removes the best-fit signal from the maps which is consistent with the assumed time dependence $f_{m q}$ (see Janssen \& Gulkis 1992).

We refer to the $W_{k}$ 's as corrections and the $E_{q}$ 's as fits. So typically we "correct" for the satellite's velocity and "fit" for magnetic susceptibility coefficients. The dichotomy between corrections and fits is not fundamental. Once we have $E_{q}$ 's we trust, we can use them as corrections the next time we run the program. Additionally we can choose not to correct for a known effect or we can fit for it. The code is set up to handle multiple output maps also known as multiple right hand sides, i.e., the sums over $q$ and $k^{\prime}$ in equation (3.3) and (3.4) can be varied to yield maps with or without certain corrections and fits. For diagnostic purposes we have run 40 right hand sides for pass 1 and 68 right hand sides for pass 2 (see Appendix $\mathrm{E}$ for a description of pass 1 and pass 2). The advantage of this is that we can compare two maps, for example one corrected for the magnetic effect and one not corrected. The difference is an estimate of the size of the effect. Figure 2 of Kogut et al. (1992) is an example of a magnetic systematic error correction found in this way. See Appendix E and Bennett et al.(1994) for the corrections and fits which were performed on the pass 1 and pass 2 maps. For convenience in the following section we specialize to the case in which no fits for systematic errors are made. There is no loss of generality in this since good fits can be treated as corrections in the subsequent processing of the data. 


\subsection{Map-Making Algorithm}

We form a map by minimizing $\chi^{2}$ defined as

$$
\chi^{2} \equiv \sum_{m=1}^{m_{\text {tot }}} \frac{(D(m)-\Delta T(m))^{2}}{\sigma_{m}^{2}}
$$

Setting $\frac{\partial \chi^{2}}{\partial T_{k}}=0$ yields the normal equations

$$
\mathrm{A} \vec{T}_{o b s}=\vec{M}
$$

where $\vec{T}_{\text {obs }}$ is the least-squares estimate of the true temperatures $\vec{T}, \mathbf{A}=\mathbf{V}^{T} \mathbf{\Sigma V}$ where $\boldsymbol{\Sigma}$ is an $m_{\text {tot }} \times m_{\text {tot }}$ diagonal matrix with $\Sigma_{m m}=1 / \sigma_{m}^{2}$, and $\vec{M}=\mathrm{V}^{T} \Sigma \vec{D}$ is the measurement vector. Since $\sigma_{m}$ is stable and varies only slightly with time we use $\sigma_{m}^{2}=\sigma_{c h}^{2}$ : the measurement variances are equal to the channel dependent variance of the instrument noise. The effect of using a time independent $\sigma_{c h}$ instead of $\sigma_{m}$ is that each measurement for that channel has the same weight. A measure of the year to year variation in $\sigma_{m}$ can be obtained by comparing column 4 with column 6 in Table 2.1. The percentage changes of the flight rms from year 1 to year 2 are $-1.8,-1.3,0.0,0.0,-1.5$, and +2.0 for channels $31 \mathrm{~A}$, $31 \mathrm{~B}, 53 \mathrm{~A}, 53 \mathrm{~B}, 90 \mathrm{~A}$ and $90 \mathrm{~B}$ respectively. Thus the variation in $\sigma_{m}$ is less than $2 \%$ for all channels and negligible for the $53 \mathrm{~A}$ and $\mathrm{B}$ maps. Thus $\sigma_{m} \approx \sigma_{c h}$ and the multiplicative factor $1 / \sigma_{c h}^{2}$ cancels in equation (3.7). This permits the convenient redefinition of $A$ to the dimensionless $\mathbf{A}=\mathbf{V}^{T} \mathbf{V}$.

The matrix $\mathrm{A}$ is $6144 \times 6144$, symmetric, sparse, positive semi-definite and formally singular due to the differential nature of the observations. We solve the normal equations by augmenting the diagonal terms of $\mathbf{A}$ by a small positive number $\epsilon$. This does nothing more than impose an arbitrary mean level on an otherwise unique solution. This point is discussed in detail in Appendix J. A Gauss-Seidel procedure finds an iterative solution to the normal equations and gives the desired best-fit sky map $\vec{T}_{\text {obs }}$. Gauss-Seidel iterative procedures are particularly well suited to invert large sparse matrices since the number of stored parameters and computations required depends only on the number of non-zero matrix elements. The Gauss-Seidel procedure used is a modified Jacobi iterative technique in which the new solutions computed during an iteration are used in later computations within the same iteration. Each iteration provides a solution vector which converges toward the best fit solution to the data (see Figures 3.1-3.4). For further details on how the maps are made see Torres et al. (1988), Smoot et al. (1990), Keegstra et al. (1991), Jackson et al. (1991) Janssen \& Gulkis (1992) and Wright (1994b). 
We combine equations (3.5) and (3.7), with the definition $\vec{\delta} \equiv \mathrm{V}^{T} \vec{\Delta}$ to write the best-fit temperatures as a function of the true temperatures plus a noise term $\vec{n}$,

$$
\vec{T}_{\text {obs }}=\vec{T}+\vec{n}=\vec{T}+\mathbf{A}^{-1} \vec{\delta}
$$

Although the components of $\vec{\delta}$ individually come from Gaussian distributions of variance $N_{i} \sigma_{c h}^{2}$, those separated by $60^{\circ}$ from each other are anticorrelated because the same $\Delta(m)$ contributes positively to one pixel and negatively to the other. The analysis of the correlations in the DMR maps due to the noise term $\vec{n}=\mathrm{A}^{-1} \vec{\delta}$ is the subject of Lineweaver et al. (1994). Chapter 9 is an extended and more detailed version of this paper.

\subsubsection{Pointing and Pixelization}

There are two sets of attitude which determine the accuracy of the pointing (Kumar et al. 1991, Wright et al.1991b). Coarse attitude is derived from Sun and Earth sensors on the spacecraft and has a nominal accuracy of better than $0.5^{\circ}$, however a typical accuracy of $0.1^{\circ}$ is achieved for the two year data. DIRBE fine aspect attitude is the result of matching DIRBE stars to a star catalogue and has a nominal accuracy of $0.1^{\circ}$.

Pixelization of the COBE data sets are standardized using the quadrilateralized spherical cube discussed in Chan \& O'Neil (1975), Hon (1991) and White \& Stemwedel (1992). The paradoxical neologism "spherical cube" can be simply understood. Place a $32 \times 32(=1024)$ pixel grid on each of the six faces of a cube $(6$ faces $\times 1024$ pixels $=6144$ pixels). Place the cube inside a sphere. A point source of light at the center of the cube projects each of the cube pixels onto the sphere. An appropriate deformation of the pixel grid on the cube faces produces pixels of approximately equal area on the sphere.

There are 41,253 square degrees in the $4 \pi$ steradians of the sky. With 6144 pixels, the average pixel size is $\sqrt{41253 / 6144}=2.59^{\circ}$. The areas of the largest and smallest pixels are about $3 \%$ larger and smaller than average with a rms area fluctuation of about $1 \%$. The FIRAS and DMR instruments both have approximately $7^{\circ} \mathrm{FWHM}$ beams, while the DIRBE instrument has a square $0.7^{\circ} \times 0.7^{\circ}$ field of view. The FIRAS and DMR beams are oversampled by the chosen standard $2.59^{\circ} \times 2.59^{\circ}$ pixels. Thus the DMR maps oversample the DMR beam by factor of about $3\left(\approx 7^{\circ} / 2.6^{\circ}\right)$. The number of pixels in a map, $N_{\text {pix }}$, is easily increased or decreased by changing the "resolution" of the map defined by

$$
N_{\text {pix }} \equiv 6 \times\left[2^{(\text {resolution-1) }}\right]^{2} \text {. }
$$


Setting resolution $=6$ produces 6144 pixels and is the most common DMR format. Conversion to different resolutions is often done for analysis purposes. In the pass 2 version of the map-making algorithm a split resolution was chosen. In the Galactic plane where the signal to noise is high, the resolution of the map is increased by one and thus the number of pixels in the $|b|<20^{\circ}$ region is four times larger. The region $|b|<20^{\circ}$ is $\left(100 \times \sin 20^{\circ}\right) \%$ of the sky. Therefore DMR split resolution maps have $4032\left(\approx\left(1-\sin 20^{\circ}\right) \times 6144\right)$ pixels outside the cut and $8448\left(\approx 4 \times \sin 20^{\circ} \times 6144\right)$ pixels in the Galactic plane for a total of 12480 pixels.

No pixelization is assumed in the data processing until the pointing matrix $\mathrm{V}$ in equation (3.5) is constructed by the map-making algorithm.

\subsubsection{Validation}

There may be spikes in the data which could come from noise or from an instrument glitch. As an example of one of many tests performed to validate the map-making procedure Figures 3.1-3.4 show how a large spike in the data is handled by the map-making algorithm at various stages of the iteration procedure. The central pixel was given a value of 10000 $\mathrm{mK}$ during one observation. Other observations of the same pixel were left unchanged. Figures 3.1-3.4 are snap shots after the 1st, 2nd, 5th and 30th iterations of the map making algorithm. The data processed here contain full sky coverage from only 16 days of observations chosen from moonless periods at different times of year.

The six cube faces are apparent in Figures 3.3 and 3.4. The cube faces are indexed 0 through 5 . Face 0 and 5 cover the north and south poles respectively. Face 1 is in the center, face 2 is centered at $\ell=+90$, face 3 is centered at $\ell=180$ and face 4 is centered at $\ell=-90$. The contrast between faces 0 and 4 and faces 1 and 5 is due to the Gauss-Seidel procedure which continually updates the solutions during an iteration; whenever the solutions for the temperatures are changing by a noticeable amount during an iteration, the first faces can have mean levels different from the later faces. This can be seen in the maps when the greyscale interval is appropriately small. 


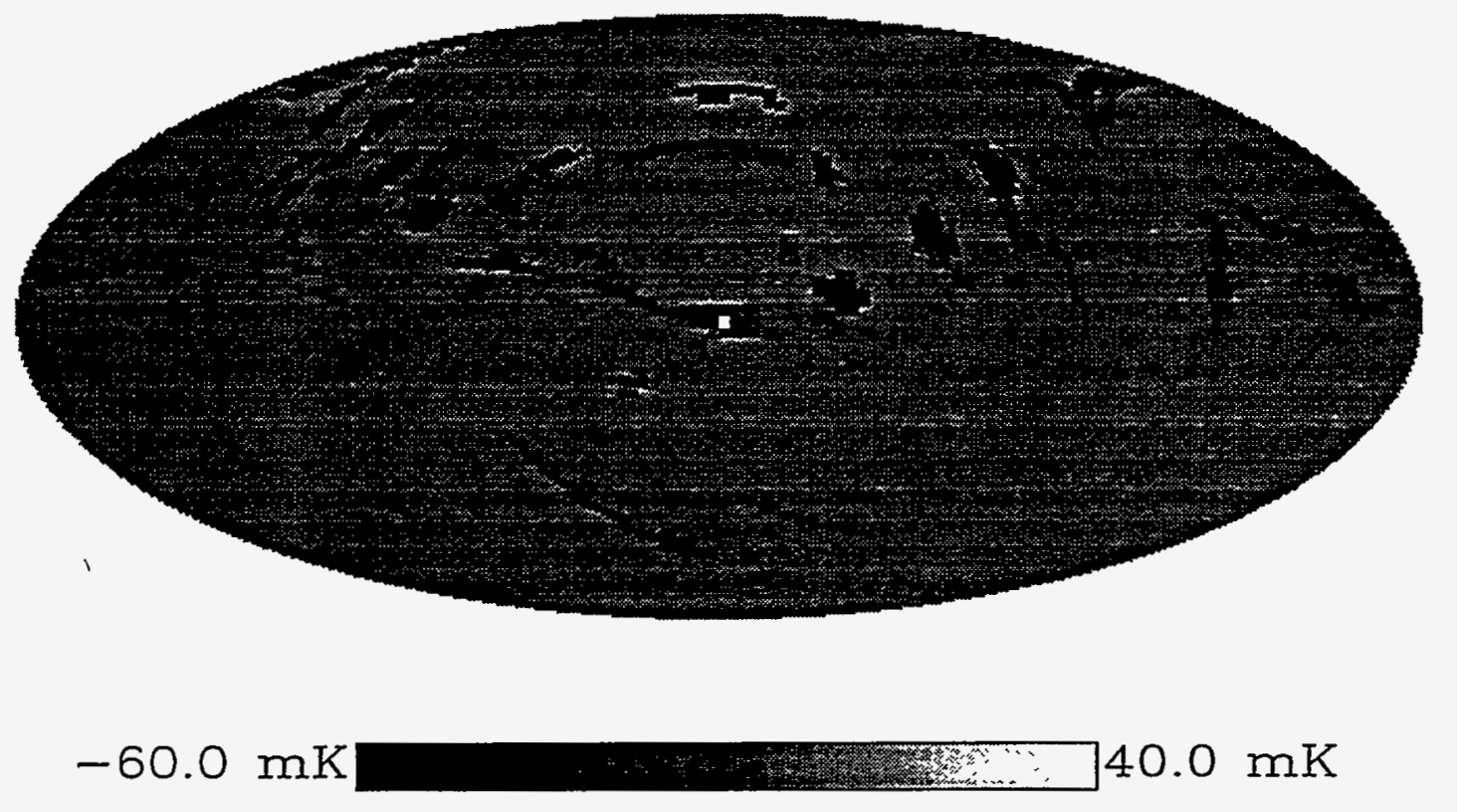

Figure 3.1: Effect of Noise Spike on the Map Solution: 1st Iteration.

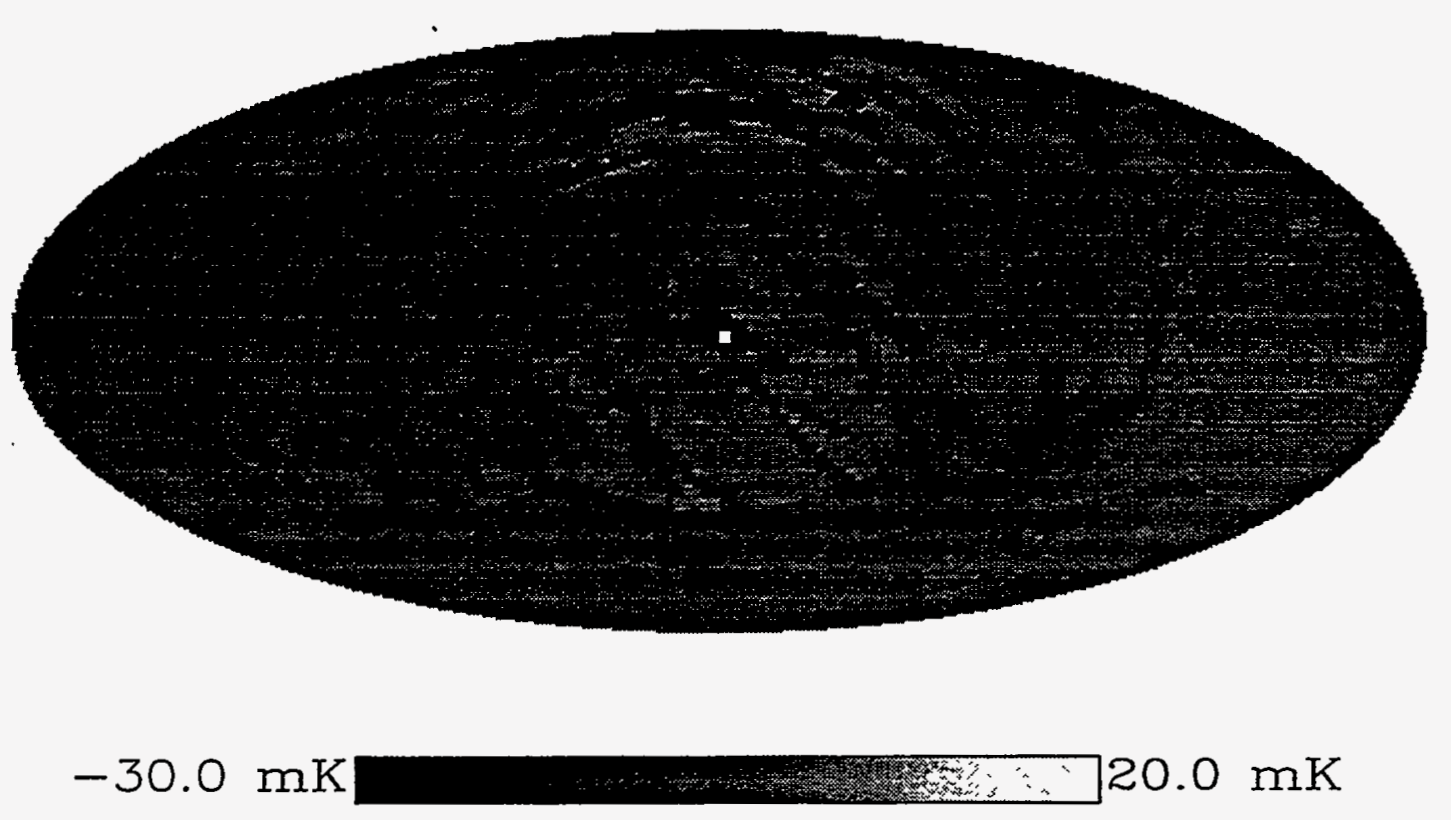

Figure 3.2: Effect of Noise Spike on the Map Solution: 2nd Iteration. 

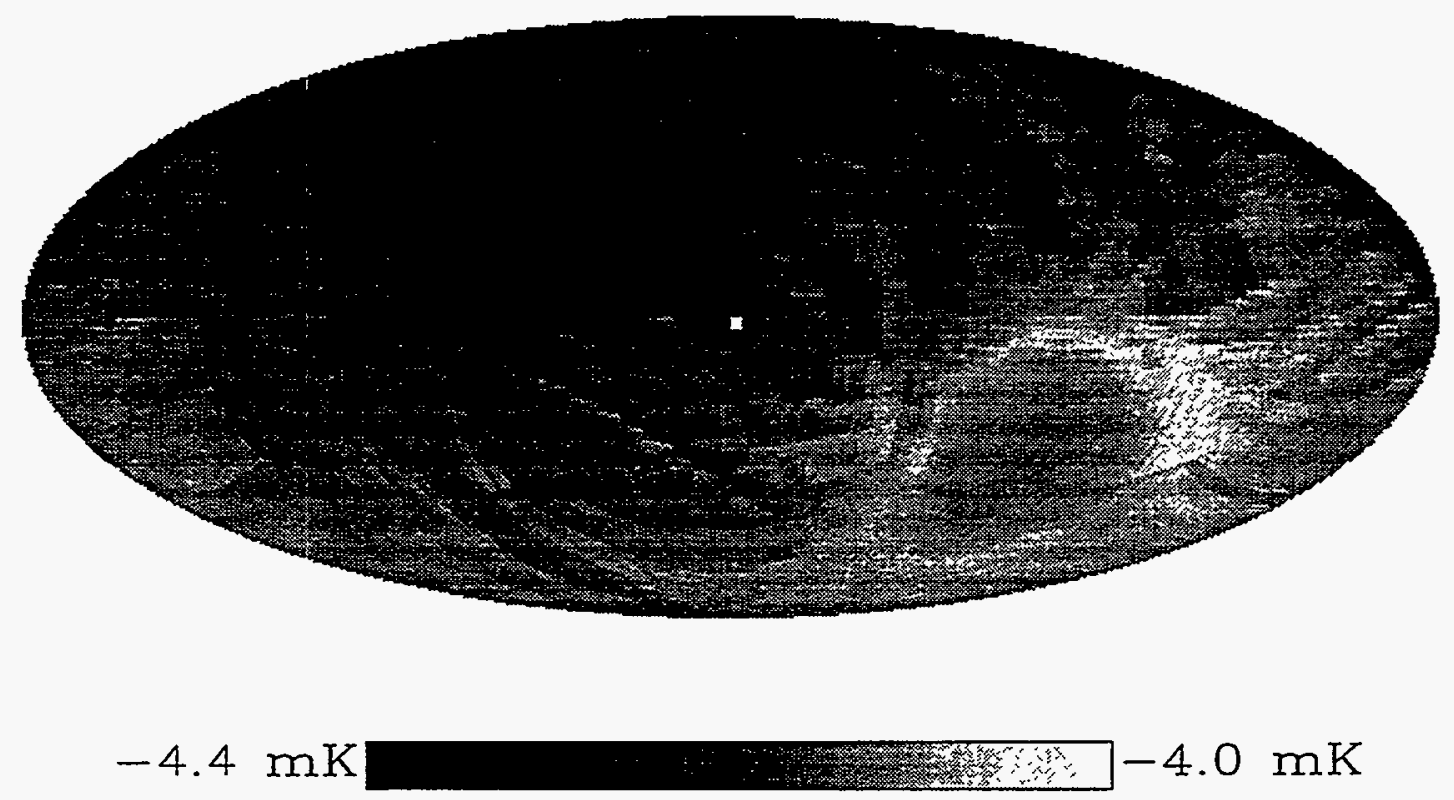

Figure 3.3: Effect of Noise Spike on the Map Solution: 5th Iteration.

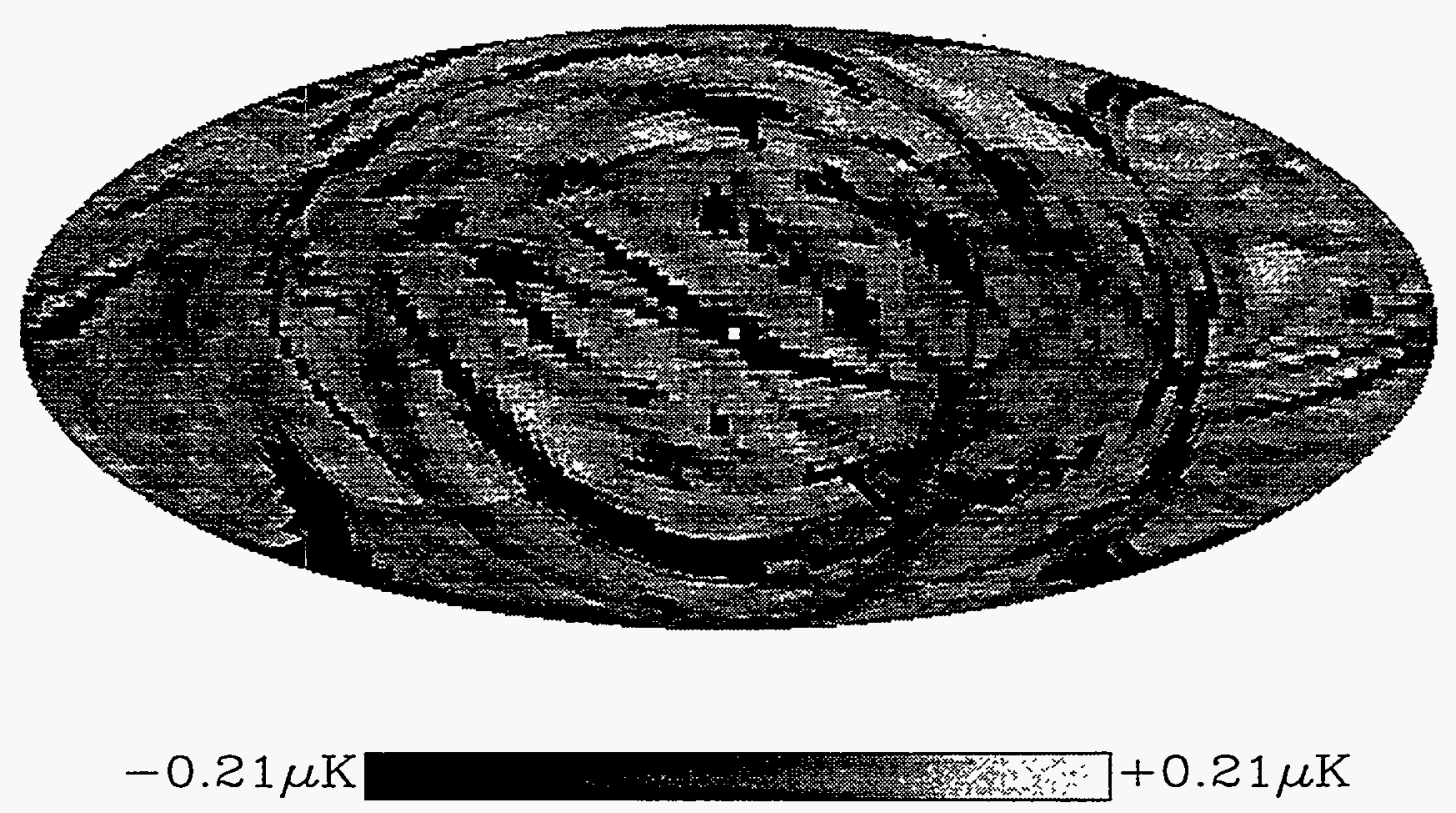

Figure 3.4: Effect of Noise Spike on the Map Solution: 30th Iteration. Note the presence of the broken cool ring at $60^{\circ}$ and $120^{\circ}$ angular separation from the central pixel (compare this to Figure 9.2). The breaks in the rings are due to the unevenness of the sky coverage in the 16 days of data used. In contrast to the previous three figures, the mean level of the map $(-4.19254 \mathrm{mK})$ has been subtracted to show the variation around the mean. 


\title{
Chapter 4
}

\section{Correlation Function: Theory versus Practice}

\begin{abstract}
The two-point temperature correlation function provides an important characterization of CMB anisotropies. We present several simple examples to illustrate the use and power of a correlation function analysis. We describe its relation to the power spectrum and the role played by cosmic variance. We compare the theory to the nitty-gritty of the data analysis including incomplete sky coverage and the real DMR beam pattern. Substantial underestimates for the true rms fluctuations and for $C\left(\alpha<10^{\circ}\right)$ result if a Gaussian approximation to the DMR beam is used. The error in $C(0)$ is $\approx 150 \mu \mathrm{K}^{2}$. Biases in the auto-correlation due to multipole subtractions are in the range 20 to $70 \mu \mathrm{K}^{2}$. Additionally we describe a novel way to obtain the quadrupole amplitude of our observable Universe from the correlation function.
\end{abstract}

\subsection{Introduction}

The two-point angular correlation function $C(\alpha)$ is a powerful way to analyze the statistical properties of a two-dimensional sky map. If the temperatures of the cosmic microwave background sky $\mathrm{T}(\theta, \phi)$ are from a random phase Gaussian distribution then the quadratic statistic $C(\alpha)$ completely describes the statistical properties of $\mathrm{T}(\theta, \phi)$. This follows from the fact that all odd moments of a Gaussian distribution disappear, while all 
even moments are functions of the second moment. The results of Smoot et al. (1994) are consistent with the temperatures being Gaussian. If $\mathrm{T}(\theta, \phi)$ is non-Gaussian, $C(\alpha)$ tells us the second moment while the third moment is provided by the three-point correlation function (Hinshaw et al. 1994).

The correlation function is a multipurpose statistic. Correlation functions of the DMR data can be easily compared with cosmological models or with the correlation functions of other data sets (e.g. Ganga et al. 1993). The power spectrum parameters $n$ and $Q_{r m s-P S}$ can be determined from the cross-correlation functions. Various Galactic latitude cuts can be used to estimate the contribution of the Galaxy to the observed signal (Section 5.3). The instrument noise can easily be compared to the signal and can yield the signal to noise detection level. The sky rms fluctuation level, possibly the most important statistic, is a subset of the correlation function: $\sigma_{s k y}^{2}=C(0)$ (Section 4.7). Correlation functions can be used in the systematic error analysis (Kogut et al. 1992). The correlation functions can also be used to obtain the quadrupole of the observable sky $Q_{r m s}$, as described in Section 4.8 .

\subsection{Legendre Polynomial Expansion}

The two-point correlation function of a sky map is the average product of sky temperatures separated by a given angle $\alpha$

$$
C(\alpha) \equiv<\delta T(\hat{i}) \delta T(\hat{j})>
$$

where $\delta T(\hat{i}) \equiv T_{\text {obs }, i}-\bar{T}$ and the brackets indicate average over all pairs of directions $\hat{i} \hat{j}$, separated by $\alpha_{i j}=\alpha$. Any arbitrary sky temperature map can be expanded in spherical harmonics,

$$
\delta T(\theta, \phi)=\sum a_{\ell m} Y_{\ell m}
$$

Using equation (4.2) in equation (4.1) and employing the addition theorem of spherical harmonics (Appendix I) one obtains

$$
\begin{gathered}
C(\alpha)=\frac{1}{4 \pi} \sum_{\ell_{\min }}^{\ell_{\max }}\left|a_{\ell m}\right|^{2} P_{\ell}(\cos \alpha)=\sum_{\ell_{\min }}^{\ell_{\max }} \Delta T_{\ell}^{2} P_{\ell}(\cos \alpha) \\
<C(\alpha)\rangle=\frac{1}{4 \pi} \sum_{\ell_{\min }}^{\ell_{\max }}\left\langle\left|a_{\ell m}\right|^{2}>P_{\ell}(\cos \alpha)=\sum_{\ell_{\min }}^{\ell_{\max }}<\Delta T_{\ell}^{2}>P_{\ell}(\cos \alpha),\right.
\end{gathered}
$$

where the angle brackets is an ensemble average over all possible model skys, while no brackets refers to the observed values. In equations (4.3), (4.4) and in this thesis we use 
the power spectrum notation

$$
\begin{aligned}
\Delta T_{\ell}^{2} & =\frac{1}{4 \pi} \sum_{m=-\ell}^{\ell}\left|a_{\ell m}\right|^{2}=\frac{1}{4 \pi} \sum_{m=-\ell}^{\ell} b_{\ell m}{ }^{2} \\
\left\langle\Delta T_{\ell}^{2}\right\rangle & =\frac{1}{4 \pi} \sum_{m=-\ell}^{\ell}\left\langle\left|a_{\ell m}\right|^{2}\right\rangle=\frac{1}{4 \pi} \sum_{m=-\ell}^{\ell}\left\langle b_{\ell m}{ }^{2}\right\rangle .
\end{aligned}
$$

See Appendix $H$ for the relation between the complex coefficients $a_{\ell m}$ and the real coefficients $b_{\ell m}$. For the particular case of the quadrupole we have

$$
\Delta T_{2}^{2}=\frac{1}{4 \pi} \sum_{m=-2}^{2}\left|a_{2, m}\right|^{2}=\frac{1}{4 \pi} \sum_{m=-2}^{2} b_{2, m}^{2}=Q_{r m s^{\circ}}^{2}
$$

Appendix $\mathrm{D}$ documents the much wider range of notation found in the literature e.g., $C_{\ell}$, $a_{\ell}^{2},\left(\Delta T / T_{o}\right)_{\ell}^{2}$ and $\epsilon_{H}^{2}$. The $P_{\ell}(\cos \alpha)$ in equation (4.3) are the Legendre polynomials. The first six Legendre polynomials are plotted in the top panel of Figure 4.1.

\subsection{Model Power Spectra}

We are interested in modeling anisotropies on the largest scales down to the $\sim 7^{\circ}$ scales probed by the DMR. At large angular scales where the Sachs-Wolfe or isocurvature effects are important, the CMB power spectrum as a function of the mass-density power spectrum $P(k)$ is

$$
\Delta T_{\ell}^{2}=T_{o}^{2} \frac{2 \ell+1}{8 \pi^{2}}\left(\frac{H_{o}}{c}\right)^{4} \int_{0}^{\infty} \frac{d k}{k^{2}} P(k)\left|j_{\ell}(k \tau)\right|^{2}
$$

where $k$ is the co-moving wavenumber, $\tau$ is the conformal time, $\mathrm{V}$ is the volume normalization and $j_{\ell}$ are the spherical Bessel functions. This integral is the result of considering only the dominant term for large wavelengths $\left(\propto \frac{1}{k}\right)$ in the solution to the collisionless Boltzmann equation (Efstathiou 1990). It is therefore an approximation valid at scales in which the Sachs-Wolfe term dominates other contributions to the anisotropy $(\ell \lesssim 40)$. Terms that contribute to the mean and dipole were also ignored and thus we are considering $\ell>1$ solutions.

If we assume a power law for the mass over-density power spectrum

$$
P(k)=A k^{n}
$$

where $n$ is the power spectral index and $\mathrm{A}$ is a normalization constant with dimensions length $h^{n+3}$ to insure that the power spectrum $P(k)$ has the dimensions of volume. We can 

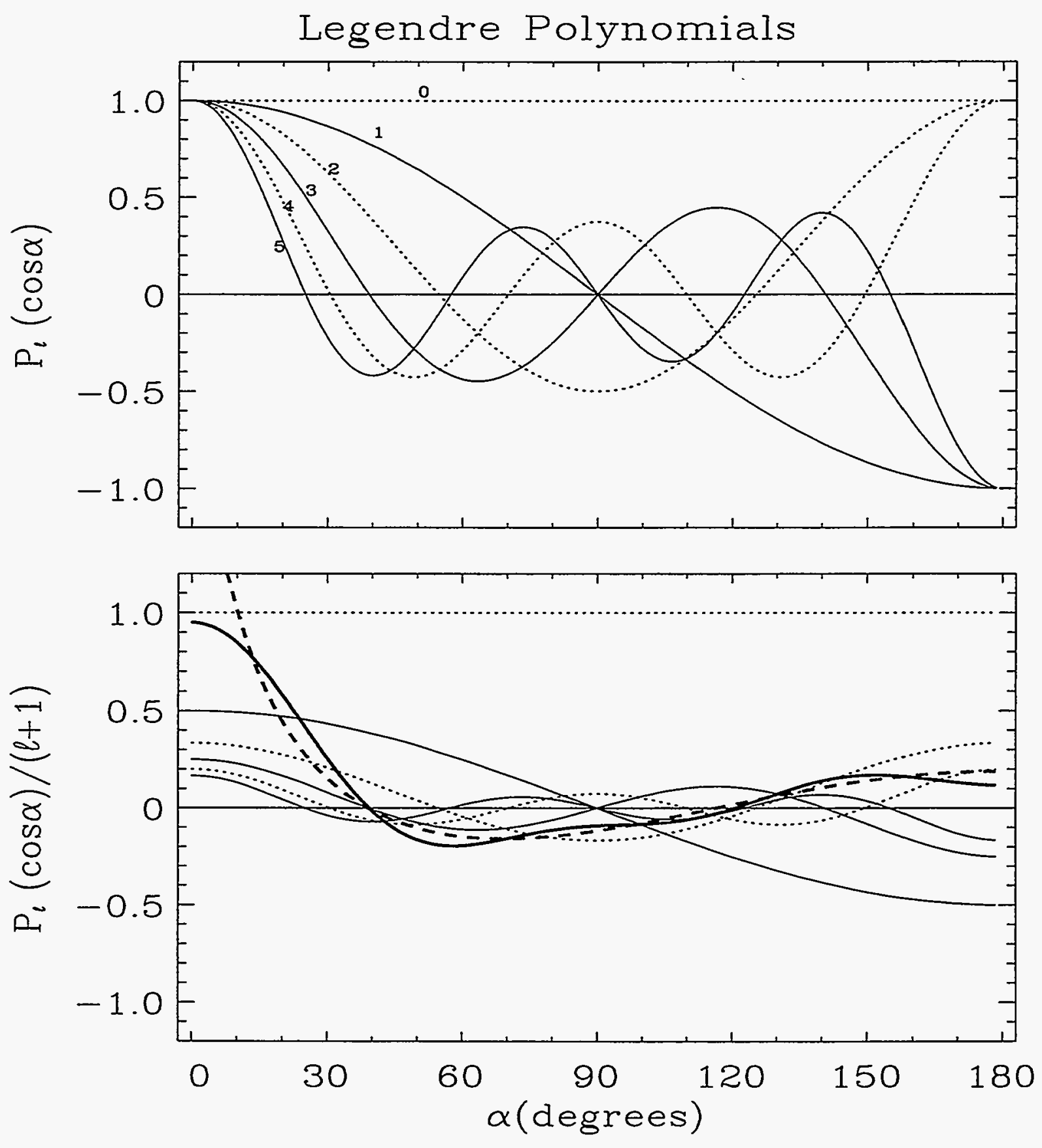

Figure 4.1: Legendre Polynomial Expansion of the Correlation Function. The Legendre polynomials for $\ell=0$ to 5 are labelled in the top panel. Harrison-Zeldovich weightings of the same Legendre polynomials are shown in the bottom panel. The thick solid line is the sum for $2 \leq \ell \leq 5$. The thick dashed line is for $2 \leq \ell \leq 70$. Notice that $C\left(\alpha \gtrsim 30^{\circ}\right)$ is well approximated by only the first four $\ell$ values. 
evaluate the integral of equation (4.8) and obtain

$$
<\Delta T_{\ell}^{2}>=T_{0}^{2}\left(\frac{2 \ell+1}{4 \pi}\right) \frac{A}{16}\left(\frac{H_{0}}{c}\right)^{n+3} \frac{\Gamma(3-n)}{\Gamma^{2}\left(\frac{4-n}{2}\right)} \frac{\Gamma\left(\ell+\frac{n-1}{2}\right)}{\Gamma\left(\ell+\frac{5-n}{2}\right)} .
$$

This is the exact solution of the integral provided $n<3$. For $n \geq 3$ equation (4.8) diverges for high values of $k$ since $\lim _{k \rightarrow \infty}\left|j_{\ell}(k \tau)\right|^{2}=\frac{1}{k}$. We can write equation (4.10) in terms of the quadrupole moment $\Delta T_{2}^{2} \equiv Q_{\tau m s-P S}^{2}$

$$
<\Delta T_{\ell}^{2}\left(n, Q_{r m s-P S}\right)>=Q_{r m s-P S}^{2} \frac{(2 \ell+1)}{5} \frac{\Gamma\left(\ell+\frac{n-1}{2}\right) \Gamma\left(\frac{9-n}{2}\right)}{\Gamma\left(\ell+\frac{5-n}{2}\right) \Gamma\left(\frac{3+n}{2}\right)},
$$

but here the singularity at $n \geqq 3$ is not explicit. Thus the mass-overdensity power spectrum can be used to derive the CMB power spectrum as a function of $n, \ell$ and amplitude $Q_{r m s-P S}^{2}$ (Bond \& Efstathiou 1987).

The power spectra $P(k)$ and $\Delta T_{\ell}^{2}$ are close analogs (Appendix L). The scales are determined by $k$ and $\ell$, and the normalization constants are $A$ and $Q_{r m s-P S}$. Equation (4.11) can be taken as the defining equation for $n$ and $Q_{r m s-P S}$, the two parameters of the radiation power spectrum.

For the important $n=1$ Harrison-Zeldovich (HZ) case equation (4.11) reduces to

$$
\Delta T_{\ell}^{2}=\frac{6}{5} \frac{2 \ell+1}{\ell(\ell+1)} Q_{r m s-P S}^{2} \propto \frac{1}{\ell+1} .
$$

Figure 4.1 (bottom) shows a simple example of using equation (4.12) in the Legendre polynomial expansion of the correlation function (equation (4.3)).

If the data set is not sensitive to the quadrupole, one can still obtain $Q_{r m s-P S}$ from the data since it is a normalization constant for all the $\ell$ multipoles. The normalizing amplitude $Q_{r m s-P S}$ is a parameter of the model and is not to be confused with the actual quadrupole $Q_{r m s}$ of our small observable Universe. In Section 10.2 we discuss obtaining $n$ and $Q_{r m s-P S}$ by fitting theoretical correlation functions $C_{n}(\alpha)$ to the observed correlation function $C_{d}(\alpha)$.

A large number of recent articles has dealt with the determination of $n$ and $Q_{r m s-P S}$. All of the methods used suffer from a near degeneracy when fitting for both $n$ and $Q_{r m s-P S}$. Some workers even quote a line in $\left[n, Q_{r m s-P S}\right]$ space rather than a point or an error ellipse (see Figure 10.2). This degeneracy is independent of whether $n$ and $Q_{r m s-P S}$ are determined from the correlation function, the power spectrum or the genus of the maps. Figure 4.2 displays the simple reason for this degeneracy: the effect of increasing $n$ is 
very similar to the effect of increasing $Q_{r m s-P S}$. Thus larger $n$ values require smaller $Q_{r m s-P S}$ values and vice versa. Observations at smaller angular scales will help to break this degeneracy.

\subsection{Cosmic Variance}

Cosmologists are interested in the entire Universe, not just our Hubble volume. The surface of last scattering which we observe is one sample of the entire Universe. Cosmological models do not make predictions about our particular surface of last scattering; they cannot predict where a hot or cold spot will be. They make predictions about an ensemble of observable universes i.e., about the parent distribution. If we are interested in comparing the DMR measurements to cosmological models we have to take cosmic variance into account (see Figure 4.3).

In a popular class of cosmological models, the $a_{\ell m}$ in equation (4.5) are drawn from Gaussian parent distributions with $\ell$-dependent dispersions. There are $2 \ell+1 m$ values for each $\ell$, thus $\Delta T_{\ell}^{2}$ is a $\chi^{2}$ distributed variable of $2 \ell+1$ degrees of freedom. The cosmic variance is $2<\Delta T_{\ell}^{2}>^{2} /(2 \ell+1$ ) (Smoot et al. 1992).

The smaller the $\ell$ value, the more sparse is our sample of the parent distribution and therefore the larger the cosmic variance. The CMB quadrupole is not as accurate a measure of the ensemble quadrupole as the CMB octopole is a measure of the ensemble octopole and so on. We denote the measured quadrupole of our surface of last scattering by $Q_{r m s}$ while $Q_{r m s-P S}$ is based on the better sampled $\ell>2$ terms of the power spectrum and is therefore a measure of the rms quadrupole of the ensemble. Thus even though the quadrupole of the DMR two year data set is $Q_{r m s}=6 \pm 3$ (Bennett et al. 1994) the "COBE normalization", $Q_{r m s-P S}$, is in the range $[17 \mu \mathrm{K}, 20 \mu \mathrm{K}]$ (see Figure 10.2).

\subsection{Beam Smoothing}

Thus far we have ignored the complication of the beam smoothing on $C(\alpha)$. Beam smoothing lowers the amplitude of $C(\alpha)$ for small $\alpha$. If the temperatures of the true sky expanded in real spherical harmonics are (Appendix $H$ )

$$
\delta T(\theta, \phi)=\sum b_{\ell m} F_{\ell m}(\theta, \phi)
$$




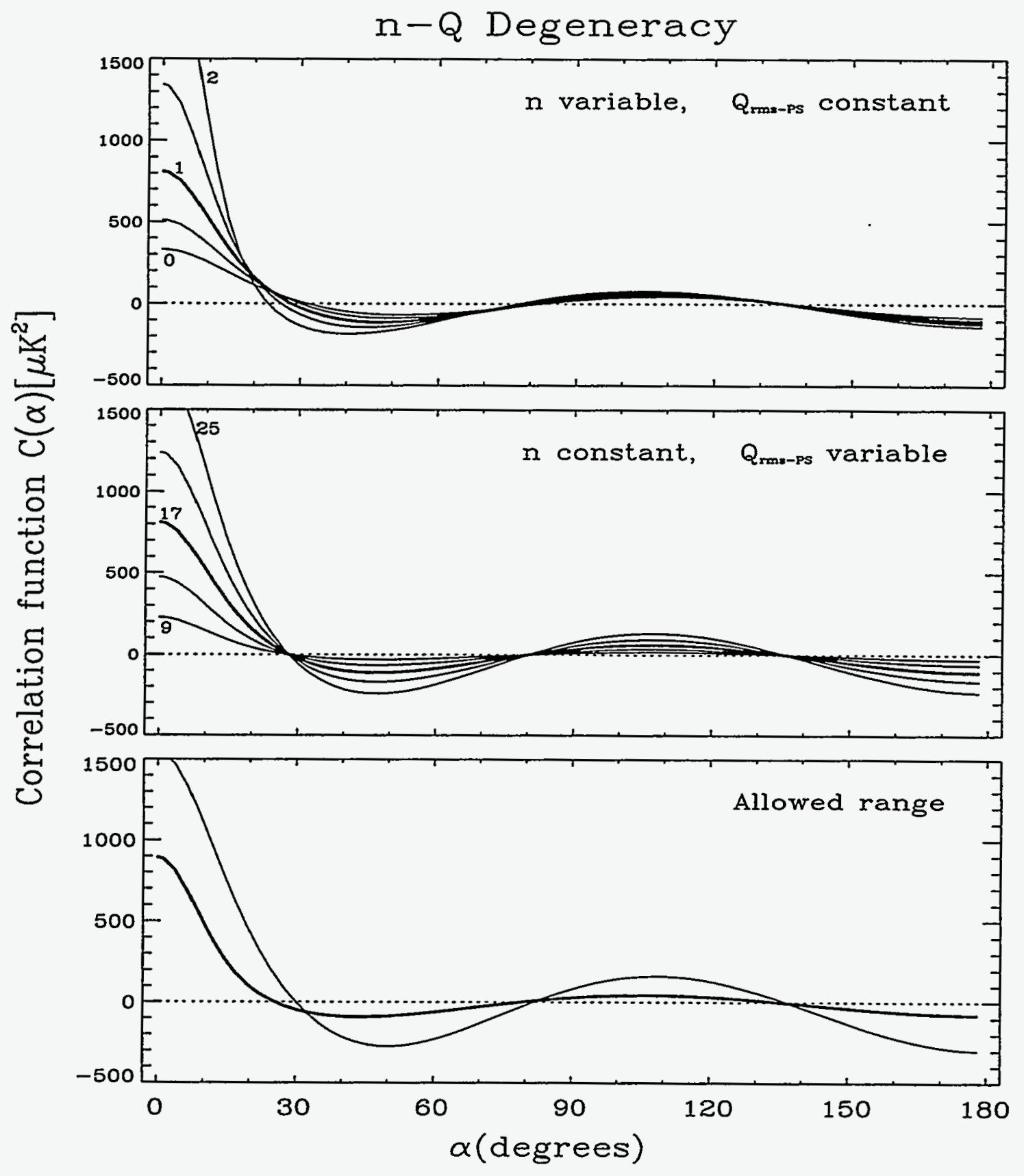

Figure 4.2: Degeneracy of $n$ and $Q_{r m s-P S}$. The effect of varying $n$ in equation (4.11) is very similar to the effect of varying $Q_{\tau m s-P S}$. With $Q_{\tau m s-P S}$ fixed at $17 \mu \mathrm{K}$ (top panel), $C(\alpha)$ for $n=0,0.5,1.0,1.5,2.0$ is shown. The lines of $n=0,1$ and 2 are labeled. With $n$ fixed at 1 (middle panel), $C(\alpha)$ for $Q_{r m s-P S}=9,13,17,21,25$ is shown. The lines of $Q_{r m s-P S}=9,17$ and $25 \mu \mathrm{K}$ are labeled. The two curves in the bottom panel refer to the extreme values allowed by the two year data from the $68 \%$ confidence levels in Figure 2 of Górski et al. (1994): $[\mathrm{n}, \mathrm{Q}]=[1.55,13.5]$ (thick) and $[0.43,30.5]$ (thin). 


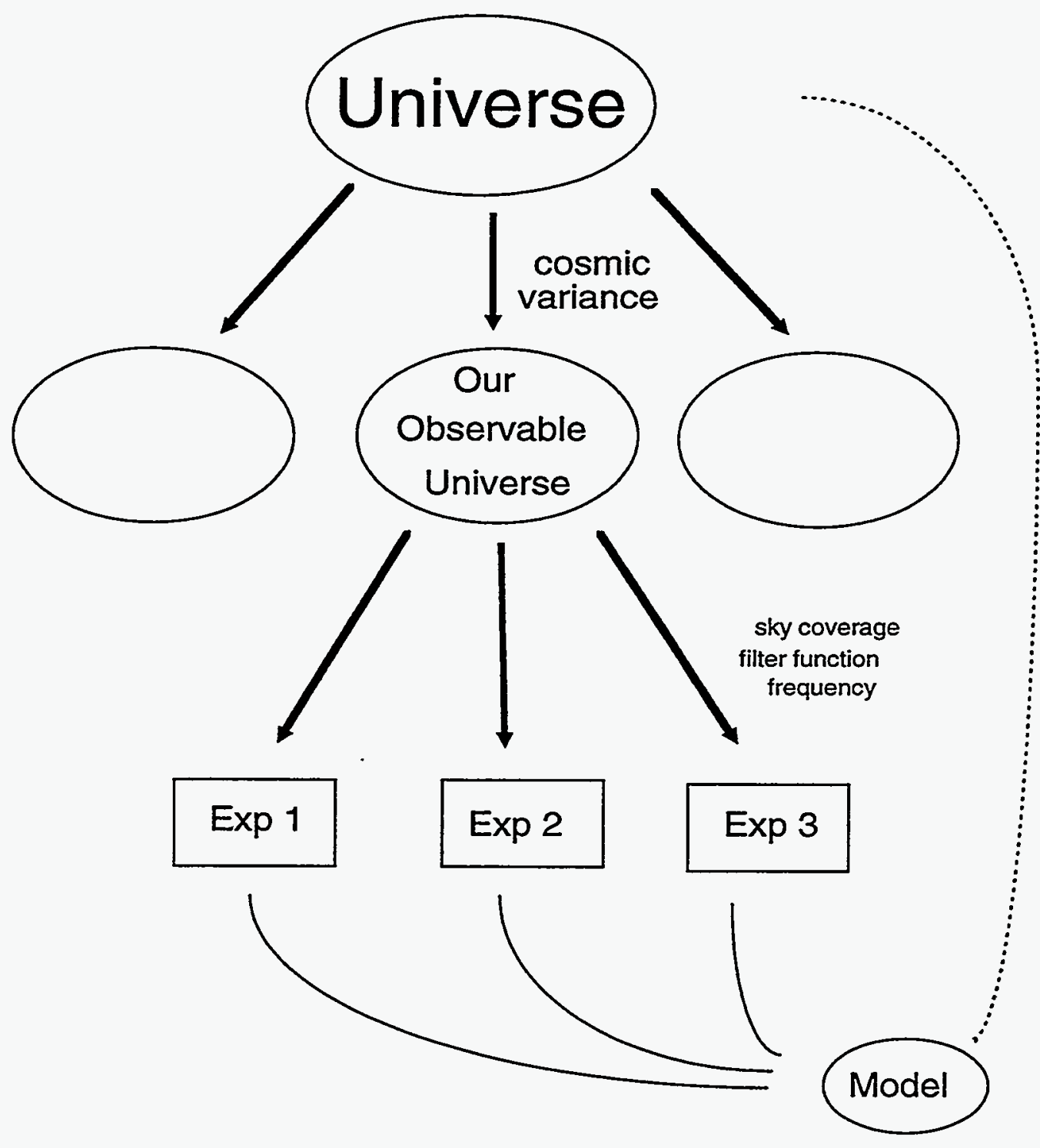

Figure 4.3: Cosmic variance. Cosmological models of the Universe are not specific to our observable Universe. Comparison of cosmological models with experiments necessarily includes cosmic variance. Since all experiments sample the same observable universe, they can be compared with each other without considering cosmic variance. 
then the beam-smoothed sky is

$$
\delta T_{b}(\theta, \phi)=\sum b_{\ell m} W_{\ell} F_{\ell m}(\theta, \phi)
$$

where $W_{\ell}$ are the Legendre polynomial expansion coefficients of the DMR beam gains (Wright et al. 1994a);

$$
\begin{aligned}
\operatorname{Gain}(\theta) & =\sum_{\ell}\left(\frac{2 \ell+1}{2}\right) W_{\ell} P_{\ell}(\cos \theta) \\
W_{\ell} & =\int_{-1}^{1} \operatorname{Gain}(\theta) P_{\ell}(\cos \theta) d \cos \theta .
\end{aligned}
$$

The correlation function including the effect of beam smoothing is

$$
C(\alpha)=\sum_{\ell=2}^{\ell_{\max }} \Delta T_{\ell}^{2} W_{\ell}^{2} P_{\ell}(\cos \alpha),
$$

where $W_{\ell}^{2}$ is the window function which accounts for the beam smoothing. For Gaussian beams $W_{\ell}^{2}=e^{-\ell(\ell+1) / \ell_{b}^{2}}$ where $\ell_{b}$ cuts off the small angular scale power for $\ell \gtrsim \ell_{b}$ because the beam averages over those scales (Wilson \& Silk 1981). This cutoff is related to the FWHM and the beam dispersion $\sigma_{b}$ by

$$
\ell_{b} \approx \frac{180^{\circ}}{\pi} \frac{\sqrt{8 \ln 2}}{F W H M}=\frac{1}{\sigma_{b}} .
$$

where FWHM is in degrees and $\sigma_{b}$ is in radians. A somewhat more precise formula is given by Bond (1994),

$$
\ell_{b}+\frac{1}{2}=\frac{1}{2 \sin \left(\sigma_{b} / 2\right)}
$$

When combined with the smearing due to the 0.5 second integration time, the $2.6^{\circ}$ pixelization and the $2.6^{\circ}$ correlation function binning, the effective DMR beam is well approximated by a $\sigma_{b}=3.2^{\circ}$ or $\ell_{b}=17.9$ (Smoot et al. 1992). The Smoot et al. (1992) correlation function results used a Gaussian approximation to the beam and included corrections for the $1.3^{\circ}$ integration time, $2.6^{\circ}$ pixelization and the $2.6^{\circ} C(\alpha)$ binning. More careful beam patterns were developed by Wright et al. (1993) and Kneissl \& Smoot (1993). We use the latter in our analysis.

The importance of the beam and the contribution of the high $\ell$ terms of the power spectrum is attested to by the results of Wright et al. (1994b) who report best fit $n$ values of $n=1.46$ for $3 \leq \ell \leq 19$ and $n=1.25$ for the larger range $3 \leq \ell \leq 30$. This implies that in the top panel of Figure 4.4 the power in the thick dotted line in the $\ell>19$ region can change the value of $n$ substantially. We typically run simulations out to $\ell=39$ to include the effects of high $\ell$ terms. 

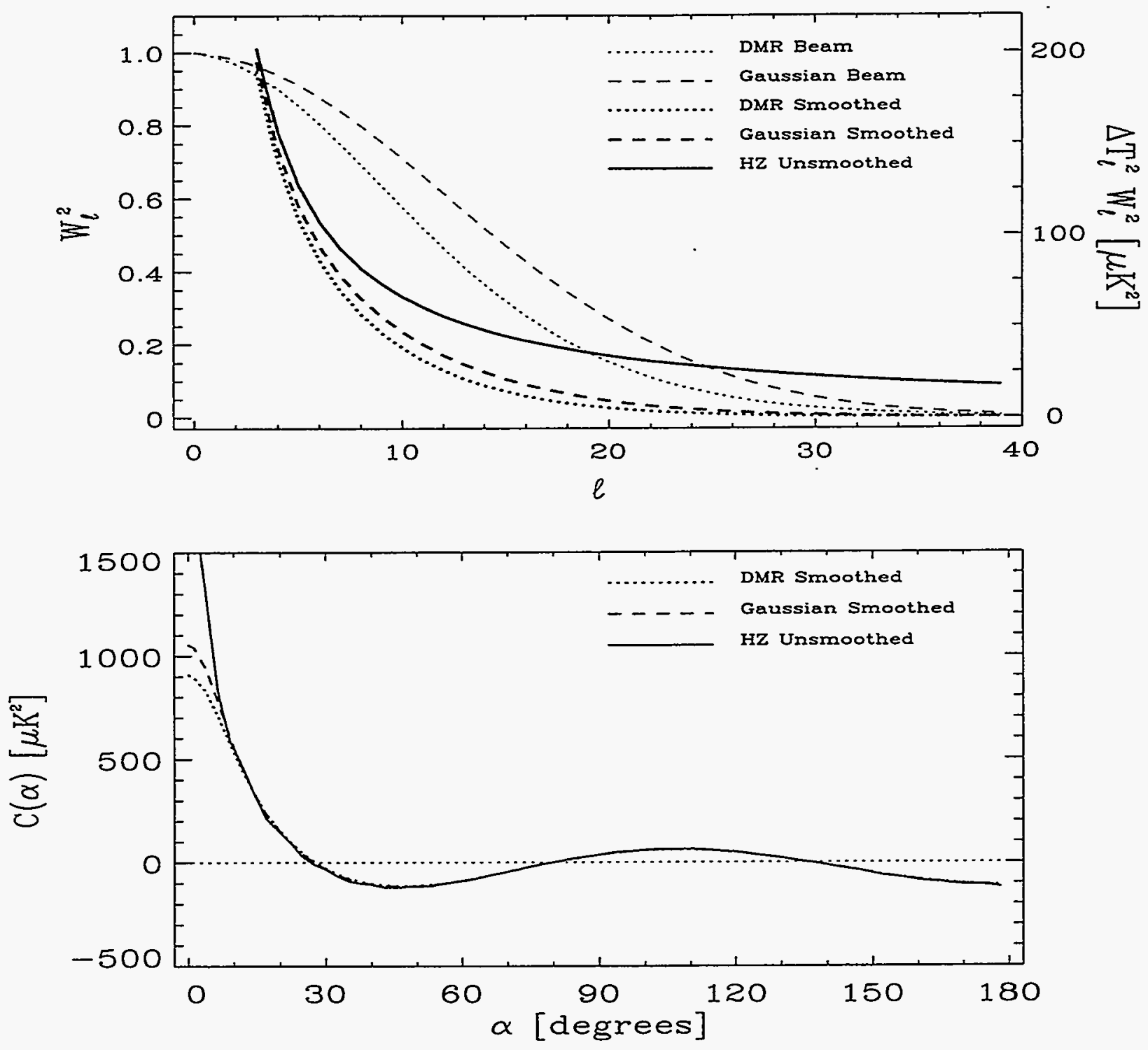

Figure 4.4: Beam Smoothing of Power and the Correlation Function. The solid line in the top panel is the power spectrum $\Delta T_{\ell}^{2}\left(n=1, Q_{r m s-P S}=17 \mu \mathrm{K}\right)$. When convolved with the DMR window function (thin dotted), it becomes the thick dotted line. When convolved with the Gaussian approximation to the DMR beam (thin dashed), it becomes the thick dashed line. The unsmoothed HZ power spectrum and the two beam-convolved power spectra were used to produce the correlation functions of the same line style in the bottom panel. $\mathrm{C}\left(\alpha<10^{\circ}\right)$ is dramatically changed. Notice the $150 \mu \mathrm{K}^{2}$ lower $\mathrm{C}(0)$ value for the actual beam pattern when compared to the Gaussian approximation. The Gaussian beam uses $\ell_{b}=17.9\left(\sigma_{b}=3.2^{\circ}\right)$ while the "DMR beam" is essentially the $G_{\ell}$ 's from Wright et al. (1994a) with small corrections (see Kneissl \& Smoot 1993). The correlation functions have had the best fit mean, dipole and quadrupole removed from the maps. 


\subsection{Correlation Function from DMR Maps}

The two-point auto-correlation function of a pixelized DMR map is the weighted average product of temperatures separated by angle $\alpha$

$$
C(\alpha)=\frac{1}{W} \sum_{i, j} w_{i} w_{j} T_{o b s, i} T_{o b s, j}
$$

where the sum is over all sky pixel pairs $i j$ whose separation angle $\alpha_{i j}$ lies within half a bin width of $\alpha$. We use the weights $w_{i}=1 / \operatorname{var}\left(T_{o b s, i}\right)$ and we approximate $\operatorname{var}\left(T_{o b s, i}\right)$ by $\sigma_{c h}^{2} / N_{i}$, where $\sigma_{c h}^{2}$ is the channel specific pixel noise variance per observation. The normalization factor is $W=\sum_{i, j} w_{i} w_{j}$. Setting $w_{i}=w_{j}=1$ yields an unweighted correlation function. For cross-correlation functions, $i$ refers to one map and $j$ to the other. To avoid Galactic contamination, we usually exclude from the sum all pixels close to the Galactic plane.

We remove best fit means, dipoles and optionally quadrupoles from the maps before the correlation function is accumulated. We are interested in anisotropies around the mean level so we do not consider the monopole $\ell=0$ term. We subtract the best-fit dipole $(\ell=1)$ since it is dominated by the kinetic dipole. The best fit quadrupole is sometimes removed, thus $\ell_{\min }=2$ or 3 .

Figure 4.5 shows the correlation function of the actual dipole in the DMR maps as well as the influence of the Galaxy. For small $\alpha$, the correlation function of the dipole is three orders of magnitude above the signal detected. The effect of the Galaxy is two orders of magnitude above the signal so these effects must be handled carefully.

\subsection{RMS Temperature Fluctuations from $C(\alpha)$}

The rms temperature is one of the most important statistics of the DMR maps and it is a subset of the correlation function. The auto-correlation function at zero lag is the rms temperature fluctuation in the map. The weighted auto-correlation function is

$$
C_{w}(0)=\frac{\sum_{i} N_{i}^{2} T_{o b s, i}^{2}}{\sum_{i} N_{i}^{2}}=\sigma_{w}^{2}
$$

and the unweighted version is

$$
C_{u}(0)=\frac{\sum_{i} T_{o b s, i}^{2}}{\sum_{i} 1}=\sigma_{u}^{2} .
$$

Thus the sky signal can be extracted from auto-correlations of the $(A+B) / 2$ and $(A-B) / 2$ maps (equation 4.24) or even more simply from the cross-correlation function of map A 


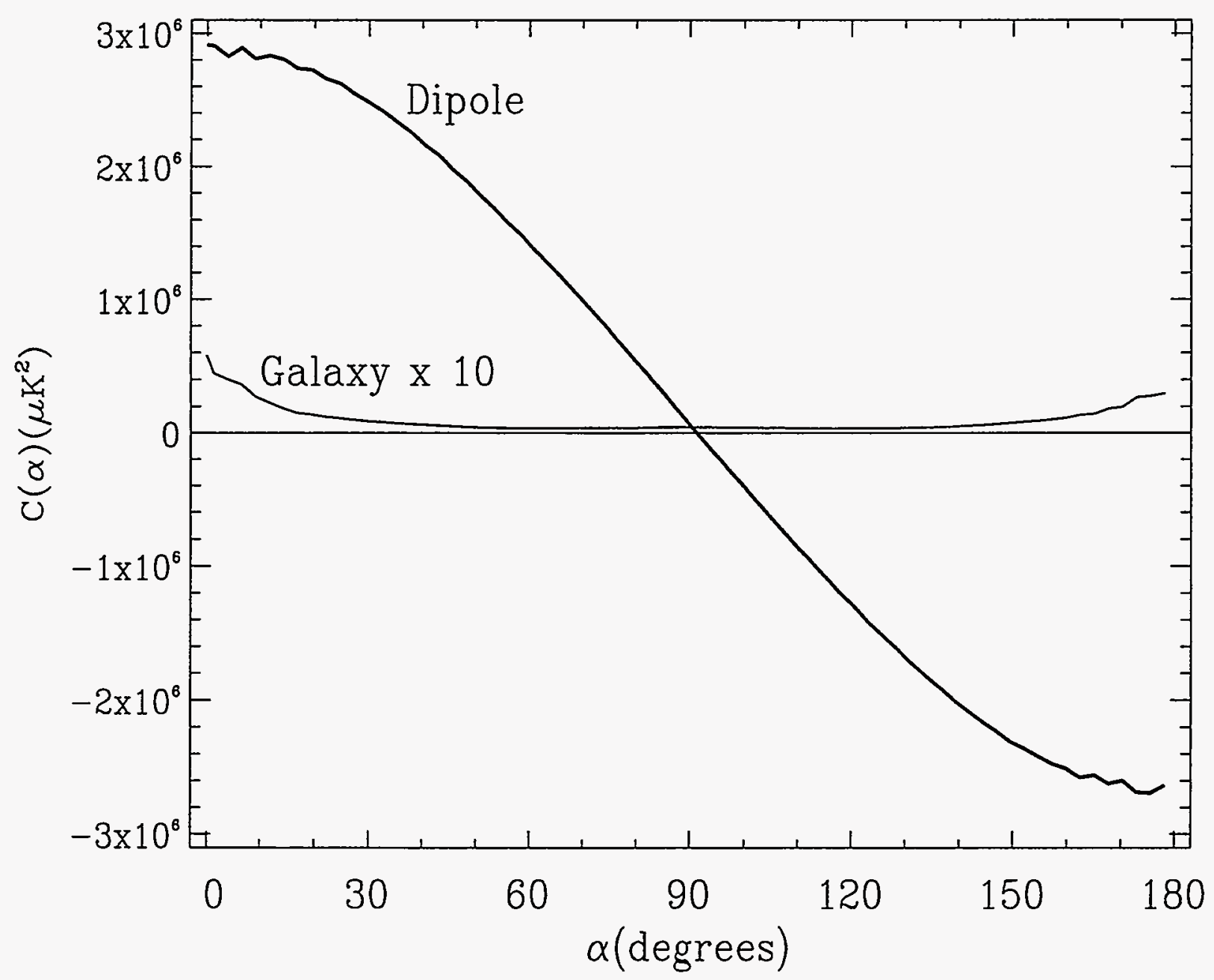

Figure 4.5: Correlation Functions of Dipole and Galaxy. The dipole (thick) is a pure $\ell=1$ of amplitude $\sim 3000 \mu \mathrm{K}^{2}$. After removing the best fit dipole in the Galactic latitude $|b|>20^{\circ}$ region but keeping the Galactic plane during the computation of the correlation function, one obtains (thin) a Galaxy dominated curve which is dominated by a quadrupole but has other structure. The correlation function of the Galaxy has been multiplied by a factor of 10 to make its shape visible on this scale. 
with $B$ (equation (4.25)

$$
\begin{aligned}
\sigma_{s k y}^{2} & =\sigma_{A+B}^{2}-\sigma_{A-B}^{2} \\
\sigma_{s k y}^{2} & =C_{A+B}(0)-C_{A-B}(0) \\
\sigma_{s k y}^{2} & =C_{A x B}(0)
\end{aligned}
$$

where $\sigma_{s k y}^{2}$ can be either from weighted or unweighted correlation functions. The last two equations follow from equation (4.20) when we express the observed map temperatures as the sum of signal plus noise: $T_{o b s, i}=T_{i}+n_{i}$ in map A and $T_{o b s, j}=T_{j}+n_{j}$ in map $\mathrm{B}$. We have used the fact that all the cross products have zero expectation values except the signal $T_{i} T_{j}$. If only one map is available and the noise values in neighboring pixels are nearly independent, a good estimate of the temperature fiuctuations $\sigma_{s k y}^{2}$ can be obtained from the first non-zero bin of the correlation function. Smoothing the maps before calculating $C(\alpha)$ would destroy this nice property by spreading the noise into the neighboring pixels.

The relative merits of the various weightings of $\mathrm{rms}$ statistics $\left(\sum_{i} T_{i}^{2}, \sum_{i} w_{i} T_{i}^{2}\right.$, $\sum_{i} w_{i}^{2} T_{i}^{2}$ or for two maps $\sum_{i=j} w_{i} w_{j} T_{i} T_{j}$ ) will not be discussed here except for the remark that the square root of the weighted auto-correlation function at zero lag $\left(\sum_{i} w_{i}^{2} T_{i}^{2}\right)$ minimizes the variance due to the noise in the map pixel temperatures while unit weighting minimizes the effect of cosmic variance (see Smoot et al. 1994, Bennett et al. 1994, Banday et al. 1994).

Because of the importance of $C(0)$, Monte Carlo simulations were performed to monitor the behaviour of $C\left(\alpha \lesssim 15^{\circ}\right)$ as a function of bin choice. The pixel size bin of $2.6^{\circ}$ minimized oscillations of the output $C\left(\alpha \lesssim 15^{\circ}\right)$ around the known input. Thus we chose $2.6^{\circ}$ to bin the correlation function and it is the standard used in this thesis.

\subsection{New Way to Estimate $Q_{r m s}$}

We routinely subtract the mean and dipole (md) or the mean, dipole and quadrupole (mdq) from each map before computing the auto or cross-correlation function

$$
\begin{aligned}
C_{m d}(0) & =\sum_{2}^{\ell_{\max }} \Delta T_{\ell}^{2} W_{\ell}^{2} \\
C_{m d q}(0) & =\sum_{3}^{\ell_{\max }} \Delta T_{\ell}^{2} W_{\ell}^{2} \\
C_{m d}(0)-C_{m d q}(0) & =\Delta T_{2}^{2} W_{2}^{2} \equiv Q_{r m s}^{2} W_{2}^{2} .
\end{aligned}
$$


We have $W_{2}=0.9855$ (Wright et al. 1994a) thus the beam smoothing requires a $1.5 \%$ correction:

$$
Q_{r m s}=1.015 \sqrt{C_{m d}(0)-C_{m d q}(0)}
$$

In equation (4.29) the correlation functions can be either auto or cross-correlation functions. We can also use unweighted or weighted correlation functions. For cross-correlations, the $Q_{\text {rms }}$ solved for is not associated with an individual map. It is a "cross" quadrupole, i.e., the actual sky quadrupole which is shared by the two maps which went into the crosscorrelation functions. Table 7.3 are the results for $Q_{\tau m s}$ computed from equation (4.29) with both weighted and unweighted cross-correlation functions.

\subsection{Bias Due to Multipole Subtraction and Galactic Latitude Cuts}

It is often the case that best-fit multipoles are removed from the map before the analysis. The DMR is not sensitive to the mean level of the CMB sky (Appendix J), so it is reasonable to remove the mean in the region in which $C(\alpha)$ is computed. The kinetic dipole is so large $(\sim 3363 \mu \mathrm{K})$ and inseparable from any possible intrinsic dipole that we subtract it out before the analysis. We also often remove the best-fit quadrupole because any residual Galactic foreground will be primarily quadrupolar and the biggest term in the systematic error budget is the quadrupolar pattern of the magnetic susceptibility errors. Removal of the mean and dipole (and quadrupole) after the Galactic cut removes some of the $\ell=3$ and $\ell=4$ power of the true sky power as well as the model input. The Galaxy is a strong source of foreground emission (Figure 4.5) which must be removed or corrected. Correcting for the Galaxy is a difficult task and is the subject of Bennett et al. (1992a). Typically we make a straight Galactic latitude cut and remove all pixels with $|b|<20^{\circ}$ leaving 4016 pixels or $2 / 3$ of the sky.

To investigate the possible biases in the correlation function introduced by the multipole subtractions and Galactic cut we have made noise simulations of $53 \mathrm{~A}$ and $53 \mathrm{~B}$ and

applied the two most common multipole subtraction options: mean+dipole (md) and mean+dipole+quadrupole (mdq) for $0^{\circ}$ and $20^{\circ}$ Galactic cuts (Figure 4.6). We find that cross-correlations are immune to any bias from either the multipole subtraction or the Galactic latitude cut when no sigal is present. Any bias in the cross-correlation is less than $10 \%$ of the $68 \%$ noise confidence level in that bin. This result is true for both $0^{\circ}$ and $20^{\circ}$ 
Galactic cuts.

The auto-correlations suffer from a bias. In Figure 4.6 both multipole subtraction options are plotted for a $20^{\circ}$ Galactic latitude cut on $53 \mathrm{~A}$ noise simulations. The range of bias is seen to be $\sim 20$ to $\sim 70 \mu \mathrm{K}^{2}$ and the bias in small $\alpha$ bins is the largest. Comparisons with the 53B simulations indicate that $\frac{\text { bias }_{C_{A}(\alpha)}}{\operatorname{bias}_{C_{B}(\alpha)}} \approx \frac{\sigma_{A}^{2}}{\sigma_{B}^{2}}$ where $\sigma_{A}^{2}$ and $\sigma_{B}^{2}$ are the noise variances per observation in maps $53 \mathrm{~A}$ and $53 \mathrm{~B}$ respectively. Thus these biases scale as the noise variance in the map $\sigma_{c h}^{2}$.

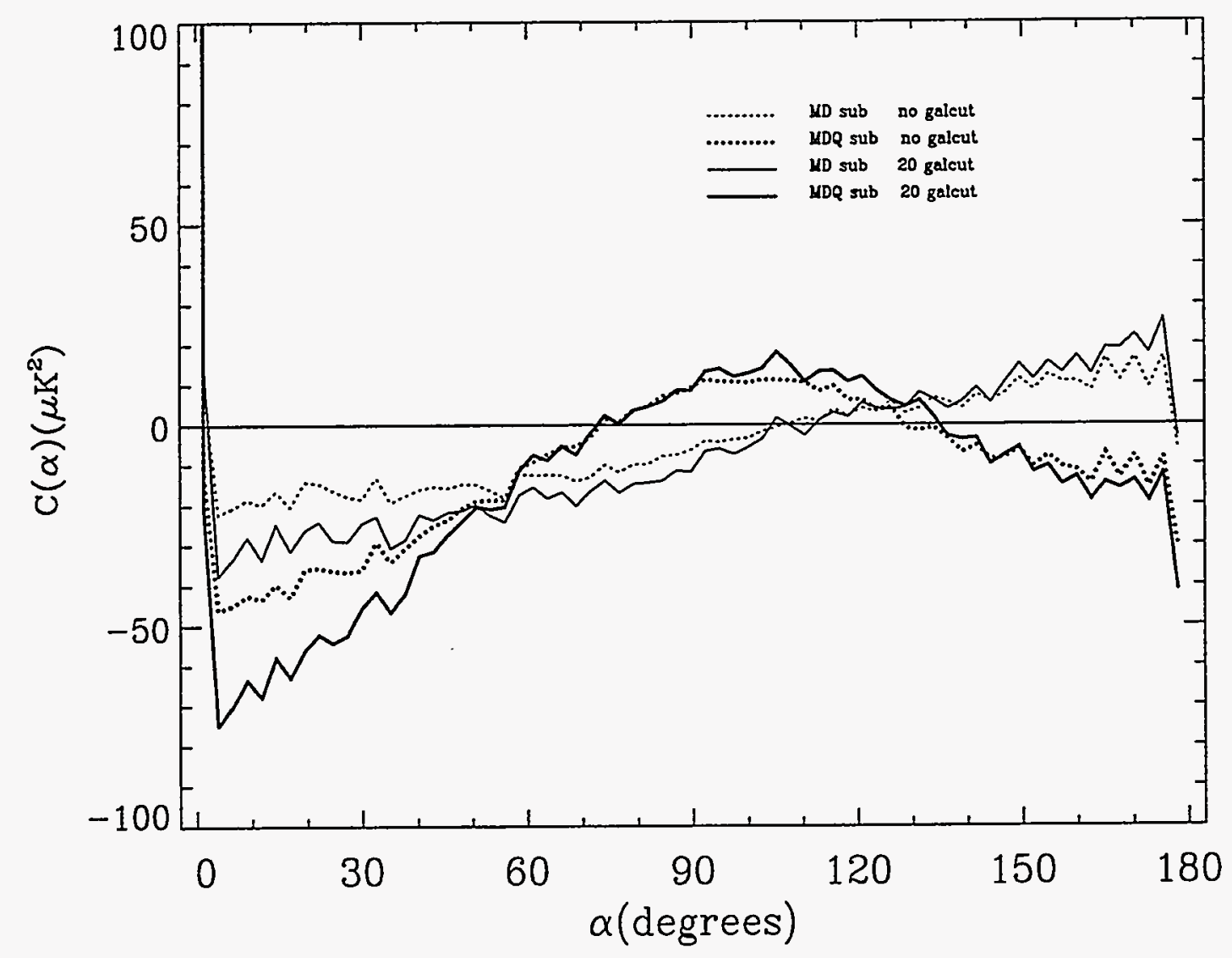

Figure 4.6: Auto-correlation Multipole Subtraction Bias. Comparison of $20^{\circ}$ Galactic plane cut with no cut. Comparison of removal of best fit mean and dipole (md) with the removal of the best fit mean, dipole and quadrupole (mdq). Weighted correlation functions are plotted and unweighted multipole fits have been subtracted as indicated. Mean of 1500 simulations of year one 53A with noise variance $(24.9 \mathrm{mK})^{2}$ per observation. 


\title{
Chapter 5
}

\section{The First Year Results}

\begin{abstract}
We present a detailed correlation function analysis of the first year data set which contributed significantly to the DMR discovery of anisotropy and was partially reported in Smoot et al. (1992). We use the correlation function to help determine that the observed structure cannot be attributed to noise or Galactic foreground. As expected of a CMB signal, the correlation function signal is consistent with no frequency dependence and no Galactic latitude dependence. The correlation function of the first year data shows statistically significant $(>7 \sigma)$ structure. The power spectral index $n$ obtained from fits to the correlation function is consistent with the Harrison-Zeldovich value. The dipole results from the first year maps are summarized and interpreted.
\end{abstract}

\section{$5.1 \quad$ Introduction}

The COBE collaboration reported the detection of anisotropies in the cosmic microwave background radiation (Smoot et al.1992, Bennett et al.1992b, Wright et al.1992, Kogut et al. 1992). These anisotropies cannot be attributed to systematic errors (Kogut et al. 1992) or any known Galactic foreground (Bennett et al. 1992b).

Smoot et al. (1992) and Wright et al. (1992) presented preliminary correlation function results from the COBE DMR first year skymaps. Since then, the correlation function has become an important standard tool for comparing data to models (Adams et al. 1992, Scaramella \& Vittorio 1993, Seljak \& Bertschinger 1993, Bennett et al. 1994) and data 
to data (Ganga et al.1993). We provide a more complete presentation of the correlation function analysis of the first year DMR sky maps than was presented in Smoot et al. 1992.

In Section 5.2 we describe the ways the correlation function is used to determine the noise level and identify a signal. We present separate channel as well as $A+B$ vs $A-B$ comparisons. In Section 5.3 we establish the Galactic latitude independence of the signal and in Section 5.4 we present our best $53 \times 90$ cross-correlation function, the statistical significance of the signal and the rms temperature fluctuations. In Section 5.5 we briefly summarize and interpret the dipole results.

The data analyzed in this chapter are from the first year "pass 1" processing. It differs from the first year released data set only in that the Galactic coordinate system was used rather than the ecliptic coordinate system. The "pass 1 " data set is described and compared with the "pass 2" data set in Appendix E.

\subsection{Is It Noise?}

The first year of DMR observations produces the correlation function data sets in Figure 5.1 for the separate channel auto-correlations and Figure 5.2 for the $A+B$ and $A-B$ auto-correlations. The relative noise levels of the six channels are easily seen: the $53 \mathrm{GHz}$ channels have the least noise while the $31 \mathrm{GHz}$ channels have the most. In Figure 5.2 the most sensitive $53 \mathrm{GH} z$ plots display a marked difference between the $A+B$ and $A-B$ versions. The signal ( the positive correlation for $0<\alpha \lesssim 20$ ) stands out clearly above the A-B noise. The $31 \mathrm{GHz}$ and $90 \mathrm{GHz} \mathrm{A}+\mathrm{B}$ correlation functions are consistent with the signal defined by the $53 \mathrm{GHz}$ channels. The individual channel correlation functions are also consistent with the signal however the $31 \mathrm{GHz}$ channels are very noisy and some of its signal is emission from the Galaxy. The $90 \mathrm{~A}$ channel is anomalous in that there is no signal except in the first non-zero bin and this first bin signal is much larger than one expects based on the other channels. Curiously, 90A-B indicates that $90 \mathrm{~A}$ and $90 \mathrm{~B}$ are anti-correlated in the first bin. The second year 90A is not as anomalous (see Figure 8.1) and the anti-correlation between $90 \mathrm{~A}$ and $90 \mathrm{~B}$ is not noteworthy in the Yr2 data (Figure 8.2).

All curves in Figure 5.1 and 5.2 are auto-correlations. The zeroth bin of autocorrelations contains the noise squared and is not shown. Cross-correlations do not have this problem because of the independent pixel noise in the two maps being cross-correlated. 
Separate Channels 1990 Pass1 vs 1990 Pass1 Ecl Channel A

Channel $\mathrm{B}$
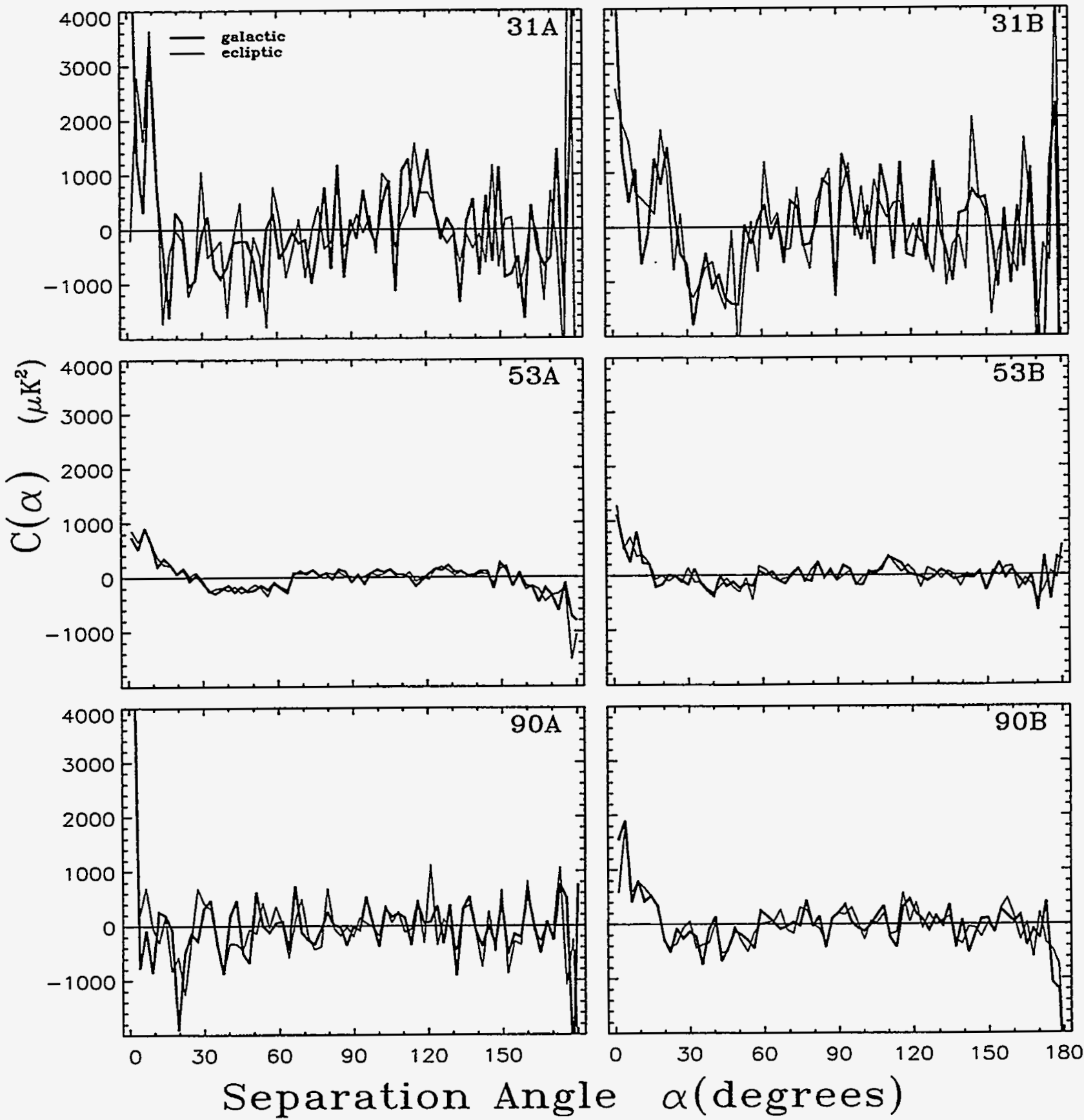

Figure 5.1: Yr1 Separate Channels in Galactic and Ecliptic Coordinates. These are weighted correlation functions for $|b|>20^{\circ}$ and have had the best-fit means, dipoles and quadrupoles removed from each map. 
Auto-Correlations 1990 Pass 1 vs 1990 Pass 1 Ecl

$A+B$
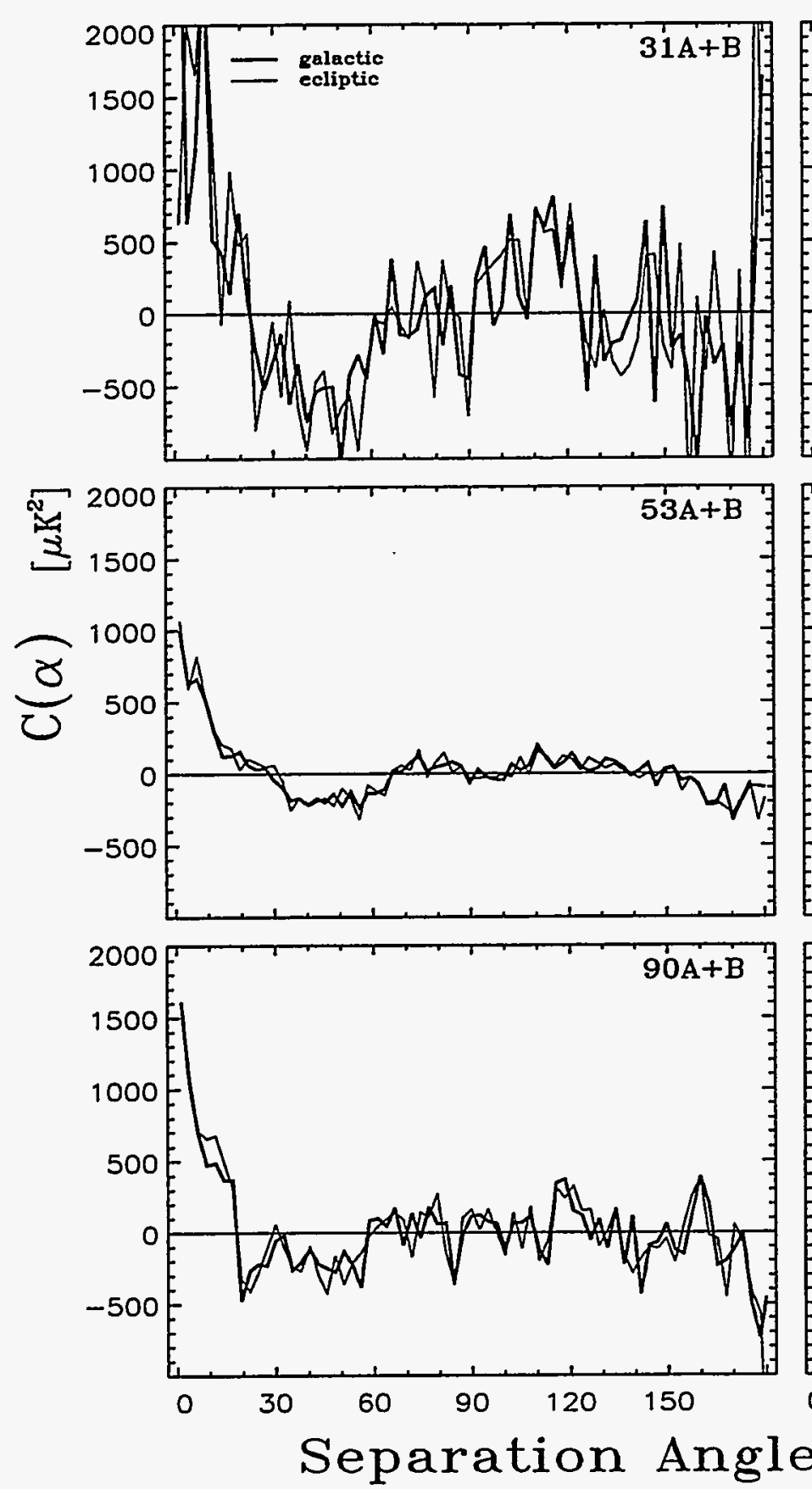

A - B
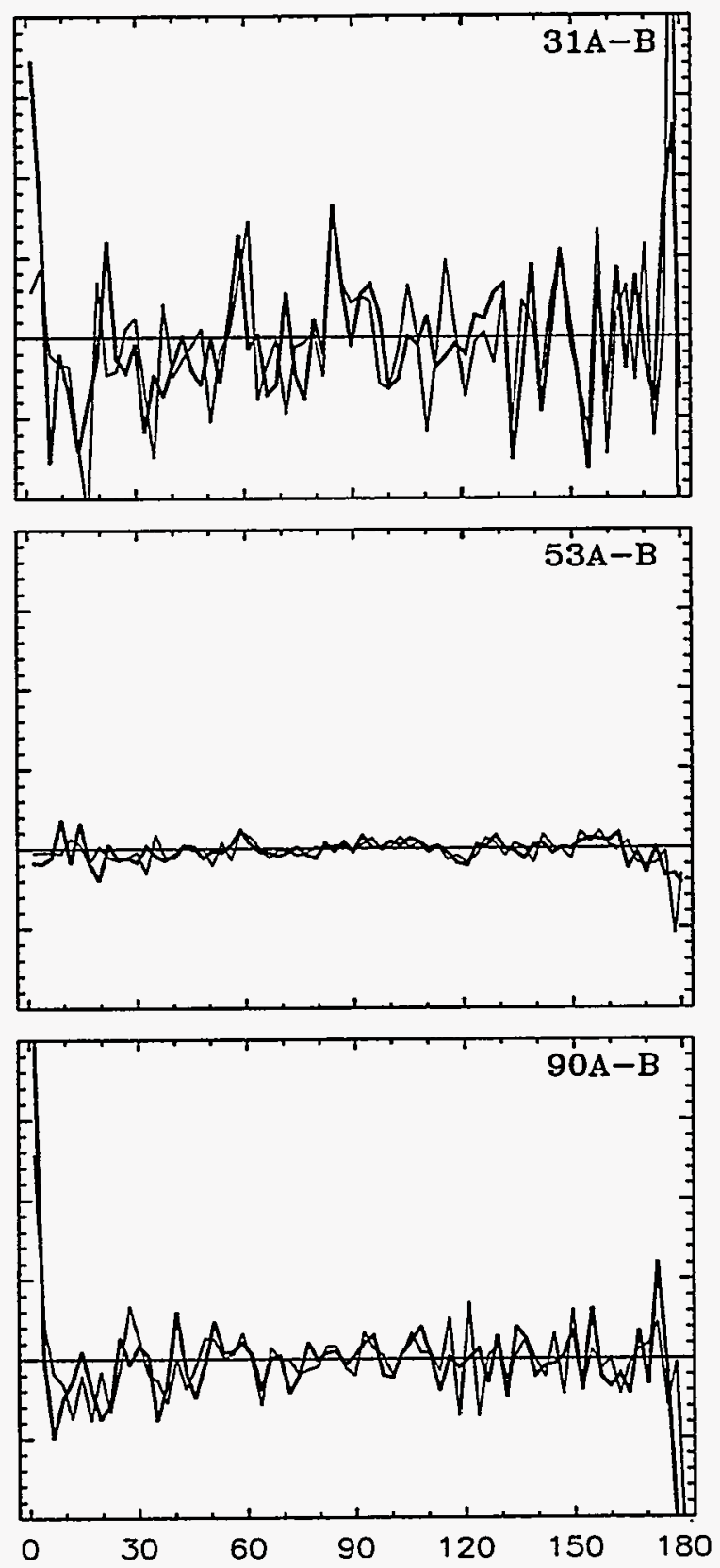

$\alpha$ [degrees]

Figure 5.2: $\mathrm{Yr} 1 \mathrm{~A}+\mathrm{B}$ vs $\mathrm{A}-\mathrm{B}$ comparison in Galactic and ecliptic coordinates. These are weighted correlation functions for $|b|>20^{\circ}$ and have had the best-fit mean, dipole and quadrupole removed from each map. 
In Figures 5.1 and 5.2 both traces for each channel are from the same DMR data however the pixelization grid used to bin the data has been oriented in two ways: using Galactic and ecliptic coordinate systems. The first-year released DMR maps have been pixelized with the ecliptic grid however most DMR analysis has been done with the Galactic maps. The amount of variation seen between the two grids is the same as one gets when one shifts the bin centers by about half a bin when calculating the correlation function.

The region within $\pm 20^{\circ}$ of the ecliptic plane seems to contain most of the visible structure in the DMR maps. This region is also the most undersampled part of the maps due to the DMR observation strategy and Moon cuts (compare Figures 2.3 \& 1.4). The ratio of the number of observations in highly sampled regions to the number in sparsely sampled regions is $\approx 5 / 1$ (Table E.1). Concerned that a disproportionately large amount of the signal seen in the correlation function might be coming from this region, we calculated $C(\alpha)$ for ecliptic plane cuts of $10^{\circ}, 20^{\circ}, 30^{\circ}$ and $40^{\circ}$ and found that this was not the case.

\subsection{Is It Galaxy?}

The source of the signal could be Galactic foreground (Bennett et al.1992b). There are simple but powerful.ways to use the correlation function to determine the extent to which Galactic foreground is causing the signal. One is the frequency dependence of the signal and the other is the Galactic latitude dependence. The frequency independence of CMB anisotropy distinguishes it from the three major Galactic foregrounds (see Section 2.3). Thus a comparison of the signal seen in the plots of $C(\alpha)$ for the three frequencies can characterize the spectrum of the source. All three A+B curves of Figure 5.2 are consistent with the signal as defined by the $53 \mathrm{~A}+\mathrm{B}$ correlation function (see Table 7.1).

If the observed signal were produced by some combination of synchrotron, dust and freefree emission from our Galaxy we would expect to find that the signal would vary with the frequency of the radiation and would depend strongly on the Galactic latitude cut applied (see Section 7.2). Figures 5.3 and 5.4 show Galactic latitude cuts $10^{\circ}, 20^{\circ}, 30^{\circ}$ and $40^{\circ}$ for the auto-correlation of $53 \mathrm{~A}+\mathrm{B}$ and the cross-correlation of $53 \mathrm{~A}+\mathrm{B}$ with $90 \mathrm{~A}+\mathrm{B}$. The similarity of the $20^{\circ}, 30^{\circ}$ and $40^{\circ}$ galactic in the $\alpha<20^{\circ}$ region is evidence for the Galactic independence of the signal.

Besides the fact that Figure 5.3 shows auto-correlations while Figure 5.4 shows crosscorrelations there is a procedural point worth mentioning. For Figure 5.3 the subtracted 


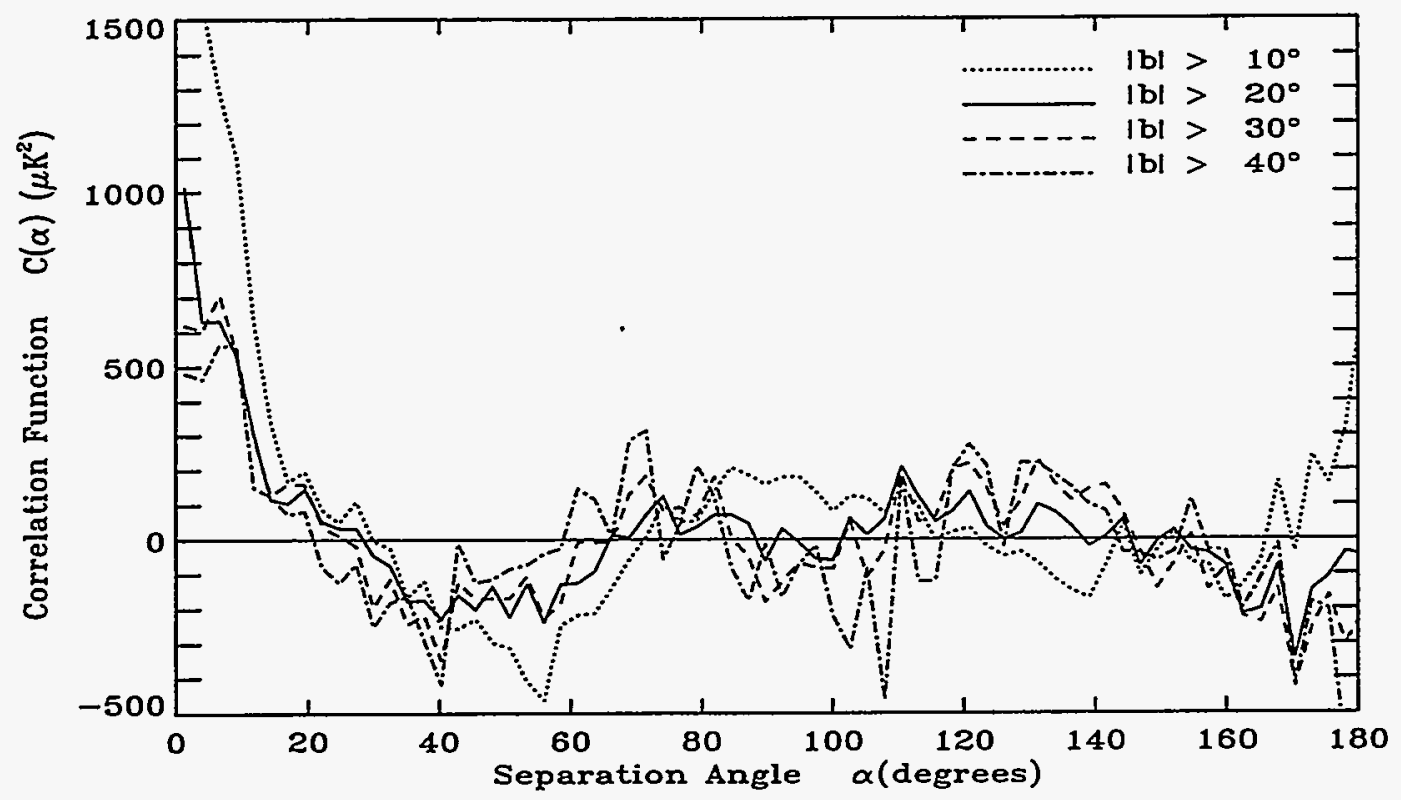

Figure 5.3: Yr1 Galactic Latitude Cut Independence of the $53 \mathrm{GHz} \mathrm{A}+\mathrm{B}$ auto-correlations for cuts $|b|>20^{\circ}$. For each cut, the best-fit mean, dipole and quadrupole to the region outside the cut was removed (Figure 2 of Smoot et al. 1992).

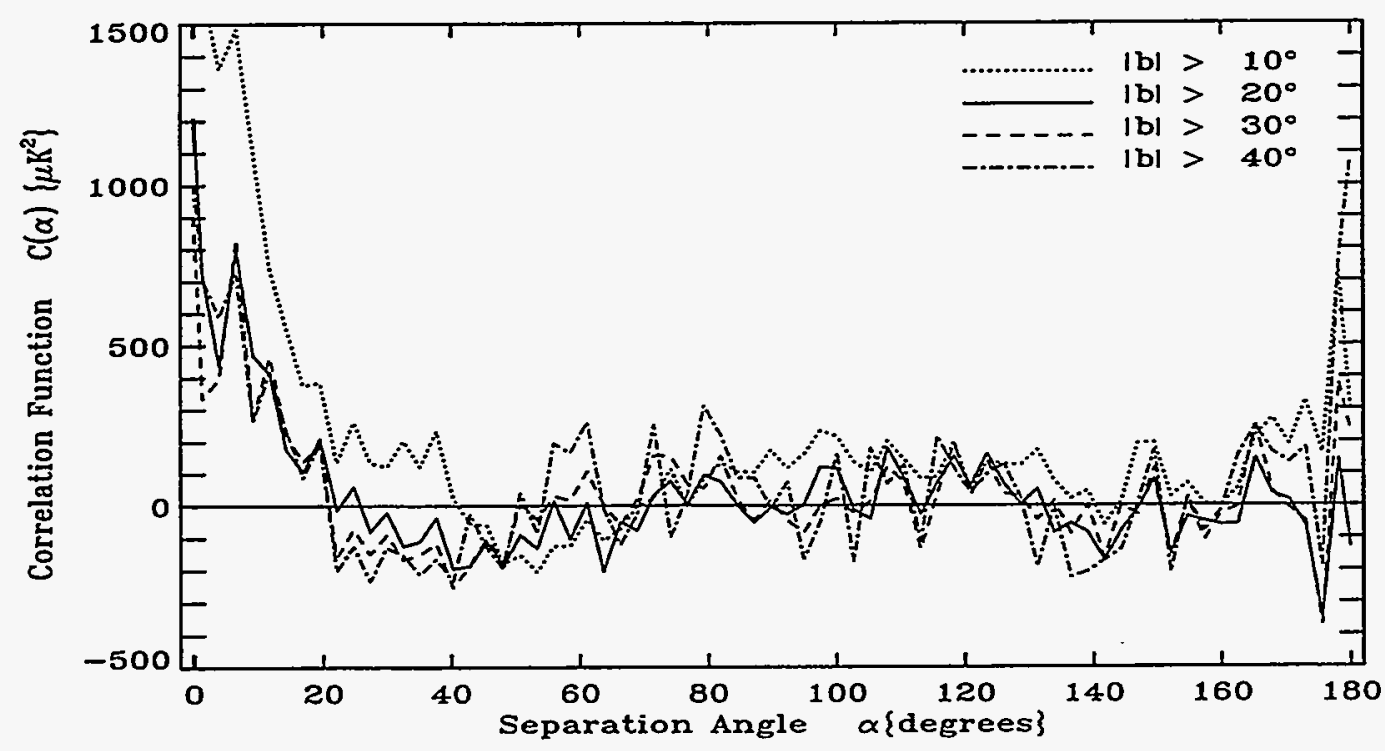

Figure 5.4: Yr1 Galactic Latitude Cut Independence. Cross-correlations of Yr1 A+B 53 $\mathrm{GHz}$ with $90 \mathrm{GHz}$. Notice that the signal is very stable for $20^{\circ}, 30^{\circ}$ and $40^{\circ}$ cuts. 
best fit mean, dipole and quadrupole were fit to the region $|b|>$ galcut for the four galcuts shown while for Figure 5.4 the subtracted best fit mean, dipole and quadrupole were fit to the region $|b|>20^{\circ}$ independent of the galcut. The signal is slightly more robust to Galactic latitude cuts when this more correct procedure is used.

Based on Figures 5.1 and 5.2 one can conclude that there is a signal in the first year DMR data. It does not have the frequency dependence or spatial dependence of any known Galactic foreground (Figures 5.3 and 5.4). Thus it is consistent with the expected frequency independence of CMB anisotropies which should show no particular fondness for the Galactic plane. The source of the signal could be systematic errors, correlated noise, or extra-galactic sources such as a hot inter-galactic medium. Systematic errors are discussed in detail in Kogut et al. (1992) and Bennett et al. (1994). Correlated noise is the subject of Lineweaver et al.(1994b) (see Chapter 9 for the unabridged version of this work). Galactic foreground is treated in Bennett et al. (1992) while known extra-galactic sources are found not to be causing the signal (Bennett et al.1993). We conclude that the most economical explanation is that the signal is CMB. This conclusion is reinforced by the correlation function analysis of the $2 \mathrm{Yr}_{\mathrm{I}}$ data set (Chapter 7) and comparison of the $\mathrm{Y}_{\mathrm{r}} 1$ with the $\mathrm{Yr} 2$ (Chapter 8).

\subsection{If It's CMB, How Large is the Signal?}

One can obtain a measure of the statistical significance of the correlation function signal by computing the $\chi^{2}$ of $C(\alpha)$ (equation (8.1)). The statistical significance is then $\sqrt{\chi^{2}-D O F}$ as described in Section 8.3. Using the best first year cross-correlation of 53

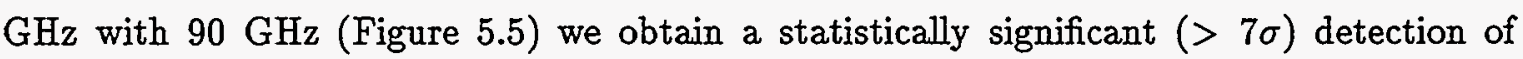
structure. The "conservative" error bars used to obtain this result are described in Section 6.2. The first zero-crossing of $C(\alpha)$ is the characteristic size of the hot and cool spots in the map. The grey band is the $68 \%$ confidence region of a theoretical Harrison-Zeldovich $n=1$ correlation function of amplitude $Q_{r m s-P S}=15.4 \mu \mathrm{K}$. This is from a $\chi^{2}$ fit using a Gaussian approximation to the DMR beam. For a more detailed discussion of $n$ and $Q_{r m s-P S}$ fits see Chapter 10.

Smoot et al. (1992) and Wright et al. (1992) discuss the rms temperature fluctuations on a $10^{\circ}$ scale, $\sigma_{s k y}\left(10^{\circ}\right)$. As pointed out in Section $4.7, C_{A x B}(0)=\sigma^{2}\left(7^{\circ}\right)_{s k y}$ when identical weighting and multipole subtraction techniques are used. 


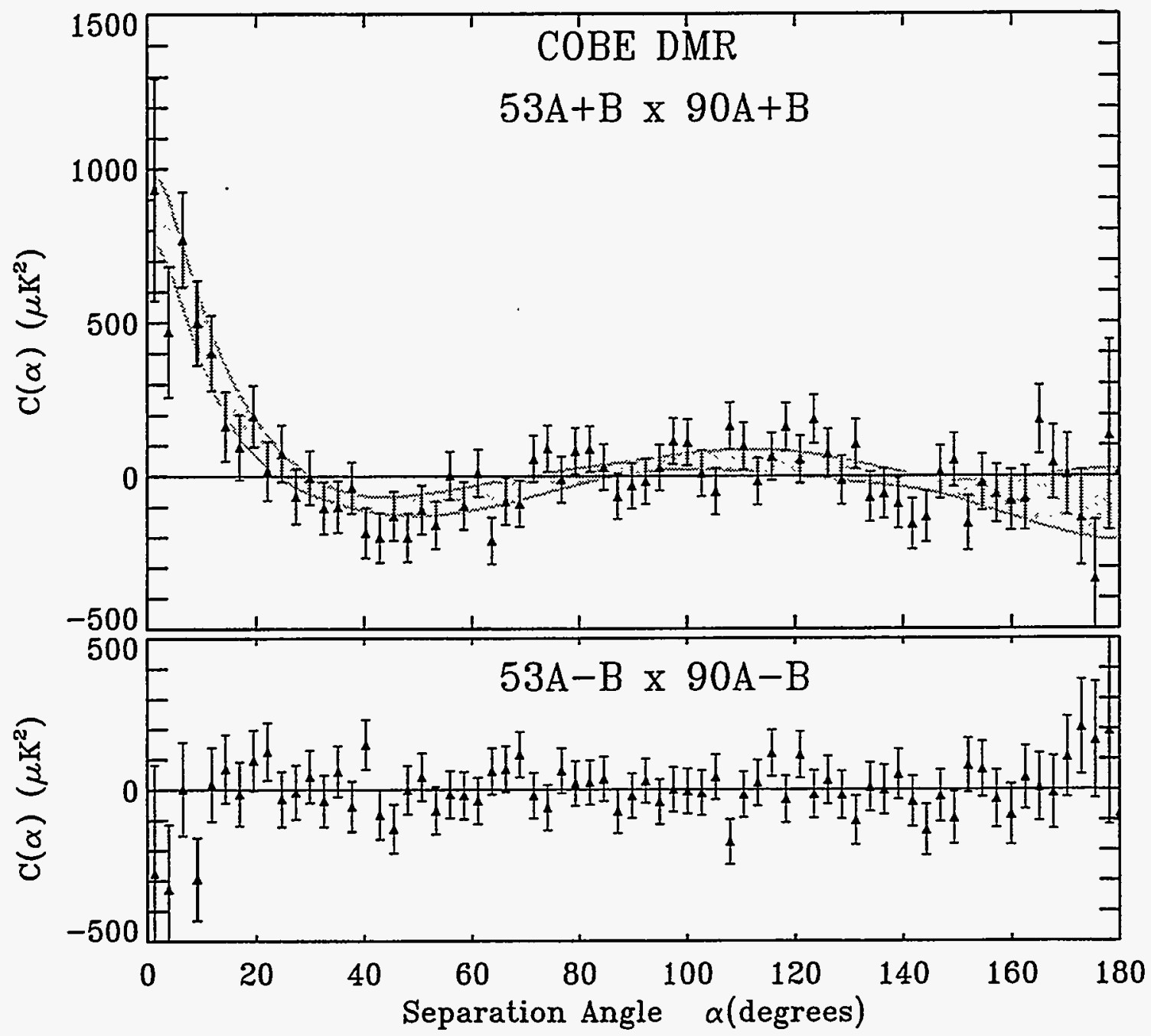

Figure 5.5: Yr1 Cross-correlation Function of $53 \mathrm{GHz}$ with $90 \mathrm{GHz}$ for $|b|>20^{\circ}$, plus the $68 \%$ confidence level cosmic variations from a scale-invariant-spectrum correlation function with an expected $Q_{\tau m s-P S}=15.4 \mu \mathrm{K}$. Top is for the sum maps, and bottom is for the difference maps (Smoot et al. 1992, Figure 3). 
The first year unweighted $53 \mathrm{~A} \times \mathrm{B}$ correlation functions from galcuts of $20^{\circ}, 30^{\circ}$ and $40^{\circ}$ (weighted average) yields an rms temperature fluctuation of $\sqrt{C_{m d}(0)}=46 \pm 6 \mu \mathrm{K}$, where "md" means that the best fit mean and dipole were removed before $C(\alpha)$ was accumulated and the error bars are noise-only. The weighted correlation function yields $39 \pm 8 \mu \mathrm{K}$. See Table 7.3 for a more complete comparison of rms values derived from $\sqrt{C(0)}$.

\subsection{First Year Dipole Results}

The two largest features in the DMR skymaps are the dipole and the Galaxy. The Galaxy is complicated and difficult to remove while the dipole is simple, at least in principle. Observers with velocity $\vec{\beta}=\vec{v} / c$ through a Planckian radiation field of temperature $T_{o}$ will measure directionally dependent temperatures,

$$
T_{o b s}(\theta)=T_{o} \frac{\left(1-\beta^{2}\right)^{1 / 2}}{(1-\beta \mu)}
$$

where $\mu=\cos \theta$ and $\theta$ is the angle between $\vec{\beta}$ and the direction of observation as measured in the observer's frame (Peebles \& Wilkinson 1968). We expand this through order $\beta^{3}$ to show that the dipole is the largest member of a family of kinematic anisotropies,

$$
\frac{\Delta T}{T_{o}}=\beta \mu+\frac{\beta^{2}}{2}\left(2 \mu^{2}-1\right)+\frac{\beta^{3}}{4}\left(4 \mu^{3}-2 \mu\right)+O\left(\beta^{4}\right) .
$$

The six DMR channels are consistent with a Doppler-shifted isotropic blackbody radiation field with a dipole amplitude $\Delta T=3.365 \pm 0.027 \mathrm{mK}$ toward direction $\left(l^{I I}, b^{I I}\right)=$ $264.4^{\circ} \pm 0.3^{\circ}, 48.4^{\circ} \pm 0.5^{\circ}$ (Kogut, Lineweaver et al. 1993). The errors on the amplitude are dominated by the absolute calibration uncertainty while the errors on the direction are dominated by the uncertainty of the Galactic model. Next in importance are the magnetic susceptibility corrections and the noise. Compared to the previous DMR value (Smoot et al. 1990) the amplitude increased by $5 \mu \mathrm{K}$ and the error bars on the amplitude decreased by a factor of 3 . The direction changed by half a degree and the errors in the direction improved by half a degree. Better modeling of the Galaxy has led to this improvement.

Interpreting this dipole as purely kinematic and using $T_{o}=2.73 \mathrm{~K}$, the derived velocity of the Sun with respect to the CMB is $369.5 \pm 3.0 \mathrm{~km} \mathrm{~s}^{-1}$. In Table 5.1 we decompose the velocity of the observer (i.e., the COBE satellite) with respect to the CMB into a sum of nested velocities associated with structures of increasing size (see Kogut, Lineweaver et al. 1993, Table 3). The velocities quoted have been used to derive the Local Group velocity 
with respect to the CMB: $\vec{v}_{L G}=627 \pm 22 \mathrm{~km} \mathrm{~s}^{-1}$ toward $\left(l^{I I}, b^{I I}\right)=276^{\circ} \pm 3^{\circ}, 30^{\circ} \pm 3^{\circ}$. Figure 5.6 is a map of the directions and velocities in Table 5.1.

Equation (5.2) predicts a dipole pattern with no frequency dependence. If the observed dipole were larger at $31 \mathrm{GHz}$ than at $90 \mathrm{GHz}$ one could eliminate the kinematic interpretation in favor of Galactic synchrotron and/or inhomogeneity in the inter-stellar medium or inter-galactic medium. Modelling the frequency dependence of the DMR dipole amplitudes as $\nu^{p}$ we obtain $p=0.004 \pm 0.017$. Thus the frequency independence of both the FIRAS (Fixsen et al.1994) and DMR results represents a successfully passed consistency test of the Doppler-shifted Planckian assumption of equation (5.2).

During the past decade studies of bulk motion (Bertschinger et al.1990) as well as cumulative acceleration analyses in the IR, optical and X-ray have produced consistent and independent support for the standard kinematic interpretation of the CMB dipole (e.g. Strauss et al.1992). The broadstroke picture is simple. Virgo-centric infall with a bulk velocity towards the "Great Attractor" (Dressler et al.1986) can account qualitatively and within certain limits quantitatively for both the direction and the magnitude of the velocity of the Local Group as inferred from the CMB dipole.

The inferred velocity of our Local Group is consistent with the peculiar velocity distribution of other groups. This velocity distribution is the result of the influence of potential fluctuations like those which have been measured on the surface of last scattering by the DMR (Smoot et al. 1992). These fluctuations $\left(Q_{r m s}-P S \approx 17 \mu \mathrm{K}, n \approx 1\right)$ are at a level consistent with the production of the velocity distributions observed (see Górski 1991, Scaramella 1992, and Kogut, Lineweaver et al. 1993).

\subsubsection{Calibrator of Other Diffuse Backgrounds}

The standard model predicts that the rest frame of the distant sources of other diffuse backgrounds will coincide with the rest frame of the CMB. If the CMB dipole is kinematic then it can serve as a calibrator for the expected dipoles in these other backgrounds. The generalization of equation (5.2) for motion through an isotropic but not necessarily Planckian radiation field of intensity $I_{o}(\nu)$ is the observed intensity anisotropy

$$
\frac{\Delta I}{I_{o}}(\nu, \theta)=\frac{I_{o b s}(\nu, \theta)-I_{o}(\nu)}{I_{o}(\nu)},
$$

where $\nu$ is the frequency in the observer's frame and $I_{0}$ is the intensity in the rest frame of the radiation. The result to third order in $\beta$ is 


$$
\begin{aligned}
\frac{\Delta I}{I_{o}}= & \beta \mu\left(3-\alpha_{1}\right)+\frac{\beta^{2}}{2}\left[2 \mu^{2}\left(6-3 \alpha_{1}+\frac{\alpha_{2}}{2}\right)-\left(3-\alpha_{1}\right)\right] \\
& +\frac{\beta^{3}}{4}\left[4 \mu^{3}\left(10-6 \alpha_{1}+\frac{3}{2} \alpha_{2}-\frac{1}{6} \alpha_{3}\right)-2 \mu\left(9-5 \alpha_{1}+\alpha_{2}\right)\right]+O\left(\beta^{4}\right)
\end{aligned}
$$

where $\alpha_{n}=\frac{\nu^{n}}{I(\nu)} \frac{\partial^{n} I(\nu)}{\partial \nu^{n}}$. A pedagogical check of this formula can be made by noticing that for a Planckian spectrum $\Delta I / I_{o}=\Delta T_{a n t} / T_{a n t}=\frac{x e^{x}}{e^{x}-1} \Delta T / T_{o}$, where $T_{a n t}$ is antenna temperature and $x=h \nu / k T_{\circ}$ (Appendix G). In the Rayleigh-Jeans limit, $\alpha_{1}=\alpha_{2}=2$, $\alpha_{3}=0$ and one obtains $\Delta I / I_{0}=\Delta T / T_{0}$. An analogous simplification does not occur in the Wien limit because of the $\nu$ dependence of the derivatives $\alpha_{n}$. Another check is that an $I \propto \nu^{3}$ non-Planckian spectrum yields no kinematic anisotropy $\left(\Delta I / I_{o}=0\right)$ since $I / \nu^{3}$ is a Lorentz invariant. For this case, $\alpha_{1}=3$ and $\alpha_{2}=\alpha_{3}=6$.

A measurement of the dipole anisotropy from the DMR first-year skymaps implies a Local Group velocity with respect to the CMB rest frame of $\overrightarrow{v_{G} G}=627 \pm 22 \mathrm{~km} \mathrm{~s}$ toward $\left(l^{I I}, b^{I I}\right)=\left(276^{\circ} \pm 3^{\circ}, 30^{\circ} \pm 3^{\circ}\right)$. The relevant data is consistent enough to define a testable "standard kinematic interpretation" of this dipole (Lineweaver et al. 1994a). A confirmed detection of an X-ray background dipole at the level determined by equation (5.4) in agreement with the CMB dipole would provide a substantial confirmation of the standard interpretation. 

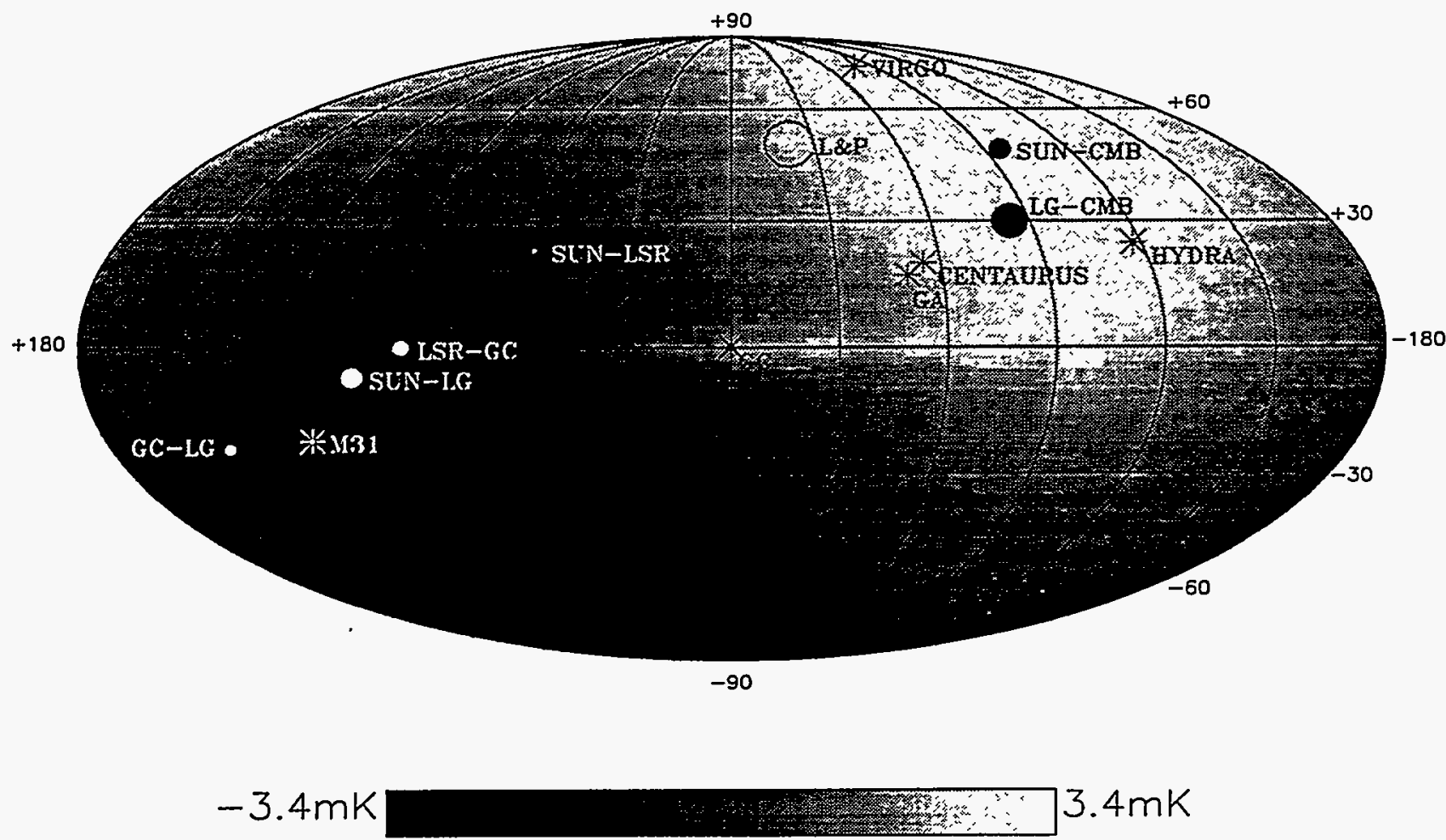

Figure 5.6: Full-sky map in Galactic coordinates showing the velocities from Table 5.1. The size of the dot is roughly proportional to the magnitude of the the velocity. The motion of the Sun around the Galactic center as well as the motion of the Galaxy with respect to the Local Group are $\sim 180^{\circ}$ away from the $\sim 370 \mathrm{~km} \mathrm{~s}^{-1}$ SUN-CMB dipole apex. The circle labeled " $\mathrm{L} \& \mathrm{P}$ " is the Lauer \& Postman (1992) result. "GA" is the Great Attractor.

Table 5.1: Nested Velocities

\begin{tabular}{ccccc}
\hline Object & Frame & $\begin{array}{c}\text { Velocity } \\
\left(\mathrm{km} \mathrm{s}^{-1}\right)\end{array}$ & $\begin{array}{c}\text { Direction }\left(l^{I I}, b^{I I}\right) \\
\text { (degrees) }\end{array}$ & Reference \\
\hline COBE & Earth & $7.4 \pm 0.1$ & $\sim \perp$ ecliptic plane & \\
Earth & Sun & $29.8 \pm 0.5$ & ecliptic plane & Astronomical Almanac, 1990 \\
Sun & LSR & $20.0 \pm 1.4$ & $57 \pm 4,23 \pm 4$ & Kerr \& Lynden-Bell, 1986 \\
LSR & GC & $222.0 \pm 5.0$ & $91.1 \pm 0.4,0$ & Fich, Blitz \& Stark, 1989 \\
GC & LG & $112.3 \pm 24$ & $145.7 \pm 6,-23.8 \pm 5$ & combine rows $3,4,8$ \\
LG & CMB & $627 \pm 22$ & $276 \pm 3,30 \pm 3$ & combine rows 7,8 \\
Sun & CMB & $369.5 \pm 3.0$ & $264.4 \pm 0.3,48.4 \pm 0.5$ & Kogut et al. 1993 \\
Sun & LG & $308 \pm 23$ & $105 \pm 5,-7 \pm 4$ & Yahil et al. 1977 \\
\hline
\end{tabular}

(LSR: Local Standard of Rest, GC: Galactic Center, LG: Local Group) 


\title{
Chapter 6
}

\section{Correlation Function Error Analysis}

\begin{abstract}
We compute error bars for $C(\alpha)$ from a standard propagation of errors formula and compare the results with a calculation of the covariance matrix of the correlation function with and without cosmic variance. Approximations for the error bar dependence on separation angle, Galactic plane cut and time are given.
\end{abstract}

\subsection{Introduction}

Since 1992 when Smoot et al.(1992) and Wright et al.(1992) presented preliminary correlation function results from the COBE DMR first year skymaps, the correlation function has become an important standard tool for testing models (Adams et al.1992, Scaramella \& Vittorio 1992, Seljak \& Bertschinger 1993, Bennett et al. 1994) and comparing data (Ganga et al. 1993). The errors on $C(\alpha)$ can be important factors in determining the power spectrum parameters $n$ and $Q_{r m s-P S}$. We are thus motivated to present a closer look at the errors on the correlation functions of the DMR maps.

First we need to specify what exactly we want error bars for. The measured $C(\alpha)$ can be construed as an estimate of the correlation function of either (i) our horizon volume, or (ii) the ensemble average of all horizon volumes $\langle C(\alpha)\rangle$. For (i) the error bars on $C(\alpha)$ are from the uncertainty in our estimation of the true temperatures $T_{i}$ of our observed 
sky. These are the temperatures which would be measured in a perfect noiseless instrument and cosmic variance plays no role. For (ii) the error bars on $C(\alpha)$ need to include the additional uncertainty due to cosmic variance. Case (i) is useful when comparing data sets while case (ii) is useful when comparing data with models but depends on the ensemble average $\langle C(\alpha)\rangle$ of the model one is trying to specify.

When we apply error bars without including cosmic variance to $C(\alpha)$, we are implicitly interpreting $C(\alpha)$ as an estimate of our sky's $C(\alpha)$, not $\langle C(\alpha)\rangle$. When we include cosmic variance we have to assume the presence of structure at a chosen level. When formulae for the errors are derived from covariance matrices without cosmic variance, the results are dependent on the unknown true temperatures of the sky $T_{i}$. With cosmic variance, the results are dependent on the unknown ensemble average $\langle C(\alpha)\rangle$. The standard propagation of error formula discussed below has the advantage of depending only on the actual temperatures in the observed map and, for reasonable values of the parameters $n$ and $Q_{r m s-P S}$, yields a result in between noise-only error bars and error bars including cosmic variance (see Figure 6.1).

\subsection{Error Bars and Covariance Matrices of $C(\alpha)$}

Unless specifically described as noise-only, the errors on the correlation functions in this thesis were derived from the standard propagation of errors formula,

$$
\sigma_{c}^{2}(\alpha)=\sum_{m}\left(\frac{\partial C(\alpha)}{\partial T_{o b s, m}} \sigma_{m}\right)^{2}
$$

where the sum is over all pixels in both maps and $\sigma_{m}^{2}=1 / w_{m}=\sigma_{c h}^{2} / N_{m}$ is an estimate of the pixel noise. $N_{m}$ is the number of times pixel $m$ has been observed. This leads to (see Appendix K)

$$
\sigma_{c}^{2}(\alpha)=\sum_{w}\left[w_{i} T_{o b s, i}^{2}+w_{j} T_{o b s, j}^{2}\right]
$$

where the operator $\sum_{w} \equiv \frac{1}{W^{2}} \sum_{i, j} \delta\left(\alpha-\alpha_{i j}\right) w_{i} w_{j}$ and $W \equiv \sum_{i, j} \delta\left(\alpha-\alpha_{i j}\right) w_{i} w_{j}$. The delta function forces the sum to run over all pixel pairs $i j$ whose separation angle $\alpha_{i j}$ lies within half a bin width of $\alpha$. It is important to notice that this is a function only of the observed temperatures $T_{o b s, i}$ and we make no assumptions about an underlying CMB temperature field $T_{i}$.

Equation (6.2) can be compared to the variances contained in the diagonal elements of 
the covariance matrix

$$
\sigma^{2}(\alpha, \beta)=<\Delta C(\alpha) \Delta C(\beta)>
$$

where $\alpha$ and $\beta$ are separation angles, $\Delta C(\alpha)=C_{r}(\alpha)-\left\langle C_{r}(\alpha)\right\rangle$ and the angle brackets are averages over the ensemble of realizations indexed by $r$. The $T_{i}$-dependent diagonal of the covariance matrix is $\sigma^{2}(\alpha) \equiv \delta_{\alpha \beta} \sigma^{2}(\alpha, \beta)$. For the case where cosmic variance is not included, after lengthy algebra, we obtain

$$
\sigma_{c}^{2}(\alpha)=\sum_{w}\left[1+w_{i} T_{i}^{2}+w_{j} T_{j}^{2}+\sum_{k, k \neq i} \delta\left(\alpha-\alpha_{j k}\right) w_{k} T_{i} T_{k}+\sum_{l, l \neq j} \delta\left(\alpha-\alpha_{i l}\right) w_{l} T_{j} T_{l}\right],
$$

where $i$ and $k$ are indices for map 1 and $j$ and $l$ are indices for map 2. Equation (6.4) is a function of the true but unknown temperatures $T_{i}$. The " 1 " is the noise-noise term while the others are noise-signal terms. For each angle $\alpha$, the angles $\alpha_{i k}$ and $\alpha_{j l}$ take on all values in the range $(0,2 \alpha]$. Therefore the last two terms pick up positive contributions from that part of the sum where $\alpha_{i k}$ and $\alpha_{j l}$ are $\lesssim 15^{\circ}$ (using the approximation that $C(\alpha)$ averaged over the range $15^{\circ}<\alpha<2 \alpha$ equals zero). These last two terms are responsible for the bumps and wiggles in the no-cosmic-variance results of Figure 6.1.

To compare equation (6.2) with (6.4) we write the map temperatures as a sum of the true temperatures plus noise: $T_{o b s, i}=T_{i}+n_{i}$ where $n_{i}$ is from a normal distribution of 0 mean and variance $\sigma_{i}^{2}$

$$
\begin{aligned}
& n_{i} \in N\left(0, \sigma_{i}^{2}\right), \sigma_{i}^{2}=\sigma_{c h}^{2} / N_{i} \\
& T_{i} \in N\left(0, \sigma_{s k y}^{2}\right) .
\end{aligned}
$$

Thus equation (6.2) becomes

$$
\sigma_{c}^{2}(\alpha)=\sum_{w}\left[2+w_{i} T_{i}^{2}+w_{j} T_{j}^{2}\right]
$$

where we have used the fact that averaged over $i, n_{i} T_{i} \approx 0$ and $n_{i}^{2} \approx \sigma_{i}^{2}$. With these approximations equation (6.7) has a "noise-noise" term twice as large as the corresponding term in the no-cosmic variance covariance expression of equation (6.4).

As a pedagogical check we can also compare equations (6.4) and (6.7) with the diagonal of the covariance matrix when cosmic variance is included

$$
\sigma_{c}^{2}(\alpha)=\sum_{w}\left[1+w_{i} C(0)+w_{j} C(0)+\sum_{k, k \neq i} w_{k} C_{i k}+\sum_{l, l \neq j} w_{l} C_{j l}+\sum_{k l} \delta\left(\alpha-\alpha_{k l}\right) w_{k} w_{l} C_{i k} C_{j l}\right],
$$


where the $C(0)$ terms are unweighted correlation functions at zero lag. The first term in the square bracket is the noise-noise term. The next four are the noise-signal terms and the last is the signal-signal term unique to simulations with cosmic variance. Notice that the $w C(0)$ terms correspond to the $w T^{2}$ in equations (6.4) and (6.7) and the $w C_{i k}$ terms correspond to the $w T T$ terms in equation (6.4). Notice that neither the map temperatures $\left(T_{o b s, i}\right)$ nor the true sky temperatures $\left(\mathcal{T}_{i}\right)$ appear since the ensemble average correlation function for a given angular separation $C_{i j} \equiv<C\left(\alpha_{i j}\right)>$ is specified by the model but the temperatures are not. Thus, both the "noise-signal" and "signal-signal" error terms are a function of $C_{i j}$.

In this thesis, the displayed correlation function errors are from

$$
\sigma_{c}^{2}(\alpha)=\frac{\sum_{i j} \delta\left(\alpha-\alpha_{i j}\right)\left[\sigma_{c h 1}^{2} N_{j} N_{i}^{2} T_{o b s, i}^{2}+\sigma_{c h 2}^{2} N_{i} N_{j}^{2} T_{o b s, j}^{2}\right]}{\left(\sum_{i j} \delta\left(\alpha-\alpha_{i j}\right) N_{i} N_{j}\right)^{2}}
$$

which is derived from the standard error propagation formula in Appendix K. In Figure 6.1 the curve labeled 'conservative' comes from equation (6.9). It is symmetric about $\alpha=90^{\circ}$ and shows no bumps or wiggles. When the error bars are described as noise-only they are computed from (Appendix $\mathrm{K}$ )

$$
\sigma_{c}^{2}(\alpha)=\frac{\sigma_{c h 1}^{2} \sigma_{c h 2}^{2}}{\sum_{i j} N_{i} N_{j}}
$$

The noise-only error bars of equation (6.10) are also displayed in Figure 6.1. Simulations of $n=1$ and $Q_{r m s-P S}=17 \mu \mathrm{K}$ without cosmic variance (equation (6.4)) and with cosmic variance (equation (6.8)) yield respectively the dashed and dot-dashed curves in Figure 6.1. For auto-correlations the results are identical to those presented above except the variance is twice as large $\sigma_{c}^{2} \rightarrow 2 \sigma_{c}^{2}$.

In summary, the error bars displayed on $C(\alpha)$ depend on what exactly one wants to represent. Analogous "noise-noise" and "noise-signal" terms are present in the three methods discussed. Error bars derived from covariance matrices of Monte Carlo simulations are necessarily model dependent. The standard propagation of error formula has the advantage of depending only on the actual temperatures in the observed map and, for reasonable values of the parameters $n$ and $Q_{r m s-P S}$, yields a result in between noise-only error bars and error bars including cosmic variance (see Figure 6.1). 


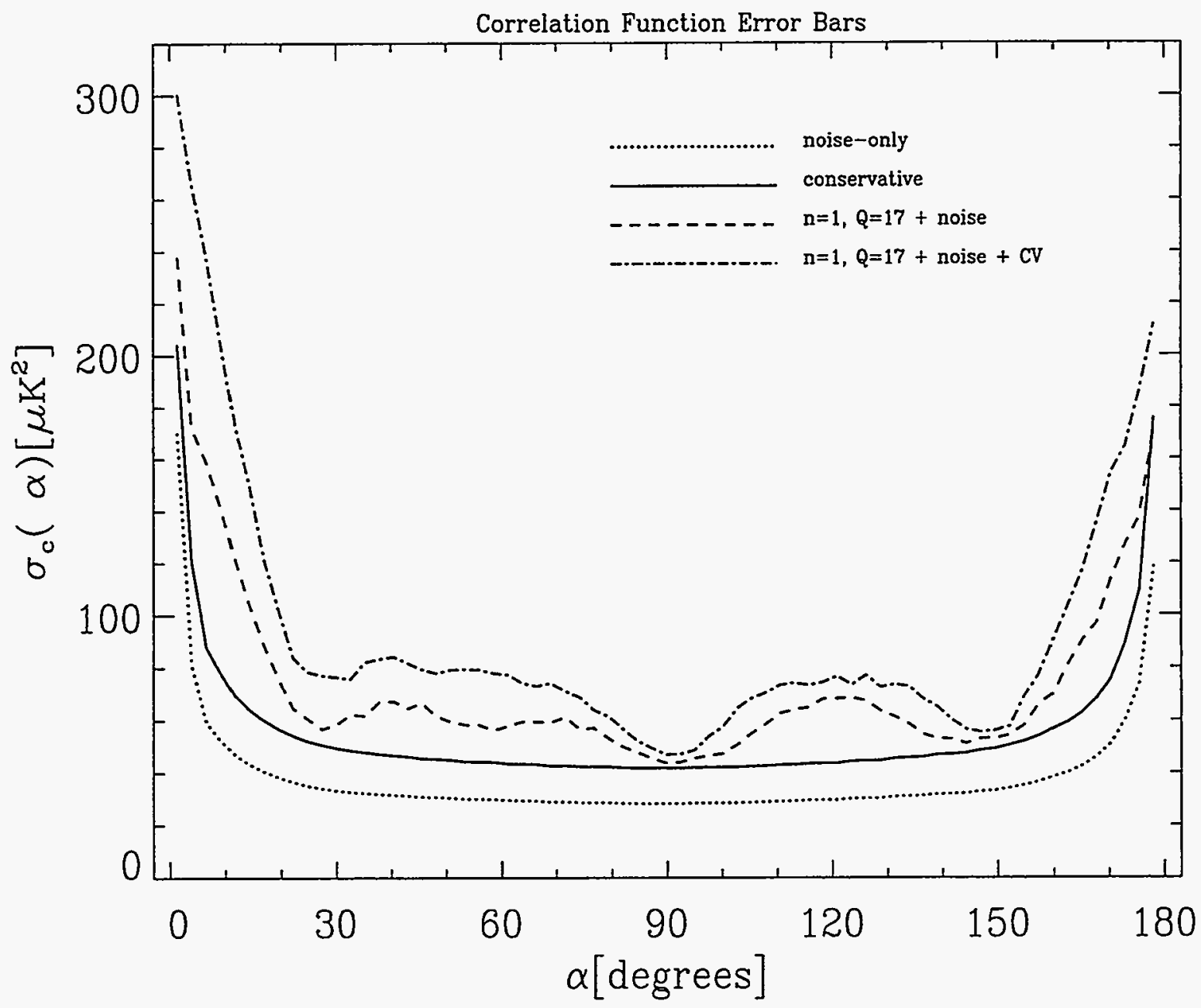

Figure 6.1: Error bars on the 2 Yr cross-correlation $53 \mathrm{GHz}$ x $90 \mathrm{GHz}$ from the standard propagation of errors formula (labeled 'conservative' and computed from equation (6.9)), compared with noise-only error bars (equation (6.10)). Also shown are error bars derived from $68 \%$ confidence levels on $C(\alpha)$ from 2000 simulations of $n=1, Q_{r m s-P S}=17 \mu \mathrm{K}$ structure + noise, with and without cosmic variance (CV). A $20^{\circ}$ Galactic plane cut has been made and the best fit mean, dipole and quadrupole have been subtracted. 


\subsection{Separation Angle, Galactic Cut and Time Dependence}

The most obvious feature of $\sigma_{c}(\alpha)$ is that it has a maximum at small and large values of $\alpha$ and a minimum at intermediate $\alpha$ values. This is due to the relative number of pixel pairs as a function of separation angle $\alpha$. For the dominant noise-noise term, the $\alpha$ dependence is,

$$
\sigma_{c}(\alpha) \propto \sum_{w} \alpha \frac{1}{\sqrt{\sin (\alpha)}}
$$

The errors on $C(\alpha)$ also depend on the sky coverage. Typically, Galactic latitude cuts of $|b|<10^{\circ}, 20^{\circ}$ and $30^{\circ}$ are used to remove Galactic contamination. The Galactic cut determines the number of pairs $i, j$ to sum over. Therefore

$$
\sigma_{c}^{2}(\alpha,|b|) \propto \sum_{w} \propto \frac{1}{\Omega}
$$

where $\Omega$ is the solid angle of sky coverage. Since $\Omega \propto(1-\sin (b))$ we have,

$$
\sigma_{c}(\alpha) \propto \frac{1}{\sqrt{1-\sin (b)}}
$$

Monte Carlo simulations at various Galactic cuts have verified this analytic result. For example a $30^{\circ}$ Galactic cut increases $\sigma_{c}^{2}(\alpha)$ by a factor of two over the no Galactic cut values.

One can make a crude estimate of the time dependence of the error bars. Consider the time dependence of each of the terms in equation (6.8). The number of observations of

a pixel $N_{i}$ increases linearly with observation time $N_{i} \propto t$. Since $w_{i} \equiv \frac{1}{\sigma_{i}^{2}}=\frac{N_{i}}{\sigma_{c h}^{2}} \propto t$, we have, $\sum_{w} \propto 1 / t^{2}$. The " 1 " is the noise-noise term. The correlations $C(0)$ and $C_{i k}$ are timeindependent constants: $C(0) \approx 1000 \mu \mathrm{K}$ and and the $w C_{i k}$ sums are about three times the $w C(0)$ terms. Assuming uncorrelated noise and 2 years of observation $\sigma_{i}=\frac{\sigma_{c h}}{\sqrt{N_{i}}} \approx 100 \mu \mathrm{K}$. Therefore the sum of the noise-signal terms is $\frac{8 C(0)}{\sigma_{i}^{2}} \sim 1$. The rough $t$ dependence of $\sigma_{c}^{2}(\alpha)$ from equation (6.8) is then

$$
\sigma_{c}^{2}(\alpha) \propto \frac{1}{t^{2}}\left[1+\left(\frac{t}{t_{2 y r}}\right)+x\left(\frac{t}{t_{2 y r}}\right)^{2}\right],
$$

where $x \sim 1$ and has an $\alpha$ dependence which modulates the signal-signal cosmic variance error bars. This modulation can be seen as the variation of the width of the grey band in Figure 7.6.

For small $t$, the noise-noise terms dominates and $\sigma_{c}(\alpha) \propto 1 / t$. When the noisesignal term dominates, $\sigma_{c}(\alpha) \propto 1 / \sqrt{t}$ and when the signal-signal term dominates, $\sigma_{c}(\alpha) \approx$ 
constant. After two years of operation, the error bars on $C(\alpha)$ from the best combination maps are no longer dominated by the $1 / t$ behavior of the noise regime but are decreasing more slowly with time. The size of the error bars in the final two years decreases even more slowly.

This time-dependence approximation is very crude because we have glossed over the $\alpha$ dependence of the noise-signal and signal-signal components. For example look at the errors in Figure 6.1. At $\alpha \approx 90^{\circ}$ and $150^{\circ}$ the effect of cosmic variance is very small compared to the noise while at $\alpha \approx 10^{\circ}$ cosmic variance is very large. 


\title{
Chapter 7
}

\section{Correlation Function Analysis of the Two Year Maps}

\begin{abstract}
We present the results of a detailed correlation function analysis of the two year (2Yr) maps. The six separate DMR channels are mutually consistent. The $A+B$ and $A-B$ comparison for each frequency indicates a CMB signal that is approximately frequency independent. The signal is very robust to Galactic latitude cuts of $20^{\circ}, 30^{\circ}$ and $40^{\circ}$. These results support the conclusions based on the first year of data. The statistical significance of the detection in the two year correlation function is $>18 \sigma$. The rms temperature fluctuations obtained from $\sqrt{C(0)}$ for both weighted and unweighted cases as a function of Galactic latitude cut are tabulated in Table 7.3. Although the technique used to obtain them differed, our results are consistent with the Bennett et al. (1994) results. We find for the two year data set crosscorrelation of $53 \mathrm{GHz}$ with $90 \mathrm{GHz}$ rms temperature fluctuations of $\sqrt{C(0)}=35 \pm 4 \mu \mathrm{K}$. The results of a novel method to obtain the rms quadrupole of our sky are given. The $Q_{\text {rms }}$ obtained from the two year $53 \times 90$ correlation function is $9 \pm 8 \mu \mathrm{K}$.
\end{abstract}

\subsection{Channel Comparison, $A+B$ and A-B Comparison}

It is important that the reported CMB signal be seen consistently in each channel and consistently from year to year. The signal should show no frequency dependence or Galactic latitude cut dependence. These properties can be conveniently checked and quantified using 


\section{Separate Channels $2 Y r$}

Channel A
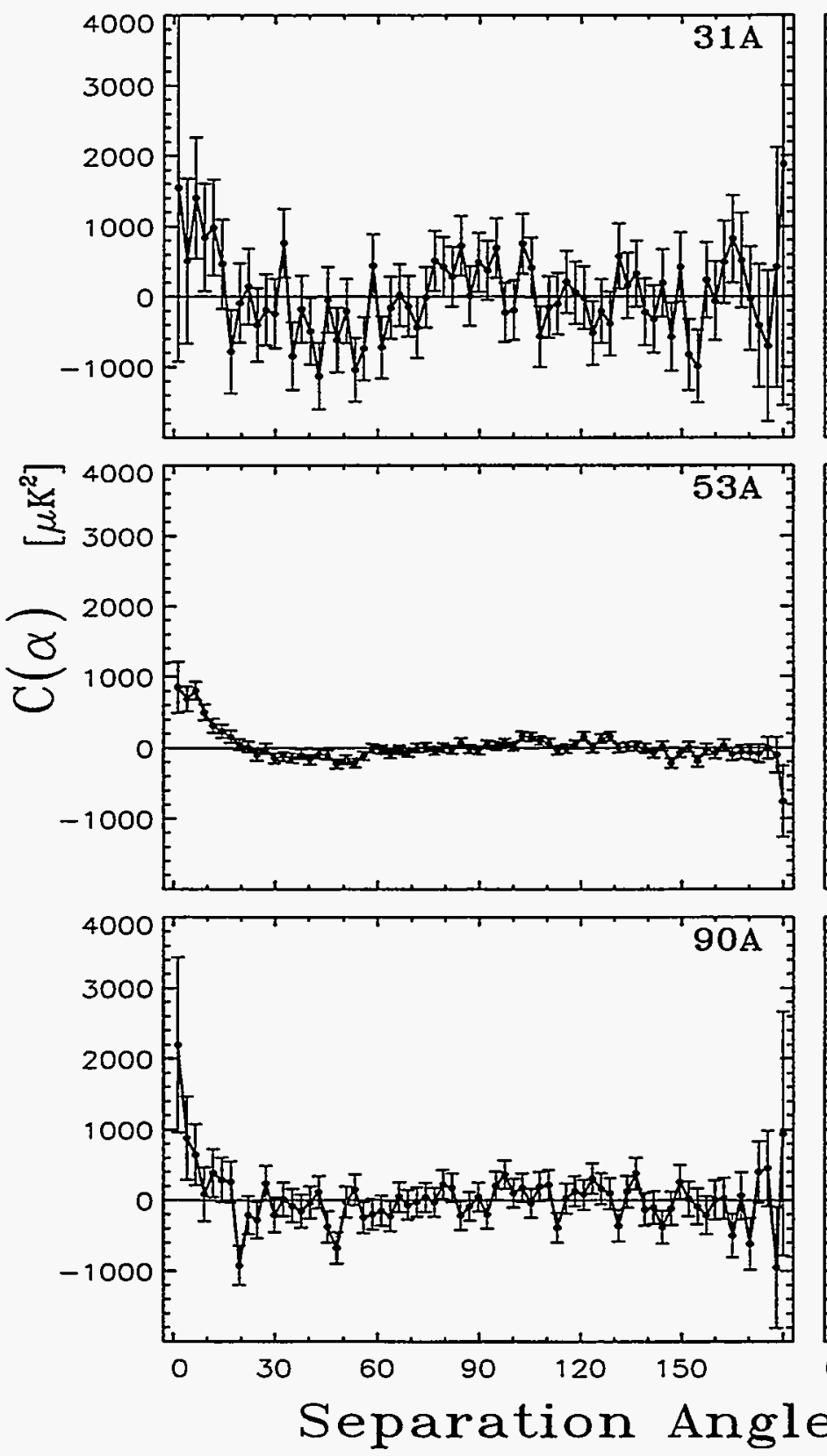

Channel $\mathrm{B}$
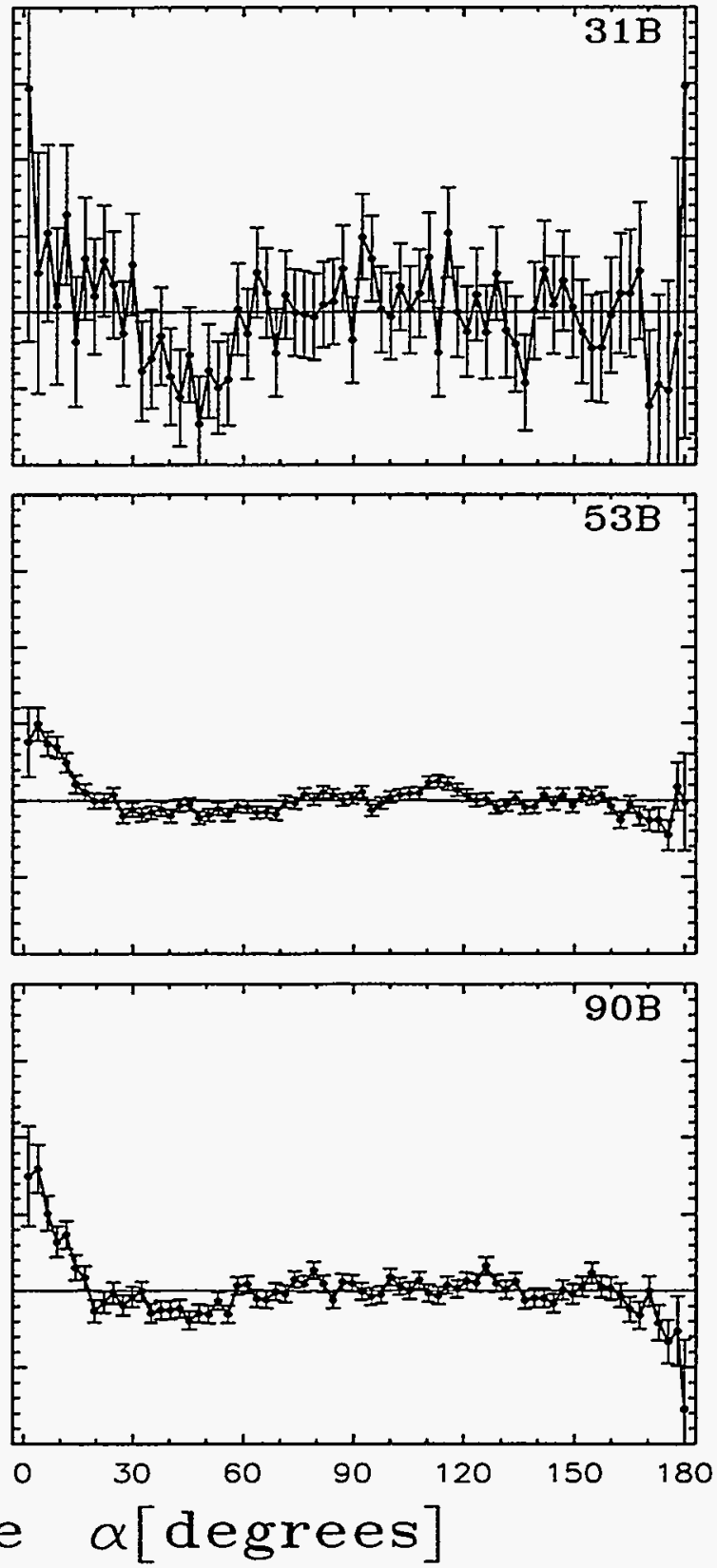

Figure 7.1: 2Yr Separate Channel Comparison. Each channel is consistent with a small angular scale positive correlation. These are auto-correlations in the region $|b|>20^{\circ}$ and have had the best fit mean, dipole and quadrupole removed. 


\section{Auto-Correlations $2 Y r$}

$\mathrm{A}+\mathrm{B}$
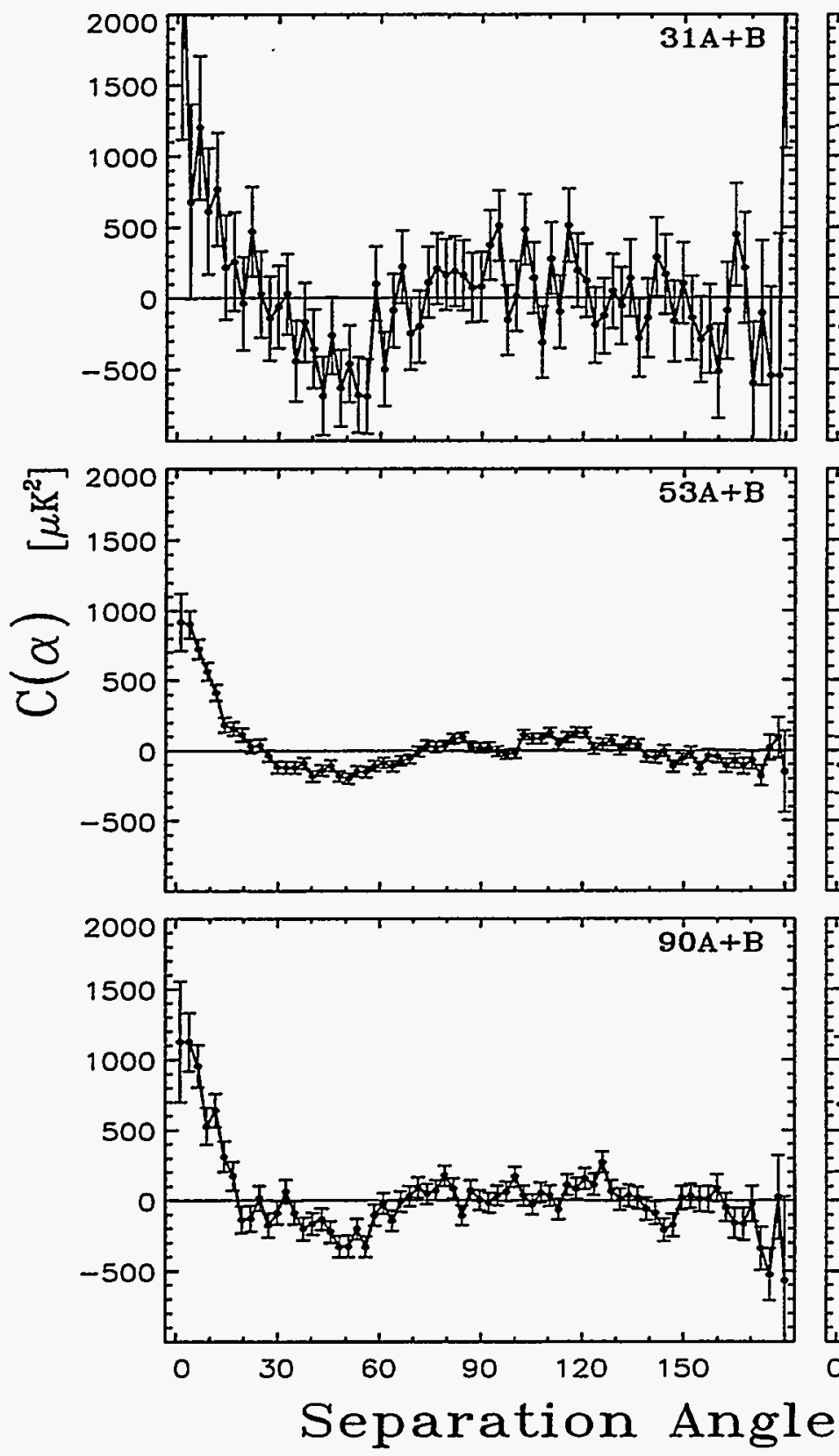

$\mathrm{A}-\mathrm{B}$
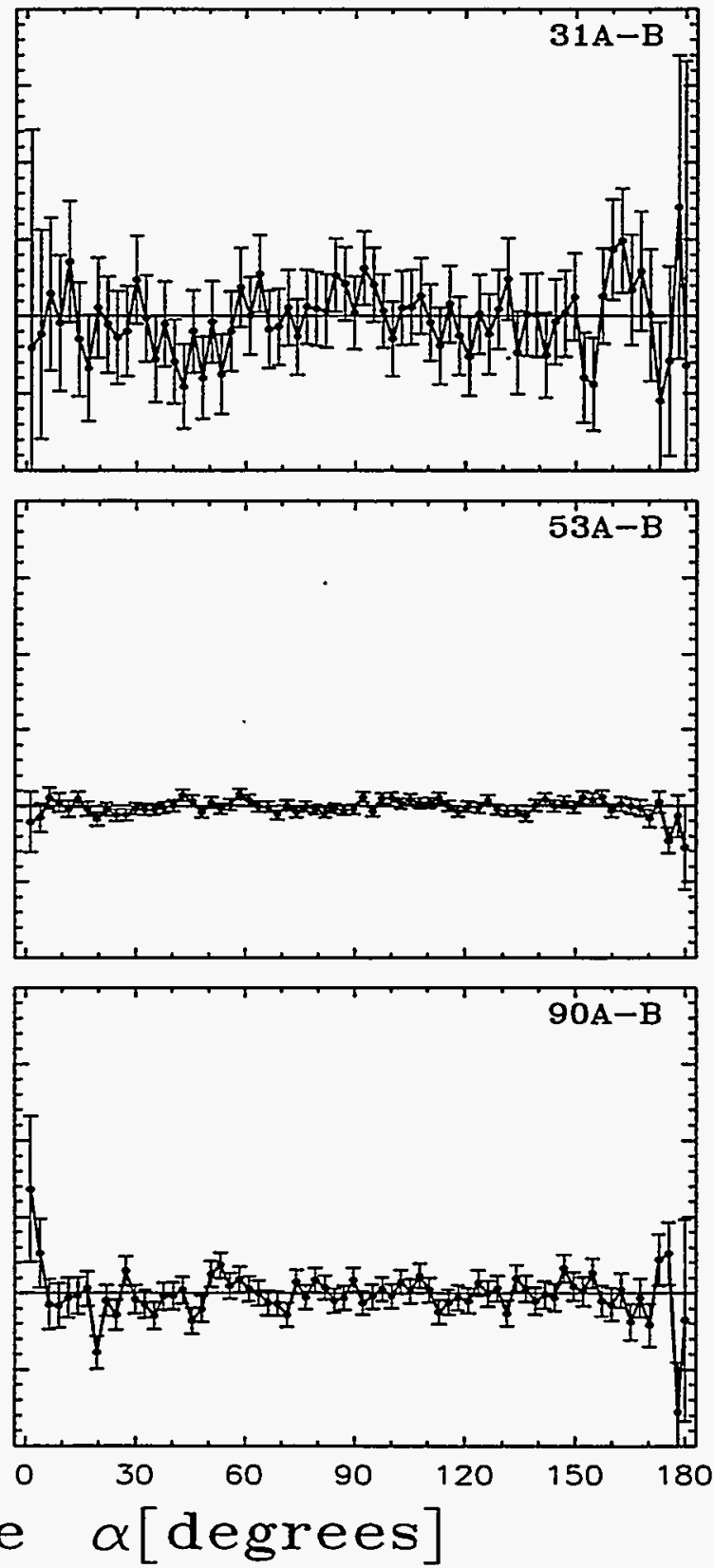

Figure 7.2: $2 Y \mathrm{Y} A+B$ vs $A-B$ Comparison. As expected of a true CMB signal, the $A+B$ versions display a signal at approximately the same level for each frequency while the A-B versions are consistent with no signal. These are auto-correlations in the region $|b|>20^{\circ}$ and have had the best fit mean, dipole and quadrupole removed. 
correlation functions. In the following correlation function analysis of the $2 \mathrm{Yr}$ data we will use only maps from the pass 2 software processing (Appendix E).

Figure 7.1 is a comparison of the $2 \mathrm{Yr}_{\mathrm{r}}$ separate channel auto-correlation functions. The traces in each channel are consistent with a slowly rising positive correlation as $\alpha$ decreases, peaking at a $C(0) \sim 1000 \mu \mathrm{K}^{2}$.

For each frequency, Figure 7.2 compares the auto-correlations of $A+B$ and $A-B$ maps. As expected for true CMB structure, the $C(0) \sim 1000 \mu \mathrm{K}^{2}$ signal is present in each of the $A+B$ plots at approximately the same level and is absent in the A-B plots. The fact that the level is approximately frequency independent is important. If we were observing Galactic synchrotron or free-free emission the $31 \mathrm{GHz}$ signal would be $>9$ times higher than the $53 \mathrm{GHz}$ signal. Figure 7.2 shows that this is clearly not the case.

In antenna temperature we have $T_{i}(\nu) \propto \nu^{\beta_{i}}$ where the three main components of Galactic foreground (subscript $i$ ) are synchrotron, free-free and dust with $\beta_{\text {sync }} \approx$ $-2.8, \beta_{f f} \approx-2.1$ and $\beta_{d u s t} \approx 1.5$ (see Section 2.3). Since $C(\alpha) \propto T^{2}$, we can obtain the frequency dependence of the signal by fitting each bin of the correlation function to the model

$$
C(\alpha, \nu)=A \nu^{2 \beta}
$$

using the values from the $\mathrm{A}+\mathrm{B} 31 \mathrm{GHz}, 53 \mathrm{GHz}$ and $90 \mathrm{GHz}$ correlation functions. The signal in the small $\alpha$ region where the correlations functions are positive yields $\beta=-0.1 \pm 0.5$ (see Table 7.1). A non-frequency dependent signal in thermodynamic temperatures corresponds to $\beta=-0.17$ for fits to correlation functions at the DMR frequencies in units of antenna temperature squared. Thus the frequency dependence of the signal in the correlation functions is consistent with the frequency independence of CMB anisotropies.

Figures 7.3 and 7.4 are polar projections of unsmoothed weighted combinations of 31 , 53 and $90 \mathrm{GHz}$ maps. Figure 7.3 is the $A+B$ version (signal + noise) while Figure 7.4 is the A-B (noise) version. Structure is easily visible in Figure 7.3 but not in Figure 7.4. These unsmoothed maps can be compared to the smoothed $53 \mathrm{~A}+\mathrm{B}$ map in Figure 1.4..

Figure 7.5 displays the $A+B$ and $A-B$ versions of the data combined and correlated in three different ways. The presence of the signal in the $A+B$ versions and its absence in the A-B versions is obvious. These combinations are not independent but do show that the correlation function signal is not strongly dependent on how maps are combined and correlated. 

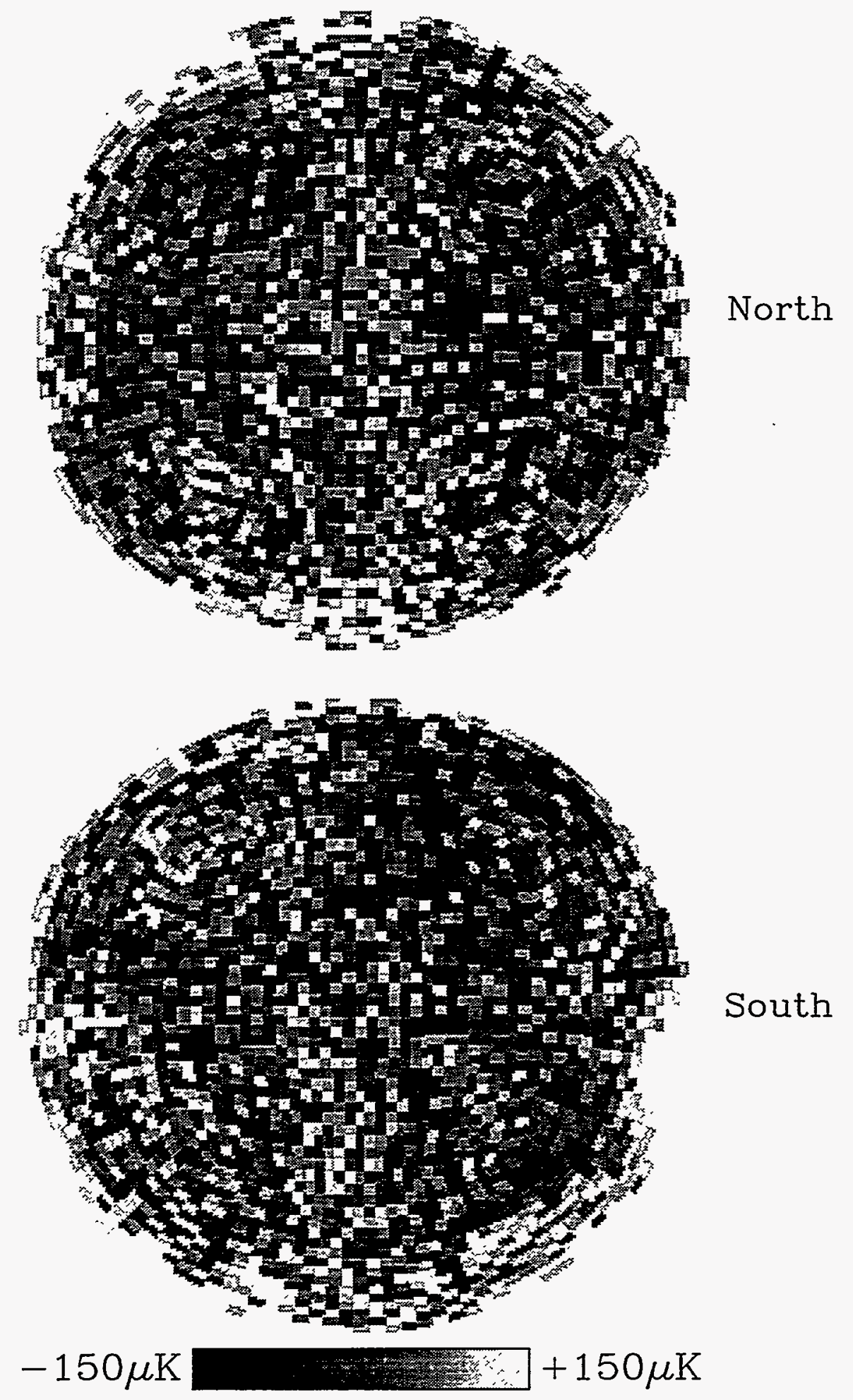

South

Figure 7.3: 2 Yr A+B Sky Maps in Polar Projection. The northern Galactic hemisphere is on the top, southern Galactic hemisphere is on the bottom. They touch at the Galactic center. The Galactic longitude $\ell=90^{\circ}$ is at $90^{\prime}$ clock. The map is an unsmoothed weighted combination of 31,53 and $90 \mathrm{GHz} \mathrm{A}+\mathrm{B}$ maps and thus contains signal and noise. It can be compared with the A-B (noise only) version in Figure 7.4 


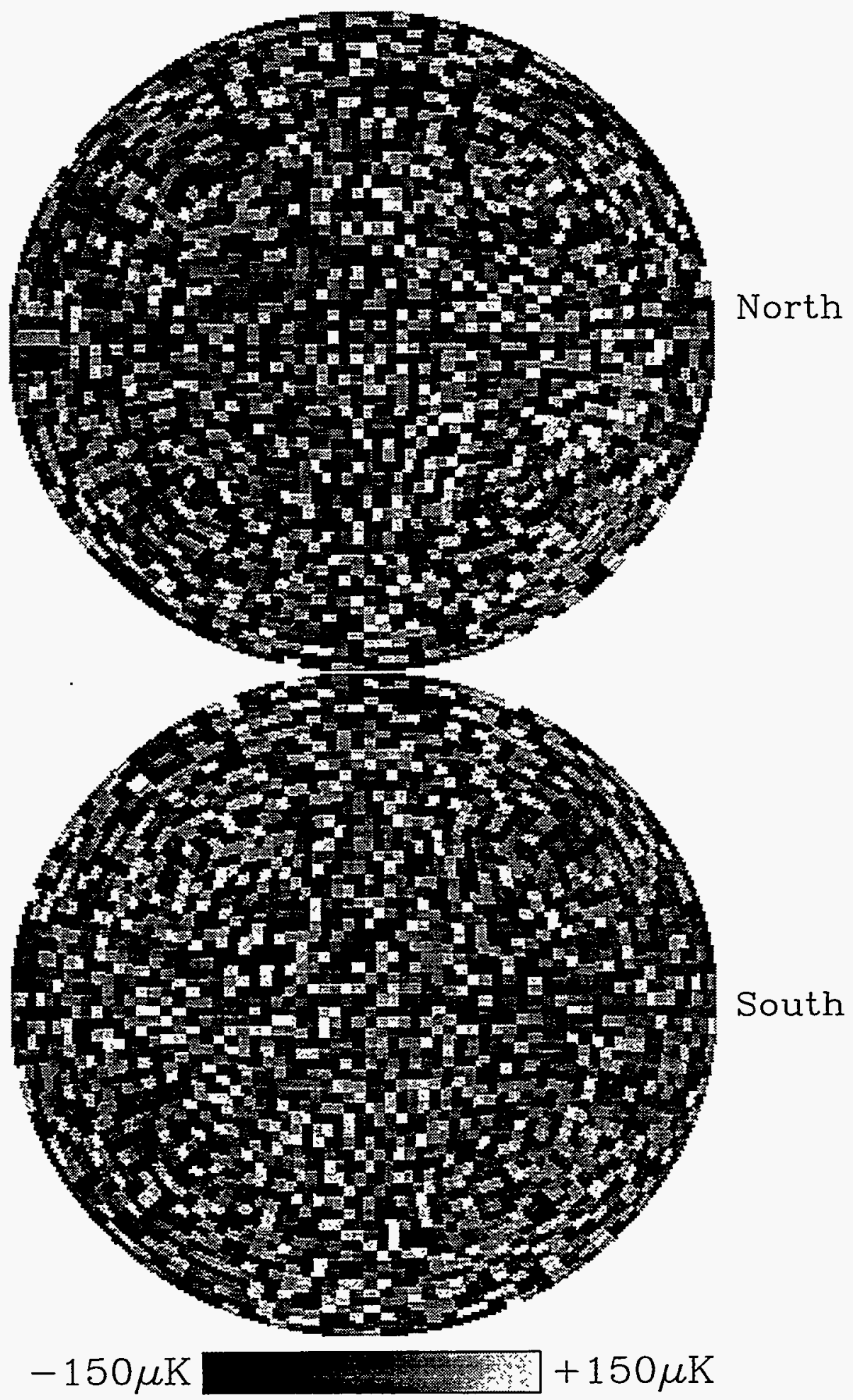

Figure 7.4: 2Yr A-B Sky Map in Polar Projection. The northern Galactic hemisphere is on the top, southern Galactic hemisphere is on the bottom (same orientation as Figure 7.3). The map is an unsmoothed weighted combination of 31,53 and $90 \mathrm{GHz} \mathrm{A}-\mathrm{B}$. 


\section{Best Combinations 2Yr}

$\mathrm{A}+\mathrm{B}$
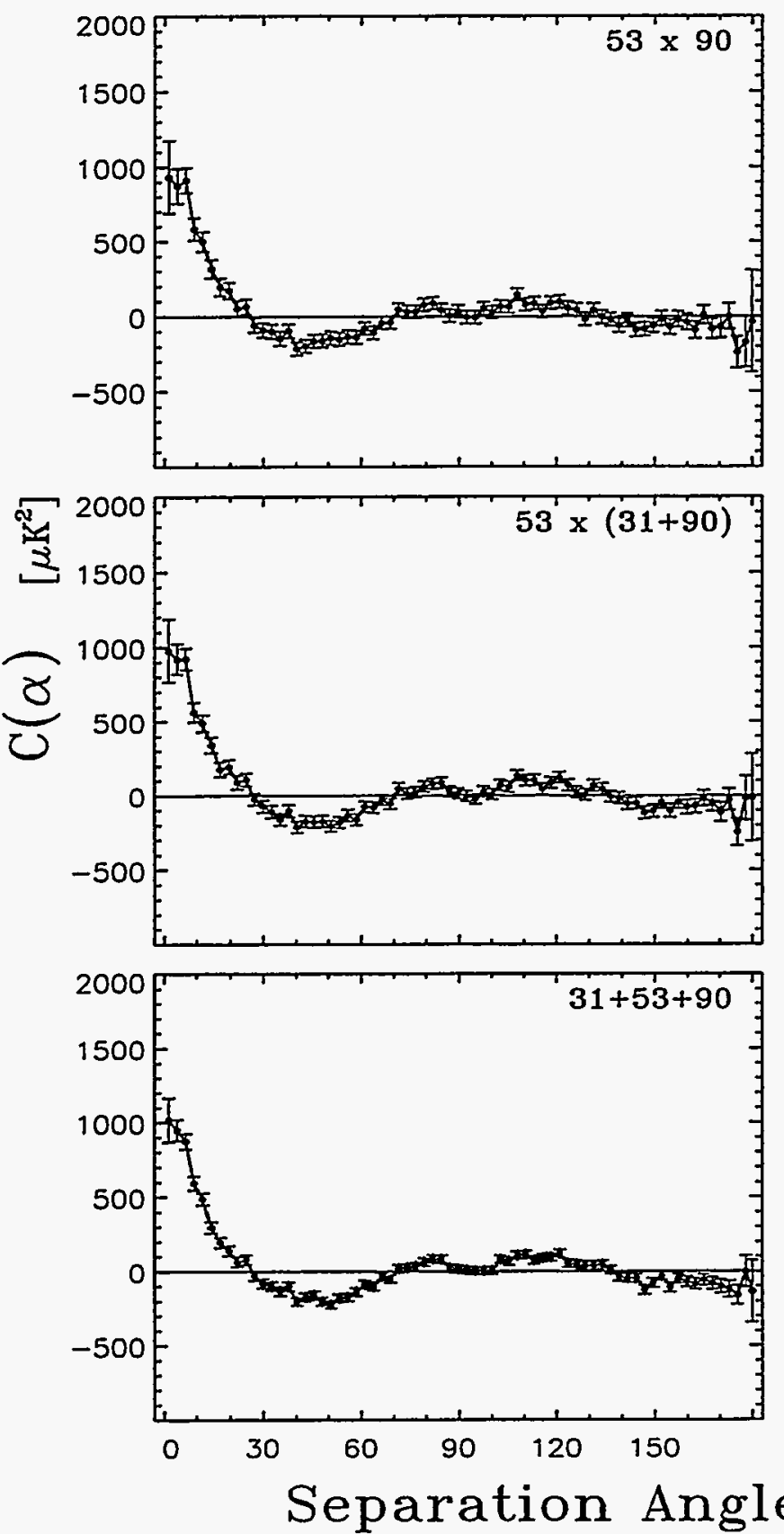

$A-B$
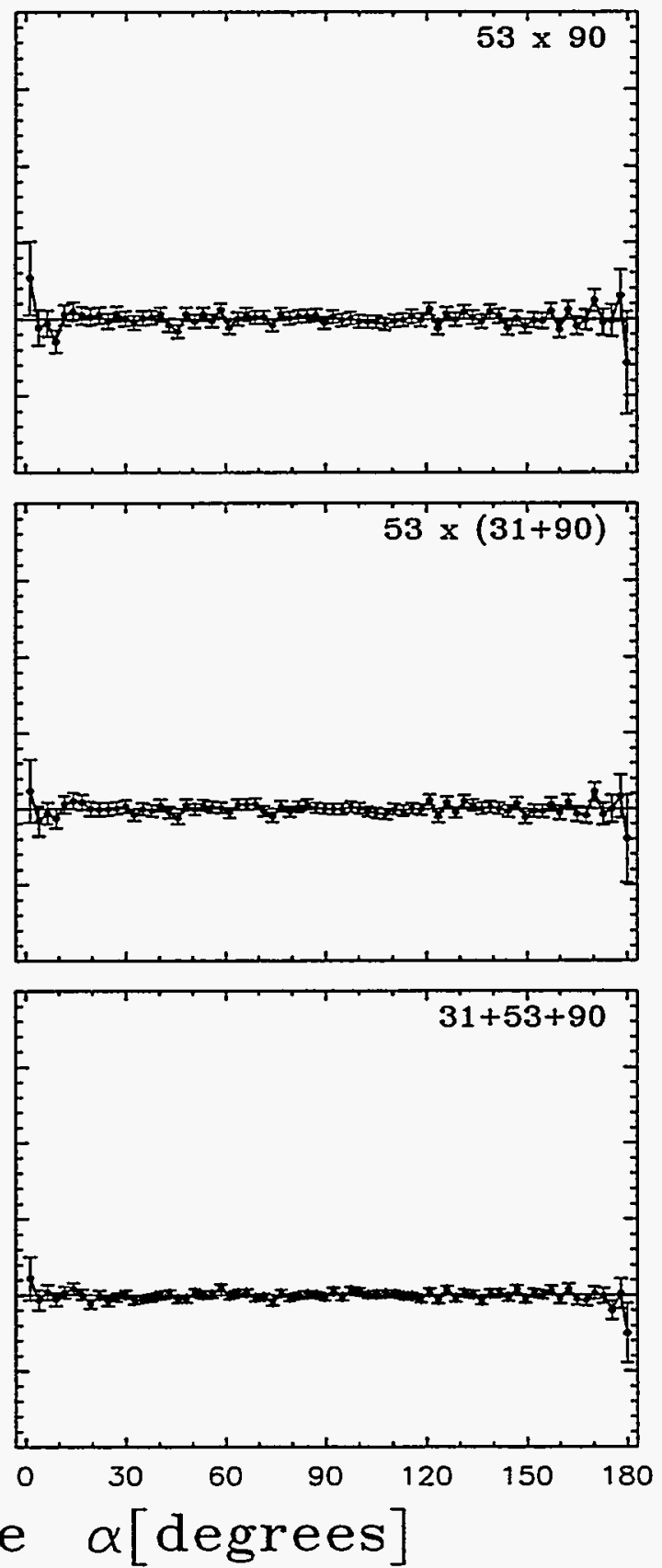

Figure 7.5: 2Yr Best Combinations, $A+B$ and $A-B$ versions. These combinations are not independent but do show that the correlation function signal is not strongly dependent on how maps are combined and correlated. These correlations were computed in the region $|b|>20^{\circ}$ and have had the best fit mean, dipole and quadrupole removed. All plots are cross-correlations except for the auto-correlations in the bottom panels. 


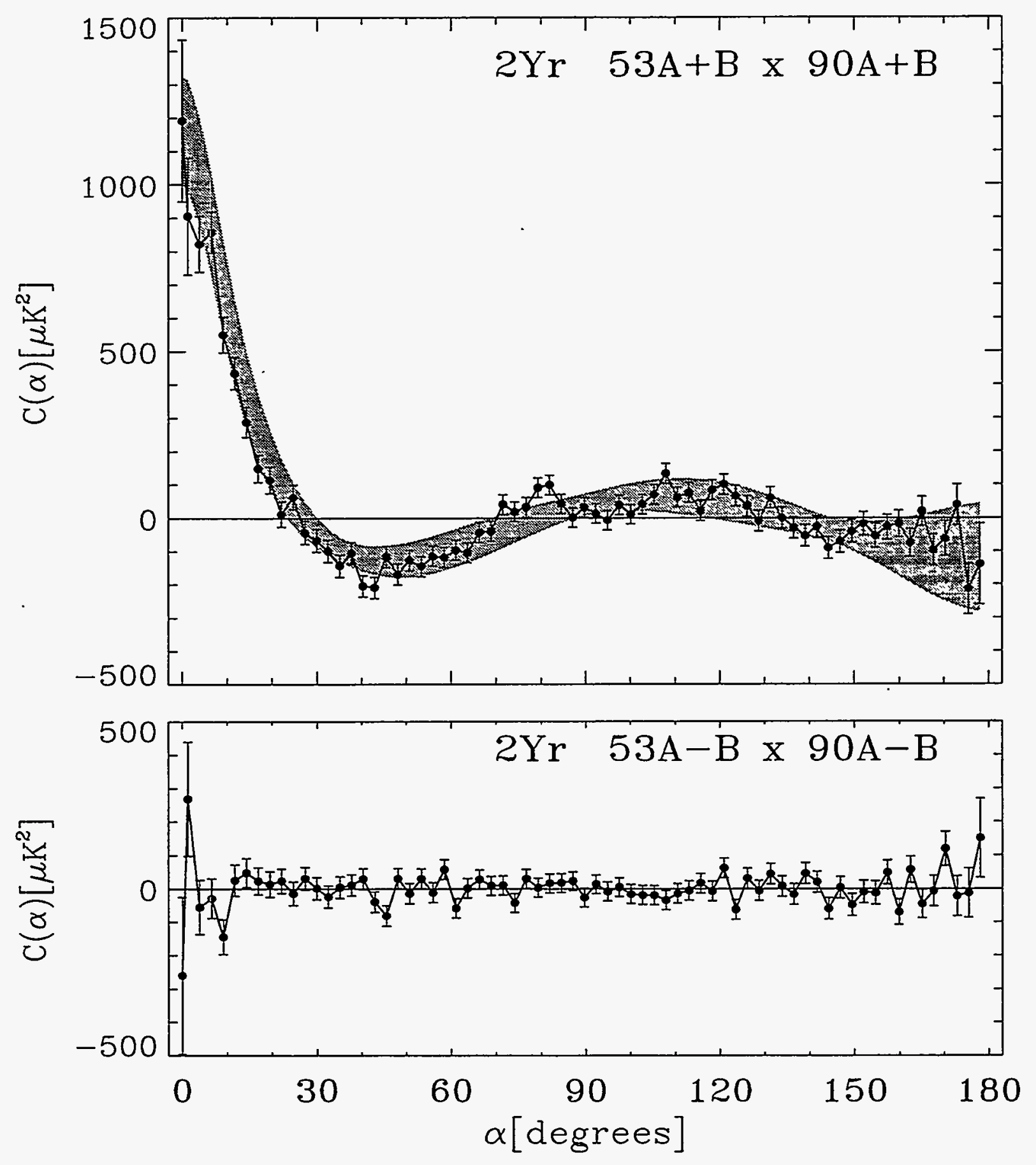

Figure 7.6: 2Yr Cross-correlation of $53 \mathrm{GHz}$ with $90 \mathrm{GHz}$ plus the correlation function for the best fit $n=1.2, Q_{r m s}-P S=17.3 \mu \mathrm{K}$ spectrum: the gray band indicates $68 \%$ confidence levels from cosmic variance. These cross-correlations were computed in the region $|b|>20^{\circ}$ and have had the best fit mean, dipole and quadrupole removed. The $\chi^{2}$ for each of these curves is given in Table 7.2. 
Table 7.1: Frequency Dependence of Signal

\begin{tabular}{|c|c|}
\hline data & $\beta^{a}$ \\
\hline Yr1 & $-0.6 \pm 0.9$ \\
Yr2 & $-0.1 \pm 0.4$ \\
$2 \mathrm{Yr}$ & $-0.1 \pm 0.5$ \\
Ideal $\mathrm{CMB}^{b}$ & -0.17 \\
\hline
\end{tabular}

${ }^{a}$ From fits of $C \propto \nu^{2 \beta}$ to the small $\alpha$ positive bins of the correlation functions of $\mathrm{A}+\mathrm{B}$ maps at the three DMR frequencies.

${ }^{b}$ No frequency dependence yields $\beta=-0.17$ when correlation functions are in units of antenna temperature squared.

Figure 7.6 is the $2 \mathrm{Yr} 53 \mathrm{~A}+\mathrm{B} \times 90 \mathrm{~A}+\mathrm{B}$ cross-correlation function. It is a weighted correlation function for $|b|>20^{\circ}$ with the best fit mean, dipole and quadrupole subtracted. The error bars are noise only and the grey band is the $68 \%$ confidence region defined by the cosmic variance of the best $\chi^{2}$ fit power spectrum parameters $n=1.2, Q_{r m s-P S}=17.3$ $\mu \mathrm{K}$ (see Section 10.2.2).

\subsection{Galactic Latitude Independence}

If the observed signal were produced by some combination of synchrotron, dust and freefree emission from our Galaxy we would expect to find a Galactic latitude dependent signal varying strongly with the frequency of the radiation. Figure 7.7 displays cross-correlations for $53 \mathrm{~A}+\mathrm{B} \times 90 \mathrm{~A}+\mathrm{B}$. The similarity of the $20^{\circ}, 30^{\circ}$ and $40^{\circ}$ cuts in the $\alpha<20^{\circ}$ region is evidence for the Galactic independence of the signal. The $\chi^{2}$ 's of these curves (Section 8.3) are a measure of Galactic latitude independence (and the statistical significance of structure detection) and are tabulated as a function of Galactic cut in Table 7.2.

Figure 7.8 shows the correlation function of the Galactic plane in the $|b|<20^{\circ}$ region for 31,53 and $90 \mathrm{GHz} 2 \mathrm{Yr}$ maps. Notice the strong frequency dependence (logarithmic $\mathrm{y}$-axis!). $\mathrm{C}(0)$ at $31 \mathrm{GHz}$ is ten times as large as $\mathrm{C}(0)$ at $53 \mathrm{GHz}$. This behaviour is not expected of a CMB signal and it is not what is seen in Figure 7.2.

We can use the correlation function of the Galaxy to quantify the frequency dependence of Galactic emission between any two frequencies. Since $C(\alpha) \propto T^{2}$ we can define an 
Table 7.2: Statistical Significance as a Function of Frequency

\begin{tabular}{|rc|cc|ccc|}
\hline & & \multicolumn{2}{|c|}{$A-B$} & \multicolumn{3}{|c|}{$A+B$} \\
\hline Data & Galcut & $\chi^{2}$ & $\chi^{2} / D O F^{a}$ & $\chi^{2}$ & $\chi^{2} / D O F$ & Significance $^{b}$ \\
\hline 31 & $20^{\circ}$ & 62 & 0.89 & 171 & 2.5 & 10.1 \\
53 & $20^{\circ}$ & 114 & 1.6 & 1248 & 17.8 & 34.3 \\
90 & $20^{\circ}$ & 128 & 1.8 & 348 & 5.0 & 16.7 \\
\hline $53 \times 90$ & $10^{\circ}$ & 60 & 0.84 & 4350 & 61.3 & 65.4 \\
& $20^{\circ}$ & 74 & 1.0 & 947 & 13.3 & 29.6 \\
& $30^{\circ}$ & 65 & 0.9 & 576 & 8.1 & 22.5 \\
& $40^{\circ}$ & 85 & 1.2 & 408 & 5.7 & 18.4 \\
\hline
\end{tabular}

a The number of degrees of freedom DOF is equal to the number of correlation function bins: 71 for cross-correlations and 70 for auto-correlations.

${ }^{b}$ Statistical significance in units of noise-only standard deviations is $\sqrt{\chi^{2}-D O F}$, see equation (8.1).

exponent $\beta_{\nu_{1} / \nu_{2}}$ by the equation

$$
\frac{C_{\nu_{1}}(\alpha)}{C_{\nu_{2}}(\alpha)} \equiv\left(\frac{\nu_{1}}{\nu_{2}}\right)^{2 \beta_{\nu_{1} / \nu_{2}}}
$$

Values for $\beta_{\nu_{1} / \nu_{2}}$ are plotted in the lower panel of Figure 7.8. The exponent $\beta_{31 / 53} \approx-2.3$ suggests that free-free emission (with some synchrotron) is predominantly responsible for Galactic plane emission at these frequencies.

\subsection{RMS Temperature Fluctuations and $Q_{r m s}$}

The rms temperature fluctuations are a subset of the correlation function results. We use the fact pointed out in Section 4.7, that the rms temperature fluctuations $\sigma_{s k y}=\sqrt{C(0)}$. When $C(0)$ is from a cross-correlation function the $\sigma_{s k y}$ can be called the cross rms values. Frequency dependent $\sigma_{s k y}$ values have also been computed (Smoot et al.1992, Bennett et al. 1994) using

$$
\sigma_{s k y}=\sqrt{\sigma_{A+B}^{2}-\sigma_{A-B}^{2}}
$$

When the weighting used to compute $\sigma_{A+B}^{2}$ and $\sigma_{A-B}^{2}$ is the same as used to compute the cross-correlation $\mathrm{C}(0)$ of channel $A$ with channel $B$ and when multipole subtraction is 


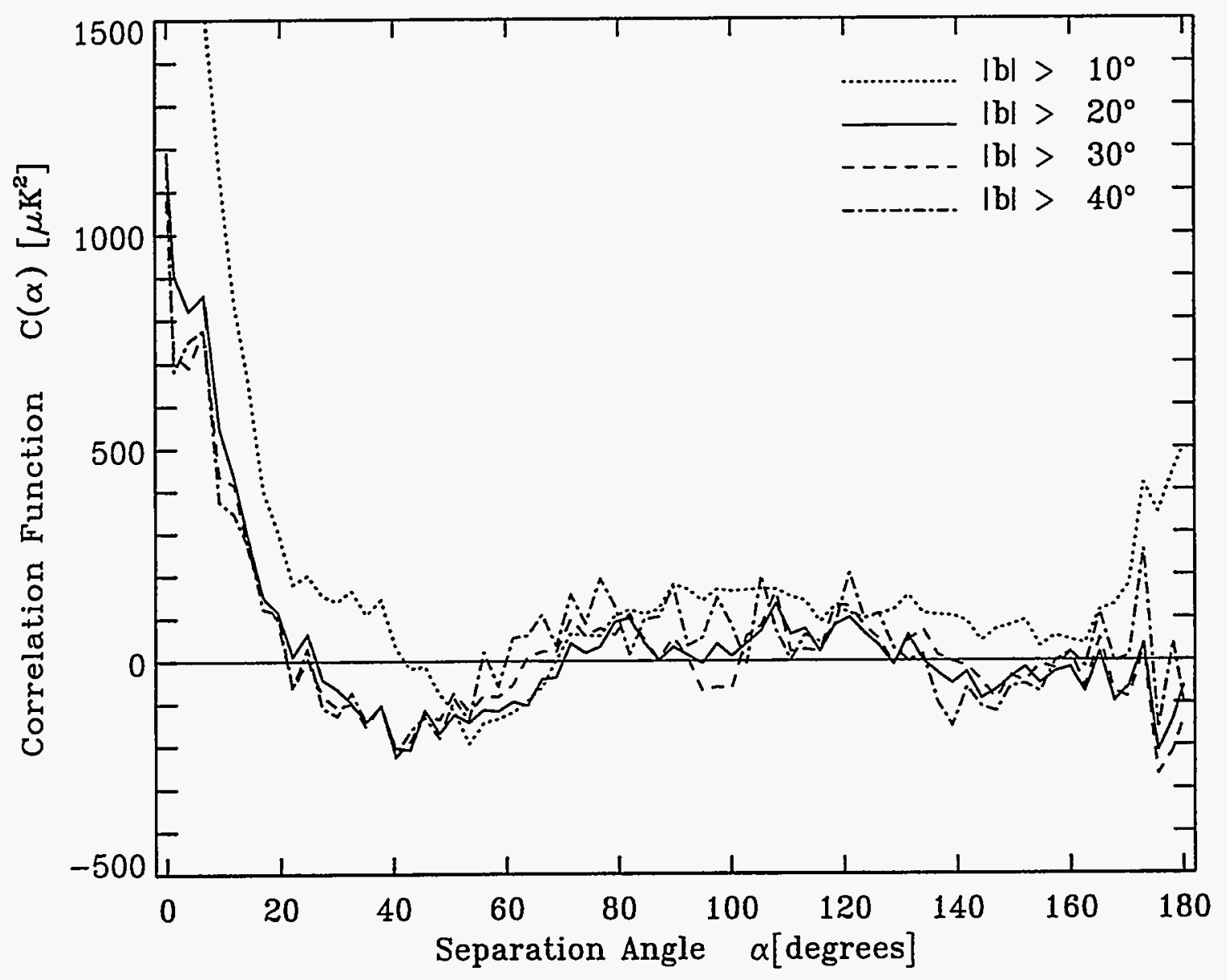

Figure 7.7: 2Yr Galactic Latitude Cut Independence. Cross-correlations of $53 \mathrm{GHz} \mathrm{A}+\mathrm{B}$ with $90 \mathrm{GHz} \mathrm{A}+\mathrm{B}$. For all cases, the best-fit mean, dipole and quadrupole to the region $|b|>20^{\circ}$ was removed. Notice that the Galactic cut independence of the signal is even more pronounced than in the corresponding plot of $\mathrm{Yr}_{\mathrm{r}} 1$ data (Figure 5.3). The $\chi^{2}$ for each of these curves is given in Table 7.2. 

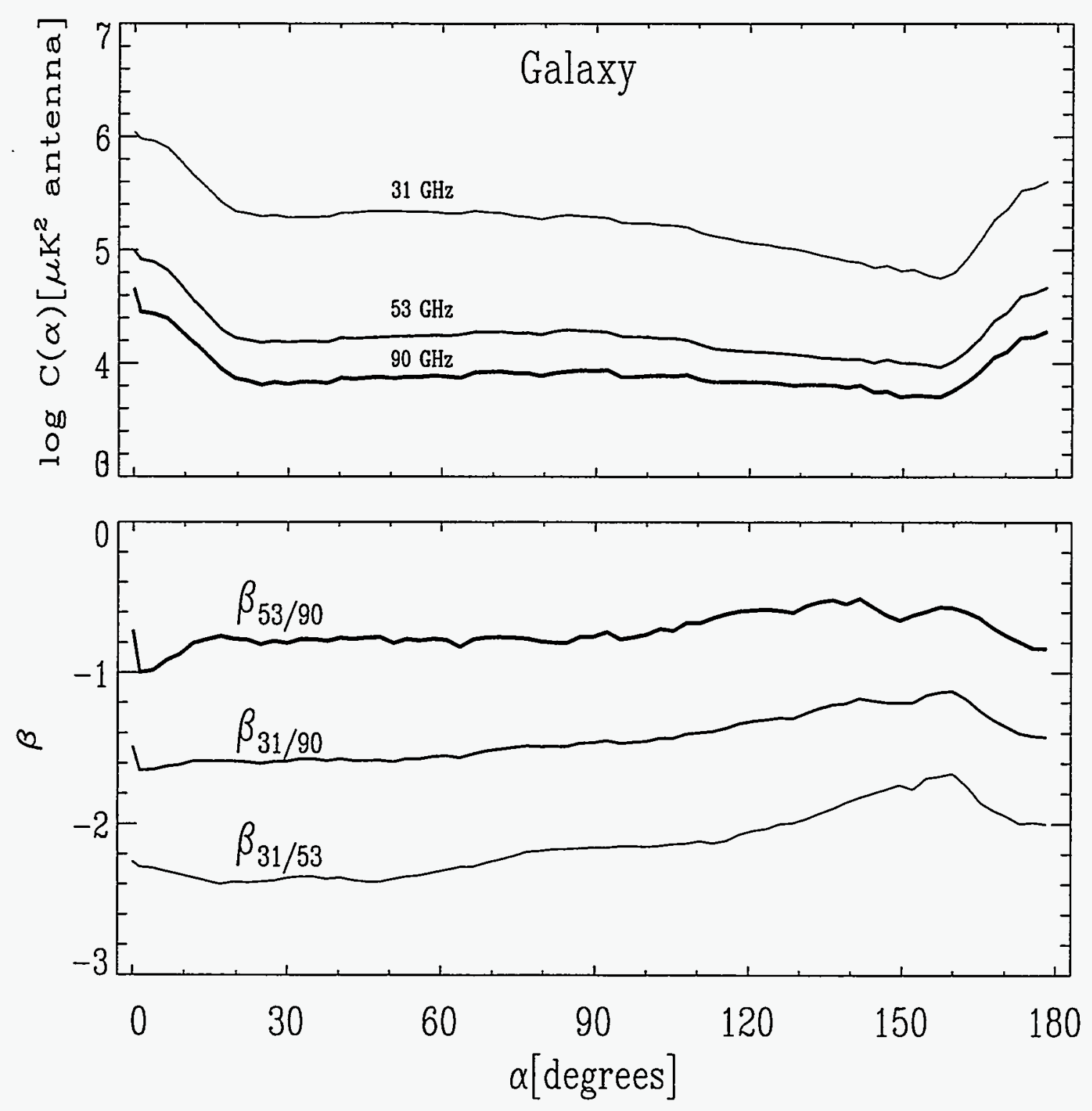

Figure 7.8: Frequency dependence of the Galactic plane of the $A+B$ maps for $|b|<20^{\circ}$. In the top panel the thin, medium and thick lines are 31,53 and $90 \mathrm{GHz}$ respectively. No multipole subtraction has been performed. In the top panel, if a $10^{\circ}$ cut is used instead of $20^{\circ}$, the correlation functions are higher, for $30^{\circ}$ they are lower. The amplitude of the $31 \mathrm{GHz}$ curve is 10 times larger than the $53 \mathrm{GHz}$. The $\beta$ values shown in the bottom panel are related to the spectral shape of Galactic emission (equation (7.2)). The $\beta_{31 / 53} \approx-2.3$ suggests that free-free emission (with some synchrotron) dominates Galactic emission between 31 and $53 \mathrm{GHz}$. 
Table 7.3: RMS Temperature Fluctuations and $Q_{r m s}$ Estimates from $\sqrt{C(0)}^{a}$

\begin{tabular}{|c|c|c|c|c|c|c|c|}
\hline & & Un & weighted ${ }^{b} C$ & $(\alpha)$ & & eighted ${ }^{c} C$ & \\
\hline Data & $b_{\text {cut }}$ & ${\sqrt{C_{m d}(0)}}^{d}$ & $\sqrt{C_{m}}$ & $Q_{r m}^{f}$ & $\sqrt{C_{m d}(0)}$ & $\sqrt{C_{m d q}(0)}$ & $Q_{r}$ \\
\hline & $\left({ }^{\circ}\right)$ & $(\mu K)$ & $(\mu K)$ & $(\mu K)$ & $(\mu K)$ & $(\mu K)$ & 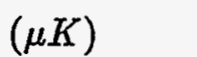 \\
\hline $53 \times 90 \mathrm{Yr} 1$ & 10 & $40.0 \pm 5.1$ & $37.6 \pm 5.4$ & $13.7 \pm 6.8$ & $49.3 \pm 4.3$ & $48.9 \pm 4.3$ & $5.8 \pm 16.8$ \\
\hline & 20 & 28.4 & $26.4 \pm 8.6$ & $10.8 \pm 9.6$ & $35.5 \pm 6.4$ & $34.8 \pm 6.6$ & $7.3 \pm 14.5$ \\
\hline & 30 & $25.1 \pm 10.3$ & $22.7 \pm 11.4$ & $11.1 \pm 10.9$ & $31.3 \pm 8.3$ & $30.0 \pm 8.7$ & $9.0 \pm 13.5$ \\
\hline & 40 & $22.1 \pm 13.8$ & $17.0 \pm 17.9$ & $14.3 \pm 9.8$ & $33.4 \pm 9.1$ & $31.7 \pm 9.6$ & $10.9 \pm 13.0$ \\
\hline$\overline{\mathrm{Yr} 2}$ & 10 & $56.8 \pm 3.6$ & $55.4 \pm 3.7$ & $12.6 \pm 7.5$ & $55.7 \pm 3.8$ & $55.3 \pm 3.9$ & $7.1 \pm 14.0$ \\
\hline & 20 & $45.6 \pm 5.0$ & $45.1 \pm 5.1$ & $7.1 \pm 14.8$ & $39.9 \pm 5.8$ & $39.7 \pm 5.8$ & $4.5 \pm 24.0$ \\
\hline & 30 & $42.7 \pm 6.1$ & $41.8 \pm 6.2$ & $8.6 \pm 13.7$ & $39.6 \pm 6.6$ & $39.2 \pm 6.6$ & $6.0 \pm 20.1$ \\
\hline & 40 & $43.0 \pm 7.1$ & $42.3 \pm 7.2$ & $7.7 \pm 18.0$ & $39.0 \pm 7.8$ & $38.5 \pm 7.8$ & $6.7 \pm 20.8$ \\
\hline $2 \mathrm{Yr}_{\mathrm{r}}$ & 10 & $46.7 \pm 3.2$ & $44.8 \pm 3.3$ & $13.3 \pm 5.1$ & $50.4 \pm 2.2$ & $50.0 \pm 2.2$ & $6.7 \pm 7.6$ \\
\hline & 20 & $35.7 \pm 4.6^{h}$ & $34.4 \pm 4.7$ & $9.3 \pm 8.0$ & $35.1^{i} \pm 3.3$ & $34.5 \pm 3.4$ & $6.3 \pm 8.5$ \\
\hline & 30 & $33.5 \pm 5.6$ & $31.9 \pm 5.8$ & $10.4 \pm 8.2$ & $33.9 \pm 3.9$ & $32.9 \pm 4.1$ & $8.3 \pm 7.5$ \\
\hline & 40 & $35.4 \pm 6.2$ & $33.5 \pm 6.5$ & $11.8 \pm 8.6$ & $35.3 \pm 4.4$ & $34.0 \pm 4.6$ & $9.6 \pm 7.5$ \\
\hline $53 A \times B^{j}$ Yr1 & & $53.0 \pm 4.1$ & $50.5 \pm 4.3$ & $16.4 \pm 6.1$ & $56.7 \pm 4.7$ & $55.3 \pm 4.8$ & $12.8 \pm 9.6$ \\
\hline & 20 & $45.2 \pm 5.4$ & $44.0 \pm 5.5$ & $10.5 \pm 10.7$ & $40.1 \pm 7.2$ & $39.5 \pm 7.3$ & $7.3 \pm 18.5$ \\
\hline & 30 & $47.0 \pm 5.9$ & $46.5 \pm 6.0$ & $7.0 \pm 18.1$ & $40.3 \pm 8.2$ & $39.8 \pm 8.3$ & $6.4 \pm 24.1$ \\
\hline & 40 & $45.9 \pm 7.2$ & $44.4 \pm 7.4$ & $11.9 \pm 12.9$ & $37.7 \pm 10.3$ & $36.1 \pm 10.8$ & $11.1 \pm 16.2$ \\
\hline Yr2 & 10 & $64.6 \pm 3.4$ & $62.3 \pm 3.5$ & $17.3 \pm 5.8$ & $60.4 \pm 4.5$ & $59.9 \pm 4.5$ & $7.7 \pm 16.3$ \\
\hline & 20 & $58.9 \pm 4.1$ & $57.9 \pm 4.2$ & $11.2 \pm 10.0$ & $49.3 \pm 6.0$ & $48.9 \pm 6.0$ & $6.2 \pm 22.0$ \\
\hline & 30 & $54.2 \pm 5.1$ & $53.4 \pm 5.1$ & $9.7 \pm 13.0$ & $46.4 \pm 7.2$ & $45.7 \pm 7.3$ & $8.3 \pm 18.7$ \\
\hline & 40 & $53.8 \pm 6.0$ & $52.3 \pm 6.1$ & $12.8 \pm 11.5$ & $44.1 \pm 8.8$ & $42.5 \pm 9.1$ & $11.8 \pm 15.1$ \\
\hline $2 \mathrm{Yr}$ & 10 & $55.2 \pm 2.8$ & $52.6 \pm 3.0$ & $16.9 \pm 4.3$ & $54.7 \pm 2.5$ & $53.6 \pm 2.6$ & $11.0 \pm 6.0$ \\
\hline & 20 & $44.4 \pm 3.9$ & $43.0 \pm 4.0$ & $11.2 \pm 7.2$ & $38.1 \pm 3.9$ & $37.4 \pm 4.0$ & $7.3 \pm 9.4$ \\
\hline & 30 & $43.9 \pm 4.5$ & $42.8 \pm 4.6$ & $9.6 \pm 9.6$ & $37.1 \pm 4.6$ & $36.3 \pm 4.6$ & $7.4 \pm 10.6$ \\
\hline & 40 & $50.1 \pm 4.6$ & $48.4 \pm 4.8$ & $13.2 \pm 8.1$ & $40.5 \pm 4.8$ & $38.9 \pm 5.0$ & $11.5 \pm 7.9$ \\
\hline
\end{tabular}

${ }^{a} \mathrm{C}(0)$ are cross-correlations with noise-only error bars

${ }^{b}$ Unweighted correlation function and multipole subtraction (see Section 4.6)

c Weighted correlation function and multipole subtraction

${ }^{d}$ best-fit mean and dipole for $|b|>20^{\circ}$ subtracted

${ }^{e}$ best-fit mean, dipole and quadrupole for $|b|>20^{\circ}$ subtracted

$f$ defined by equation 4.29

g $53 A+B \times 90 A+B$

${ }^{h}$ Bennett et al. (1994) value is $36 \pm 5$

$i$ see Figure 7.9

j $53 A \times 53 B$ 
handled identically then the two techniques are equivalent

$$
C(0) \equiv \sigma_{A+B}^{2}-\sigma_{A-B}^{2}
$$

Table 7.3 tabulates cross-correlation results of $53 \times 90$ and $53 \mathrm{~A} \times 53 \mathrm{~B}$ for $\mathrm{Y}_{\mathrm{r}} 1, \mathrm{Yr} 2$ and $2 \mathrm{Yr}$ maps. Two important quantities are computed as a function of Galactic cut: rms temperature fluctuations $\sqrt{C_{m d}(0)}$ and the quadrupole moment of our observable sky $Q_{r m s}$ ( $m d$ denotes that the mean and dipole have been removed, while $m d q$ denotes that in addition the quadrupole has been removed). We find for the two year data set weighted cross-correlation of $53 \mathrm{GHz}$ with $90 \mathrm{GHz} \sqrt{C_{m d}(0)}=35 \pm 4 \mu \mathrm{K}$. This is the weighted average from Galactic latitude cuts of $20^{\circ}, 30^{\circ}$, and $40^{\circ}$ and the error bar is from noise only. The unweighted cross-correlation yields the same value with a larger uncertainty $\sqrt{C_{m d}(0)}=35 \pm 6 \mu \mathrm{K}$.

The relative merits of the various weightings of rms statistics $\left(\sum_{i} T_{i}^{2}, \sum_{i} w_{i} T_{i}^{2}\right.$, $\sum_{i} w_{i}^{2} T_{i}^{2}$ or for two maps $\left.\sum_{i=j} w_{i} w_{j} T_{i} T_{j}\right)$ will not be discussed here except for the remark that the square root of the weighted auto-correlation function at zero lag $\left(\sum_{i} w_{i}^{2} T_{i}^{2}\right)$ minimizes the variance due to the noise in the map pixel temperatures (see Smoot et al. 1994, Bennett et al. 1994).

$Q_{\text {rms }}$ is the actual quadrupole of our horizon as estimated from observations of the high Galactic latitude CMB sky and should not be confused with the power spectrum normalization $Q_{r m s-P S}$. Estimates of the observed quadrupole $Q_{r m s}$ are computed from equation (4.29) and the $\sqrt{C_{m d}(0)}$ and $\sqrt{C_{m d q}(0)}$ values listed in Table 7.3. Weighted averages of the results for Galactic latitude cuts of $20^{\circ}, 30^{\circ}$, and $40^{\circ}$ from the $2 \mathrm{Yr} 53 \mathrm{x}$ 90 weighted correlation function yields $Q_{r m s}=8 \pm 8 \mu \mathrm{K}$ while the unweighted correlation functions yield $Q_{r m s}=10 \pm 8 \mu \mathrm{K}$. Averaging these two yields $Q_{r m s}=9 \pm 8 \mu \mathrm{K}$. 


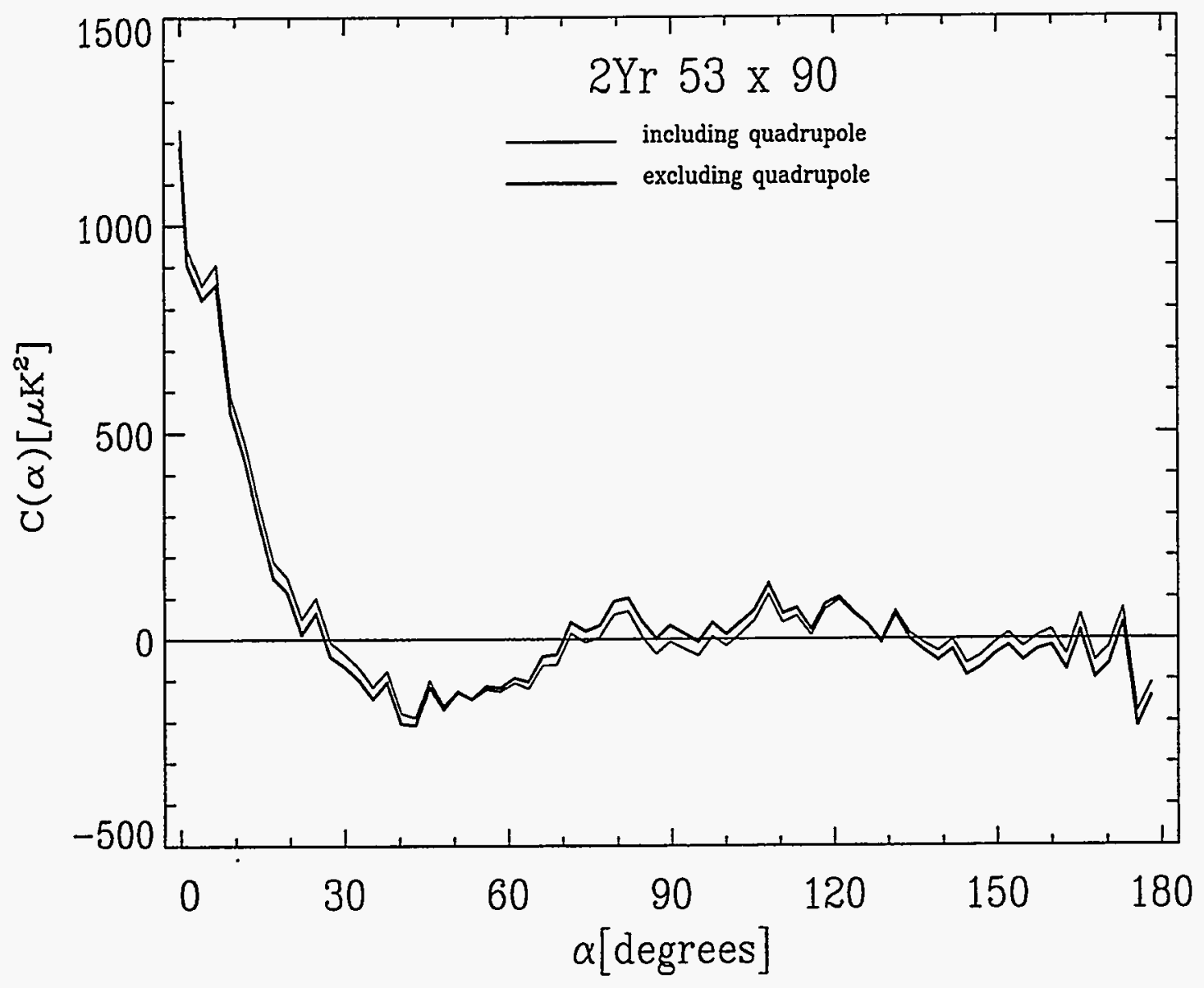

Figure 7.9: 2Yr Correlation Functions with and without quadrupole. Cross-correlation function of $53 \mathrm{GHz}$ with $90 \mathrm{GHz}$ for $|b|>20^{\circ}$. The difference in $\mathrm{C}(0)$ values is used to compute $Q_{r m s}$. 


\title{
Chapter 8
}

\section{Correlation Function Comparison of the First and Second Year Maps}

\begin{abstract}
Confirmation of the DMR first year results can be obtained from other experiments but also from a comparison with the independent second year DMR results. We present a correlation function comparison of the first year (Yr1) and second year (Yr2) data. The Yr1 and Yr2 data sets are reasonably consistent. The positive correlation for separation angles less than $\sim 20^{\circ}$ is robust to Galactic latitude cut and is very stable from year to year. The detected structure in the correlation functions has a statistical significance of $>9 \sigma$ and $>10 \sigma$ for Yr1 and Yr2 respectively. Yr2 has higher rms temperature fluctuations. For example, the weighted $53 \times 90$ correlation functions at zero lag yield $40 \pm 7 \mu \mathrm{K}$ for $\mathrm{Y}_{\mathrm{r}} 2$ and $33 \pm 8 \mu \mathrm{K}$ for $\mathrm{Yr}_{\mathrm{r}} 1$ but the difference is not statistically significant. The cross-correlation of the 53 $\mathrm{GHz}$ and $90 \mathrm{GHz}$ maps yields cross-quadrupole values (Section 4.8) and indicates that the second year data has a lower quadrupole than the first year but the error bars are not small enough to claim inconsistency.
\end{abstract}

\subsection{Year One vs Year Two Data}

In the following correlation function comparison of $\mathrm{Yr} 1$ with $\mathrm{Yr} 2$ we will use only maps from the Pass 2 software processing (see Appendix E). On October 4, 1991 the 31B channel noise increased dramatically and we do not use $31 \mathrm{~B}$ data after this date. A comparison of 
the noise levels in the $\mathrm{Yr}_{1}$ and $\mathrm{Yr} 2$ maps can be obtained by comparing column 4 and 6 of Table 2.1. The percent changes in the rms noise values from Yr1 to $\mathrm{Yr} 2$ in channels 31A, $31 \mathrm{~B}, 53 \mathrm{~A}, 53 \mathrm{~B}, 90 \mathrm{~A}, 90 \mathrm{~B}$ are $-1.8,-1.3,0.0,0.0,-1.5$ and +2.0 respectively.

\subsection{Channel Comparison, $\mathrm{A}+\mathrm{B}$ vs $\mathrm{A}-\mathrm{B}$ Comparison}

The signal seen in the DMR map correlation functions is essentially an increasing positive correlation as the separation angle $\alpha$ decreases. Thus the "signal" is the slope leading up to a $C(0) \sim 1000 \mu \mathrm{K}^{2}$. This signal is apparent in the separate channels for Yr1 and $Y_{r 2}$ which are compared in Figure 8.1. Notice the consistency from year to year in each pair of curves. The correlation functions of channel $90 \mathrm{~A}$ do not seem to show the expected signal. However when the maps are combined the noise fluctuations average out and the result is reasonably consistent with the other channels (see Figure 7.1).

In Figure 8.2 the signal of the $A+B$ correlation functions can be compared with the noise of the A-B correlation functions. Here again the consistency of $\mathrm{Yr} 1$ and $\mathrm{Yr} 2$ is evident by the similarity of the two curves in each panel. The $A+B$ curves for 31,53 and $90 \mathrm{GHz}$ have a slope leading up to $C(0) \sim 1000 \mu \mathrm{K}^{2}$. The $31 \mathrm{GHz} \mathrm{A}+\mathrm{B}$ curve is very noisy and the $20^{\circ}$ galcut is not enough to prevent Galactic synchrotron contamination. All curves are for $|b|>20^{\circ}$. The frequency dependence of the correlation function signal is consistent with a CMB signal and is tabulated in Table 7.1. Notice that 90A-B has an anomalously high $C(0)$ for Yr1 but for Yr2 the value comes down to an acceptable level (compare Figure 7.2).

Figure 8.3 displays the cross-correlation of $53 \mathrm{~A}+\mathrm{B}$ with $90 \mathrm{~A}+\mathrm{B}$ in the top panel and $53 \mathrm{~A}-\mathrm{B}$ with $90 \mathrm{~A}-\mathrm{B}$ in the bottom panel. Correlation functions from $\mathrm{Yr} 1, \mathrm{Yr} 2$ and $2 \mathrm{Yr}$ are superimposed. In this very sensitive test the signal is present in the $A+B$ but not in the A-B traces. The signal from Yr2 is consistent with the signal from Yr1. This consistency is further verified by permutations of these cross-correlations. For example the cross-correlations of $53 A \pm B$ (Yr1) with $90 A \pm B$ (Yr2) and $53 A \pm B$ (Yr2) with $90 A \pm B$ ( $Y r 1$ ) yield results very similar to those seen in Figure 8.3.

The Yr1 and Yr2 maps can be compared in Figures 8.4 and 8.5. The weighted combination of all the channels are displayed using a $\Delta T / T= \pm 10^{-4}$ greyscale. The maps have $2.6^{\circ}$ pixels and have not been smoothed. Away from the Galactic plane, several cool spots are recognizable in both maps. 
Separate Channels 1990 Pass2 vs 1991 Pass2

Channel A
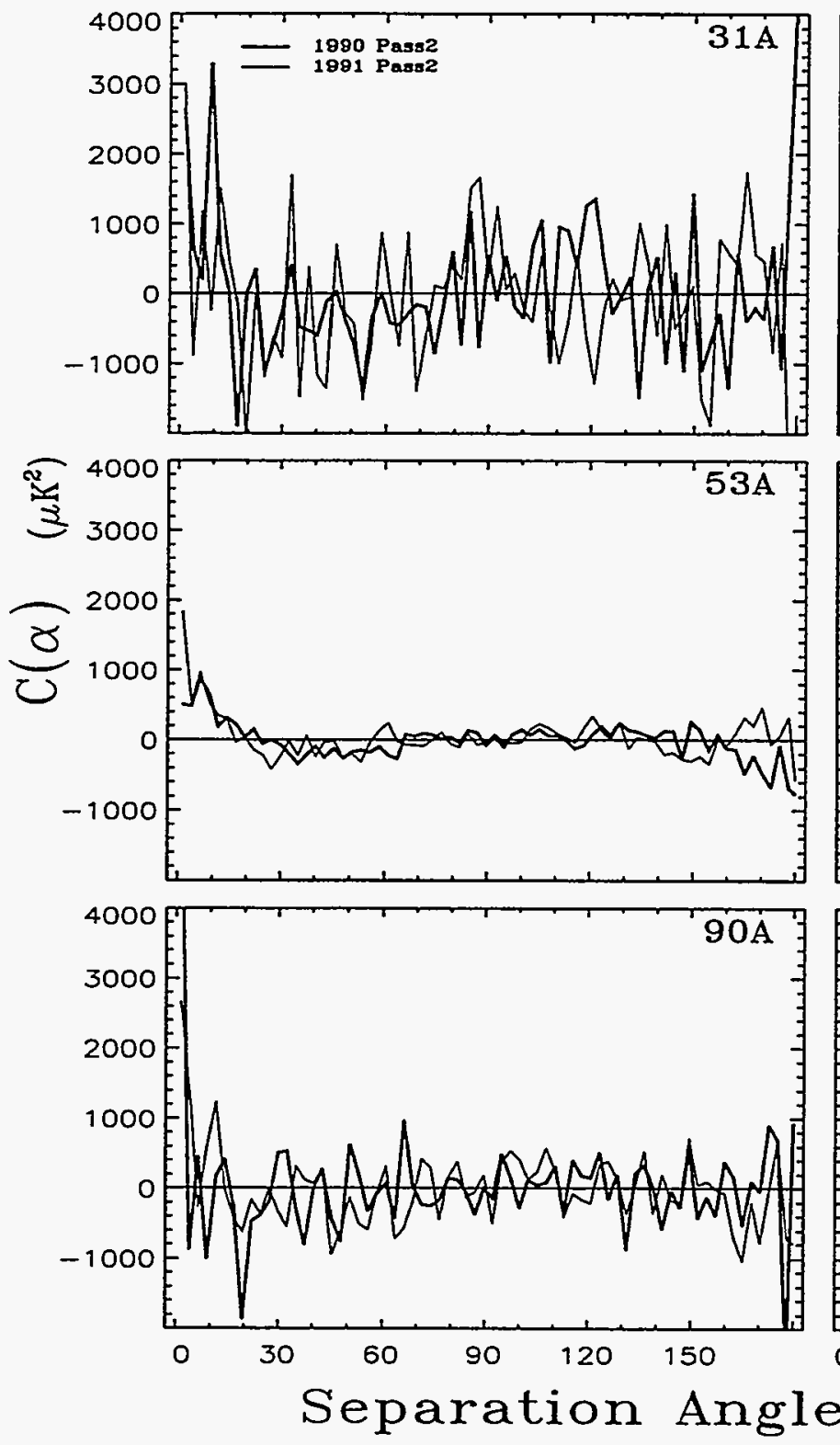

Channel B
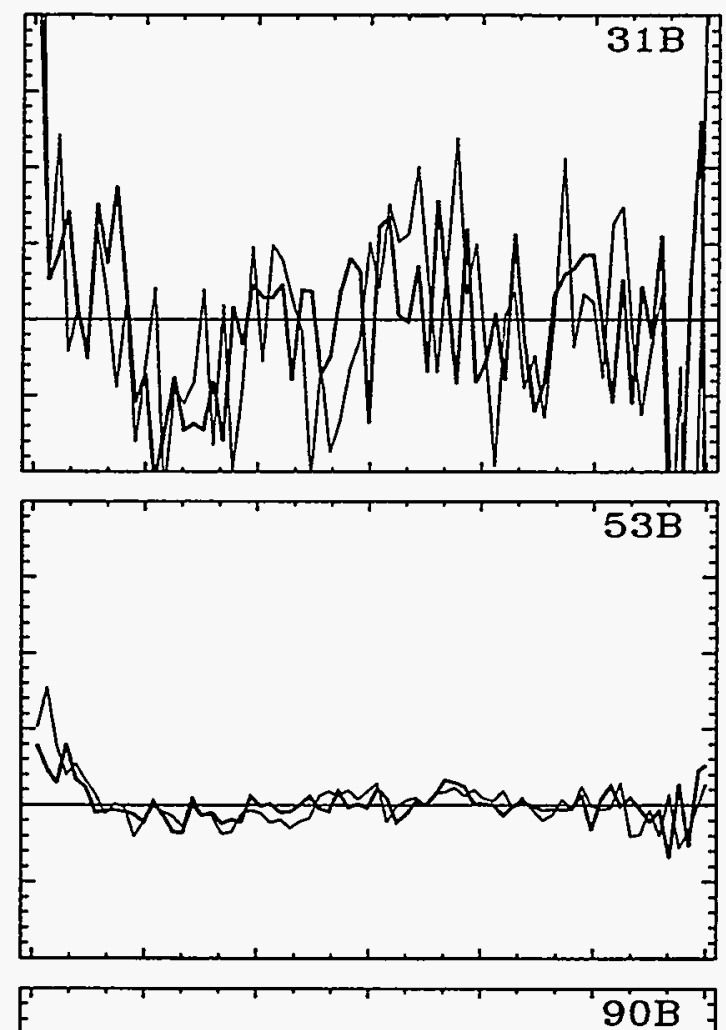

Figure 8.1: Yr1 vs Yr2 Separate Channel Comparison. Notice that the signal (the slope leading up to $C(0) \sim 1000 \mu \mathrm{K}^{2}$ ) can be seen in each of the channels with the exception of the $90 \mathrm{~A}$. The $31 \mathrm{GHz}$ signal is noisy and contaminated by Galactic synchrotron. The signals are reasonably consistent from year to year. All curves are for $|b|>20^{\circ}$ and have had best fit means, dipoles and quadrupoles removed. 
Auto-Correlations 1990 Pass2 vs 1991 Pass2

$A+B$
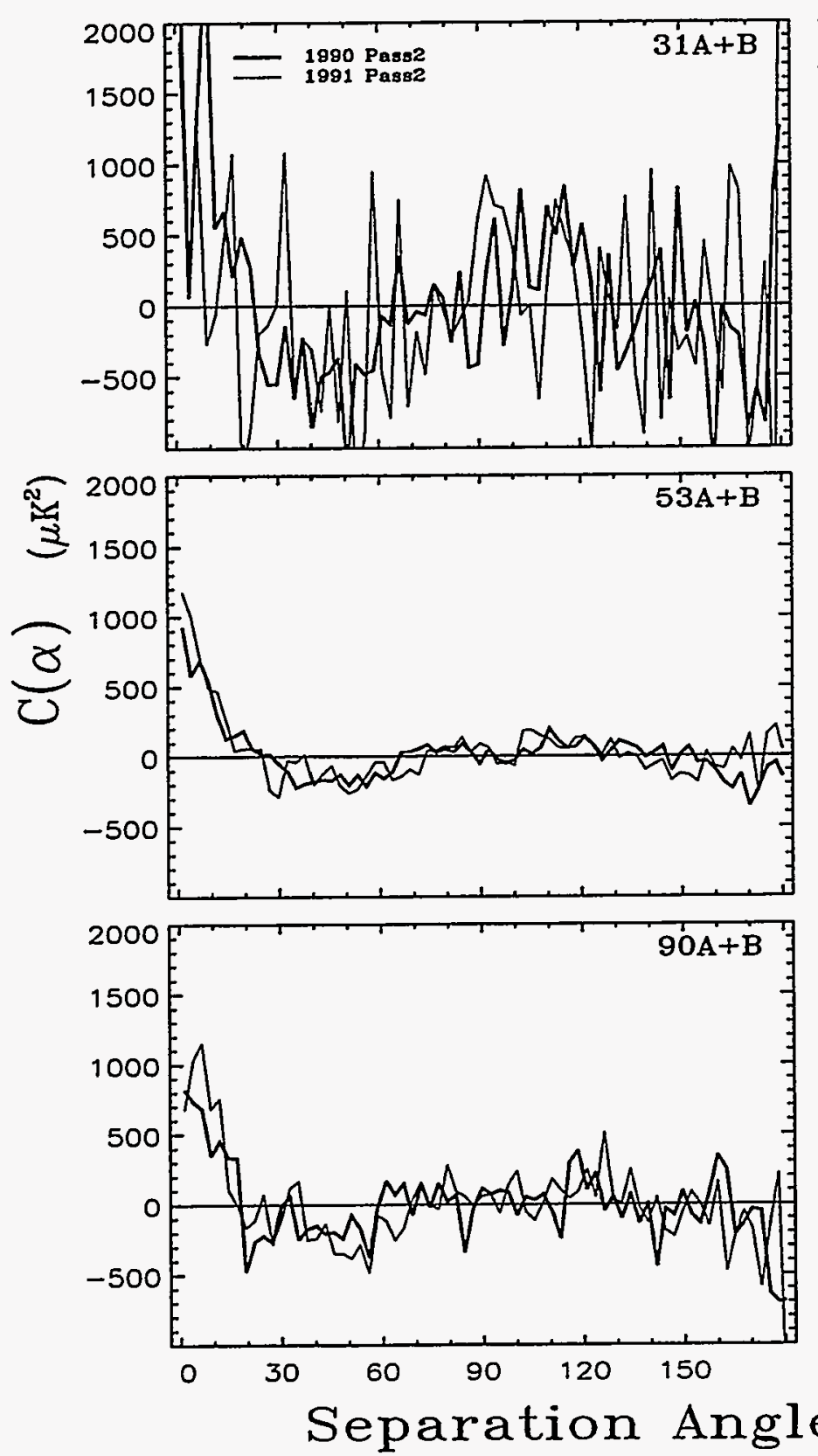

A - B
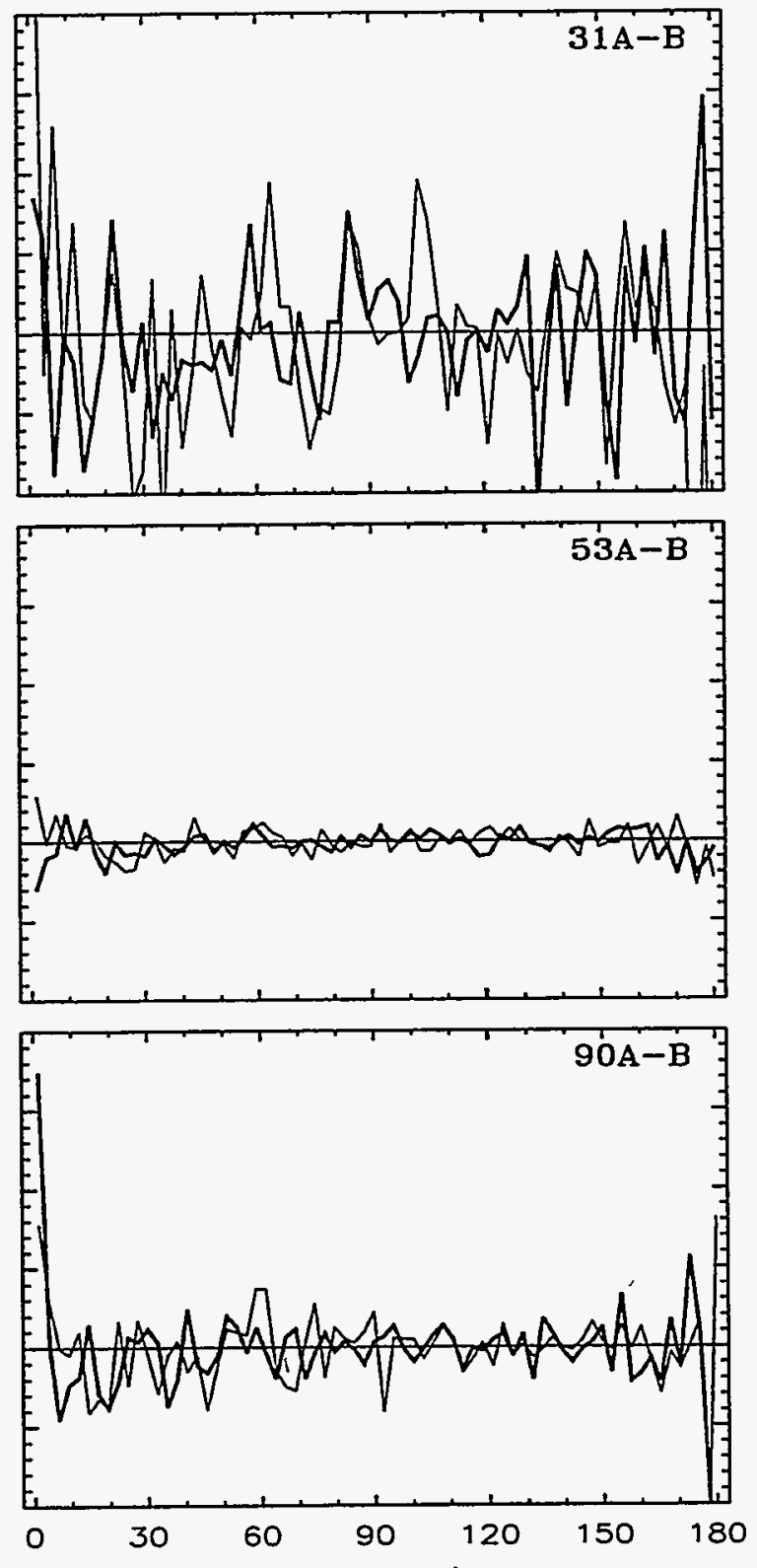

$\alpha$ (degrees)

Figure 8.2: Yr1 vs $\mathrm{Yr}_{\mathrm{r}} \mathrm{A}+\mathrm{B}$ vs $\mathrm{A}-\mathrm{B}$ Comparison. The signal is apparent in the $\mathrm{A}+\mathrm{B}$ correlation functions and absent in the A-B correlation functions. $90 \mathrm{GHz} A-\mathrm{B}$ has an anomalous first bin. All curves are for $|b|>20^{\circ}$ and have had best fit means, dipoles and quadrupoles removed. 

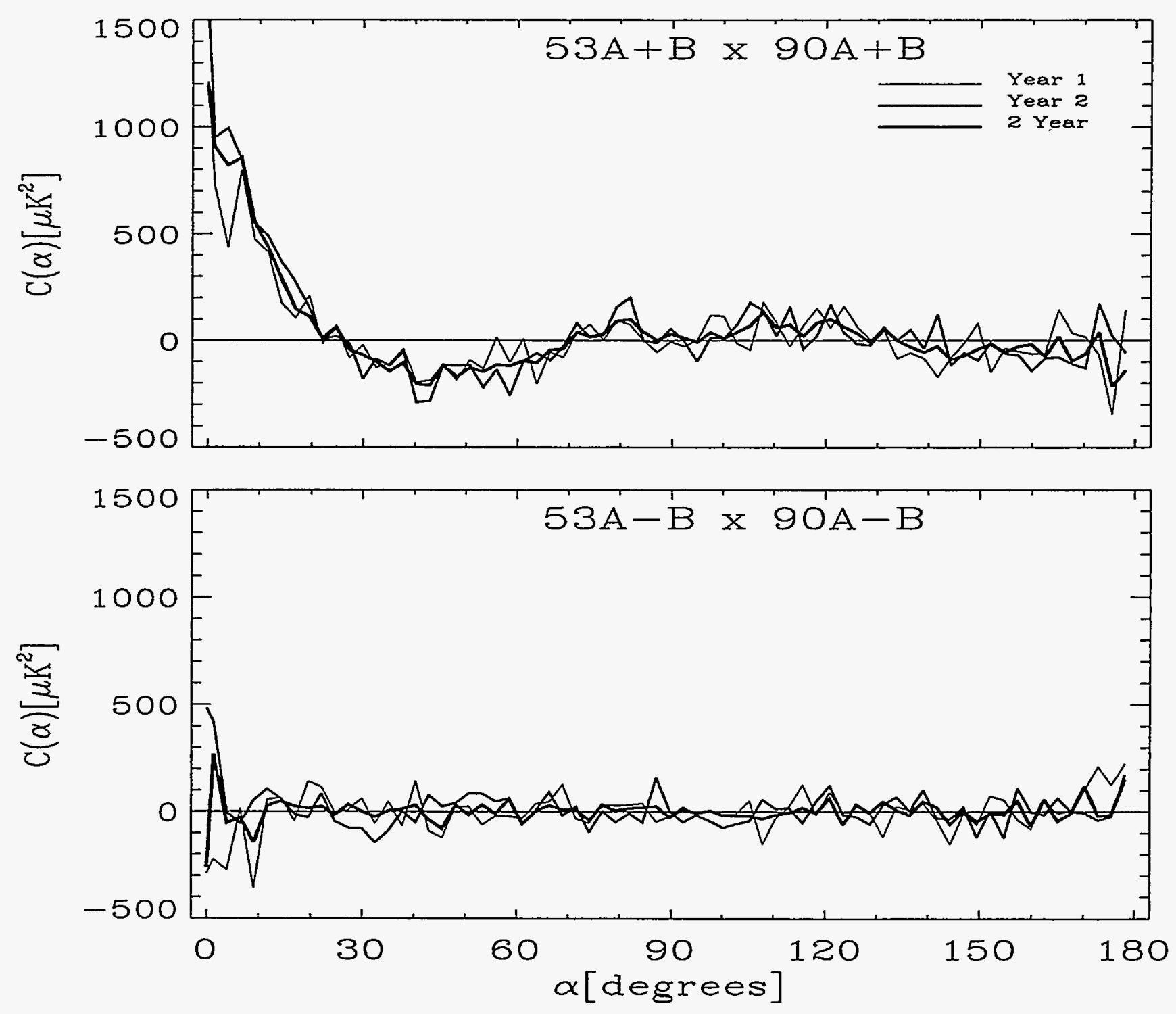

Figure 8.3: Correlation Function Comparison of Yr1, Yr2 and 2Yr. Cross-correlation function of $53 \mathrm{GHz}$ with $90 \mathrm{GHz}$ for $|b|>20^{\circ}$. The best-fit mean, dipole and quadrupole have been removed. Top is for the sum maps, and bottom is for the difference maps. 


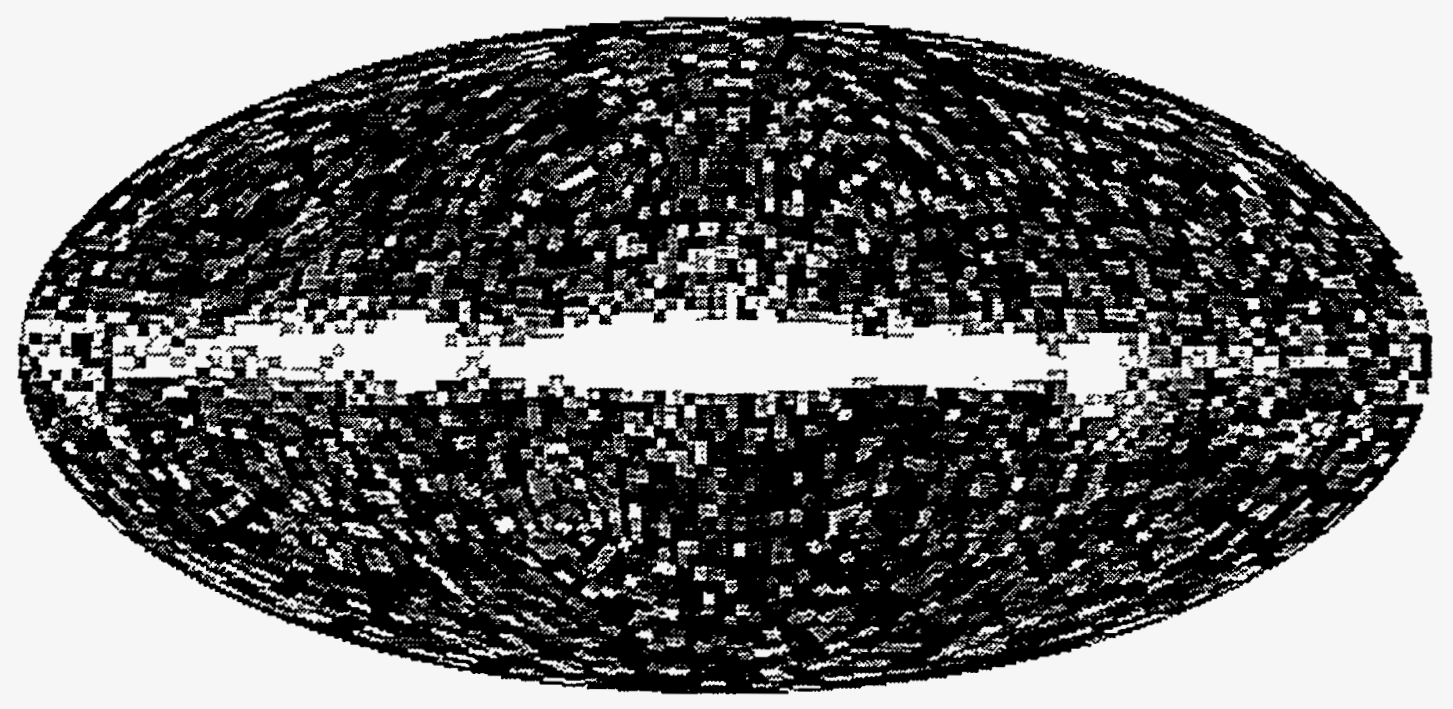

$-270 \mu \mathrm{K}$

Figure 8.4: Yr1 Map. Weighted combination of $A+B$ 31, 53 and $90 \mathrm{GHz}$.

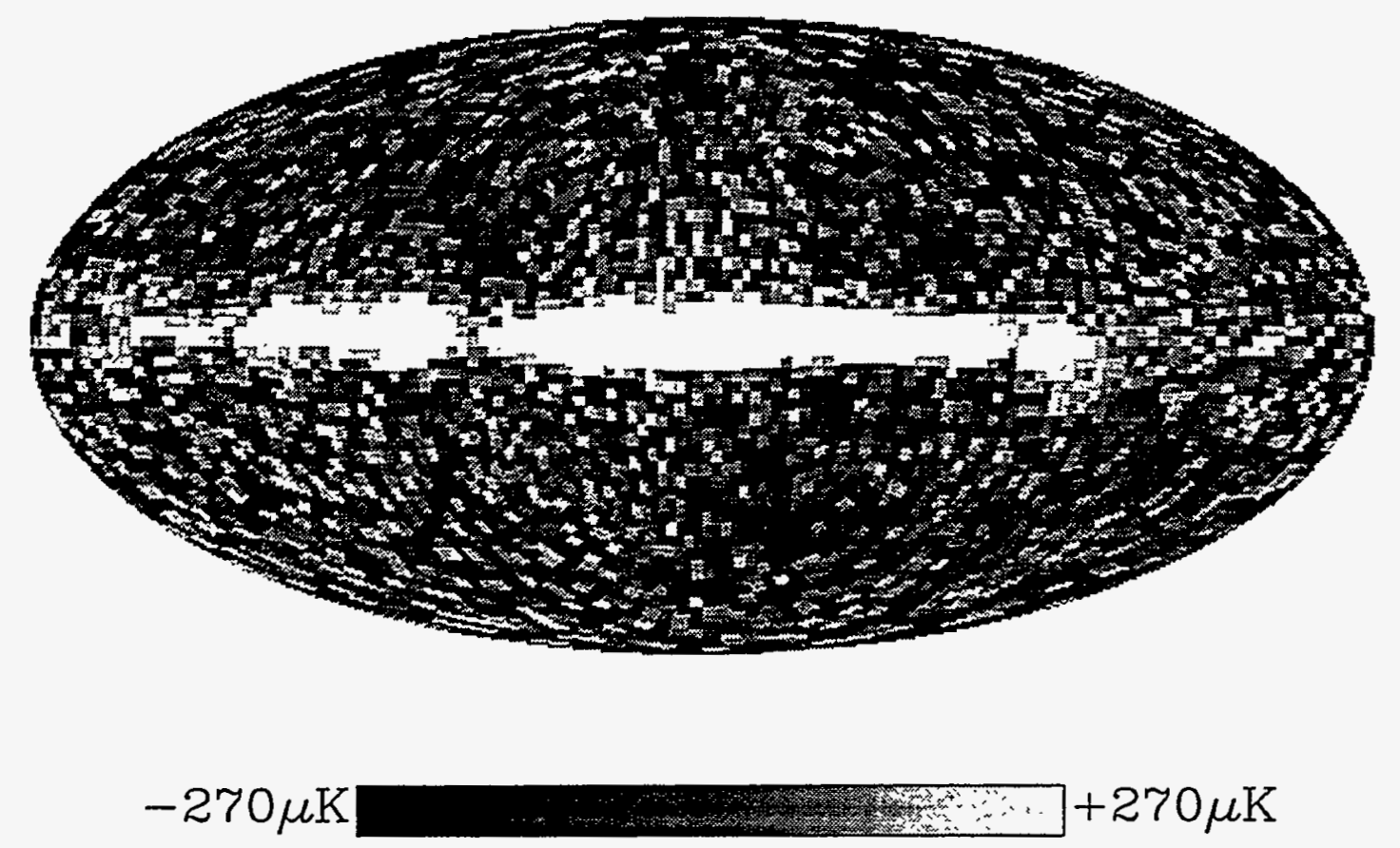

Figure 8.5: Yr2 Map. Weighted combination of $A+B$ 31, 53 and $90 \mathrm{GHz}$. Away from the Galactic plane several cool spots are recognizable in both maps. 


\subsection{Statistical Significance and Galactic Cuts}

The correlation function computed with different Galactic latitude cuts is an important tool used to establish that the signal observed is not of Galactic origin. Galactic cut independence of the Yr1 and Yr2 signal is indicated by the similarity of the curves for $\alpha \lesssim 20^{\circ}$ in Figures 8.6 and 8.7. There is consistency from year to year. These plots should be compared with the corresponding $2 \mathrm{Y}_{\mathrm{r}}$ curves in Figure 7.7.

We can quantify the Galactic latitude cut independence of the detected signal by computing $\chi^{2}$ values of the correlation function as a function of Galactic cut angle. For each of the correlation functions $\mathrm{Yr} 1, \mathrm{Yr} 2$ and $2 \mathrm{Yr}$ (Figures 8.6, 8.7 and 7.7 respectively), we compute

$$
\chi^{2}=\sum_{i=1}^{71}\left(\frac{C\left(\alpha_{i}\right)}{\sigma_{i}}\right)^{2},
$$

where 71 is the number of correlation function bins and the number of degrees of freedom (DOF) and $\sigma_{i}$ is the noise-only standard deviation of the $i$ th bin of the correlation function. The statistical significance of the detection above the noise in units of noiseonly standard deviations is then $\sqrt{\chi^{2}-D O F}$ and is listed in the last column of Table 8.1. The statistical significance of the signal detection in the $\mathrm{Yr} 1$ and $\mathrm{Yr} 2$ correlation functions is $>9 \sigma$ and $>10 \sigma$ respectively. The $Y_{r} 1$ statistical significance was reported as $>7 \sigma$ in Smoot et al. (1992) but the error bars on $C(\alpha)$ used to compute that value were more conservative than the noise-only error bars used here (see Figure 6.1).

Values for the rms temperature fluctuations in $\mathrm{Yr} 1$ and $\mathrm{Yr} 2$ maps can be derived in several ways from the correlation functions as described in Section 4.7. The results are listed in Table 7.3. The average of $20^{\circ}, 30^{\circ}$ and $40^{\circ}$ Galactic latitude cuts from the weighted crosscorrelation function of $53 \mathrm{GHz}$ with $90 \mathrm{GHz}$ yields $33 \pm 8 \mu \mathrm{K}$ and $40 \pm 7 \mu \mathrm{K}$ for $\mathrm{Yr} 1$ and $\mathrm{Yr} 2$ respectively. Analogous numbers for the case of the unweighted cross-correlation functions are $25 \pm 10 \mu \mathrm{K}$ and $43 \pm 6 \mu \mathrm{K}$ for $\mathrm{Yr}_{\mathrm{r}}$ and $\mathrm{Yr} 2$ respectively. Thus $\mathrm{Yr} 2$ seems to have a higher rms fluctuation level but one which could still be described as reasonably consistent with Yri.

One difference between the $Y_{I} 1$ and $Y_{I} 2$ data is the size of the quadrupole. The likelihood curves of the subtraction technique of Bennett et al. (1994) yield $Q_{r m s}=10 \pm 4$ for $\mathrm{Yr}_{r} 1, Q_{r m s} \sim 1 \pm 9$ for $\mathrm{Yr} 2$, and $Q_{r m s}=6 \pm 3$ for $2 Y_{r}$. The relatively small $\mathrm{Yr} 2 Q_{r m s}$ is evident in Figure 8.8 which compares the best-fit mean and dipole removed (md) correlation functions with the best-fit mean dipole and quadrupole removed (mdq) correlation functions. There is obviously a bigger difference between the md and mdq curve in Yr1 than there is in Yr2. The $Q_{r m s}$ values of Table 7.3 also reflect this tendency but less strongly: the weighted 
Table 8.1: Statistical Significance ${ }^{a}$ as a Function of Galactic Cut

\begin{tabular}{|cc|cc|ccc|}
\hline & & \multicolumn{2}{|c|}{$A-B^{6}$} & \multicolumn{3}{|c|}{$A+B^{b}$} \\
\hline Year & Galcut & $\chi^{2}$ & $\chi^{2} / D O F^{c}$ & $\chi^{2}$ & $\chi^{2} / D O F$ & Significance \\
\hline Yr1 & $10^{\circ}$ & 79 & 1.1 & 1040 & 14.6 & 31.1 \\
& $20^{\circ}$ & 76 & 1.1 & 235 & 3.3 & 12.8 \\
& $30^{\circ}$ & 64 & 0.91 & 168 & 2.4 & 9.9 \\
& $40^{\circ}$ & 66 & 0.93 & 152 & 2.2 & 9.0 \\
\hline Yr2 & $10^{\circ}$ & 67 & 0.94 & 1260 & 17.8 & 34.5 \\
& $20^{\circ}$ & 61 & 0.87 & 349 & 4.9 & 16.7 \\
& $30^{\circ}$ & 75 & 1.1 & 216 & 3.0 & 12.0 \\
& $40^{\circ}$ & 74 & 1.0 & 187 & 2.6 & 10.8 \\
\hline 2 Yr & $10^{\circ}$ & 60 & 0.84 & 4350 & 61.3 & 65.4 \\
& $20^{\circ}$ & 74 & 1.0 & 947 & 13.3 & 29.6 \\
& $30^{\circ}$ & 65 & 0.9 & 576 & 8.1 & 22.5 \\
& $40^{\circ}$ & 85 & 1.2 & 408 & 5.7 & 18.4 \\
\hline
\end{tabular}

aStatistical significance in units of noise-only standard deviations is $\sqrt{\chi^{2}-D O F}$, see equation (8.1).

${ }^{b}$ Cross-correlations of $53 \mathrm{GHz}$ with $90 \mathrm{GHz}$.

c The number of degrees of freedom DOF is 71 and is equal to the number of correlation function bins.

average of the results for Galactic latitude cuts of $20^{\circ}, 30^{\circ}$, and $40^{\circ}$ from the unweighted

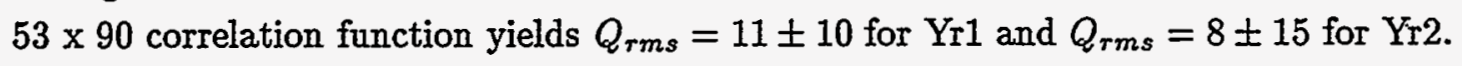




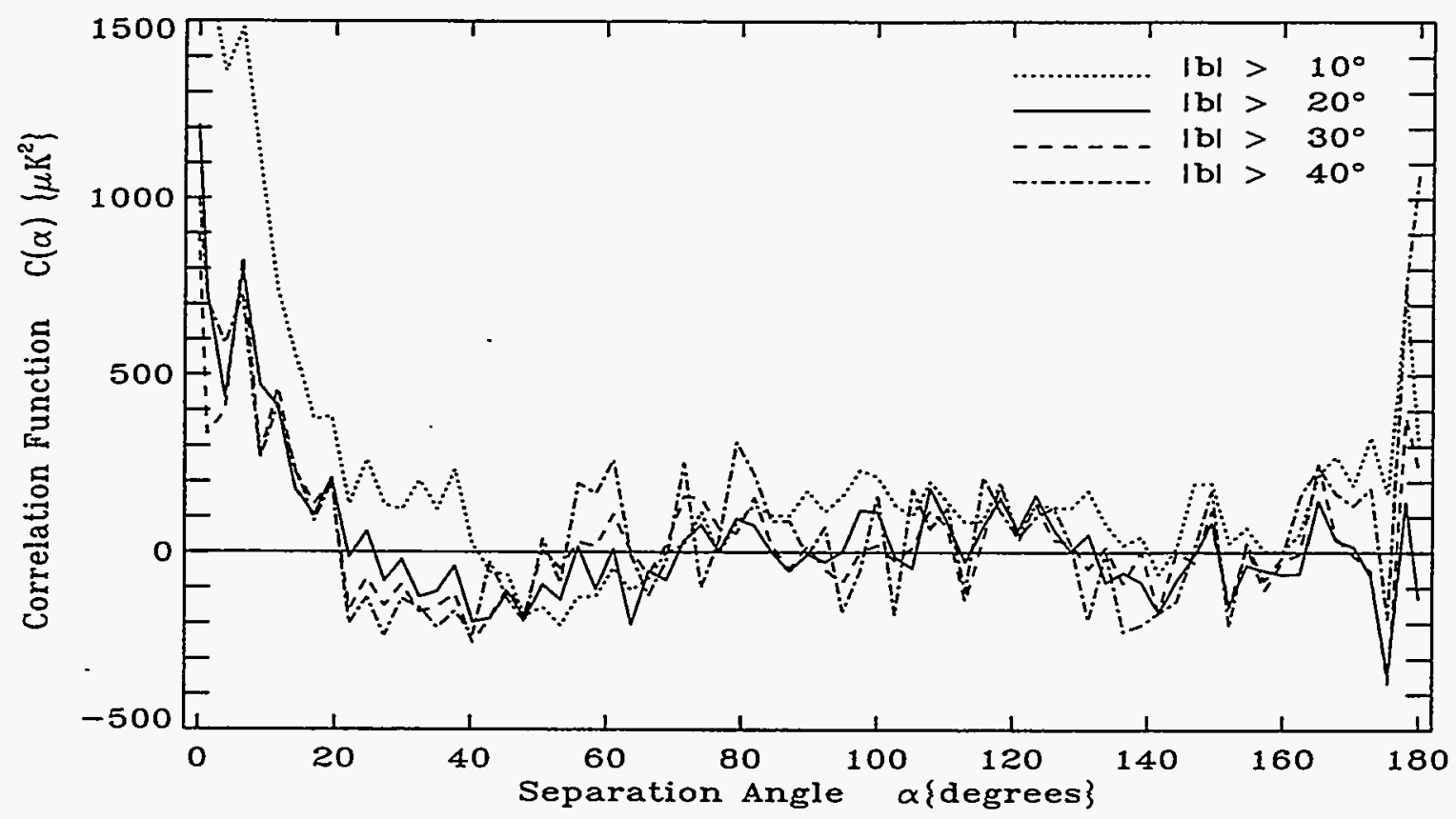

Figure 8.6: Yr1 Galactic Latitude Cut Independence. Cross-correlation functions of $\mathrm{Y}_{\mathrm{r} 1}$ A+B $53 \mathrm{GHz}$ with $90 \mathrm{GHz}$. Notice that the signal is very stable for $20^{\circ}, 30^{\circ}$ and $40^{\circ}$ cuts.

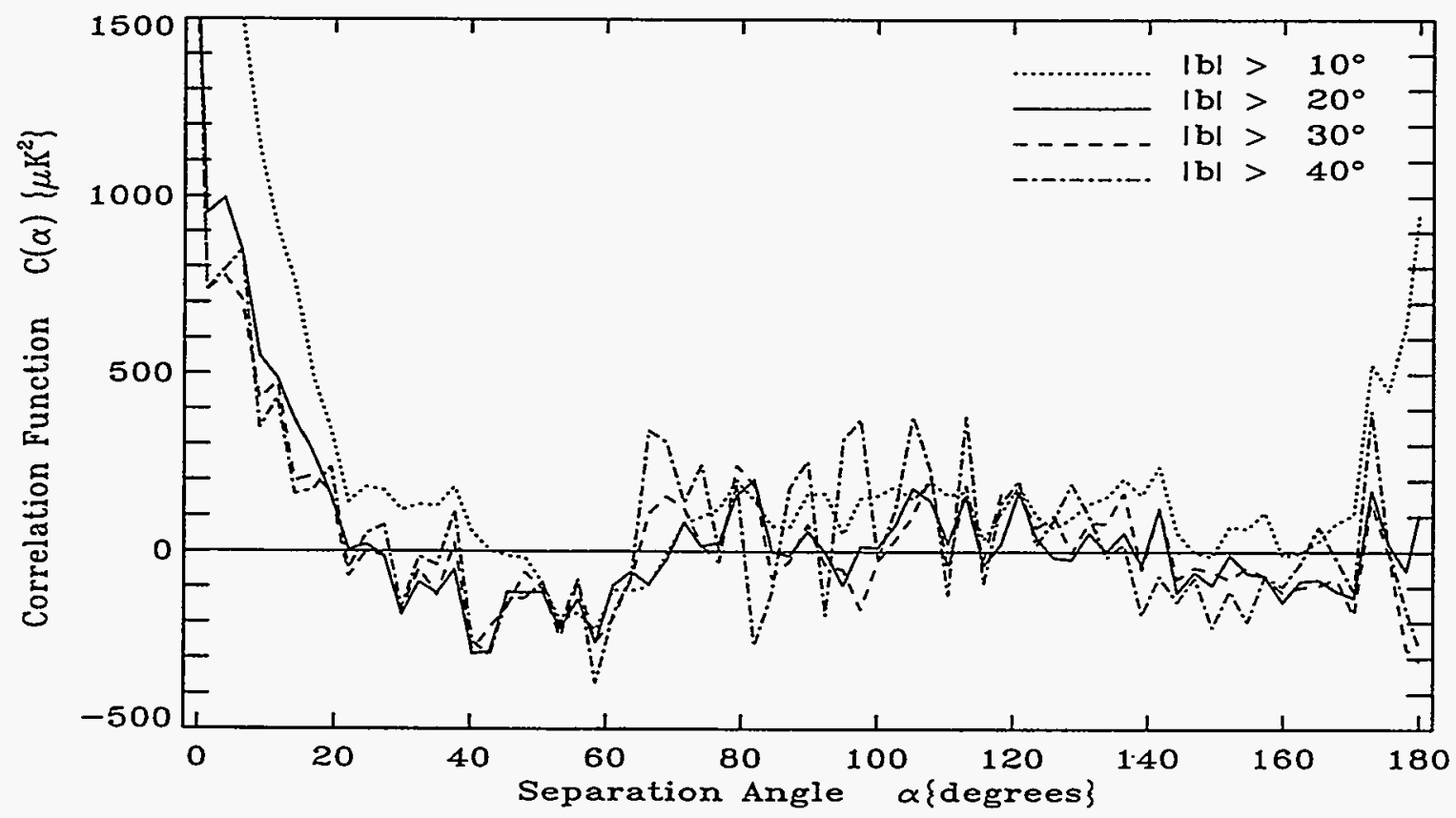

Figure 8.7: Yr2 Galactic Latitude Cut Independence. Same as previous figure except with Yr2 data. The signal is very stable for cuts of $20^{\circ}, 30^{\circ}$ and $40^{\circ}$. 

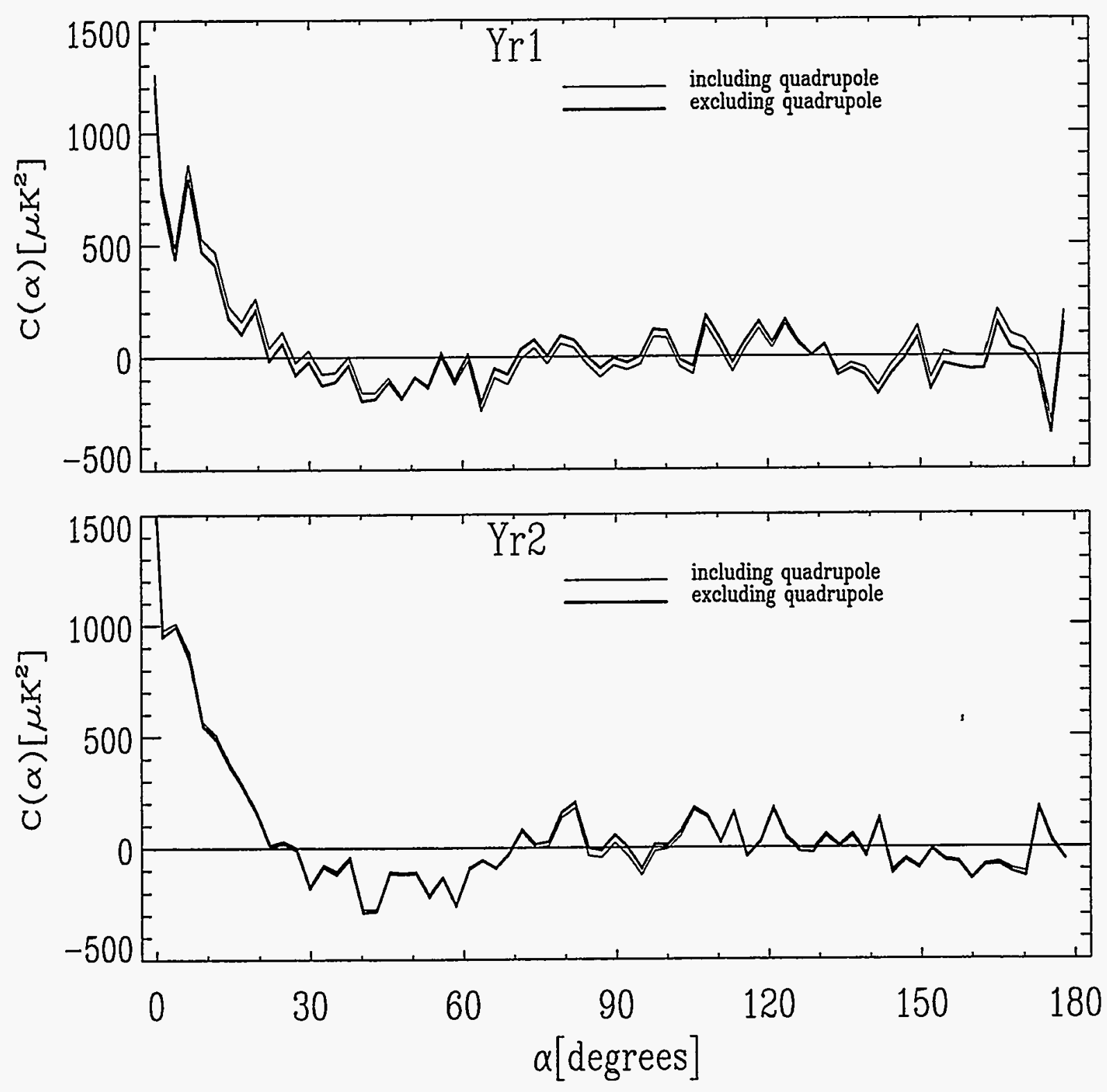

Figure 8.8: Yr1 and Yr2 Correlation Functions with and without quadrupole. These crosscorrelation functions of $53 \mathrm{GHz}$ with $90 \mathrm{GHz}$ are for $|b|>20^{\circ}$. Notice the relatively smaller quadrupole in Yr2 when compared with Yr1. 


\title{
Chapter 9
}

\section{Correlated Noise in the Maps}

\begin{abstract}
The COBE DMR sky maps contain low-level correlated noise. We obtain estimates of the amplitude and pattern of the correlated noise from three techniques: angular averages of the covariance matrix, Monte Carlo simulations of two-point correlation functions, and direct analysis of the DMR maps. The results from the three methods are mutually consistent. The noise covariance matrix of a DMR sky map is diagonal to an accuracy of better than $1 \%$. For a given sky pixel, the dominant noise covariance occurs with the ring of pixels at an angular separation of $60^{\circ}$ due to the $60^{\circ}$ separation of the DMR horns. The mean covariance at $60^{\circ}$ is $0.45 \%_{-0.14}^{+0.18}$ of the mean variance. Additionally, the variance in a given pixel is $0.7 \%$ greater than would be expected from a single beam experiment with the same noise properties. Auto-correlation functions suffer from $a \sim 1.5 \sigma$ positive bias at $60^{\circ}$ while cross-correlations have no bias. Published COBE DMR results are not significantly affected by correlated noise.
\end{abstract}

\subsection{Introduction}

The $C O B E$ collaboration has reported the detection of anisotropies in the cosmic microwave background radiation (Smoot et al. 1992, Bennett et al. 1994). These anisotropies cannot be attributed to known systematic effects (Kogut et al. 1992) or any known Galactic or extragalactic foreground (Bennett et al. 1992b, 1993). Concern about the magnitude of correlated noise in the sky maps has led to this investigation. 
The DMR measures temperature differences across the sky using pairs of horn antennas with a fixed $60^{\circ}$ separation angle (Smoot et al.1990). The construction of a DMR temperature map from measurements of temperature differences involves a least-squares fit during which essentially uncorrelated measurement errors become correlated temperature errors in the sky map. The structure of this correlated noise is fully described by the pixel temperature covariance matrix which depends only on the $60^{\circ}$ horn separation angle and the details of the sky coverage. In this Chapter we quantify the amplitude and angular dependence of the correlated noise. To first order, the noise in the DMR maps is uncorrelated. The dominant correlation in each pixel is due to the average noise in a ring $60^{\circ}$ away. The mean covariance at $60^{\circ}$ is $0.45 \%$ of the mean variance. This level of correlation will slightly bias auto-correlation functions and their derivatives but previously published DMR results are not significantly affected by correlated noise. The dominant correlated noise term in the DMR maps was discussed briefly in Wright et al. (1994a) and Wright (1994).

In this chapter we examine the noise in the DMR maps. In Section 9.2 we describe the covariance matrix and its inverse. In Section 9.3 we use three methods to obtain estimates of the correlated noise in DMR maps and in Section 9.4 we discuss the effect of the autocorrelation bias on determination of the cosmological parameters $n, Q_{r m s-P S}$ and the rms temperature fluctuation amplitude.

\subsection{Pixel Covariance Matrix $\mathbf{A}^{-1}$}

In Section 3.2 we described how maps were made from the differential data and we sketched the role of the matrix $A$ in producing a map. The noise in the maps is explicit in equation 3.8:

$$
\vec{T}_{\text {obs }}=\vec{T}+\vec{n}=\vec{T}+\mathbf{A}^{-1} \vec{\delta}
$$

In this Section we describe $\mathbf{A}$ and discuss how its properties determine $\mathbf{A}^{-1} \cdot \mathbf{A}$ is known as the cross-products matrix, the moment matrix and/or the curvature matrix since $A_{i j}=-\frac{1}{2} \frac{\partial \chi^{2}}{\partial T_{i} \partial T_{j}}$. A is a 6144 by 6144 symmetric, sparse and singular matrix. We make $\mathrm{A}$ non-singular (invertible) by augmenting the diagonal terms by some small number $\epsilon$. This does nothing more than impose an arbitrary mean level on an otherwise unique solution (see Appendix J). 
The 6144 diagonal elements of $\mathrm{A}$ are the number of observations $\left(N_{i}\right)$ of the 6144 pixels.

$$
\mathbf{A}=\left(\begin{array}{cccccccc}
N_{1} & 0 & -N_{1,3} & 0 & 0 & 0 & 0 & \ldots \\
0 & N_{2} & 0 & 0 & 0 & 0 & 0 & \ldots \\
-N_{1,3} & 0 & N_{3} & 0 & 0 & 0 & 0 & \ldots \\
0 & 0 & 0 & N_{4} & 0 & 0 & -N_{4,7} & \ldots \\
0 & 0 & 0 & 0 & N_{5} & 0 & 0 & \ldots \\
0 & 0 & 0 & 0 & 0 & N_{6} & 0 & \ldots \\
0 & 0 & 0 & -N_{4,7} & 0 & 0 & N_{7} & \ldots \\
\vdots & \vdots & \vdots & \vdots & \vdots & \vdots & \vdots & \ddots
\end{array}\right)
$$

A column of $\mathrm{A}$ is plotted as a map in Figure 9.1. For one year, the average value of $N_{i}$ is $\bar{N} \approx 17,500$. $N_{i j}$ is the number of times pixel $i$ and $j$ have been differenced. When $\alpha_{i j} \approx 60^{\circ} A_{i j}=A_{j i}=-N_{i j}$, otherwise $A_{i j}=0$. The average number of coupled pixels, i.e., the average number of non-zero off-diagonal elements in a row or column of an $\mathbf{A}$ from one year of observations, is $N_{\text {ref }}=273$. This is the number of pixels in the "reference ring" at $60^{\circ} \pm 3^{\circ}$ separation from the diagonal pixel. The average value of these 273 off-diagonal $N_{i j}$ elements is $\overline{N_{i j}}=\frac{\bar{N}}{N_{\text {ref }}}=64$. Only $4 \%\left(=\left(N_{\text {ref }}+1\right) / 6144\right)$ of $\mathrm{A}$ is non-zero. A has very little channel dependence. Since $\mathbf{A}$ is symmetric, $\mathbf{A}^{-1}$ is symmetric. Since $\mathbf{A}$ is diagonally dominant we expect $\mathrm{A}^{-1}$ to resemble the form below (as $N_{i j} \rightarrow 0, \mathrm{~A}^{-1} \rightarrow$ diagonal).

$$
\mathrm{A}^{-1} \sim\left(\begin{array}{cccccccc}
\frac{1}{N_{1}} & 0 & \frac{N_{1,3}}{N_{1} N_{3}} & 0 & 0 & 0 & 0 & \ldots \\
0 & \frac{1}{N_{2}} & 0 & 0 & 0 & 0 & 0 & \ldots \\
\frac{N_{1,3}}{N_{1} N_{3}} & 0 & \frac{1}{N_{3}} & 0 & 0 & 0 & 0 & \ldots \\
0 & 0 & 0 & \frac{1}{N_{4}} & 0 & 0 & \frac{N_{4,7}}{N_{4} N_{7}} & \ldots \\
0 & 0 & 0 & 0 & \frac{1}{N_{5}} & 0 & 0 & \ldots \\
0 & 0 & 0 & 0 & 0 & \frac{1}{N_{6}} & 0 & \ldots \\
0 & 0 & 0 & \frac{N_{4,7}}{N_{4} N_{7}} & 0 & 0 & \frac{1}{N_{7}} & \ldots \\
\vdots & \vdots & \vdots & \vdots & \vdots & \vdots & \vdots & \ddots
\end{array}\right)
$$

A column of $\mathrm{A}^{-1}$ is plotted as a map in Figure 9.2. $\mathrm{A}^{-1}$ is the covariance matrix of the fitted temperatures. The diagonal elements are the variances of the fitted temperatures: $<\delta \hat{T}_{i} \delta \hat{T}_{j}>=A_{i j}^{-1}$. Notice that the dominant off-diagonal terms at $60^{\circ}$ from the diagonal are all positive. Therefore we expect a positive correlation between pixels separated by $60^{\circ}$. $\mathrm{A}$ column of $\mathrm{A}$ and $\mathrm{A}^{-1}$ are displayed as skymaps in Figures 9.1 and 9.2. 

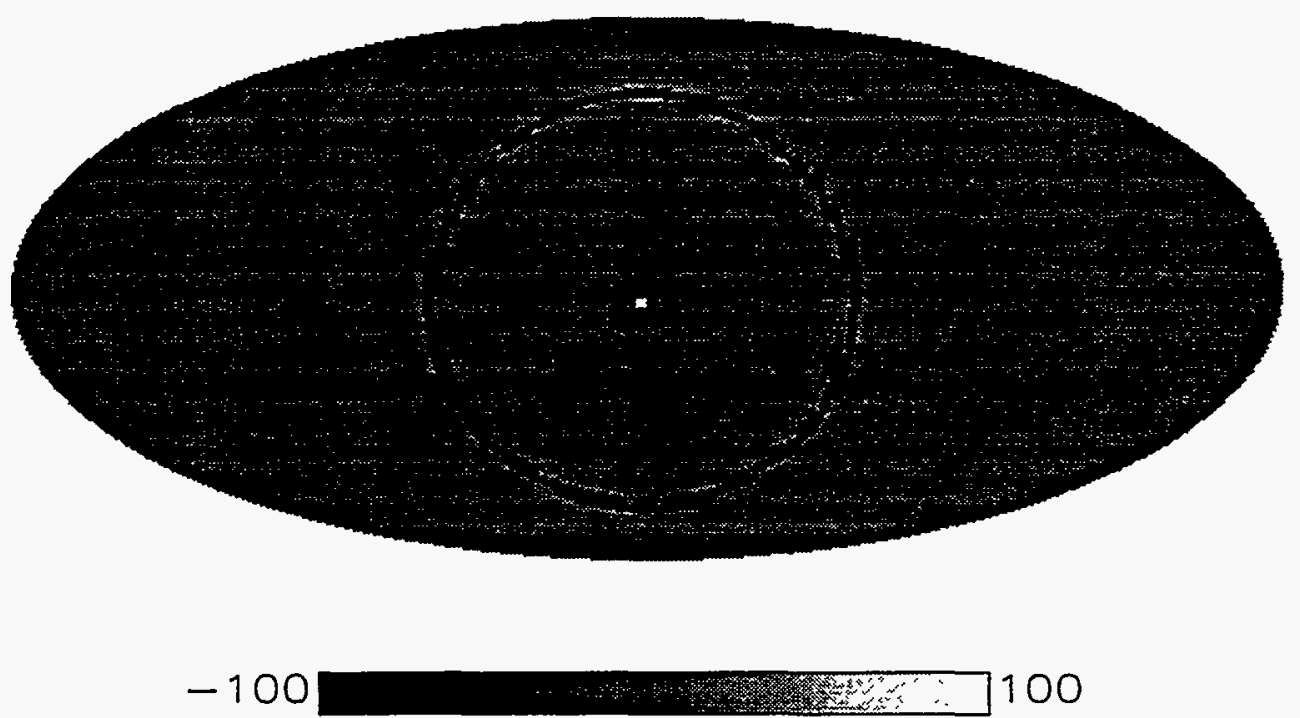

Figure 9.1: Column 1448 of the matrix A from 90A first year data. The central pixel (pixel 1448 ) and its reference ring, at $\approx 60^{\circ}$ angular separation, are the only non-zero elements.

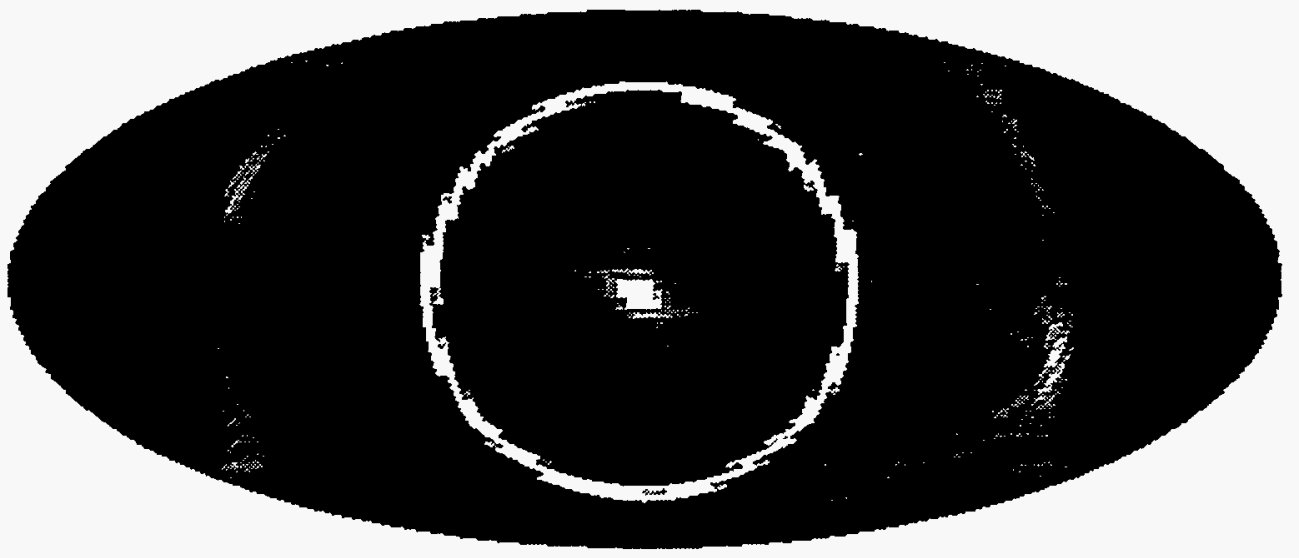

$-0.0005$ 0.0010

Figure 9.2: Column number 1448 of the matrix $\mathrm{A}^{-1}(\times N)$ (same column as above). The greyscale has been chosen to show the structure in the off-diagonal terms at the expense of saturating the central pixel (value $=1.005$ ) and the reference ring (values $\approx 0.003$ ). 
The approximate radial symmetry of both maps about the diagonal pixel located at the center of the map is apparent. The central pixel has been observed 11,933 times $\left(N_{1448}=11,933\right)$.

\subsection{Correlated Noise Estimates}

\subsubsection{Correlated Noise Estimates from the Covariance Matrix}

The covariance matrix of the sky map temperatures fully describes the noise properties of the map. The diagonal elements are the variances of the fitted temperatures while the non-zero off-diagonal elements represent correlated noise in the maps. We use equation (9.1) and the uncorrelated nature of the measurement errors to obtain the covariance matrix of the sky map temperatures,

$$
\operatorname{Cov}\left(\vec{T}_{o b s}\right) \equiv<\vec{n} \vec{n}^{T}>=\left[\mathbf{A}^{-1} \mathbf{V}^{T}\right] \operatorname{Cov}(\vec{\Delta})\left[\mathbf{A}^{-1} \mathbf{V}^{T}\right]^{T}=\sigma_{c h}^{2} \mathbf{A}^{-1}
$$

Thus, the diagonal elements of $\sigma_{c h}^{2} \mathrm{~A}^{-1}$ are the variances of the fitted temperatures. For 350 randomly chosen values of $i$ we find that $\sigma_{c h}^{2} A_{i i}^{-1}=\left(\sigma_{c h}^{2} / N_{i}\right)(1.007 \pm 0.003)$, where $N_{i}$ is the number of times pixel $i$ was observed. Thus, the solution of the normal equations produces a $0.7 \%$ increase over the variance expected from a single-beam experiment $\sigma_{c h}^{2} / N_{i}$.

The matrix $A^{-1}$ is symmetric and non-sparse. Since a column of $A^{-1}$ has 6144 elements, it can be conveniently viewed as a pixelized map of the correlations of each pixel with the central pixel (i.e. with the diagonal element). Figure 9.2 shows that the pattern of the correlations is approximately radially symmetric around the central pixel. For each of the 350 randomly chosen columns, we calculate normalized averages of annular regions of the off-diagonal elements

$$
A_{i}^{-1}(\alpha)=\frac{1}{W} \frac{\sum_{k}^{\alpha_{i k}=\alpha} A_{i k}^{-1}}{A_{i i}^{-1}},
$$

where all pixels in the annuli have constant angular separation from the central pixel and $W=\sum_{k}^{\alpha_{i k}=\alpha} 1$. The $2.6^{\circ}$ pixel size is used as a binning width. Figure 9.3 shows the average $<A^{-1}(\alpha)>$ and $68 \%$ confidence levels of the 350 randomly chosen columns. The mean covariance at $60^{\circ}$ is $0.45 \%_{-0.14}^{+0.18}$ of the mean variance. Thus the noise correlations in the DMR maps are small and the noise properties resemble those of a single-beam experiment. The value of the zero lag bin is off scale; $\left\langle A^{-1}(0)\right\rangle=1.007$, and is the source of the $0.7 \%$ excess variance reported above. This analysis is based on the normal equations from the 


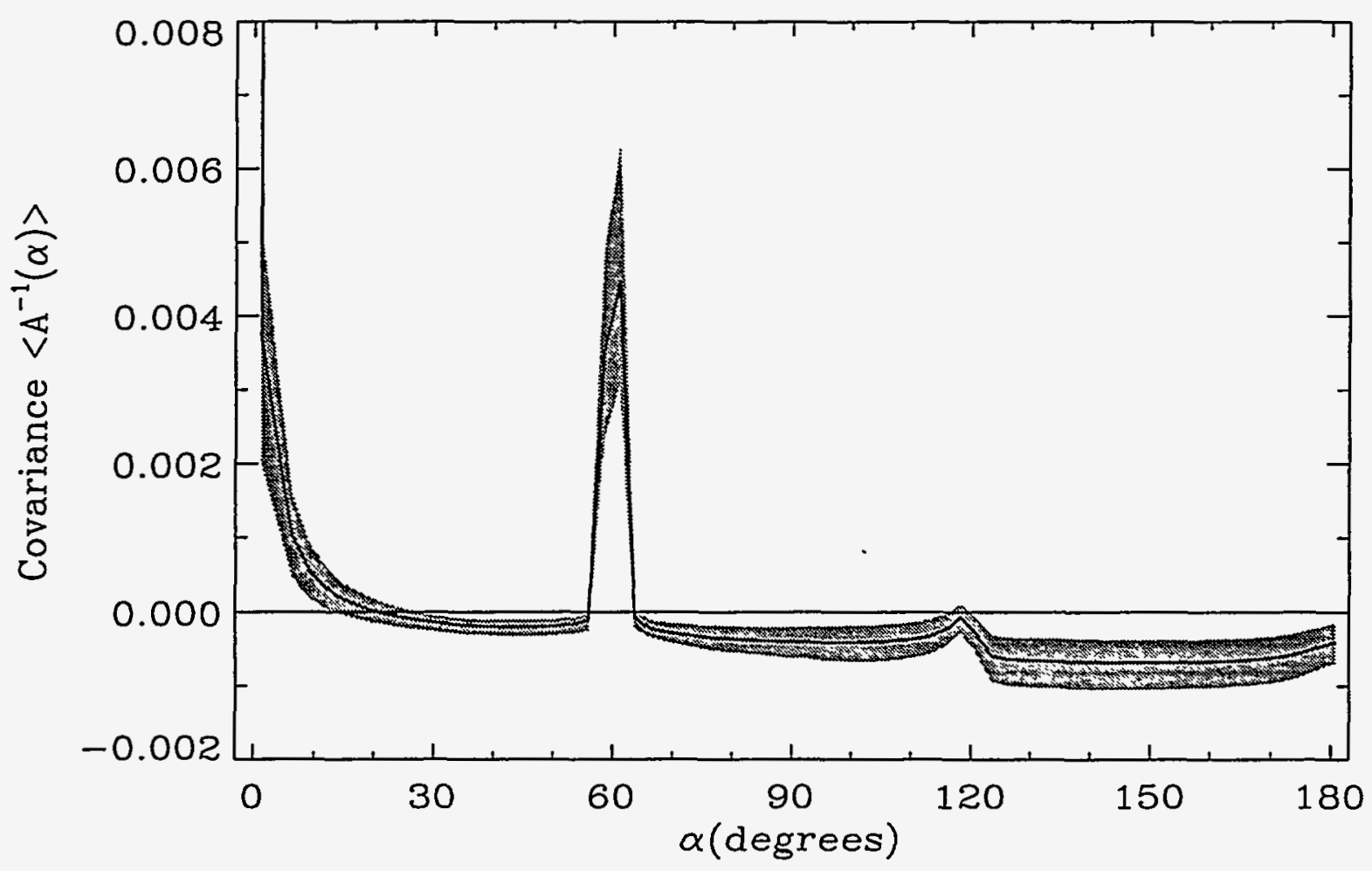

Figure 9.3: The average radial structure of the pixel temperature covariance matrix $\mathbf{A}^{-1}$ (see equation 9.5 ). The shaded band marks the $68 \%$ confidence levels.

first year data of channel 53A. Since the sky coverage and the $60^{\circ}$ horn separation are only weakly channel dependent, the covariance matrices of the other channels are essentially the same and time independent.

\subsubsection{Correlated Noise Estimates from Monte Carlo Simulations}

The two-point auto-correlation function of a pixelized DMR map is the weighted average product of temperatures separated by angle $\alpha$

$$
C(\alpha)=\frac{1}{W} \sum_{i, j} w_{i} w_{j} T_{o b s, i} T_{o b s, j},
$$

where the sum is over all pixel pairs $i j$ whose separation angle $\alpha_{i j}$ lies within half a bin width of $\alpha$. We use the weights $w_{i}=1 / \operatorname{var}\left(T_{o b s, i}\right)$ and approximate $\operatorname{var}\left(T_{o b s, i}\right)$ by $\sigma_{c h}^{2} / N_{i}$. The normalization factor is $W=\sum_{i, j} w_{i} w_{j}$. For cross-correlation functions $i$ refers to one 

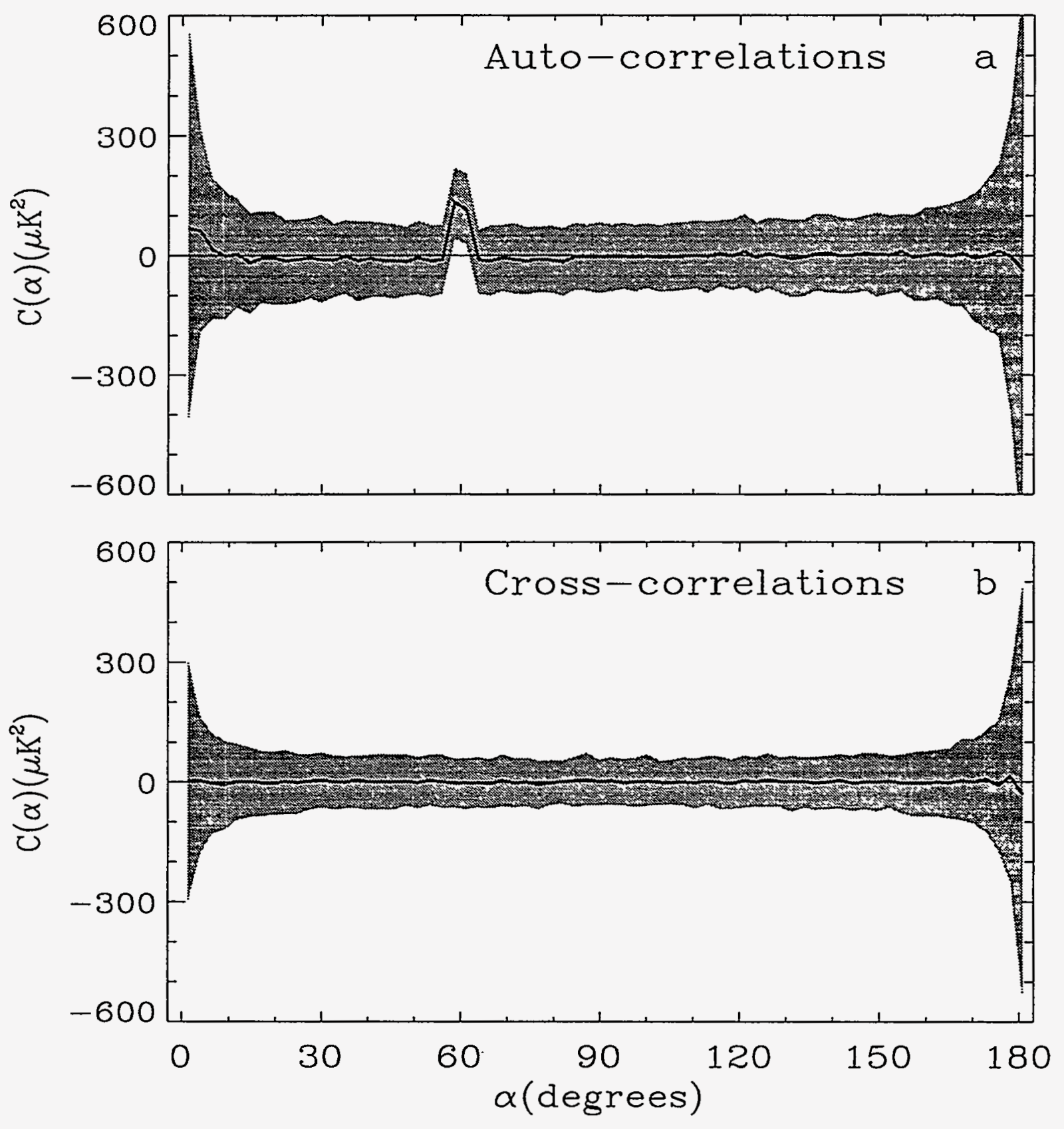

Figure 9.4: a) The 2-point auto-correlation of 1000 simulated 53A single-year noise maps. A $20^{\circ}$ Galactic cut has been made. The best-fit mean, dipole and quadrupole have been removed from each simulation. Weighted $\mathrm{C}(\alpha)$ 's were used. The shaded band marks the $68 \%$ confidence levels. b) Cross-correlations from the same simulated noise maps as (a). No bias is evident. 
map and $j$ to the other. Using equation (9.1) the auto-correlation function is defined as

$$
C(\alpha)=\frac{1}{W} \sum_{i j}^{\alpha_{i j}=\alpha} w_{i} w_{j}\left(T_{i}+n_{i}\right)\left(T_{j}+n_{j}\right) .
$$

The signal-noise cross-terms in equation (9.7) contribute to the uncertainty in $C(\alpha)$ but do not contain a bias. Auto-correlation functions of DMR maps are biased only by the noise-noise cross-term. To examine these biases we insert only instrument noise into the measurement vector $\vec{M}$ of the normal equations. We then compute the ensemble average of the correlation functions of the resulting noise maps and obtain,

$$
\begin{aligned}
\langle C(\alpha)\rangle & =\frac{1}{W} \sum_{i j}^{\alpha_{i j}=\alpha} w_{i} w_{j}\left\langle n_{i} n_{j}>\right. \\
& =\frac{1}{W} \sum_{i j}^{\alpha_{i j}=\alpha} w_{i} w_{j} \sigma_{c h}^{2} A_{i j}^{-1} .
\end{aligned}
$$

Figure 9.4 shows the bias in the auto-correlation function from 1000 simulations of single- year 53A noise maps. One can see a positive bias of $\sim 1.5 \sigma$ at $60^{\circ}$, where $\sigma$ is the half-width of the gray band, and a smaller positive bias close to $0^{\circ}$. The bias does not change substantially when a signal is included in the simulations. Figure $2 b$ shows that cross-correlations do not suffer from the pixel-pixel noise correlations.

The positive correlation between noise in pixels separated by $60^{\circ}$ can be explained as follows: the total noise in each pixel includes contributions from the off-diagonal elements of the covariance matrix $\sum_{j} A_{i j}^{-1} \delta_{j}, j \neq i$. These contributions are dominated by the average temperature of the noise in the reference ring at $60^{\circ}$ angular separation. Thus the central pixel is positively correlated with the reference ring.

The bias at $60^{\circ}$ in Figure 9.4 can be approximately related to the the mean covariance at $60^{\circ}$ obtained in the previous Section. Although the two methods differ in weighting and normalization we find $C\left(60^{\circ}\right) / C\left(0^{\circ}\right)=0.41 \% \pm 0.22$. The size of the biases in Figure 9.4 scale with the variance of the noise in the maps. For example, the sizes of the biases in the auto-correlations of two year maps are half the sizes of the biases in the one year maps. The bias at $60^{\circ}$ is not obvious in any single realization of the 1000 used to make Figure 9.4. Similarly the bias is not apparent in the auto-correlations of Figure 2 of Smoot et al. (1992) or Figure 2 of Wright et al. (1992). 


\subsubsection{Correlated Noise Estimates from DMR Maps}

An estimate of the bias can be obtained using only the data. We take advantage of the previous result that the auto-correlations contain the bias while the cross-correlations do not. Their difference is then an estimate of the bias. We introduce the quantity $B(\alpha)$ as an empirical estimate of the bias defined as

$$
B(\alpha)=\frac{1}{12} \sum_{m=1}^{6}\left[\frac{C_{A, m}(\alpha)-C_{A \times B, m}(\alpha)}{\sigma_{A, m}(\alpha)}+\frac{C_{B, m}(\alpha)-C_{A \times B, m}(\alpha)}{\sigma_{B, m}(\alpha)}\right],
$$

where $C_{A, m}, C_{B, m}$ and $C_{A \times B, m}$ are respectively, the auto-correlation of channel A, autocorrelation of channel $\mathrm{B}$ and their cross-correlation, for six values of $m$ (2 years $\times 3$ frequencies). These correlation functions are computed using equation (9.6). The empirical bias $B(\alpha)$ is expressed in dimensionless units of noise-only standard deviations of the autocorrelations and is plotted in Figure 9.5. The bias at $60^{\circ}$ is evident.

\subsection{Cosmological Implications}

The bias in Figure 9.4 near $\alpha=0^{\circ}$ increases the amplitude of rms temperature fluctuations determined from the first non-zero bin of auto-correlations. The $C(0)^{1 / 2}=$ $36 \pm 5 \mu \mathrm{K}$ quoted in Bennett et al.(1994) is from the zeroth bin of the $53 \times 90 \mathrm{GHz}$ cross-correlation and is not susceptible to noise correlation bias. The correlated noise near $\alpha=0^{\circ}$ does not correlate across channels and therefore contributes the same to both the $(\mathrm{A}+\mathrm{B}) / 2$ and $(\mathrm{A}-\mathrm{B}) / 2$ maps. Therefore $\sigma_{s k y}=\sqrt{\sigma_{A+B}^{2}-\sigma_{A-B}^{2}}$ is unbiased and the previously published values of rms temperature fluctuations (Smoot et al. 1992, Bennett et al. 1994) are not affected by correlated noise.

The noise correlations at $60^{\circ}$ put additional small angular scale $(\ell \gtrsim 6)$ power into the maps and can bias determinations of the power spectral index $n$ and amplitude $Q_{r m s-P S}$ from auto-correlations. We estimate the magnitude of this effect by adding many realizations of correlated noise to a simulated sky, and comparing the values for $n$ and $Q_{r m s-P S}$ from $\chi^{2}$ minimization of the auto- and cross correlation functions, analogous to the treatment in equation (9.10). For noise equivalent to the $53 \mathrm{~A}$ one-year map the fitted values are $n_{\text {bias }}=0.04 \pm 0.04$ and $Q_{\text {bias }}=1.6 \pm 0.4$. The bias on $n$ is negligible and the small bias on $Q_{r m s-P S}$ is probably due to the slight increase in the autocorrelation function seen in Figure 9.4 at angles smaller than $10^{\circ}$. The biases are even smaller when analyzing 


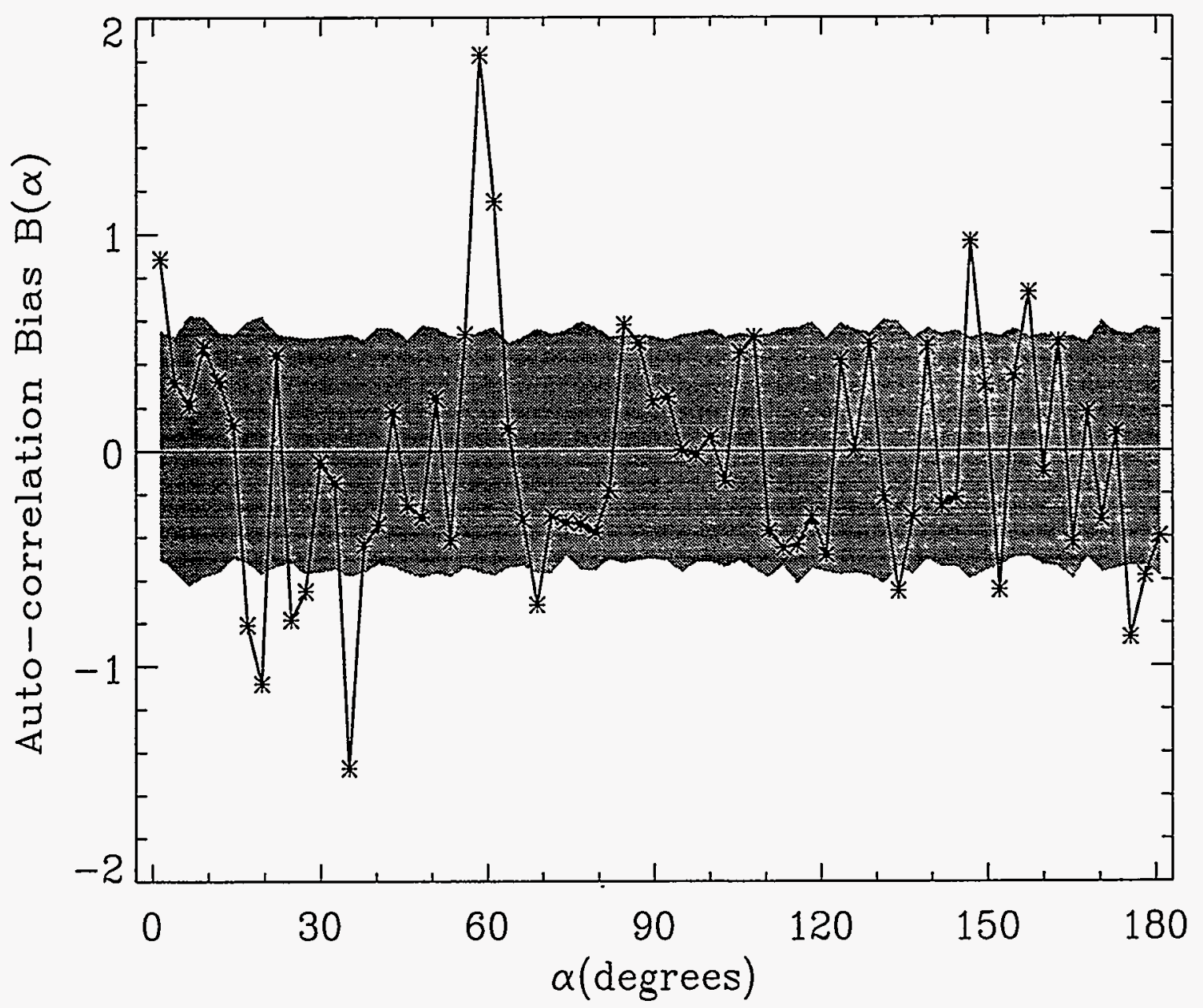

Figure 9.5: The auto-correlation function bias with respect to the corresponding crosscorrelations. $\mathrm{B}(\alpha)$ is computed from twelve independent DMR data sets (2 years $\times 3$ frequencies $\times 2$ channels). See equation (9.10) of text for the definition of $B(\alpha)$. The shaded band marks the $95 \%$ confidence levels from 250 simulations with uncorrelated noise. The best-fit mean, dipole and quadrupole have been removed before $B(\alpha)$ was calculated. The result is not strongly dependent on which multipoles are removed. Weighted $C(\alpha)$ 's were used. 
multi-channel and multi-year DMR data. Thus, the published COBE DMR results are not significantly affected by correlated noise.

\subsection{Summary}

We have investigated the structure and magnitude of the correlated noise in DMR maps using the covariance matrix of the pixel temperatures, two-point correlation functions of Monte Carlo simulations and the output maps themselves. The noise in the DMR maps resembles that from a single-beam experiment in that the pixel-pixel noise correlations are small. The approximately radial structure of the pixel noise covariance matrix is shown in 9.3. The dominant correlation in each pixel is from the average noise in a ring $60^{\circ}$ away. The mean covariance at $60^{\circ}$ is $0.45 \%$ of the mean variance. The noise variance is $0.7 \%$ larger than the $\sigma_{c h}^{2} / N_{i}$ value expected from a single-beam experiment. At $\alpha=60^{\circ}$, auto-correlations suffer from a bias whose magnitude is 1.5 times the uncorrelated noise uncertainty. Cross-correlation functions are not affected by noise correlations and should be used for $n, Q_{r m s-P S}$ and $C(0)^{1 / 2}$ determinations, thus previously published DMR results are not significantly affected by correlated noise. 


\title{
Chapter 10
}

\section{Estimates of $n$ and $Q_{r m s-P S}$ from the Correlation Function}

\begin{abstract}
We obtain the power law spectral index $n$ and the quadrupole normalization $Q_{r m s-P S}$ from the correlation functions of $\mathrm{Yr} 1$, Yr2 and 2Yr DMR maps. A $\chi^{2}$ fit to the 2Yr correlation function excluding the quadrupole yields $n=1.2_{-1.0}^{+0.6}$ and $Q_{r m s-P S}=17.3_{-5.1}^{+7.1}$. Including the quadrupole raises $n$ by $\approx 0.5$ and lowers $Q_{r m s-P S}$ by $\approx 4 \mu \mathrm{K}$. Excluding the quadrupole, the best fit $n=1$ normalization is $Q_{r m s-P S}=18.6_{-2.4}^{+3.2}$. We compare our results to other published $n$ and $Q_{r m s-P S}$ determinations.
\end{abstract}

\subsection{The $n$ and $Q_{r m s-P S}$ Parametrization of the Power Spectrum}

The large angular scale solution to the collisionless Boltzmann equation gives a power spectrum parametrized by two numbers: the shape $n$ and the amplitude $Q_{r m s-P S}^{2}$ (see Section 4.3)

$$
<\Delta T_{\ell}^{2}\left(n, Q_{r m s-P S}\right)>=Q_{r m s-P S}^{2} \frac{(2 \ell+1)}{5} \frac{\Gamma\left(\ell+\frac{n-1}{2}\right) \Gamma\left(\frac{9-n}{2}\right)}{\Gamma\left(\ell+\frac{5-n}{2}\right) \Gamma\left(\frac{3+n}{2}\right)} .
$$

Equation (10.1) is often used to normalize models and data. The DMR is sensitive enough to high $\ell$ values to be effected by Fourier components which were excluded from the integral (equation (4.8)) used to derive equation (10.1). For a broad class of reasonable models, the 
wings of the Doppler peak extend into the $\ell$ space sampled by the DMR. This produces a tilt in the apparent power spectral index relative to what one would measure if one were sensitive to only the first few $\ell$ components. Wright et al. (1994b) refer to a "primordial" $n_{p r i}$ and an "apparent" $n_{a p p}$. For the DMR, the standard CDM tilt or bias consists of $\Delta n=0.15$ (Bond 1994). In other words, if the mass-overdensity power spectrum were a perfect $n_{p r i}=1(P(k) \propto k)$, the DMR would measure $n_{\text {app }}=1.15$. In this Chapter, we fit the DMR correlation function to the $\Delta T_{\ell}^{2}$ of equation (10.1) and we find an apparent $n$. We are fitting a model function that is appropriate in low $\ell$ space to an observed power spectrum which is presumably slightly affected by model dependent effects at higher $\ell$. We do not correct for this model dependent bias.

\section{2 $\chi^{2}$ fitting for $n$ and $Q_{r m s-P S}$}

\subsubsection{Method}

A model correlation function can be expressed in terms of the $n$ and $Q_{r m s-P S}^{2}$ dependent power spectrum of equation (10.1)

$$
C_{n}(\alpha)=\sum_{\ell=2}^{\ell_{\max }}\left\langle\Delta T_{\ell}^{2}\right\rangle W_{\ell}^{2} P_{\ell}(\cos \alpha)
$$

where $W_{\ell}$ are Legendre expansion coefficients of the DMR beam. We want to find the $n$ and $Q_{\tau m s-P S}^{2}$ values which produce the correlation function $C_{n}(\alpha)$ that most closely fits the measured $C_{d}(\alpha)$. One simple method is a $\chi^{2}$ fit to the data

$$
\chi_{n}^{2}=\sum_{i}^{i_{\max }} \frac{\left(C_{d}(i)-C_{n}(i)\right)^{2}}{\sigma_{d}^{2}(i)+\sigma_{n}^{2}(i)}
$$

where $C_{d}(i)$ and $\sigma_{d}(i)$ are the correlation function and noise-only error bar of the $i$ th bin of the data, $C_{n}$ is from equation (10.2) and $\sigma_{n}^{2}(i)$ is the cosmic variance (Section 4.4). Since $C_{n}\left(\alpha_{i}\right)$ is linear in $Q_{r m s-P S}^{2}$ we set

$$
\frac{\partial \chi_{n}^{2}}{\partial Q_{r m s-P S}^{2}}=0
$$

and solve for $Q_{\tau m s-P S}^{2}$. Plugging this $Q_{\tau m s-P S}^{2}$ back into equation (10.3) we obtain a value for $\chi_{n}^{2}$. We do this for a range of $n$ values and at the end choose the lowest $\chi_{n}^{2}$. That gives 
us a best fit $Q_{r m s-P S}^{2}$ and a best fit $n$. Schematically

$$
\left\{\begin{array}{ccc}
n_{1} & \rightarrow & \chi_{n_{1}}^{2} \\
n_{2} & \rightarrow & \chi_{n_{2}}^{2} \\
n_{3} & \rightarrow & \chi_{n_{3}}^{2} \\
& \vdots
\end{array}\right\} \text { choose lowest } \equiv \chi_{\min }^{2} \longrightarrow \text { best fit } n, Q_{r m s-P S}
$$

\subsubsection{Results}

Figure 10.1 shows the contours for the best $\chi^{2}$ fit power spectrum parameters $n$ and $Q_{r m s-P S}$ to the $2 \mathrm{yr} 53 \mathrm{~A}+\mathrm{B} \times 90 \mathrm{~A}+\mathrm{B}$ cross-correlation with mean, dipole and quadrupole excluded. The minimum $\chi^{2}$ is at $n=1.2_{-1.0}^{+0.6}$ and $Q_{r m s-P S}=17.3_{-5.1}^{+7.1}$. The best fit $n=1$ normalization for this case is $Q_{r m s-P S}=18.6_{-2.4}^{+3.2}$. When the quadrupole is included the result is $n=1.7_{-0.9}^{+0.5}$ and $Q_{r m s-P S}=13.3_{-2.9}^{+5.8}$ and the best fit $n=1$ normalization for this case is $Q_{r m s-P S}=17.1_{-2.3}^{+2.5}$. These values along with corresponding results from Yr1 and Yr2 are given in Table 10.1. The relatively low value of the observed quadrupole in the

Table 10.1: $n$ and $Q_{\tau m s-P S}$ fits to the Correlation Function

\begin{tabular}{|rllll|}
\hline Data & $n$ & $Q_{r m s-P S}$ & $\chi^{2}$ & $\chi^{2} / D O F$ \\
\hline Yr1, NQ & $0.8_{-1.5}^{+1.0}$ & $18.9_{-6.8}^{+13.5}$ & 57 & 0.84 \\
$Q$ & $1.6_{-1.1}^{+0.6}$ & $12.7_{-3.8}^{+5.7}$ & 73 & 1.07 \\
\hline Yr2, NQ & $1.1_{-1.2}^{+0.8}$ & $19.0_{-6.0}^{+10.1}$ & 59 & 0.87 \\
$\mathrm{Q}$ & $1.8_{-1.0}^{+0.6}$ & $13.3_{-3.5}^{+7.4}$ & 93 & 1.36 \\
\hline $2 \mathrm{Yr}, \mathrm{NQ}$ & $1.2_{-1.0}^{+0.6}$ & $17.3_{-5.1}^{+7.1}$ & 38 & 0.56 \\
$\mathrm{NQ}$ & $\equiv 1.0$ & $18.6_{-2.4}^{+3.2}$ & 39 & 0.57 \\
$\mathrm{Q}$ & $1.7_{-0.9}^{+0.5}$ & $13.3_{-2.9}^{+5.8}$ & 80 & 1.17 \\
$\mathrm{Q}$ & $\equiv 1.0$ & $17.1_{-2.3}^{+2.5}$ & 92 & 1.33 \\
\hline
\end{tabular}

DMR maps produces the difference between the quadrupole included (" $Q$ ") and quadrupole excluded ("NQ") results.

The $\chi^{2}$ fits of equation (10.3) have the virtue of simplicity but the correlation function models $C_{n}(\alpha)$ cannot account for the shifts in power due to the $20^{\circ}$ Galactic latitude cut, the numerator $\left(C_{d}-C_{n}\right)$ is not expected to be a perfect Gaussian (Cayón \& MartinezGonzalez 1991, White, Krauss \& Silk 1993) and the cosmic variances are correlated: $\sigma_{n}\left(\alpha_{i}\right)$ is not independent of the $\sigma_{n}$ from a different $\alpha$ bin (see Scaramella \& Vittorio 1993, Seljak 
\& Bertschinger 1993). Thus Monte Carlo calibration of this procedure is necessary to check its validity and obtain error bars. We simulated $2 \mathrm{Yr}_{\mathrm{r}} 53 \mathrm{GHz}$ and $90 \mathrm{GHz}$ sky maps with $n=1$ and $Q_{r m s-P S}=17 \mu \mathrm{K}$ structure, including cosmic variance, DMR noise and DMR sky coverage. We subtracted the best fit mean, dipole and quadrupole in the $|b|>20^{\circ}$ region and computed the cross-correlation function for the same region. We plugged this $C(\alpha)$ into equation (10.3) in place of $C_{d}(\alpha)$ and solved for $n$ and $Q_{r m s-P S}$ as described above. These simulations were used to estimate the confidence level contours of Figure 10.1 and projected onto the $n$ and $Q_{\tau m s-P S}$ axes, give the error bars quoted in Table 10.1 .

For 2000 simulations of $n=1$ and $Q_{r m s-P S}=17 \mu \mathrm{K}$, the output distribution of $n$ values is asymmetric with a longer tail in the low $n$ direction. The mean, rms, median and mode of the distribution are $0.91,0.77,1.1$ and 1.2 respectively. The output distribution of $Q_{r m s-P S}$ values is asymmetric with a longer tail in the high $Q_{r m s-P S}$ direction. The mean, rms, median and mode of the distribution are 20.7, 8.2, 19.0 and $14.0 \mu \mathrm{K}$ respectively. Notice that the input values are straddled by the mode and the mean of the distributions. If one uses mean values to estimate a bias one obtains an $n$ bias of $-0.09 \pm 0.02$ and a $Q_{r m s-P S}$ bias of $+3.7 \pm 0.2 \mu \mathrm{K}$. One could argue that the median is a more robust indicator of central tendency when a distribution is skewed. Using median values one obtains an $n$ bias of $+0.1 \pm 0.02$ and a $Q_{r m s-P S}$ bias of $+2.0 \pm 0.2 \mu \mathrm{K}$. The $n$ and $Q_{\tau m s-P S}$ values in Table 10.1 do not include bias corrections.

The elongated error ellipse of Figure 10.1 is the result of the $n$ and $Q_{r m s-P S}$ degeneracy seen in Figure 4.2: increasing $n$ and increasing $Q_{\tau m s-P S}$ have a very similar effect on $C(\alpha)$, especially in the high signal to noise, small $\alpha$ region. The lines of degeneracy as determined respectively by this analysis, Adams et al.(1992), Seljak \& Bertschinger (1993), Smoot et al. (1994), Bennett et al. (1994) and Górski et al. (1994) are

$$
\begin{aligned}
& Q_{r m s-P S}=18.6 \exp [0.38(1-n)] \\
& Q_{r m s-P S}=14.9 \exp [0.31(1-n)] \\
& Q_{r m s-P S}=15.7 \exp [0.46(1-n)] \\
& Q_{r m s-P S}=15.7+6.6(1-n) \\
& Q_{r m s-P S}=18.2 \exp [0.58(1-n)] \\
& Q_{r m s-P S}=(19.08+0.95 n) \exp [0.74(1-n)]
\end{aligned}
$$

and are plotted in Figure 10.2. 


\subsection{Other Determinations of $n$ and $Q_{r m s-P S}$}

A variety of methods have been devised to extract $n$ and $Q_{\tau m s-P S}$ from the DMR data. Smoot et al. (1992) obtained $n$ and $Q_{\tau m s-P S}$ by fitting the correlation function of the DMR skymaps to equation (10.2) and to Monte Carlo simulations. Scaramella \& Vittorio (1993) also performed $\chi^{2}$ fits. Both of these fits used the Gaussian approximation to the DMR beam. A maximum likelihood method has been used by Seljak \& Bertschinger (1993) and Bennett et al. (1994) where the quantity minimized is

$$
-0.5 \ln (\mathcal{L})=\Delta C_{i} M_{i j}^{-1} \Delta C_{j}+\ln (\operatorname{det} \mathrm{M})
$$

where $\Delta C_{i}=C_{d}(i)-C_{n}(i)$ and the covariance matrix $\mathrm{M}$ was obtained by Monte Carlo simulations for a matrix of $n$ and $Q_{r m s-P S}$ values. Orthogonal functions on a Galacticplane-removed sphere were used to obtain power spectrum fits for $n$ and $Q_{r m s-P S}$ by Wright et al. (1994b) and by Górski et al. (1994). A technique involving RMS as a function of smoothing was used by Adams et al.(1992) and Smoot et al.(1994). A topological measure called the genus was used by Torres (1994) and Smoot et al. (1994).

Published values for $n$ and $Q_{r m s-P S}$ are listed in Table 10.2 and plotted in Figure 10.2. The best-fit quadrupole is sometimes removed before $n$ and $Q_{\tau m s-P S}$ are determined. Because of the low quadrupole in the DMR data and the degeneracy of $n$ and $Q_{r m s-P S}$ (see

* Figure 4.2), quadrupole included determinations are shifted to the lower right compared to quadrupole excluded determinations. This quadrupole shift depends on the technique used but is in the approximate range $[0.2,0.5]$ for $n$ and $[2,4] \mu \mathrm{K}$ for $Q_{r m s-P S}$. The three points in the lower right include the quadrupole. The variety of methods used to determine $n$ and $Q_{r m s-P S}$ yield consistent results (see Figure 10.2).

The $n$ values obtained are consistent with the prediction of inflation that $n \approx 1$. It is interesting to note, however, that an $n=1$ power spectra was discussed and motivated ten years before inflation (Harrison 1970, Zeldovich 1972, Peebles \& Yu 1970). Global monopoles and texture models for structure formation also seem to be consistent with $n \approx 1$ (Bennett \& Rhie 1993). 
Table 10.2: Other $n$ and $Q_{r m s-P S}$ Determinations from DMR Data

\begin{tabular}{|c|c|c|c|}
\hline Reference & Method & $n$ & $Q_{r m s-P S}[\mu \mathrm{K}]$ \\
\hline Smoot et al.(1992) & $\begin{array}{l}\text { Yr1,CF,NQ,GB,NCV,Yr1 } \\
\text { CV } \\
\text { all data,Q,CV }\end{array}$ & $\begin{array}{l}1.1 \pm 0.5 \\
\equiv 1 \\
1.15_{-0.65}^{+0.45} \\
1.0 \pm 0.6\end{array}$ & $\begin{array}{l}16 \pm 4 \\
16.7 \pm 4 \\
16.3 \pm 4.6 \\
17 \pm 5 \\
\end{array}$ \\
\hline Adams et al. (1992) & YI1,CF,GB & $\equiv 1$ & $14.9 \pm 0.5$ \\
\hline Scaramella \& Vittorio (1993) & Yr1,CF,GB & $\begin{array}{l}1.1 \\
\equiv 1\end{array}$ & $\begin{array}{l}14.2 \\
14.5 \pm 1.7\end{array}$ \\
\hline Seljak \& Bertschinger(1993) & $\begin{array}{l}\mathrm{Yr} 1, \mathrm{CF} \\
\mathrm{NQ} \\
\mathrm{Q}\end{array}$ & $\begin{array}{l}\equiv 1 \\
1.2 \\
0.9\end{array}$ & $\begin{array}{l}15.7 \pm 2.6 \\
14.8 \\
16.1\end{array}$ \\
\hline Wright et al. (1993) & $\begin{array}{l}\text { YI1,DMRB } \\
\text { GETSKYRMS } \\
\text { DMRSMUTH }\end{array}$ & $\begin{array}{l}\equiv 1 \\
\equiv 1 \\
\equiv 1\end{array}$ & $\begin{array}{l}\approx 15(<16.7) \\
\approx 16.7 \\
\approx 17.8\end{array}$ \\
\hline Torres (1994) & Yr1,G & $1.2 \pm 0.3$ & $\equiv 16$ \\
\hline Smoot et al.(1994) & $\begin{array}{l}\text { Yr1,G,Q,DMRB } \\
\text { RMS }\end{array}$ & $\begin{array}{l}1.7_{-1.1}^{+1.3} \\
1.7_{-0.6}^{+0.3}\end{array}$ & $\begin{array}{l}15.7 \pm 2.2 \\
13.2 \pm 2.5\end{array}$ \\
\hline$\overline{\text { Wright et al. (1994b) }}$ & $\begin{array}{l}\text { Yr } 1,0 F, N Q, D M R B \\
2 Y_{\mathrm{r}}, 3 \leqq \ell \leqq 30 \\
3 \leqq \ell \leqq 19 \\
53,3 \leqq \ell \leqq 30 \\
53+90 \\
\text { NG }\end{array}$ & $\begin{array}{l}1.69_{-0.52}^{+0.45} \\
1.25_{-0.45}^{+0.4} \\
1.46_{-0.44}^{+0.41} \\
\equiv 1 \\
\equiv 1 \\
\equiv 1\end{array}$ & $\begin{array}{l}- \\
- \\
- \\
20.8 \pm 2.8 \\
19.8 \pm 2.0 \\
16.1 \pm 3.3\end{array}$ \\
\hline Bennett et al. (1994) & $\begin{array}{l}2 \mathrm{Yr}_{\mathrm{r}, \mathrm{CF}, \mathrm{DMRB}, \mathrm{Q}} \\
\text { marginal } \mathrm{n} \\
\mathrm{NQ} \\
\text { marginal } \mathrm{n}\end{array}$ & $\begin{array}{l}1.42_{-0.55}^{+0.49} \\
\equiv 1 \\
1.42 \pm 0.37 \\
1.11_{-0.55}^{+0.60} \\
\equiv 1 \\
1.11 \pm 0.40\end{array}$ & $\begin{array}{l}14.3_{-3.3}^{+5.2} \\
18.2 \pm 1.5 \\
- \\
17.4_{-5.2}^{+7.5} \\
18.6 \pm 1.6 \\
-\end{array}$ \\
\hline Górski et al.(1994) & $\begin{array}{l}2 \mathrm{Yr}_{\mathrm{r}, \mathrm{OF}, \mathrm{DMRB}, \mathrm{Q}} \\
\text { marginal } \mathrm{n} \\
\mathrm{NQ} \\
\text { marginal n }\end{array}$ & $\begin{array}{l}1.22_{-0.52}^{+0.46} \\
\equiv 1 \\
1.10 \pm 0.32 \\
1.02_{-0.59}^{+0.53} \\
\equiv 1 \\
0.87 \pm 0.36\end{array}$ & $\begin{array}{l}17.0_{-4.8}^{+7.6} \\
19.9 \pm 1.6 \\
- \\
20.0_{-6.5}^{+10.5} \\
20.4 \pm 1.7 \\
-\end{array}$ \\
\hline Banday et al. (1994) & 2Yr,RMS,DMRB & $\equiv 1$ & $19.4_{-2.1}^{+2.3}$ \\
\hline
\end{tabular}

Notes: CF: Correlation Function, OF: Orthogonal Functions, G: Genus, NQ: No Quadrupole, Q: Quadrupole Included, GB: Gaussian Beam, DMRB: DMR Beam, CV: Includes Cosmic Variance, NCV: No Cosmic Variance Included 


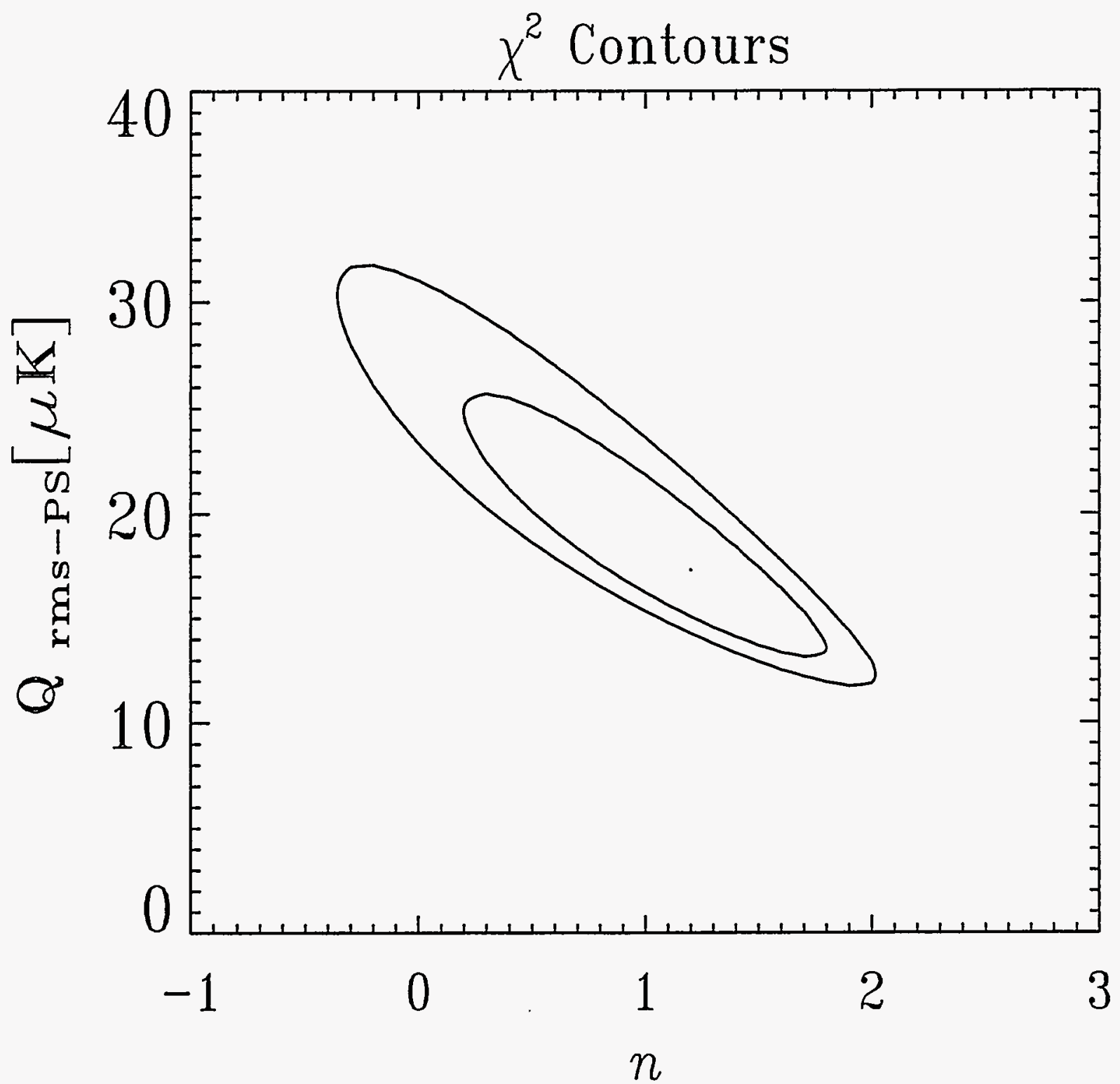

Figure 10.1: $\chi^{2}$ contours in $n, Q_{r m s-P S}$ space of fits to the $2 \mathrm{YT}_{\mathrm{T}} \mathrm{A}+\mathrm{B} 53 \mathrm{GHz} \times 90 \mathrm{GHz}$ correlation function. The best fit is marked by the dot at $n=1.2$ and $Q_{r m s-P S}=17.3 \mu \mathrm{K}$. Contours correspond to the $68 \%$ and $95 \%$ confidence regions. 


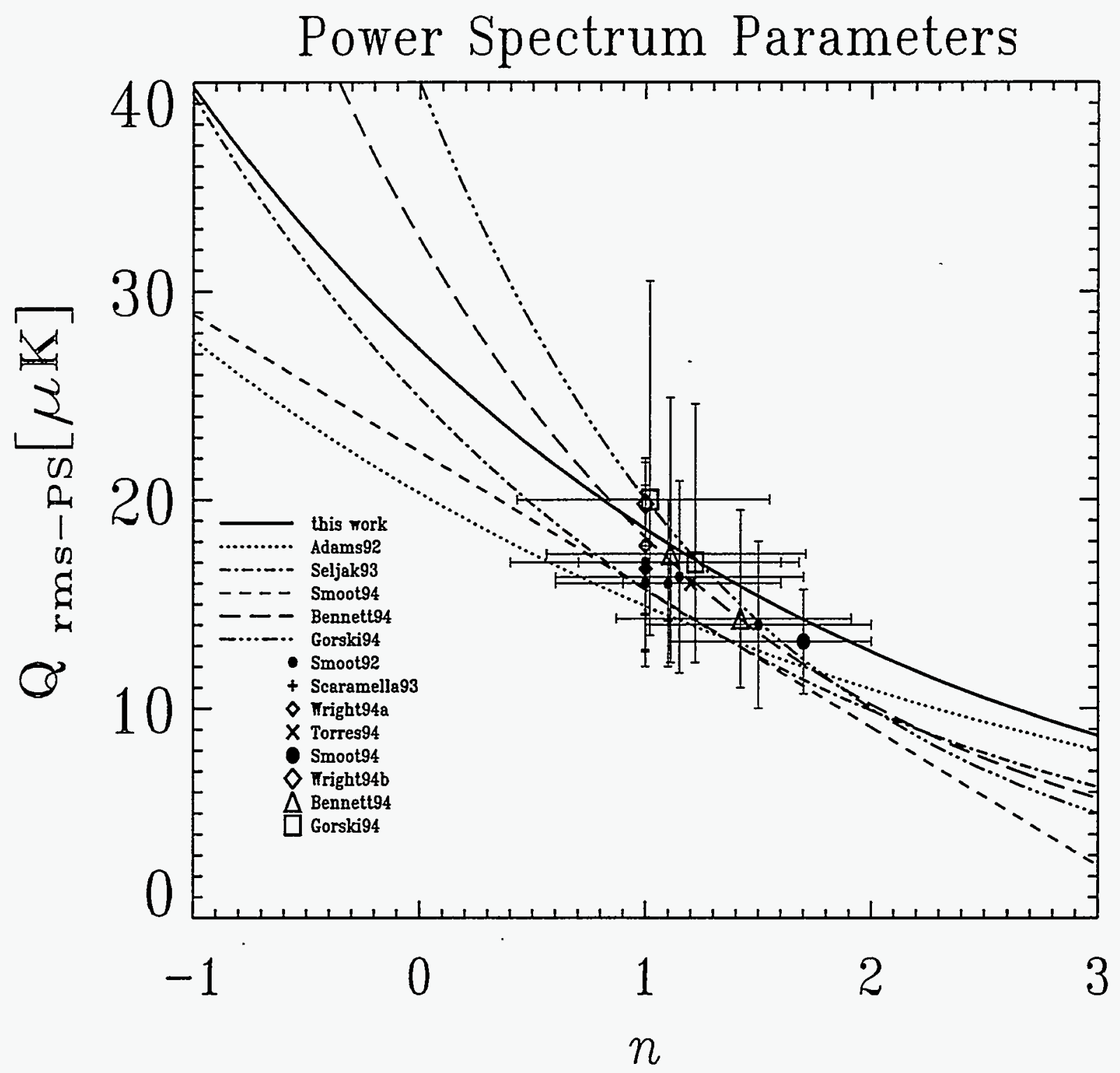

Figure 10.2: Determinations of Power Spectrum Parameters $n$ and $Q_{r m s-P S}$. The best-fit quadrupole is sometimes removed before $n$ and $Q_{r m s-P S}$ are determined. Because of the low $Q_{r m s}$ in the DMR data and the degeneracy of $n$ and $Q_{r m s-P S}$ (see Figure 4.2), quadrupole excluded determinations are shifted to the upper left compared with quadrupole included determinations. The three points in the lower right include the quadrupole. 


\title{
Chapter 11
}

\section{Conclusions and the Future}

The history of cosmology shows us that in every age devout people believe that they have at last discovered the true nature of the Universe.

(E. Harrison, Cosmology 1981)

\begin{abstract}
We summarize the thesis and draw useful conclusions about further analysis. We discuss the experimental assault on the power spectrum of CMB fluctuations.
\end{abstract}

\subsection{Conclusions}

In this thesis we have presented in detail a two-point correlation function analysis which helped lead to the DMR discovery of CMB anisotropies. The Galactic latitude cut independence of the correlation function is strong evidence that the signal is not Galactic in origin. As expected of a CMB signal, the correlation function is consistent with no frequency dependence. The statistical significance of the structure seen in the correlation function of the first, second and two year maps is respectively $>9 \sigma,>10 \sigma$ and $>18 \sigma$ above the noise. A simple $\chi^{2}$ fit of the model power spectra parameters $n$ and $Q_{r m s-P S}$ to the correlation function yields $n=1.2_{-1.0}^{+0.6}$ and $Q_{r m s-P S}=17.3_{-5.1}^{+7.1}$.

The noise in the DMR sky maps was found to be correlated at a low level. The structure of the pixel temperature covariance matrix was determined. The noise covariance matrix of a DMR sky map is diagonal to an accuracy of better than $1 \%$. Thus the noise in the 
DMR maps can be well approximated as noise from a single-beam experiment. Previously published DMR results are not significantly affected by correlated noise.

\subsection{Further DMR Analysis}

After completing four years of successful operation, the monitoring of the DMR instruments was stopped as scheduled in the last week of December, 1993. The two-year DMR data was released to the public in June, 1994, and the full four-year data set is scheduled to be released in 1995 . The consistency of years 3 and 4 with the first two years will be checked.

The correlation function will continue to play an important role in both the DMR analysis and in the comparison of the DMR maps with other data sets. As more data is included the noise contribution to the error bars goes down as $1 /$ time but the unavoidable cosmic variance remains constant. Cross-correlations with other data sets are not limited by cosmic variance and so benefit correspondingly more from a larger signal to noise ratio.

The DMR team has published about two dozen papers based on the DMR data (Appendix A). Papers in preparation include cross-correlations of the DMR data with other data sets, isolation and definition of particular spots in the maps and polarization limits derived from the data.

\subsection{Experimental Assault on CMB Anisotropies}

Table 11.1 is a compilation of recent CMB anisotropy experiments. The range of angular scales is from $\ell=2$ to several thousand. The main goal of these experimental efforts is to confirm or refute the DMR results at large angular scales and to determine the complete CMB power spectrum at a high enough accuracy to rule out some contending models and large chunks of parameter space. The data on small angular scales is more comparable with the structures we see around us. In contrast to the clean normalization at DMR scales, small angular scale anisotropies are strongly model dependent. The DMR normalization supports the need for dark matter but cannot say exactly how much or what kind. Small scale anisotropy experiments should be able to discriminate between hot and cold dark matter and may provide a more direct detection of dark matter.

A knowledge of the complete power spectrum will answer questions like: Is there a 
Doppler peak? At what angular scale? Are the perturbations only scalar or are there also tensor perturbations? At what angular scale does the finite thickness of the surface of last scattering cut off the anisotropies? Was there an epoch of reionization? At what level is the CMB polarized? Can the shape of the inflaton potential be determined by the CMB power spectrum?

\subsubsection{Confirmation}

The DMR results have been most directly confirmed by the Ganga et al. results (1994) from the balloon-borne $170 \mathrm{GHz}$ FIRS experiment. It covered $\approx 25 \%$ of the sky but much of it was in the Galactic plane. Further flights of the same instrument to cover the rest of the sky will soon complete the full-sky survey. Another experiment with a comparable angular size is Tenerife. A dozen detections at small angular scales have recently been announced all at approximately the level expected from the DMR normalization. These detections can therefore be construed as indirect confirmations of the DMR result. The Russian instrument RELICT II may be launched next year or in 1996. It should have the sensitivity to check, refine or refute the DMR result most directly.

\subsubsection{Small Angular Scales}

One of the main goals of the small angular scale experiments is to locate the expected large Doppler peak. This will be a major milestone on the way to determining the power spectrum in the range $50<\ell<f e w \times 1000$. There are now about a dozen groups trying to focus in on the small angular scale anisotropies. The proposed European Space Agency COBRAS/SAMBA flight ( 2001) may have something to say about half degree anisotropies over a large fraction of the sky.

Progress in CMB research is hindered by our ignorance of the Galactic and extragalactic foregrounds at radio, millimetric and IR frequencies (Banday 1991). Multifrequency maps of the Galaxy are needed to identify and subtract these unwanted effects from CMB data (DeAmici et al. 1993). Radio galaxies seem to be particularly problematic foreground for small scale experiments.

The range of angular scales in Table 11.1 is from $\ell=2$ to several thousand. Notice the string of upper limits on the smallest angular scales while in the half degree to DMR scales there have been many claimed detections. The detections of small angular scale measurements refer to the $\Delta T / T$ from $\mathrm{C}(0)$ of a Gaussian auto-correlation model. The 
beam size of the instrument is not usually the angular scale of the best $\Delta T / T$ detection or limit. The results quoted are for the coherence angle in parenthesis, not the actual size of beam. The results do not differ by more than a factor of two or three.

There is a wide range of workers building a coherent picture of structure formation from Galaxy surveys $(\delta \rho / \rho)$, from bulk velocity determinations ( $\left.V_{b u l k}\right)$ and from CMB anisotropies. We have seen in Section 4.3 how the power spectrum of mass over-densities can be related to the large scale power spectrum of CMB fluctuations. The level of CMB anisotropy can also be predicted by assuming that the velocity distribution of field galaxies is the direct result of over-densities still in the linear regime. In fact, fairly accurate predictions of the anisotropy normalization amplitude $Q_{r m s}-P S$ were made for example by Abbott and Wise (1984), Silk (1986), Górski (1991) and Scaramella (1992). This close relationship between CMB fluctuations $\delta T / T$, mass fluctuations $\delta \rho / \rho$, and bulk velocities $V$ is depicted in Figure 11.1. The process of piecing the data together to form a consistent picture of structure formation can be called closure.

The DMR discovery of large scale inhomogeneities verifies the basic gravitational instability picture of structure formation. The evolution of structure is being tracked down. Cosmology seems to be making progress. However the DMR normalization level of CMB anisotropy seems to require the existence of non-baryonic dark matter (Wright et al. 1992). The question of the existence and nature of dark matter appears increasingly urgent. The recent additions of inflation and dark matter to the big bang model are beginning to be tested.

\subsection{New Field of Astronomy}

Until the COBE DMR detection, the absence of anisotropy in the CMB was taken to be one of the important failings of the big bang model (Narlikar 1983). In 1980 Burbridge pointed out, "If no fluctuations can be found, we have no direct evidence at all that galaxies are formed at early epochs through gravitational instability". Thus the DMR discovery of $\mathrm{CMB}$ anisotropies can be interpreted as direct evidence that galaxies formed through gravitational instability. If the DMR results stand, a whole new class of objects has been found. Along with quasars, pulsars and black holes we now have hot and cold spots on the surface of last scattering. Bumps, spots, pips and blobs are now being analyzed as individual objects, not just being averaged over to determine the statistical level of fluctuation (e.g. 
correlation functions). Their study and detailed characterization will establish a new branch of astronomy. There will be catalogs with names and numbers. The DMR maps have pushed us into the "bump" era of CMB cosmology.

\section{Closure}

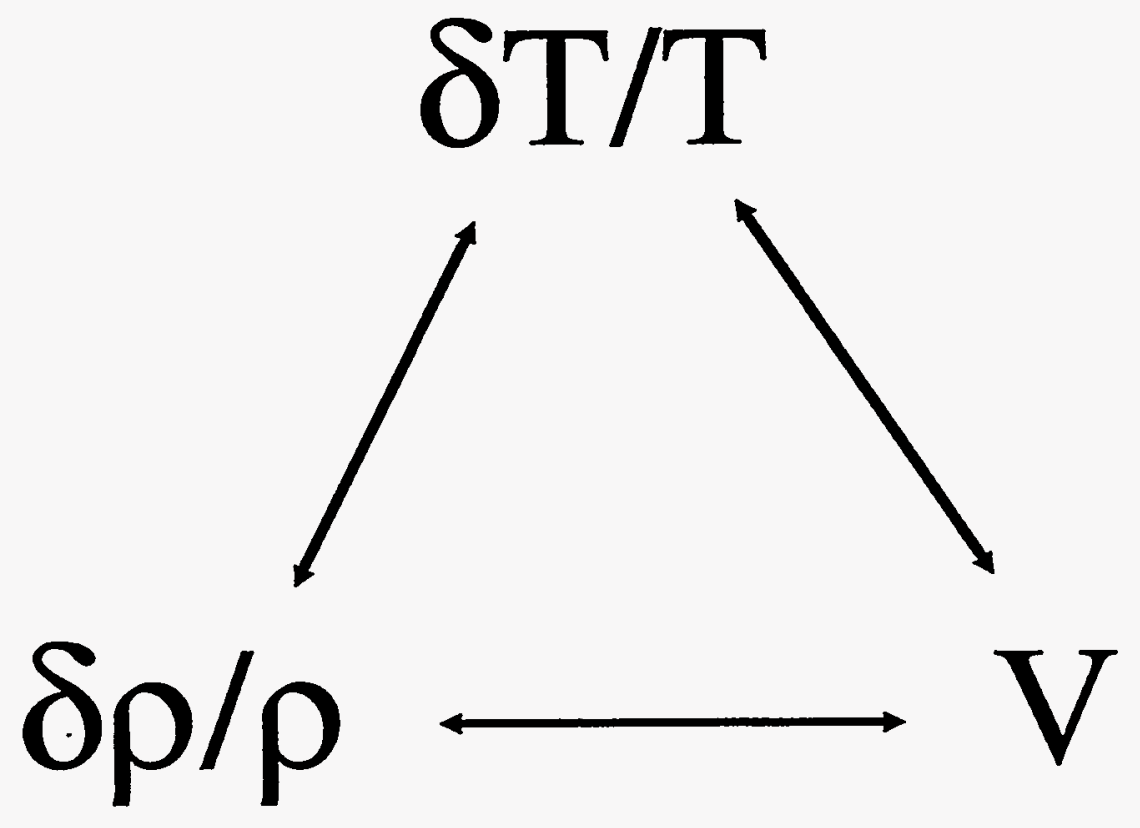

Figure 11.1: Closure. The data from CMB fluctuations $(\delta T / T)$, mass fluctuations $(\delta \rho / \rho)$ and bulk velocities $(V)$ are closely linked. The process of piecing the data together to form a consistent picture of structure formation can be called closure. 
Table 11.1: Recent CMB Anisotropy Experiments

\begin{tabular}{|c|c|c|c|c|}
\hline Experiment & $\begin{array}{l}\text { Beam }{ }^{a} \\
\left(^{\circ}\right)\left(\theta_{c}, \ell_{e f f}\right) \\
\text { Chop, Sub }\end{array}$ & $\begin{array}{l}\text { Frequency } \\
(\mathrm{GHz})\end{array}$ & $\begin{array}{l}\text { Result }^{b} \\
{\left[\Delta T / T \times 10^{-6}\right] \mathrm{CL}}\end{array}$ & $\begin{array}{l}\text { Reference } \\
\text { Coverage/Date }\end{array}$ \\
\hline $\begin{array}{l}\text { DMR } \\
\text { satellite }\end{array}$ & $\begin{array}{l}7^{\circ}\left(13.5^{\circ}, 6\right) \\
60^{\circ}, \mathrm{D}^{*}\end{array}$ & $\begin{array}{l}315390 \\
\text { mixer }\end{array}$ & $\begin{array}{l}{[16 \pm 3]} \\
{[11 \pm 1] @ 10^{\circ}}\end{array}$ & $\begin{array}{l}\text { Bennett94 } \\
100 \% 89-93\end{array}$ \\
\hline $\begin{array}{l}\text { Relikt } 1 \\
\text { satellite }\end{array}$ & $\begin{array}{l}5.8^{\circ}(6) \\
90^{\circ}, \mathrm{D}^{*}\end{array}$ & $\begin{array}{l}37 \\
\text { para amp }\end{array}$ & {$[<73] 90 \%$} & $\begin{array}{l}\text { Strukov88 } \\
85 \%, 83-84\end{array}$ \\
\hline $\begin{array}{l}\text { FIRS/MIT } \\
\text { balloon }\end{array}$ & $\begin{array}{l}3.8^{\circ}(20) \\
0^{\circ}, D^{*}\end{array}$ & $\begin{array}{l}170230480680 \\
\text { bolometer }\end{array}$ & {$[<16] 95 \%$} & $\begin{array}{l}\text { Meyer91 } \\
33 \% 89\end{array}$ \\
\hline $\begin{array}{l}\text { Tenerife } \\
\text { mountain }\end{array}$ & $\begin{array}{l}5.1^{\circ}\left(4^{\circ}, 23\right) \\
8.1^{\circ}, \mathrm{DD}\end{array}$ & $\begin{array}{l}101533 \\
\text { HEMT }\end{array}$ & {$\left[20_{-4}^{+5}\right]$} & $\begin{array}{l}\text { Hancock94 } \\
10 \%, 88-94\end{array}$ \\
\hline $\begin{array}{l}\text { SP91 } \\
\text { South Pole }\end{array}$ & $\begin{array}{l}1.4^{\circ}\left(1.5^{\circ}, 45\right) \\
3^{\circ}, \mathrm{D}\end{array}$ & $\begin{array}{l}26283134 \\
\text { HEMT }\end{array}$ & $\begin{array}{l}{\left[8_{-1}^{+2}\right]} \\
{[<14] 95 \%}\end{array}$ & $\begin{array}{l}\text { Schuster93 } \\
\text { Gaier92 } \\
<1 \% 91 \\
\end{array}$ \\
\hline $\begin{array}{l}\text { Saskatoon } \\
\text { ground }\end{array}$ & $\begin{array}{l}1.5^{\circ}\left(1.2^{\circ}, 55\right) \\
2.5^{\circ}, \mathrm{D}\end{array}$ & $\begin{array}{l}273033 \\
\text { bolometer }\end{array}$ & {$\left[15_{-5}^{+6}\right]$} & $\begin{array}{l}\text { Wollack94 } \\
<1 \% 92\end{array}$ \\
\hline $\begin{array}{l}\text { Argo } \\
\text { balloon }\end{array}$ & $\begin{array}{l}52^{\prime}\left(30^{\prime}, 70\right) \\
1.8^{\circ}, \mathrm{D}\end{array}$ & $\begin{array}{l}150250375600 \\
\text { bolometer }\end{array}$ & {$[14,30] 90 \%$} & $\begin{array}{l}\text { deBernardis } 94 \\
<1 \% 93\end{array}$ \\
\hline $\begin{array}{l}\text { Python } \\
\text { South Pole }\end{array}$ & $\begin{array}{l}50^{\prime}\left(1^{\circ}, 70\right) \\
2^{\circ} .75, \mathrm{DDD}\end{array}$ & $\begin{array}{l}100 \\
\text { bolometer }\end{array}$ & {$[30 \pm 10]$} & $\begin{array}{l}\text { Dragovan94 } \\
<1 \% 92-93\end{array}$ \\
\hline $\begin{array}{l}\text { MAX } \\
\text { balloon }\end{array}$ & $\begin{array}{l}40^{\prime}\left(25^{\prime}, 110\right) \\
1.3^{\circ}, \mathrm{D}\end{array}$ & $\begin{array}{l}90180270360 \\
\text { bolometer }\end{array}$ & $\begin{array}{l}{[<24] 95 \%} \\
{\left[42_{-11}^{+17}\right] 95 \%} \\
{\left[32_{-12}^{+15}\right] 95 \%} \\
{\left[35_{-16}^{+30}\right] 95 \%}\end{array}$ & $\begin{array}{l}\text { Meinhold93 } \\
\text { Gunderson93 } \\
\text { Clapp94 } \\
\text { Devlin94 } \\
<0.1 \% 92-93\end{array}$ \\
\hline $\begin{array}{l}\text { MSAM1 } \\
\text { balloon } \\
\text { MSAM2 }\end{array}$ & $\begin{array}{l}28^{\prime}\left(30^{\prime}, 100\right) \\
40^{\prime}, \mathrm{D} \\
18^{\prime}(200) \\
\mathrm{DD}\end{array}$ & $\begin{array}{l}170270500680 \\
\text { bolometer }\end{array}$ & $\begin{array}{l}{[6,22] 90 \%} \\
{[11,31] 90 \%}\end{array}$ & $\begin{array}{l}\text { Cheng94 } \\
<0.1 \% 6 / 92\end{array}$ \\
\hline $\begin{array}{l}\text { White Dish } \\
\text { South Pole }\end{array}$ & $\begin{array}{l}12^{\prime}\left(9^{\prime}, 300\right) \\
24^{\prime}, \mathrm{D}\end{array}$ & 90 & {$[<23] 95 \%$} & $\begin{array}{l}\text { Tucker93 } \\
<0.1 \% 93\end{array}$ \\
\hline $\begin{array}{l}\text { OVRO } \\
\text { valley }\end{array}$ & $\begin{array}{l}1.8^{\prime}\left(2.6^{\prime}, 1800\right) \\
7^{\prime}, \mathrm{DD} \\
\end{array}$ & $\begin{array}{l}20 \\
\text { radio telescope }\end{array}$ & {$[<19] 95 \%$} & $\begin{array}{l}\text { Readhead89 } \\
<0.1 \% 88\end{array}$ \\
\hline $\begin{array}{l}\text { OVRO RING } \\
\text { valley }\end{array}$ & $1.8^{\prime}(1800)$ & $\begin{array}{l}20 \\
\text { radio telescope }\end{array}$ & {$[<45] 95 \%$} & $\begin{array}{l}\text { Myers93 } \\
<0.1 \% 91 \\
\end{array}$ \\
\hline $\begin{array}{l}\text { VLA } \\
\text { ground }\end{array}$ & $18^{\prime \prime}\left(80^{\prime \prime}\right)$ & $\begin{array}{l}8.4 \\
\text { interferometer }\end{array}$ & {$[<19] 95 \%$} & $\begin{array}{l}\text { Fomalont } 93 \\
<0.1 \% 92\end{array}$ \\
\hline
\end{tabular}

a FWHM of beam, $\left(\theta_{c}\right.$ : coherence angle of the best fit Gaussian auto-correlation function, $\ell_{\text {eff }}$ : effective $\ell$ of experimental window function.)

$\mathrm{D}$ : single difference, $\mathrm{D}^{*}$ : single difference but equivalent to a single-beam experiment, DD: double difference, DDD: triple difference

${ }^{b}$ Results at the $\theta_{c}$ angular scale except for $D^{*}$ experiments whose results are at the FWHM given. 


\section{Bibliography}

[1] Abbott, L. F., \& Wise, M. B. 1984, ApJ, 282, L47

[2] Adams, F., Bond, J. R., Freese, K., Frieman, J., \& Olinto, A. 1992, Phys. Rev. D, 47, 426

[3] Alpher, R. A., Herman, R. 1948, Nature, 162, 774

[4] Anawalt, R. A., et al.1989, The Astronomical Almanac 1990, U. S. Gov. Print. Off.

[5] Arp, H. C., Burbidge, G., Hoyle, R., Narlikar, J. V., \& Wickramasinghe, N. C. 1990 Nature 346, 807

[6] Banday, A. J. 1991, Ph.D. Thesis, Univ. of Durham

[7] Banday, A. J., et al. 1994, ApJ, submitted

[8] Barrow, J. D., Juszkiewicz, R., Sonoda, D. H. 1985, MNRAS, 213, 917

[9] Bennett, C. L., et al. 1992a, ApJ, 391, 446

[10] Bennett, C. L., et al. 1992b, ApJ, 396, L7

[11] Bennett, C. L., et al. 1993, ApJ, 414, L77

[12] Bennett, C. L., et al. 1994, ApJ, 436, in press

[13] Bennett, D. P. \& Rhie, S. H. 1993, ApJ, 406, L7

[14] Bertschinger, E., Dekel, A., Faber, S. M., Dressler, A., Burstein, D. 1990, ApJ, 364, 370

[15] Bertschinger, E. 1992, in New Insights into the Universe, ed. V. J. Martinez, M. Portilla, \& D. Saez (Berlin:Springer) p. 65 
[16] Bertschinger, E. 1993, Berkeley CMB Workshop proceedings

[17] Boerner, G. 1988, The Early Universe, (Berlin:Springer)

[18] Boggess, N. W., et al. 1992, ApJ, 397, 420

[19] Bond, J. R., \& Efstathiou, G. 1987, MNRAS, 226, 655

[20] Bond, J. R., Efstathiou, G., Lubin, P. M., Meinhold, P. R. 1991, Phys. Rev. Lett., 66, 17,2179

[21] Bond, J. R. 1994, Astrophys. Lett. and Comm., in press

[22] Bond, J. R. 1994, Phys. Rev. D. submitted

[23] Bond, J. R. 1995, in Proc. of the IUCAA Dedication Ceremonies, Pune, India ed. Padmanabhan, T. (N.Y.:Wiley)

[24] Cayón, L., Martinez-Gonzalez, E., \& Sanz, J. L. 1991, MNRAS, 253, 599

[25] Chan, F. K., \& O'Neil, E. M. 1975, Computer Sciences Corp., EPRF Technical Report $2-75$

[26] Cheng, E. S., Cottingham, D.A., Fixsen, D.J., Inman, C.A., Kowitt, M. S., Meyer, S. S., Page, L. A., Puchalla, J. L., \& Silverberg, R. F. 1994, ApJ, 422, L37

[27] Clapp, A., et al. 1994, ApJ, submitted \& Steinhardt, P. J. 1993, PRL, 71, 324

[28] De Amici, G., et al. 1993, in Back to the Galaxy, AIP Conf. Proc. 278, ed. Holt, S. S., \& Verter, F. (N.Y.:AIP) p. 206

[29] de Bernardis, P., et al. 1994, ApJ, 422, L33

[30] Devlin, M. et al. 1994, ApJ, 430, L1

[31] Dicke, R. H., Peebles, P. J. E., Roll, P. G. \& Wilkinson, D. T. 1965, ApJ, 142, 414

[32] Dragovan, M., Ruhl, J., Novak, G., Platt, S. R., Crone, B., Peterson, J. B., \& Pernic, R. 1994, ApJ, preprint

[33] Dressler, A., et al. 1986, ApJ, 313, L37 
[34] Efstathiou, G. 1990, in Physics of the Early Universe, Proc. of the 36th Scottish Universities Summer School in Physics 1987. ed. Peacock, J., Heavens, A. F., Davies, A. T. (Edinburgh:SUSSP) p. 414

[35] Fich, M., Blitz, L., \& Stark, A. 1989, ApJ, 342, 272

[36] Fixsen, D. J., et al. 1994, ApJ, 420, 445

[37] Fomalont, E. B., et al. 1993, ApJ, 404, 8

[38] Gaier, T., Schuster, J., Gundersen, J., Koch, T., Seiffert, M., Meinhold, P., Lubin, P. 1992, ApJ, 398, L1

[39] Ganga, K., Cheng, E., Meyer, S., Page, L. 1993, ApJ, 410, L57

[40] Ganga, K., Page, L., Cheng, E., Meyer, S. 1994, APJL

[41] Górski, K. 1991, ApJ, 370, L5

[42] Górski, K. 1994, ApJ, 430, L85

[43] Górski, K., et al. 1994, ApJ, 430, L89

[44] Gunderson, J. O., et al. 1993, ApJ, 412, L1

[45] Hancock, S., Davies, R. D., Lasenby, A. N., Gutierrez de la Cruz, C. M., Watson, R. A., Rebolo R., \& Beckman, J. E. 1994, Nature, 367, 333

[46] Harrison, E. R. 1970, Phys. Rev. D1, 2726

[47] Harrison, E. R. 1981, Cosmology: The Science of the Universe, (New York:Cambridge Univ. Press)

[48] Harrison, E. R. 1987, Darkness at Night: A Riddle of the Universe, (Cambridge, Mass.:Harvard U. Press)

[49] Hinshaw, G., et al. 1994, ApJ, in press

[50] Hon, D. Computers in Physics, Sept/Oct 1991, p. 505

[51] Jackson, J. D., Classical Electrodynamics 1975, (N.Y.:Wiley) 
[52] Jackson, P. D., et al. 1991, in P.A.S.P. Conf. Series 25, Astronomical Data Analysis Software and Systems I, ed. D. M. Worrall, C. Biemesderfer, \& J. Barnes (San Francisco:ASP) 382

[53] Janssen, M. A. 1978, COBE Memo 4005, DMR: Sensitivity vs horn opening angle I.

[54] Janssen, M. A., \& Gulkis, S. 1992, in The Infrared and Submillimetre Sky After COBE, ed. M. Signore, \& C. Dupraz (Dordrecht:Kluwer), 391

[55] Janssen, M. A., Gulkis, S., Bennett, C. L., Kogut, A. J. 1994, in Infrared Spaceborne Remote Sensing Proc. of the SPIE conference, July 1993, San Diego

[56] Kaiser, N., \& Silk J. 1986, Nature, 324

[57] Keegstra, P. B., et al. 1991, in P.A.S.P. Conf. Series 25, Astronomical Data Analysis Software and Systems I, ed. D. M. Worrall, C. Biemesderfer, \& J. Barnes (San Francisco:ASP) 530

[58] Kerr, F. J., \& Lynden-Bell, D. 1986, MNRAS, 221, 1023

[59] Klypin, A. A., Sazhin, M. V. Strukov, I. A., \& Skulachev, D. P. 1987, Soviet. Astr. Lett., 13, 104

[60] Kneissl, R., \& Smoot, G. F., COBE Note 5053, July 10, 1993

[61] Kogut, A., Smoot, G.F., et al. 1992, ApJ, 401, 1

[62] Kogut, A., Lineweaver, C. H., et al. 1993, ApJ, 419, 1

[63] Kolb, E. W., \& Turner, M. S. 1990 The Early Universe, (Redwood City, CA:AddisonWesley) p. 505

[64] Kumar, V. K., Freedman, I., et al., preprint for Flight Mechanics/Estimation Theory Symposium, NASA/GSFC, May, 1992

[65] Lang, K. 1980, Astrophysical Formulae, (Berlin:Springer) p. 117

[66] Lauer, T. R., \& Postman, M. 1992, BAAS, 24, 1264

[67] Layzer, D. 1992, ApJ, 392, L5

[68] Liboff, R. L. 1980, Introductory Quantum Mechanics, (San Francisco:Holden-Day) 
[69] Lineweaver, C. H. 1993, in Proc. of 1992 Trieste Summer School in High Energy Physics and Cosmology, ICTP Series in Theoretical Physics, Vol 9, ed. Gava, E., Narain, K., Randjbar-Daemi, S., Sezgin, E., \& Shafi Q. (Singapore:World Scientific) p. 772

[70] Lineweaver, C. H., Smoot, G. F., Kogut, A., Tenorio, L. 1994a, Astrophys. Lett. and Comm., in press

[71] Lineweaver, C. H., et al. 1994b, ApJ, 436, in press

[72] Longair, M. S. 1992, High Energy Astrophysics 2nd. ed. Vol I. (Cambridge U. Press: Cambridge) p. $71 \mathrm{ff}$

[73] Mather, J. C., et al., ApJ, in press 1994

[74] Meinhold, P., et al. 1993, ApJ, 409, L1

[75] Meyer, S. S., Cheng, E. S., Page, L. A. 1991, ApJ, 371, L7

[76] Misner, C. W. 1968, ApJ, 151, 431

[77] Myers, S. T., Readhead, A. C. S., Lawrence, C. R. 1993, ApJ, 405, 8

[78] Narlikar, J. V. 1983, Introduction to Cosmology, (Boston:Jones\&Bartlett) p. 394

[79] NASA, Cosmology Data Analysis Center (CDAC), DMR Software User's Guide Version 6.1 May 1994

[80] NASA, Cosmology Data Analysis Center (CDAC), DMR Project Pipeline Version 1.0 May 1994

[81] NASA, Cosmology Data Analysis Center (CDAC), DMR Science Pipeline Version 1.0 May 1994

[82] Padmanabhan, T. 1993, Structure Formation in the Universe, (Cambridge:Cambridge Univ. Press)

[83] Peebles, P. J. E., \& Wilkinson, D. T. 1968, Phys. Rev., 174, 2168

[84] Peebles, P. J. E., \& Yu, J. T. 1970, ApJ, 162, 815

[85] Peebles, P. J. E. 1973 Ap.J. 185, 413 
[86] Peebles, P. J. E., Schramm, D. N., Turner E. L., \& Kron R. G. 1991, Nature 352, 769

[87] Peebles, P. J. E. 1993, Principles of Physical Cosmology, (N.J.:Princeton) p. 134

[88] Penzias, A. A., Wilson, R. W. 1965, ApJ, 142, 419

[89] Press, W. H., Teukolsky, S. A., Vetterling, W. T., Flannery, B. P. 1992, Numerical Recipes, second edition, (Cambridge:Cambridge Univ. Press)

[90] Readhead, A. C. S., et al. 1989, ApJ, 346, 566

[91] Sachs, R. K., \& Wolfe, A. M. 1967, ApJ, 147, 73

[92] Scaramella, R., et al. 1989, Nature, 338,562

[93] Scaramella; R., \& Vittorio, N. 1990, ApJ, 353, 372

[94] Scaramella, R. 1992, ApJ, 390, L57

[95] Scaramella, R., \& Vittorio, N. 1993, MNRAS, 263, L17

[96] Schuster, J., et al. 1993, ApJ, 412, L47

[97] Seljak, U., \& Bertschinger E. 1993, ApJ, 417, L9

[98] Silk, J. 1986, in Inner Space/Outer Space ed. Kolb, E. W., Turner, M. S., Lindley, D., Olive, K., Seckel, P. (Chicago:Univ of Chicago Press) p. 143

[99] Silk, J. 1989, The Big Bang, (N.Y.:Freeman) p. 428

[100] Smoot, G. F., et al. 1990, ApJ, 360, 685

[101] Smoot, G. F., et al. 1991 ApJ, 371, L1

[102] Smoot, G. F., et al. 1992, ApJ, 396, L1

[103] Smoot, G. F., et al. 1994, ApJ

[104] Smoot, G. F., et al. 1994, J. Roy. Astron. Soc. Can., 88, 2

[105] Songailia, A., Cowie, L. L., Hogan, C. J., Rugers, M. 1994, Nature, 368, 599

[106] Stebbins, A. 1993 in 1993, in 16th Texas Symp. in Relativistic Astrophysics, Ann. N.Y. Acad. Sci., Vol. 688,824 
[107] Strauss, M., et al. 1992, ApJ, 397, 395

[108] Strukov, I. A., \& Skulachev, D.P. 1988, Astrophysics \& Space Physics Review, 6, 147

[109] Sunyaev, R. A., \& Zel'dovich, Ya. B. 1972, Comments AP. Space Sci., 4, 173

[110] Torres, S., et al. 1989, in Data Analysis in Astronomy, ed. V. di Gesù, L. Scarsi, P. Crane, J. H. Friedman, S. Levialdi, \& M. C. Maccarone (New York:Plenum), 319

[111] Torres, S. 1994, ApJ, 423, L9

[112] Tucker, G. S., et al. 1993, ApJ, 419, L45

[113] Vishniac, E. T. 1987, ApJ, 322, 597

[114] White, M., Krauss, L. M., Silk, J. 1993, ApJ, 418, 535

[115] White, M., Scott, D., \& Silk, J. 1994, ARAA, in press

[116] White, R. A., \& Stemwedel, S. W. 1992, in Astronomical Data Analysis Software and Systems I, ed. D. M. Worrall, C. Biemesderfer, \& J. Barnes (San Francisco:ASP) p. 379

[117] Wilson, M. L., \& Silk, J. 1981, ApJ, 243, 14

[118] Wollack, E. J. 1994, ApJ, 419, L49

[119] Wright, E. L., et al. 1991a, ApJ, 381, 200

[120] Wright, E. L., et al. 1991b, in After the First Three Minutes, AIP Conf. Proc. 222, ed. Holt, S. S., Bennett, C. L., Trimble, V. (N.Y.:AIP) p. 179

[121] Wright, E. L., et al. 1992, ApJ, 396, L13

[122] Wright, E. L. 1993, in 16th Texas Symp. in Relativistic Astrophysics, Ann. N.Y. Acad. Sci., Vol. 688,836

[123] Wright, E. L. 1994, in Proc. of the BCSPIN Puri Winter School, in press

[124] Wright, E. L., Smoot, G. F., Kogut, A., Hinshaw, G., Tenorio, L., Lineweaver, C., Bennett, C. L., \& Lubin, P. M. 1994a, ApJ, 420, 1

[125] Wright, E. L., et al. 1994b, ApJ, 436, in press 
[126] Yahil, A., Tammann, G. A., \& Sandage, A. 1977, ApJ, 217, 903

[127] Zeldovich, Y. B. 1972, MNRAS, 160, 1 


\title{
Appendix A
}

\section{DMR Publications}

\author{
Coauthored DMR Publications
}

COBE Differential Microwave Radiometers: Calibration Techniques

Bennett, C. L., Smoot, G. F., et al. 1992, ApJ, 391, 446

Structure in the COBE Differential Microwave Radiometer First Year Maps

Smoot, G. F., Bennett, C. L., et al. 1992, ApJ, 396, L1

Interpretation of the Cosmic Microwave Radiation Anisotropy Detected by the COBE Differential Microwave Radiometer

Wright, E. L., Meyer, S. S., et al. 1992, ApJ, 396, L13

COBE Differential Microwave Radiometers (DMR): Preliminary Systematic Error Analysis

Kogut, A., Smoot, G. F., et al. 1992, ApJ, 401, 1

Dipole Anisotropy in the COBE Differential Microwave Radiometers First-Year Sky Maps

Kogut, A., Lineweaver, C., et al. 1993, ApJ, 419, 1

Comments on the Statistical Analysis of Excess Variance in the COBE Differential Microwave Radiometer Maps

Wright, E. L., Smoot, G. F., et al. 1994, ApJ, 420, 1

Limits on Three-Point Correlations in the COBE DMR First Year Anisotropy Maps

Hinshaw, G., Kogut, A., et al. 1994, ApJ, in press 
The Cosmic Microwave Background Dipole Anisotropy: Testing the Standard Model

Lineweaver, C. H., Smoot, G. F., et al.1994, Astrophys. Lett. and Comm., in press

Cosmic Temperature Fluctuations from Two Years of COBE DMR Observations

Bennett, C. L., Kogut, A., et al. 1994, ApJ, 436, in press

Correlated Noise in the COBE DMR Sky Maps

Lineweaver, C. H., Smoot, G. F., et al. 1994, ApJ, 436, in press

On the RMS Anisotropy at $7^{\circ}$ and $10^{\circ}$ Observed in the COBE-DMR Two Year Sky Maps

Banday, A. J., Górski, K. M., et al. 1994, ApJ, submitted

\section{Conference Proceedings}

COBE Differential Microwave Radiometer (DMR) Data Processing Techniques Jackson, P. D., Smoot, G. F., Bennett, C. L., Aymon, J., Backus, C., DeAmici, G., Hinshaw, G., Keegstra, P. B., Kogut, A., Lineweaver, C., Rokke, L. A., Tenorio, L. 1991, in P.A.S.P. Conf. Series 25, Astronomical Data Analysis Software and Systems I, ed. D. M. Worrall, C. Biemesderfer, \& J. Barnes (San Francisco:ASP) 382

Daily Quality Assurance Software for a Satellite Radiometer System

Keegstra, P. B., Smoot, G. F., Bennett, C. L., Aymon, J., Backus, C., DeAmici, G., Hinshaw, G., Jackson, P. D., Kogut, A., Lineweaver, C., Rokke, L. A., Santana, J. 1991, in P.A.S.P. Conf. Series 25, Astronomical Data Analysis Software and Systems I, ed.

D. M. Worrall, C. Biemesderfer, \& J. Barnes (San Francisco:ASP) 530

The COBE Differential Microwave Radiometer Anisotropy Detection

Lineweaver, C. H. 1993, in Proc. of 1992 Trieste Summer School in High Energy Physics and Cosmology ICTP Series in Theoretical Physics, Vol 9, ed. E. Gava, K. Narain, S. Randjbar-Daemi, E. Sezgin, \& Q. Shafi (World Scientific:Singapore) p. 722

Comments on the COBE DMR Quadrupole Estimation

Tenorio, L., Smoot, G. F., Lineweaver, C., Hinshaw, G., Banday, A.

1994, Proceedings of the Santander Workshop on the Present and Future of the Cosmic Microwave Background 
Other Recent DMR Publications

Non-Cosmological Signal Contributions to the COBE DMR Anisotropy Maps Bennett, C. L., Hinshaw, G., et al. 1994, ApJ, in press

Search for Unresolved Sources in the COBE-DMR Two-Year Sky Maps

Kogut, A., Banday, A. J., et al. , 1994 ApJ, submitted

Angular Power Spectrum of the Microwave Background Anisotropy seen by the COBE Differentail Microwave Radiometer

Wright, E. L., Smoot, G. F., et al. 1994, ApJ, 436, in press

On Determining the Spectrum of Primordial Inhomogeneity from the COBE DMR Sky Maps: I. Method

Górski, K. M. 1994, ApJ, 430, L85

On Determining the Spectrum of Primordial Inhomogeneity from the COBE DMR Sky Maps: II. Results of Two Year Data Analysis

Górski, K. M., Hinshaw, G., et al. 1994, ApJ, 430, L89

COBE Differential Microwave Radiometers: Instrument Design and Implementation

Smoot, G. F., Bennett, C., et al. 1991, ApJ, 354, L37

Preliminary Results from the COBE Differential Microwave Radiometers: Large-Angular-Scale Isotropy of the Cosmic Microwave Background

Smoot, G. F., Bennett, C. L., et al. 1991, ApJ, 371, L1

Preliminary Separation of Galactic and Cosmic Microwave Emission for the COBE Differential Microwave Radiometer

Bennett, C. L., Smoot, G. F., et al. 1992, ApJ, 396, L7

Statistics and Topology of the COBE DMR First Year Sky Maps

Smoot, G. F., Tenorio, L., et al. ApJ, in press

The COBE Mission: Its Design and Performance Two Years After Launch

Boggess, N. W., Mather, J. C., et al. 1992, ApJ, 397, 420

DMR Software User's Guide

Version 6.1 May, 1994, NASA, Cosmology Data Analysis Center (CDAC)

DMR Project Pipeline

Version 1.0 May, 1994, NASA, Cosmology Data Analysis Center (CDAC)

DMR Science Pipeline

Version 1.0 May 1994, NASA, Cosmology Data Analysis Center (CDAC) 
Feasibility Study of a Quadrilateralized Spherical Cube Earth Data Base Chan, F. K., O'Neill, E. M. 1975, Computer Sciences Corp., EPRF Technical Report 2-75 Extended Studies of a Quadrilateralized Spherical Cube Earth Data Base O'Neill, E. M., \& Laubscher, R. E., 1976, Computer Sciences Corporation, NEPRF Technical Report 3-76 Prepared for the Naval Environmental Protection Research Facility, Monterey, California 


\section{Appendix B}

\section{Thesis Notation}

$i, j, k, \ell:$ indices of map pixels, $1 \leqq i, j, k, \ell \leqq N_{\text {pix }}$

$N_{\text {pix }}$ : number of pixels in.a DMR map, usually $N_{\text {pix }}=6144$

$m$ : index of differential measurements, $1 \leqq m \leqq m_{\text {tot }}$

$m_{\text {tot }}$ : total number of measurements for the channel under consideration, $m_{t o t} \approx 2 x 10^{8}(=$ $\left.6 x 10^{7} / \mathrm{yr} \times 4 \mathrm{yrs}\right)$

$\vec{T}$ : model temperature map of the CMB sky, $N_{\text {pix }}$ dimensional vector with components $T_{i}$ $\vec{T}_{o b s}$ : a DMR skymap, least squares estimate of $\vec{T}, N_{p i x}$ dimensional vector with components $T_{o b s, i}$ where $T_{o b s, i}=T_{i}+A_{i j}^{-1} \delta_{j}$

$\alpha_{i j}$ : is the separation angle between pixels $i$ and $j$

$\alpha$ : independent variable, argument of the correlation function $\mathrm{C}(\alpha)$.

$N_{i}$ : number of times pixel $i$ has been observed

$\bar{N}$ : average number of observations, $\frac{1}{N_{p i x}} \sum_{i=1}^{N_{p i x}} N_{i} \approx 17,500$ for one year maps

$N_{i j}$ : number of times pixel $i$ has been differenced with pixel $j$, approximated by $\frac{\left(N_{i}+N_{j}\right) / 2}{N_{r e f}}$ $\bar{N}_{i j}$ : average number of times that the central pixels are differenced with reference ring pixels, $\frac{1}{N_{\text {ref }} N_{\text {pix }}} \sum_{i, j} N_{i, j} \approx 64$

$w_{i}$ : weight given to $T_{o b s, i}$, usually $w_{i}=1 / \sigma_{i}^{2}=N_{i} / \sigma_{c h}^{2}$

$W(\alpha)=\sum_{i k}^{\alpha_{i k}=\alpha} w_{i} w_{k}$, if the weights equal one, then $W$ is the number of pixel pairs $i, j$ with $\alpha_{i j}$ within half a bin width of $\alpha$

$N_{\text {ref }}$ : average number of pixels in the reference ring of a given pixel, $\approx 273$

$\Delta_{m}$ : detector noise in the $m$ th differential measurement; comes from a Gaussian distribution of zero mean and variance $\sigma_{m}^{2}$ $\sigma_{m}^{2}$ : channel (and measurement) dependent variance of a given differential measurement 
$\sigma_{c h}^{2}$ : channel (but not measurement) dependent variance of the differential measurements $\sigma_{e f f}^{2}$ : the effective variance of the temperatures in a combined map, i.e., $A+B$, or the weighted average of $53+90$

$\sigma_{i}^{2}$ : the variance of pixel temperature $T_{o b s, i}$, usually $\sigma_{i}^{2}=\sigma_{c h}^{2} / N_{i}$

$n_{i}^{\text {tot}:}$ total noise in map temperature $T_{o b s, i}$

$n_{i}$ : approximation and leading term of $n_{i}^{t o t}$, usually assumed to come from a Gaussian of zero mean and variance $\sigma_{i}^{2}=\sigma_{c h}^{2} / N_{i} \cdot n_{i}=A_{i i}^{-1} \delta_{i}=\delta_{i} / N_{i}$

$D(m): m$ th calibrated and corrected differential measurement

$\mathrm{V}: m_{\text {tot }}$ by $N_{\text {pix }}$ matrix containing the pointing information; Numerical Recipes calls this the design matrix of the normal equations

$\vec{\delta}: \quad N_{p i x}$ dimensional vector, $\vec{\delta}=\mathrm{V}^{T} \vec{\Delta}$, a component $\delta_{i}$ is a random walk of differential measurement errors, from a Gaussian distribution of zero mean and variance $\sigma_{\delta_{i}}^{2}=N_{i} \sigma_{c h}^{2}$. A: $N_{p i x}$ by $N_{p i x}$ dimensional normal equations matrix referred to as the cross-products matrix, the moment matrix or curvature matrix. $A=\mathbf{V}^{T} \mathbf{\Sigma V}$ and sometimes redefined to the dimensionless $\left(=\mathrm{V}^{T} \mathrm{~V}\right) \cdot A_{i j}=-\frac{1}{2} \frac{\partial x^{2}}{\partial T_{i} \partial T_{j}}$.

$\mathrm{A}^{-1}$ : inverse of $\mathrm{A}, N_{p i x}$ by $N_{p i x}$ dimensional covariance matrix or error matrix of the fitted temperatures; $A_{i j}^{-1}=<\delta T_{o b s, i} \delta T_{o b s, j}>$

$\vec{M}$ : measurement vector $=\mathrm{V}^{T} \mathbf{\Sigma} \vec{D}$. When combined with the dimensionless $\mathrm{A}$ we ignore the $\Sigma . \vec{M}$ can be decomposed into a signal and a noise term: $\mathrm{V}^{T} \Delta T+\mathrm{V}^{T} \Delta=\vec{T}+\mathrm{A}^{-1} \vec{\delta}$ $\Sigma: m_{\text {tot }}$ by $m_{\text {tot }}$ matrix. Each element in the $m$ th row equals $\frac{1}{\sigma_{m}^{2}}$ $\sum_{i k}^{\alpha_{i k}=\alpha}$ : sum over all pixel pairs $i k$ whose separation angle $\alpha_{i k}$ lies within half a bin width of $\alpha$

$C_{u}$ : uniformly weighted correlation function

$C_{w}$ : weighted correlation function

$C_{m i d}$ : correlation function of mean and dipole subtracted maps

$C_{m d q}$ : correlation function of mean, dipole and quadrupole subtracted maps 


\section{Appendix C}

\section{DMR Notation}

We expand the skymap temperatures in spherical harmonics

$$
T(\theta, \phi)=\sum a_{\ell m} Y_{\ell, m}(\theta, \dot{\phi})=\sum b_{\ell m} F_{\ell, m}(\theta, \phi)
$$

where the convention used for the spherical harmonics $Y_{\ell m}(\theta, \phi)$ and the real valued spherical harmonics $F_{\ell, m}(\theta, \phi)$ are defined in Appendix $\mathrm{H}$. The sky can be approximately written in terms of the monopole, dipole and quadrupole expressed in "unit" normalization

$$
\begin{aligned}
T(\alpha, \delta) & =T_{o}+D_{x} \cos \delta \cos \alpha+D_{y} \cos \delta \sin \alpha+D_{z} \sin \delta+Q_{1} \frac{1}{2}\left(3 \sin ^{2} \delta-1\right) \\
& +Q_{2} \sin 2 \delta \cos \alpha+Q_{3} \sin 2 \delta \sin \alpha+Q_{4} \cos ^{2} \delta \cos 2 \alpha+Q_{5} \cos ^{2} \delta \sin 2 \alpha
\end{aligned}
$$

where $\alpha$ and $\delta$ are right ascension and declination respectively. Also

$$
\begin{aligned}
D^{2} & \equiv D_{x}^{2}+D_{y}^{2}+D_{z}^{2} \\
Q_{r m s}^{2} & \equiv \frac{4}{15}\left(\frac{3}{4} Q_{1}^{2}+Q_{2}^{2}+Q_{3}^{2}+Q_{4}^{2}+Q_{5}^{2}\right)
\end{aligned}
$$

or in "rms" normalization

$$
\begin{aligned}
& D_{r m s}^{2}=\frac{1}{3} D^{2}=\frac{1}{4 \pi} \sum_{m=-1}^{1} b_{1, m}^{2} \\
& Q_{r m s}^{2}=\frac{1}{4 \pi} \sum_{m=-2}^{2} b_{2, m}^{2} .
\end{aligned}
$$


The conversions between the coefficients in equation (C.2) and the Legendre polynomial coeffcients $b_{\ell m}$ are

$$
\begin{aligned}
& T_{o}=\frac{1}{\sqrt{4 \pi}} b_{00} \\
& D_{x}=-\sqrt{\frac{3}{4 \pi}} b_{11} \\
& D_{y}=-\sqrt{\frac{3}{4 \pi}} b_{1-1} \\
& D_{z}=+\sqrt{\frac{3}{4 \pi}} b_{10} \\
& Q_{1}=+\sqrt{\frac{5}{4 \pi}} b_{20} \\
& Q_{2}=-\sqrt{\frac{15}{16 \pi}} b_{21} \\
& Q_{3}=-\sqrt{\frac{15}{16 \pi}} b_{2-1} \\
& Q_{4}=+\sqrt{\frac{15}{16 \pi}} b_{22} \\
& Q_{5}=+\sqrt{\frac{15}{16 \pi}} b_{2-2} .
\end{aligned}
$$

There is some variation in the notation used in DMR publications depending on who is the lead author. The following notation is equivalent

$$
\begin{aligned}
\Delta T_{\ell}^{2} & =T_{\ell}^{2}=\frac{1}{4 \pi} \sum_{m=-\ell}^{\ell} b_{\ell m}^{2}=\frac{1}{4 \pi} \sum_{m=-\ell}^{\ell}\left|a_{\ell m}\right|^{2} \\
\left.<\Delta T_{2}^{2}\right\rangle & =\left\langle Q_{R M S}^{2}>\right.
\end{aligned}
$$

and $Q_{r m s-P S}^{2}$ is an estimate of $\left\langle Q_{R M S}^{2}\right\rangle$. The beam pattern expanded in a Legendre polynomial expansion has been expressed both as $W_{\ell}$ and as $\dot{G}_{\ell}$. 


\section{Appendix D}

\section{Literature Notation}

This Appendix has been compiled to ease comparison between the notation for the correlation function and power spectrum used in this thesis, the DMR publications and the rest of the literature in this field. See Scaramella and Vittorio 1990 for a less comprehensive compilation. If the sky temperature $T(\theta, \phi)=\sum a_{\ell m} Y_{\ell m}(\theta, \phi)$ then using the addition theorem of spherical harmonics (Appendix $\mathrm{H}$ ) we can express the correlation function as a Legendre polynomial expansion with coefficients denoted by a variety of symbols (Table D.1).

In DMR notation, the correlation function has the dimensions of temperature squared $C(\alpha) \equiv<\delta T, \delta T>$ while the others are dimensionless $C(\alpha) \equiv<\delta T / T_{0}, \delta T / T_{0}>$. It should be noticed that the $a_{\ell}^{2}$ of Abbot and Wise is not equal to Peebles' $a_{\ell}^{2}$. When we write $a_{\ell}^{2}$ we are using Peebles' notation. Notice that $C_{\ell}$ is not squared. A shift in Bond's notation should be noticed: Bond et al. (1991) has $C_{\ell}=<\left|a_{\ell m}\right|^{2}>$ while Bond (1994) has $C_{\ell}=C_{T_{\ell}}=\ell(\ell+1)<\left|a_{\ell m}\right|^{2}>/ 2 \pi$ Some authors do not distinguish clearly between the observed quantities in our horizon and the ensemble averaged quantities of a model.

The relation between the various power spectra is

$$
\frac{\leq \Delta T_{\ell}^{2}>}{T_{0}^{2}}=\frac{2 \ell+1}{4 \pi} C_{\ell}=\frac{2 \ell+1}{4 \pi} a_{\ell}^{2}=\left(\frac{\Delta T}{T_{0}}\right)_{\ell}^{2}=\frac{2 \ell+1}{4 \pi}<\left|a_{\ell m}\right|^{2}>=\frac{2 \ell+1}{4 \pi} \frac{2 \pi^{2}}{\ell(\ell+1)} \epsilon_{H}^{2}
$$

For the important $\ell=2$ case which is often used to normalize we have .

$$
\frac{\left\langle\Delta T_{2}^{2}>\right.}{T_{0}^{2}}=\frac{Q_{r m s-P S}^{2}}{T_{0}^{2}}=\frac{5}{4 \pi} C_{2}=\frac{5}{4 \pi} a_{2}^{2}=\left(\frac{\Delta T}{T_{0}}\right)_{2}^{2}=\frac{5}{4 \pi}<\left|a_{2, m}\right|^{2}>=\frac{5}{4 \pi} \frac{\pi^{2}}{3} \epsilon_{H}^{2} .
$$

For the primordial density power spectrum

$$
P(k)=A k^{n}
$$


Table D.1: Diversity of Correlation Function Notation

\begin{tabular}{|llll|}
\hline Observed $^{\alpha}$ & Observed $^{b}$ & Theoretical $^{c}$ & Reference \\
\hline$\frac{1}{4 \pi} \sum^{d}\left|b_{\ell m}\right|^{2} P_{\ell}^{e}$ & $\sum \Delta T_{\ell}^{2} P_{\ell}$ & $\sum<\Delta T_{\ell}^{2}>P_{\ell}$ & DMR publications \\
\hline$\frac{1}{4 \pi} \sum\left|a_{\ell m}\right|^{2} P_{\ell}$ & $\frac{1}{4 \pi} \sum(2 \ell+1) C_{\ell} P_{\ell}$ & $\frac{1}{4 \pi} \sum(2 \ell+1) C_{\ell} P_{\ell}$ & Bond, Efstathiou \\
\hline$\frac{1}{4 \pi} \sum\left|a_{\ell m}\right|^{2} P_{\ell}$ & $\frac{1}{4 \pi} \sum(2 \ell+1) a_{\ell}^{2} P_{\ell}$ & $\frac{1}{4 \pi} \sum(2 \ell+1) a_{\ell}^{2} P_{\ell}$ & Peebles \\
\hline$\frac{1}{4 \pi} \sum\left|a_{\ell m}\right|^{2} P_{\ell}$ & $\frac{1}{4 \pi} \sum(2 \ell+1) \Sigma_{\ell}^{2} P_{\ell}$ & $\frac{1}{4 \pi} \sum<Q_{\ell}^{2}>P_{\ell}$ & Scaramella, Vittorio \\
\hline$\frac{1}{4 \pi} \sum\left|a_{\ell m}\right|^{2} P_{\ell}$ & $\frac{1}{4 \pi} \sum a_{\ell}^{2} P_{\ell}$ & $\frac{1}{4 \pi} \sum(2 \ell+1) \epsilon_{H}^{2} \frac{2 \pi^{2}}{\ell(\ell+1)} P_{\ell}$ & Abbot,Wise, Schaeffer \\
\hline$\frac{1}{4 \pi} \sum\left|a_{\ell m}\right|^{2} P_{\ell}$ & $\frac{1}{4 \pi} \sum(2 \ell+1)\left|a_{\ell m}\right|^{2} P_{\ell}$ & $\frac{1}{4 \pi} \sum(2 \ell+1)<\left|a_{\ell m}\right|^{2}>P_{\ell}$ & Kolb \& Turner \\
\hline$\frac{1}{4 \pi} \sum\left|a_{\ell m}\right|^{2} P_{\ell}$ & $\sum\left(\frac{\Delta T}{T_{o}}\right)_{\ell} P_{\ell}$ & $\sum<\left(\frac{\Delta T}{T_{o}}\right)_{\ell}>P_{\ell}$ & Russians \\
\hline$\frac{1}{4 \pi} \sum\left|a_{\ell m}\right|^{2} P_{\ell}$ & $\sum \sigma_{T_{\ell}}^{2} P_{\ell}$ & $\sum \sigma_{T_{\ell}}^{2} P_{\ell}$ & Adams92 \\
\hline
\end{tabular}

a Observed refers to our horizon volume, this first column is expressed in terms of the spherical harmonic coefficients and the sum is over $\ell$ and $m$.

$b$ This second Observed column is in terms of the power spectrum and the sum is over $\ell$ only.

c Theoretical or ensemble average over all horizon volumes

${ }^{d}$ Sum is over the explicit indices, e.g., $\sum=\sum_{\ell=\ell_{\min }, \ell_{\max }}\left(\sum_{m=-\ell}^{+\ell}\right)$

${ }^{e} P_{\ell} \equiv P_{\ell}(\cos \alpha) W_{\ell}^{2}$ where $W_{\ell}^{2}=e^{-\ell(\ell+1) / \ell_{b}^{2}}$ or $W_{\ell}^{2}=G_{\ell}^{2}$ from Wright et al. 1993 .

one obtains the radiation power spectrum (equation (4.10))

$$
\Delta T_{\ell}^{2}=\left(\frac{2 \ell+1}{4 \pi}\right) \frac{A}{16}\left(\frac{H_{o}}{c}\right)^{n+3} \frac{\Gamma(3-n)}{\Gamma^{2}\left(\frac{4-n}{2}\right)} \frac{\Gamma\left(\ell+\frac{n-1}{2}\right)}{\Gamma\left(\ell+\frac{5-n}{2}\right)} .
$$

For the $n=1$ case the relation between normalization factors $\left(A, H_{0}, \epsilon_{H}\right)$ is

$$
A=\left(\frac{96 \pi^{2}}{5}\right)\left(\frac{c}{H_{o}}\right)^{4} \frac{Q_{r m s-P S}^{2}}{T_{o}^{2}}=8 \pi^{3}\left(\frac{c}{H_{o}}\right)^{4} \epsilon_{H}^{2} .
$$




\section{Appendix E}

\section{Software Versions: Pass 1 vs Pass}

2

Currently there are two incarnations of the software: pass 1 and pass 2. Pass 1 produced the first year maps that were released in June 1993. The pass 2 software was used for the 2 year results (Bennett et al. 1994, Wright et al. 1994, Górski et al. 1994). The 2 year maps were released in June 1994.

The one year released ecliptic coordinate maps were made with "pass 1 " software processing. The 2 year results are based on the upgraded "pass 2 " software processing. Pass 1 and pass 2 software processing are contrasted in Table E.1. The most noticeable difference is the Moon cut angle. Pass 2 corrects for Moon emission when the Moon is $>21^{\circ}$ from a beam center. The pass 1 correction is $>25^{\circ}$. The data processing speed was increased due to the 7 to 4 block data structure conversion. Figures E.1 and E.2 are 2 year sky coverage maps from 53A for pass 1 and pass 2. The decreased Moon cut is primarily responsible for the increase in the number of observations in pass 2 however the overall pattern is essentially the same. Figure E. 3 compares the Yr1 channel auto-correlation functions from pass 1 and pass 2. The differences are small. On October 4, 1991 the 31B channel noise increased dramatically and we do not use $31 \mathrm{~B}$ data after this date. 
Table E.1: Pass 1 versus Pass 2 Processing Software

\begin{tabular}{|c|c|c|}
\hline Quality & Pass 1 & Pass 2 \\
\hline 1 Yr Data ${ }^{a}$ & $89356-90355^{6}$ & $89356-90355$ \\
\hline 2Yr Data & $90356-91355$ & $90356-91355^{c}$ \\
\hline Data type & 7 block $^{d}$ & 4 block \\
\hline outlier cut & $>5 \sigma$ & $>5 \sigma$ \\
\hline data used $(53 \& 90)$ & $87 \%$ & $90 \%$ (more moon, less eclipse) \\
\hline Moon cut & $25^{\circ}(5 \%$ of data $)$ & $21^{\circ}(4.5 \%$ of data $)$ \\
\hline Earth cut $(53 \& 90)$ & $1^{\circ}$ below shield or higher & $1^{\circ}$ below shield or higher \\
\hline Earth cut(31) & $3^{\circ}$ below shield or higher & $3^{\circ}$ below shield or higher \\
\hline$N_{\max } / N_{\min }(53 \& 90) \mathrm{Yr} 1$ & 4.7 & 4.3 \\
\hline$N_{\max } / N_{\min }(31) \mathrm{yr} 1$ & 7.4 & 6.9 \\
\hline$N_{\max } / N_{\min }(53 \& 90) 2 \mathrm{YI}$ & 4.1 & 3.8 \\
\hline$N_{\max } / N_{\min }(31) 2 Y_{I}$ & 6.6 & 6.7 \\
\hline Galactic flag & $10^{\circ}$ & $15^{\circ}$ \\
\hline coordinate system & Galactic & Galactic \\
\hline map resolution & 6 & split $6 / 7 ; 7|b|<20^{\circ}$ \\
\hline Pixel number (size) & $6144\left(2.6^{\circ}\right)^{2}$ & $4032\left(2.6^{\circ}\right)^{2}+2112^{*} 4\left(2.6^{\circ} / 4\right)^{2}$ \\
\hline baseline & $50 \min$ (cubic spline) & $206 \min =2$ orbits, running mean \\
\hline satellite velocity & corrected & corrected \\
\hline Earth velocity & corrected & corrected \\
\hline Sun Velocity & not corrected & corrected $^{e}$ \\
\hline Moon emission & corrected $\left(>25^{\circ}\right)$ & corrected $\left(>21^{\circ}\right)$ \\
\hline Magsus $^{f}$ & corrected $\left(\right.$ fit $\left.^{g}\right)$ & corrected (fit) \\
\hline Memory $^{h}$ & not corrected & corrected \\
\hline Planets & Jupiter corrected & Jupiter, Saturn, Mars corrected \\
\hline Mean $^{i}$ & removed (fit) & removed (fit) \\
\hline
\end{tabular}

"Launch was Nov 18, 1989 or "89322", 332nd day of 1989

${ }^{b}$ December 22, 1989 to December 21, 1990, released in June 1993

${ }^{c}$ minus the eclipse season cut (May 21-July 24), released in summer 1994

${ }^{d}$ refers to the size of the data record structures, the 7 to 4 block conversion saved disk space and lowered the processing time

$e$ Nominal Dipole removed: $3.325 \mathrm{mK}$ in direction J2000 RA= $11^{h} 12^{m} 57^{s} .6$, Dec $=$ $-6^{\circ} 0^{\prime} 36^{\prime \prime}$

$f$ magnetic susceptibility, Chapter 2

${ }^{g}$ See Chapter 3 for difference between corrected and fit

$h$ also known as the "LIA" effect, see Section 2.4

${ }^{i}$ Mean of time ordered data was fitted and removed 

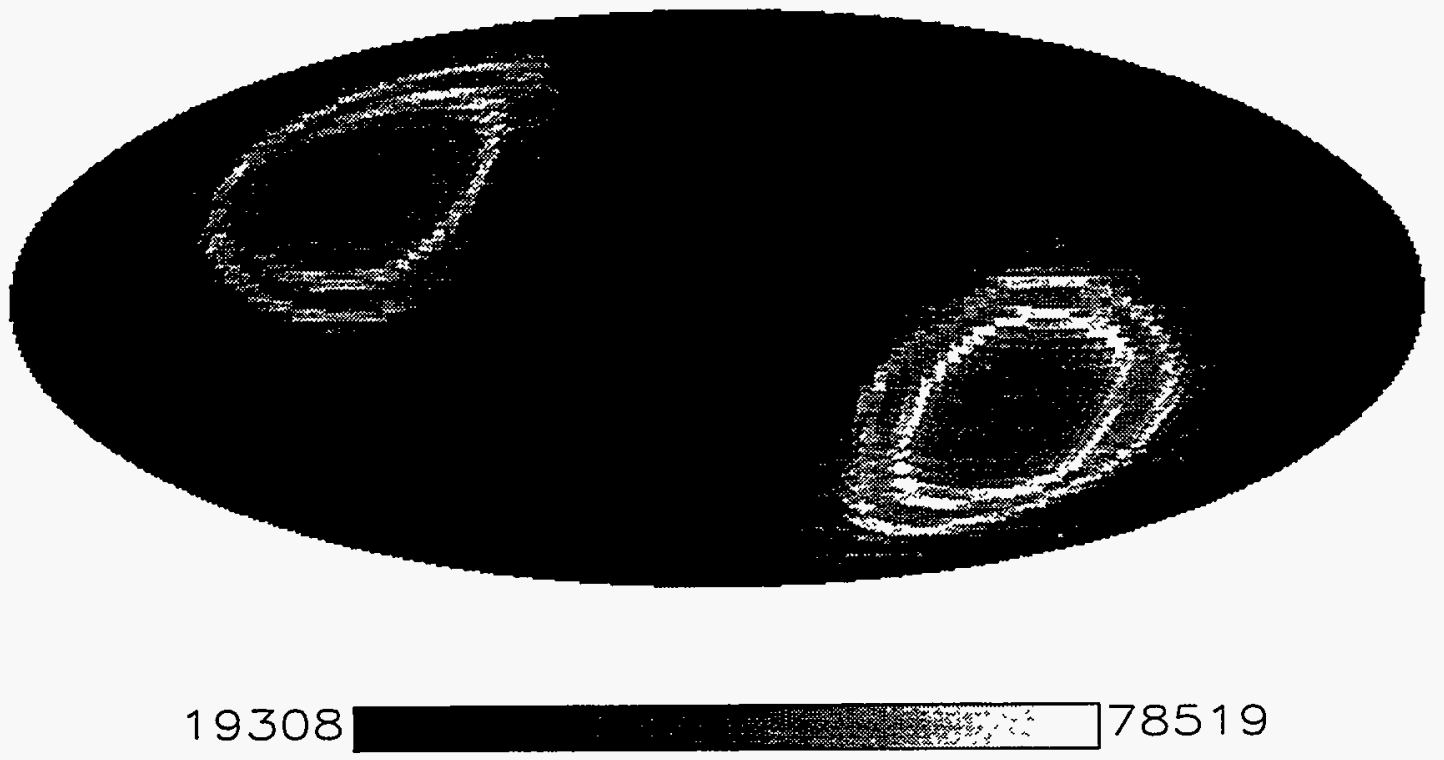

Figure E.1: Pass 1, 53A 2Yr Sky Coverage

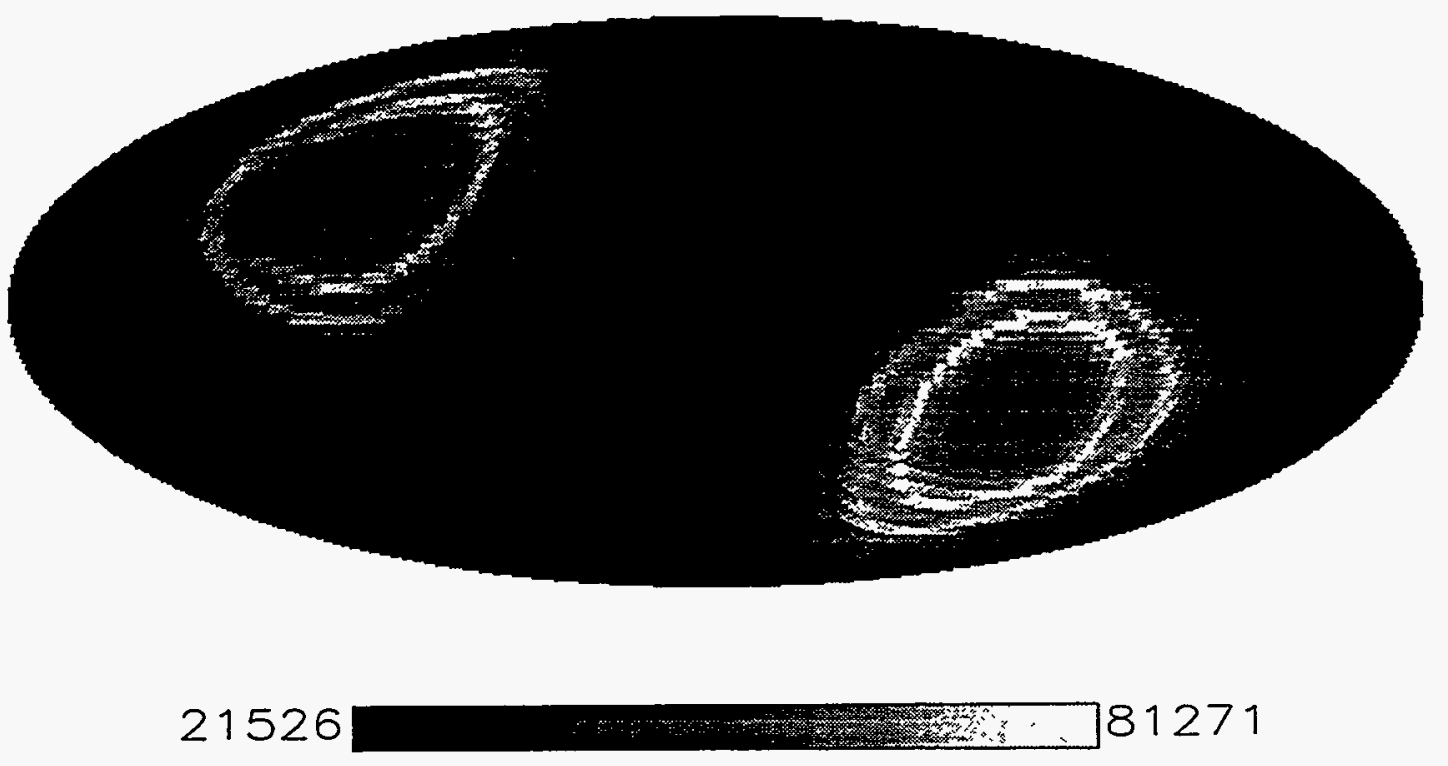

Figure E.2: Pass 2 53A 2Yr Sky Coverage 
Separate Channels 1990 Pass1 vs 1990 Pass2

Channel A
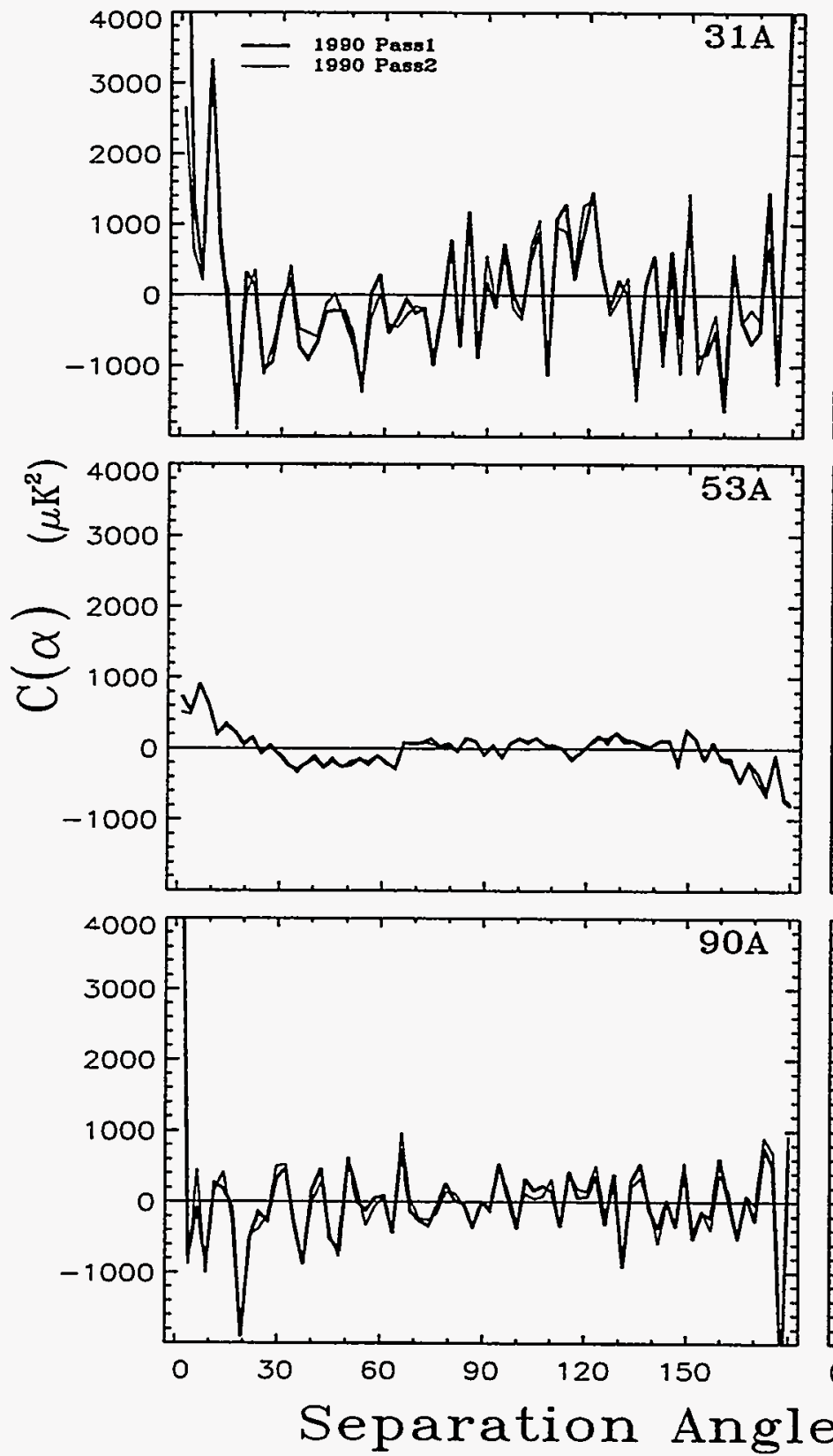

Channel B
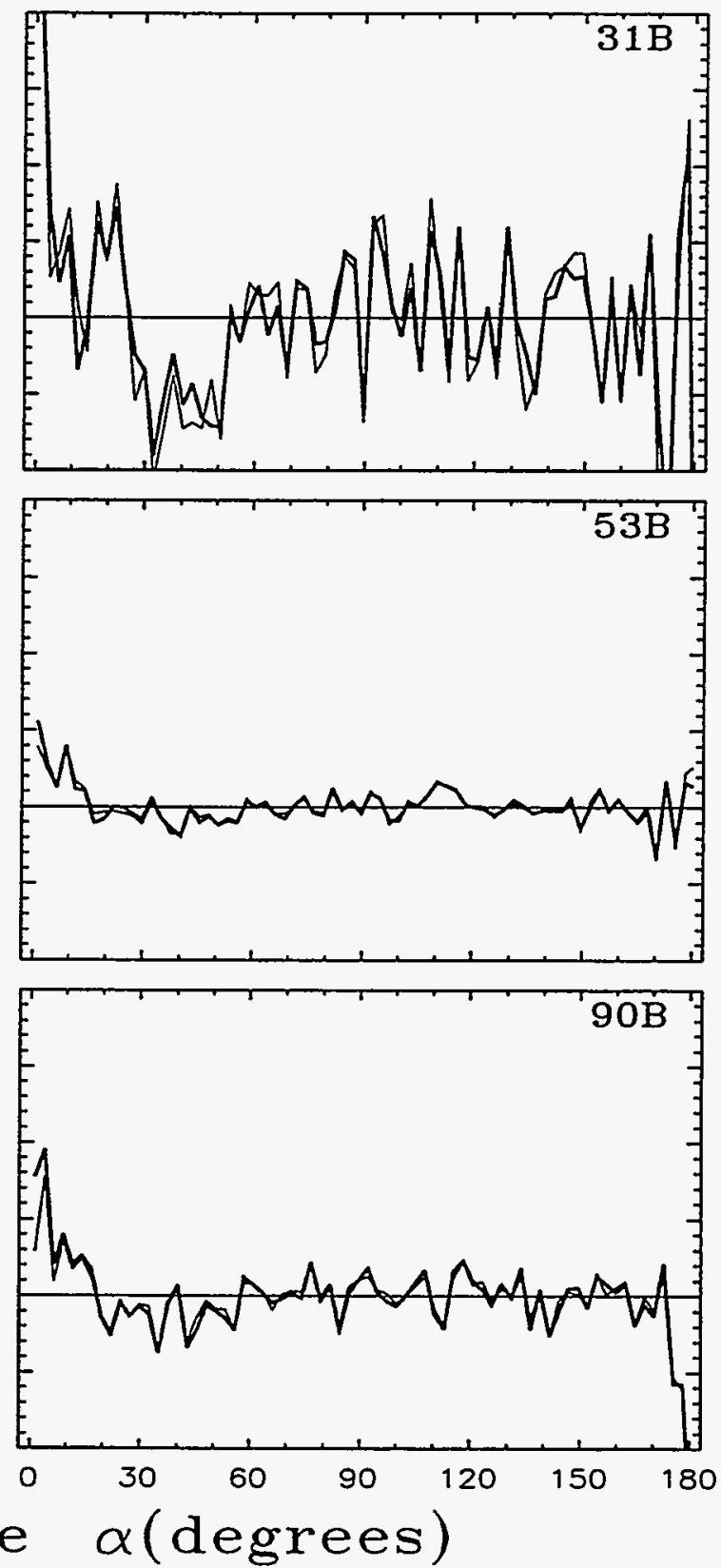

Figure E.3: Separate Channels for Yr1 Pass 1 vs Pass 2. The amount of variation is a small fraction of the noise error bars. $31 \mathrm{GHz}$ shows the largest pass1/pass2 difference. These weighted auto-correlations are for $|b|>20^{\circ}$ and have had the best fit mean, dipole and quadrupole removed. 


\section{Appendix $\mathbf{F}$}

\section{Friedmann Equation}

Einstein's equations of general relativity provide a theoretical framework capable of describing the dynamics of the Universe. Friedmann's equation is a solution to Einstein's equations for an isotropic and homogenous universe and is a differential equation relating the time dependence of the scale factor $R$ to the matter content of the Universe. A useful formulation which explicitly expresses the $R$ dependence of various types of mass density is:

$$
H^{2}=H_{o}^{2}\left[\Omega_{r e l, 0} R^{-4}+\Omega_{n r e l, 0} R^{-3}+\Omega_{c u r v, 0} R^{-2}+\Omega_{v a c, 0} R^{0}\right]
$$

where the $\Omega_{i, 0}$ 's are the present dimensionless densities in units of the present critical density for relativistic, non-relativistic particles, curvature and vacuum densities respectively. Specifically $\Omega_{i, 0} \equiv \frac{\rho_{i, 0}}{\rho_{c, 0}}$ where $\rho_{i} c^{2}$ is the energy density of the $i$ th component and $\rho_{c} c^{2}$ is the critical energy density $\left(=8 \pi G c^{2} / 3 H^{2}\right) \cdot \Omega_{c u r v, 0}=\frac{-k c^{2}}{H_{0}^{2}}$ where the curvature constant $k=+1,-1,0$ for a closed, open and Euclidean universe. The vacuum energy density $\Omega_{v a c, 0}=\frac{\Lambda c^{2}}{3 H_{0}^{2}}$ and $\Lambda$ is the cosmological constant. $\Omega_{0} \equiv \Omega_{r e l, 0}+\Omega_{n r e l, 0}+\Omega_{v a c, 0}$ and $\Omega_{0}=1-\Omega_{\text {curv, } 0}$ (Bond 1993). In a flat universe, $k=0$ and $\Omega_{0}=1$. For a closed universe, $k=+1$, and $\Omega_{0}>1$. For an open universe, $k=-1$, and $\Omega_{0}<1$. The initial ingredients for all cosmological models are the $\Omega_{i}$ parameters. The popular Einstein-deSitter model has no cosmological constant and is flat: $\Lambda=0$ and $k=0$. In the matter dominated epoch the Friedmann equation (F.1) then simplifies to

$$
H^{2}-\frac{8 \pi G}{3} \rho_{n \tau e l}=0
$$

and can be understood as a statement of energy conservation. $E_{k i n}-E_{\text {pot }}=0$. 


\section{Appendix G}

\section{Antenna Temperature}

The spectral brightness of a blackbody is a function of only one parameter, the temperature

$$
B_{\nu}(T)=\frac{2 h \nu^{3}}{c^{2}} \frac{1}{e^{x}-1}
$$

where $x=h \nu / k T$. In the Rayleigh-Jeans region $x<<1$ and thus

$$
B_{\nu}(T)=\frac{2 \nu^{2}}{c^{2}} k T \text {. }
$$

The generalization of equation (G.2) to any $x$ defines the antenna temperature of a blackbody

$$
B_{\nu}(T)=\frac{2 \nu^{2}}{c^{2}} k T_{a n t}(\nu)
$$

Rewriting equation (G.3) yields the relation between antenna and thermodynamic temperature

$$
T_{\text {ant }}(\nu)=\frac{h \nu / k}{e^{x}-1}=T \frac{x}{e^{x}-1} .
$$

Notice that in the Rayleigh-Jeans portion of a blackbody spectrum the antenna temperature and the thermodynamic temperature are equal $\left(T_{a n t}=T\right)$.

The antenna temperatures of the $\mathrm{CMB}$, the kinetic dipole and the normalizing quadrupole amplitude $Q_{r m s-P S}$ are plotted in Figure 2.4. Taking the derivative of equation (G.4) one obtains the relation between antenna and thermodynamic temperature differences

$$
\Delta T_{a n t}=\Delta T \frac{x^{2} e^{x}}{\left(e^{x}-1\right)^{2}}
$$

where here $x=h \nu / k T_{0}$. Notice that the temperature difference conversion depends on a knowledge of $T_{o}$ while equation (G.4) does not. Plugging 31.5, 53 and $90 \mathrm{GHz}$ into equation (G.5) with $T_{o}=2.73$, we get the conversion factors $1.026,1.074,1.226$ respectively. 
In the more general case of non-Planckian spectra $I_{\nu}$ we can define an equivalent antenna temperature by

$$
I_{\nu}=\frac{2 \nu^{2}}{c^{2}} k T_{a n t}
$$

which when combined with equation (G.5) yields

$$
\frac{\Delta I}{I_{o}}=\frac{\Delta T_{\text {ant }}}{T_{\text {ant }}}=\frac{\Delta T}{T_{o}} \frac{x e^{x}}{\left(e^{x}-1\right)}
$$

where $I_{o}$ is an isotropic but not necessarily Planckian radiation field as seen by an observer in the rest frame of the field. 


\section{Appendix $\mathrm{H}$}

\section{Spherical Harmonic Coefficients}

\section{$a_{\ell m}$ and $b_{\ell m}$}

We derive an $a_{\ell m}$ crossing relation due to the reality of $\mathrm{CMB}$ temperatures and then define analogous real valued quantities labelled $b_{\ell m}$ which have the desirable qualities of the $a_{\ell m}$. We use the spherical harmonic definition (see e.g. Press et al. 1993 or Jackson 1975)

$$
Y_{\ell, m}(\theta, \phi)=N_{\ell, m} P_{\ell}^{m}(\cos \theta) e^{i m \phi}
$$

where

$$
N_{\ell, m} \equiv \sqrt{\frac{(2 \ell+1)(\ell-m) !}{4 \pi(\ell+m) !}},
$$

and $P_{\ell}^{m}(\cos \theta)$ are the associated Legendre polynomials (Liboff 1986). We will use the following orthonormality and 'crossing' relations:

$$
\begin{gathered}
\int_{4 \pi} d \Omega Y_{\ell^{\prime} m^{\prime}}^{*}(\theta, \phi) Y_{\ell m}(\theta, \phi)=\delta_{\ell, \ell^{\prime}} \delta_{m m^{\prime}} \\
Y_{\ell, m}(\theta, \phi)=(-1)^{m} Y_{\ell,-m}^{*}(\theta, \phi) .
\end{gathered}
$$

One can expand any real or complex valued function of $\theta$ and $\phi$ as a sum of spherical harmonics,

$$
T(\theta, \phi)=\sum_{\ell=0}^{\infty} \sum_{m=-\ell}^{+\ell} a_{\ell m} Y_{\ell m}(\theta, \phi),
$$

where the set of $a_{\ell m}$ 's completely describes $T(\theta, \phi)$. Since $T(\theta, \phi)$ is a real quantity the $a_{\ell m}$ 's are not independent. A crossing relation exists:

$$
a_{\ell,-m}=(-1)^{m} a_{\ell m}^{*}
$$


Proof:

Since, $T(\theta, \phi) \in \Re$ we have

$$
\begin{aligned}
T^{*} & =T \\
\sum_{\ell m} a_{\ell m}^{*} Y_{\ell m}^{*} & =\sum_{\ell m} a_{\ell m} Y_{\ell m} \\
\sum_{\ell m} a_{\ell m}^{*} Y_{\ell m}^{*} & =\sum_{\ell m}(-1)^{m} a_{\ell m} Y_{\ell,-m}^{*} .
\end{aligned}
$$

Convolve both sides with $\int d \Omega Y_{\ell^{\prime} m^{\prime}}$ and use the orthonormality of the $Y_{\ell m}$ 's ( eq H.3) to get

$$
\begin{aligned}
\sum_{\ell m} a_{\ell m}^{*} \int d \Omega Y_{\ell^{\prime} m^{\prime}} Y_{\ell m}^{*} & =\sum_{\ell m}(-1)^{m} a_{\ell m} \int d \Omega Y_{\ell,-m}^{*} Y_{\ell^{\prime} m^{\prime}} \\
\sum_{\ell m} a_{\ell m}^{*} \delta_{\ell, \ell^{\prime}} \delta_{m m^{\prime}} & =\sum_{\ell m}(-1)^{m} a_{\ell m} \delta_{\ell, \ell^{\prime}} \delta_{-m, m^{\prime}} \\
a_{\ell^{\prime} m^{\prime}}^{*} & =(-1)^{-m^{\prime}} a_{\ell^{\prime},-m^{\prime}} .
\end{aligned}
$$

Removing unnecessary primes, multiplying by $(-1)^{m}$ and taking the complex conjugate of both sides one obtains the crossing relation equation (H.6).

Since we want to avoid making measurements of correlated quanities we introduce real orthonormal spherical harmonics $F_{\ell m}$ with independent coefficients $b_{\ell m}$. These are the functions defined in Smoot et al. 1991 except note the typo: $(1-\mathrm{m})$ ! should be $(\ell-m)$ !. Note that the $F_{\ell, m}$ of Wright et al. 1994 differ by a factor of $\sqrt{4 \pi}$ from those used here.

$$
T(\theta, \phi)=\sum_{\ell m} a_{\ell m} Y_{\ell m}(\theta, \phi)=\sum_{\ell m} b_{\ell m} F_{\ell m}(\theta, \phi)
$$

where

$$
F_{\ell m}(\theta, \phi)=k N_{\ell,|m|} P_{\ell}^{|m|}(\cos \theta)\left\{\begin{array}{ll}
\cos (m \phi) & m \geqq 0 \\
\sin (m \phi) & m<0
\end{array}\right\},
$$

where $k=\sqrt{2}$ for $m \neq 0$ and $k=1$ otherwise. The normalization toggle ensures the orthonormality of the $F_{\ell m}$ 's:

$$
\int_{4 \pi} d \Omega F_{\ell^{\prime} m^{\prime}}(\theta, \phi) F_{\ell m}(\theta, \phi)=\delta_{\ell, \ell^{\prime}} \delta_{m m^{\prime}}
$$

Equation (H.13) leads to the following relation between the $a_{\ell m}$ and the $b_{\ell m}$,

$$
\begin{aligned}
b_{\ell m} & =\sqrt{2} \operatorname{Re}\left(a_{\ell m}\right) \text { for } \mathrm{m}>0 \\
b_{\ell m} & =-\sqrt{2} \operatorname{Im}\left(a_{\ell m}\right) \text { for } \mathrm{m}<0 \\
b_{\ell 0} & =a_{\ell 0}
\end{aligned}
$$


from which one obtains

$$
\sum_{m}\left|a_{\ell m}\right|^{2}=\sum_{m} b_{\ell m}{ }^{2} .
$$




\section{Appendix I}

\section{Correlation Function Power}

\section{Spectrum Expansion}

One finds the addition theorem of spherical harmonics in Jackson (p. 101) in the following form

$$
\sum_{m=-\ell}^{\ell} Y_{\ell m}^{*}(\hat{i}) Y_{\ell m}(\hat{j})=\frac{(2 \ell+1) P_{\ell}(\cos \alpha)}{4 \pi}
$$

where the angle $\alpha_{i j}$ between the directions $\hat{i}$ and $\hat{j}$ is fixed; $\alpha_{i j}=\alpha$. Drop the sum on $m$ and consider any one of the $2 \ell+1$ terms on the left hand side. When averaged over all pairs of directions $\hat{i}$ and $\hat{j}$ separated by $\alpha$ one obtains,

$$
<Y_{\ell m}^{*}(\hat{i}) Y_{\ell m}(\hat{j})>\equiv \frac{\sum_{i j} \delta\left(\alpha-\alpha_{i j}\right) Y_{\ell m}^{*}(\hat{i}) Y_{\ell m}(\hat{j})}{\sum_{i j} \delta\left(\alpha-\alpha_{i j}\right)}=\frac{P_{\ell}(\cos \alpha)}{4 \pi} .
$$

When the indices of $Y^{*}(\hat{i})$ are different from those of $Y(\hat{j})$, the sum over all pairs is zero. To generalize to this case, we prime the $Y(\hat{j})$ indices and introduce delta functions,

$$
<Y_{\ell m}^{*}(\hat{i}) Y_{\ell^{\prime} m^{\prime}}(\hat{j})>\equiv \frac{\sum_{i j} \delta\left(\alpha-\alpha_{i j}\right) Y_{\ell m}^{*}(\hat{i}) Y_{\ell^{\prime} m^{\prime}}(\hat{j})}{\sum_{i j} \delta\left(\alpha-\alpha_{i j}\right)}=\frac{P_{\ell}(\cos \alpha)}{4 \pi} \delta_{\ell \ell^{\prime}} \delta_{m, m^{\prime}}
$$

We will use the addition formula of spherical harmonics in this form.

The two point correlation function of a sky map is defined as

$$
C(\alpha)=<\delta T(\hat{i}) \delta T(\hat{j})>
$$

where $\delta T(\hat{i}) \equiv \mathcal{T}_{i}-\bar{T}$ and the average is over all pairs $i j$ with $\alpha_{i j}=\alpha$. Since $\bar{T}=a_{00} Y_{00}$ and $\delta T \in \Re$ we have

$$
\delta T(\hat{i})=\sum_{\ell=1}^{\infty} \sum_{m=-\ell}^{\ell} a_{\ell m} Y_{\ell m}(\hat{i})=\sum_{\ell=1}^{\infty} \sum_{m=-\ell}^{\ell} a_{\ell m}^{*} Y_{\ell m}^{*}(\hat{i}) .
$$


Equation (I.4) then becomes

$$
\begin{aligned}
C(\alpha) & =<\left[\sum_{\ell=1}^{\infty} \sum_{m=-\ell}^{+\ell} a_{\ell m}^{*} Y_{\ell m}^{*}(\hat{i})\right]\left[\sum_{\ell^{\prime}=2}^{\infty} \sum_{m^{\prime}=-\ell^{\prime}}^{+\ell^{\prime}} a_{\ell^{\prime} m^{\prime}} Y_{\ell^{\prime} m^{\prime}}(\hat{j})\right]> \\
& =\sum_{\ell m \ell^{\prime} m^{\prime}} a_{\ell m}^{*} a_{\ell^{\prime} m^{\prime}}<Y_{\ell m}^{*}(\hat{i}) Y_{\ell^{\prime} m^{\prime}}(\hat{j})> \\
& =\sum_{\ell m}\left|a_{\ell m}\right|^{2} \frac{P_{\ell}(\cos \theta)}{4 \pi},
\end{aligned}
$$

where we have used equation (I.3). Taking the ensemble average we obtain

$$
<C(\alpha)>=\frac{1}{4 \pi} \sum_{\ell m}<\left|a_{\ell m}\right|^{2}>P_{\ell}(\cos \alpha)=\sum_{\ell_{\min }}^{\ell_{\max }}<\Delta T_{\ell}^{2}>P_{\ell}(\cos \alpha) .
$$




\section{Appendix $\mathbf{J}$}

\section{Arbitrary Mean Value of Map Solution}

The normal equations we want to solve are (equation (3.7)),

$$
A \vec{T}=\vec{M} \text {. }
$$

Since $\mathbf{A}$ is singular, we need to know the rank and nullity of $\mathbf{A}$ to determine a meaningful solution. Since $A=V^{T} V$, the dimension of the null space of $A$ is the same as that of $V$. Referring to the structure of $\mathrm{V}$ in equation (3.5), one can see that $\mathrm{V} \vec{T}=0$ if and only if $T_{i}-T_{j}=0$ for all $i$ and $j$. Thus the constant vector $(1, \ldots, 1)$ spans the nullspace of both $\mathbf{A}$ and $\mathrm{V}$ and nullity $(\mathbf{A})=\operatorname{nullity}(\mathrm{V})=1$. Therefore solutions of the normal equations are all of the form

$$
\vec{T}=\vec{T}_{p}+\vec{T}_{\text {constant }}
$$

where $\vec{T}_{p}$ is a particular solution and $\vec{T}_{\text {constant }}$ is any element of the nullspace of $\mathbf{A}$. Therefore any two solutions of the normal equations differ only by a mean value in the maps.

How much does adding $\epsilon$ to the diagonals change the solution?

The DMR inversion algorithm breaks the singularity of $\mathrm{A}$ by adding a small positive number $\epsilon$ to the diagonal elements of $\mathrm{A}$.

$$
(\mathrm{A}+\epsilon \mathrm{I}) \vec{T}_{\epsilon}=\vec{M}
$$

We would like to know how much this $\epsilon$ perturbation changes the solution. Using singular value decomposition we can write the symmetric matrix $\mathbf{A}$ as

$$
\mathrm{A}=\mathrm{U} \Lambda \mathrm{U}^{T}
$$


where $\Lambda$ is a diagonal matrix whose diagonal values are the eigenvalues (singular values) $\lambda_{i}^{2}$ of $\mathrm{A}$. $\mathrm{U}$ is an $n$ by $n$ orthonormal matrix whose $i$ th column is the eigenvector of the eigenvalue $\lambda_{i}^{2}$. Thus,

$$
(\mathbf{A}+\epsilon I)=\mathbf{U} \boldsymbol{\Lambda} \mathbf{U}^{T}+\epsilon \mathbf{I}=\mathbf{U}(\boldsymbol{\Lambda}+\epsilon \mathbf{I}) \mathbf{U}^{T}
$$

Plugging equation (J.5) into (J.3) yields,

$$
\vec{T}_{\epsilon}=\mathrm{U}(\Lambda+\epsilon \mathrm{I})^{-1} \mathrm{U}^{T} \vec{M}
$$

A measure of how different $\vec{T}_{\epsilon}$ is from a desired solution $\vec{T}$ is needed. Make an orthonormal transformation expressing $\vec{T}_{\epsilon}$ in the basis given by the columns of $U$

$$
\begin{aligned}
& \overrightarrow{t_{\epsilon}}=(\boldsymbol{\Lambda}+\epsilon \mathbf{I})^{-1} \vec{m} \\
& \overrightarrow{t_{\epsilon}}=\left(\frac{m_{1}}{\lambda_{1}^{2}+\epsilon}, \ldots, \frac{m_{n}}{\lambda_{n}^{2}+\epsilon}\right)^{T},
\end{aligned}
$$

where $\overrightarrow{t_{\varepsilon}}=\mathrm{U}^{-1} \vec{T}_{\epsilon}$ and $\vec{m}=\mathrm{U}^{-1} \vec{M}$. Also $\left|\overrightarrow{t_{\epsilon}}\right|^{2}=\left|\vec{T}_{\epsilon}\right|^{2}$ since orthonormal transformations are norm preserving. Using equation (J.3) we obtain a measure of the difference between $\vec{T}$ and $\vec{T}_{\epsilon}$

$$
\begin{aligned}
\left|\mathbf{A} \vec{T}_{\epsilon}-\mathbf{A} \vec{T}\right|^{2} & =\left|\left(\vec{M}-\epsilon \vec{T}_{\epsilon}\right)-\vec{M}\right|^{2} \\
& =\left|\epsilon \vec{T}_{\epsilon}\right|^{2} \\
& =\epsilon^{2}\left|\vec{t}_{\epsilon}\right|^{2} \\
& =\sum_{i=1}^{n}\left(\frac{\epsilon}{\lambda_{i}^{2}+\epsilon}\right)^{2} m_{i}^{2} .
\end{aligned}
$$

Since the rank of $\mathbf{A}$ is $n-1$ ("rank + nullity $=n$ "), only one of the eigenvalues, $\lambda_{i}^{2}$, is zero. Without loss of generality let $\lambda_{1}^{2}=0$, then

$$
\left|\mathrm{A} \vec{T}_{\epsilon}-\mathbf{A} \vec{T}\right|^{2}=m_{1}^{2}+\sum_{i=2}^{n}\left(\frac{\epsilon}{\lambda_{i}^{2}+\epsilon}\right)^{2} m_{i}^{2} .
$$

Since the $i$ th column of $U$ is the normalized eigenvector corresponding to $\lambda_{i}^{2}$, we have $\mathrm{U}^{-1}{ }_{1, j}=\left(\frac{1}{\sqrt{n}}, \ldots, \frac{1}{\sqrt{n}}\right)$. Therefore,

$$
m_{1}=\frac{1}{\sqrt{n}} \sum_{i=1}^{n} M_{i} .
$$

But

$$
\sum_{i=1}^{n} M_{i}=\sum_{i=1}^{n}\left(\sum_{j=1}^{N_{\text {cbs }}} V_{i j}^{T} D_{j}\right)=0
$$


(see equation (3.7)). Therefore $m_{1}=0$ and the measure of our solution error becomes

$$
\left|\mathbf{A} \vec{T}_{\epsilon}-\mathbf{A} \vec{T}\right|^{2}=\sum_{i=2}^{n}\left(\frac{\epsilon}{\lambda_{i}^{2}+\epsilon}\right)^{2} m_{i}^{2} .
$$

We can make $\epsilon$ arbitrarily small such that $\epsilon<<\lambda_{i}^{2}$ for all $i$. We obtain the desired result

$$
\lim _{\epsilon \rightarrow 0} \vec{T}_{\epsilon}=\vec{T}
$$

where $\vec{T}$ is a solution (equation (J.2)) of the normal equations. 


\section{Appendix K}

\section{Error on the Correlation Function}

We derive the errors on the cross-correlation function of two maps using the standard error-propagation formula. The weighted correlation function is

$$
C(\alpha)=\frac{1}{W} \sum_{i j} \delta\left(\alpha-\alpha_{i j}\right) w_{i} w_{j} T_{o b s, i} T_{o b s, j},
$$

where $T_{o b s, i}$ and $T_{o b s, j}$ are the temperatures of two different maps and $w_{i}$ and $w_{j}$ are their respective weightings. The $\sum_{i j} \delta\left(\alpha-\alpha_{i j}\right)$ is the sum over all pixel pairs $i j$ with separation angle $\alpha_{i j}=\alpha$ and $W \equiv \sum_{i j} \delta\left(\alpha-\alpha_{i j}\right) w_{i} w_{j}$. The standard propagation of errors formula for the dispersion of $C(\alpha)$ is

$$
\sigma_{c}^{2}(\alpha)=\sum_{m=1}^{2 N_{p i x}}\left(\frac{\partial C(\alpha)}{\partial T_{o b s, m}}\right)^{2} \sigma_{m}^{2}
$$

where $N_{\text {pix }}$ is the number of pixels in each map and $\sigma_{m}^{2}=1 / w_{m}$. Notice that the index $m$ runs over all the pixels in both maps. To evaluate this we need the derivative

$$
\frac{\partial C(\alpha)}{\partial T_{o b s, m}}=\frac{1}{W} \sum_{i j} \delta\left(\alpha-\alpha_{i j}\right) w_{i} w_{j}\left(T_{o b s, i} \delta_{m j}+T_{o b s, j} \delta_{m i}\right),
$$

where $m$ can never be equal to both $i$ and $j$ so we cannot combine the two last terms into one term with a coefficient of 2 . We can use the delta functions to reduce the sum if we let the index $k$ run over all the pixels in the map in which $m$ isn't,

$$
\frac{\partial C(\alpha)}{\partial T_{o b s, m}}=\frac{w_{m}}{W} \sum_{k} \delta\left(\alpha-\alpha_{m k}\right) w_{k} T_{o b s, k} .
$$

Squaring this we get

$$
\left(\frac{\partial C(\alpha)}{\partial T_{o b s, m}}\right)^{2}=\frac{w_{m}^{2}}{W^{2}}\left[\sum_{k} \delta\left(\alpha-\alpha_{m k}\right) w_{k}^{2} T_{o b s, k}^{2}+2 \sum_{k} \delta\left(\alpha-\alpha_{m k}\right) w_{k} T_{o b s, k} \sum_{j, j>k} \delta\left(\alpha-\alpha_{m j}\right) w_{j} T_{o b s, j}\right]
$$


where the second term consists of cross-terms which we will ignore since we are primarily interested in the first noise-noise term. Also since $T_{o b s, i}=T_{i}+n_{i}$, the cross terms cancel each other except for the $T_{i} T_{j}$ terms with small $\alpha_{i j}$ (this comes from the large values of $C(\alpha)$ for small $\alpha$ and small $C(\alpha)$ elsewhere). Thus we are left with

$$
\sigma_{c}^{2}(\alpha)=\frac{1}{W^{2}} \sum_{m=1}^{2 N_{p i x}} w_{m} \sum_{k} \delta\left(\alpha-\alpha_{m k}\right) w_{k}^{2} T_{o b s, k}^{2}
$$

where we have used $\sigma_{m}^{2}=1 / w_{m}$. We can write this as

$\sigma_{c}^{2}(\alpha)=\frac{1}{W^{2}}\left[\sum_{m a p 1, m=1}^{N_{p i x}} w_{m} \sum_{m a p 2, k} \delta\left(\alpha-\alpha_{m k}\right) w_{k}^{2} T_{o b s, k}^{2}+\sum_{m a p 2, m=1}^{N_{p i x}} w_{m} \sum_{m a p 1, k} \delta\left(\alpha-\alpha_{m k}\right) w_{k}^{2} T_{o b s, k}^{2}\right]$.

We are free to rename the indices such that in the first sum $m \rightarrow i$ and $k \rightarrow j$ and in the second sum $m \rightarrow j$ and $k \rightarrow i$. Thus we are left with

$$
\sigma_{c}^{2}(\alpha)=\frac{1}{W^{2}} \sum_{i j} \delta\left(\alpha-\alpha_{i j}\right) w_{i} w_{j}\left[w_{i} T_{o b s, i}^{2}+w_{j} T_{o b s, j}^{2}\right] \equiv \sum_{w}\left[w_{i} T_{o b s, i}^{2}+w_{j} T_{o b s, j}^{2}\right],
$$

which is equation (6.2). Since the map temperatures can be represented as a signal plus a noise term, $T_{o b s, i}=T_{i}+n_{i}$ equation(K.8) has the advantage of depending only on the input map temperatures $T_{o b s, i}$. Covariance matrix formulae depend on some assumed signal and are not expressed in terms of $T_{o b s, i}$. Also notice that although we have ignored the crossterms in equation (K.5), equation (K.8) is not just the noise-noise error since

$$
T_{o b s, i}^{2}=T_{i}^{2}+n_{i}^{2}+2 T_{i} n_{i} .
$$

The "conservative" errors (i.e. larger than just the noise-noise term of the covariance matrix) plotted for example in Figure 3 of Smoot et al. 1992, were computed by using

$$
\begin{aligned}
w_{i} & =N_{i} / \sigma_{c h 1}^{2} \\
w_{j} & =N_{j} / \sigma_{c h 2}^{2}
\end{aligned}
$$

in equation (K.8), where the channel dependent rms values $\sigma_{c h}$ are given in Table 2.1. The result is

$$
\sigma_{c}^{2}(\alpha)=\frac{\sum_{i j} \delta\left(\alpha-\alpha_{i j}\right)\left[\sigma_{c h 1}^{2} N_{j} N_{i}^{2} T_{o b s, i}^{2}+\sigma_{c h 2}^{2} N_{i} N_{j}^{2} T_{o b s, j}^{2}\right]}{\left(\sum_{i j} \delta\left(\alpha-\alpha_{i j}\right) N_{i} N_{j}\right)^{2}} .
$$

By contrast the noise-only variances are

$$
\sigma_{c}^{2}(\alpha)=\frac{\sigma_{c h 1}^{2} \sigma_{c h 2}^{2}}{\sum_{i j} \delta\left(\alpha-\alpha_{i j}\right) N_{i} N_{j}}
$$


where we have used equations (K.10) and (K.11) to evaluate the noise-noise term in equation (6.8). The analogous variances for the auto-correlations are the conservative

$$
\sigma_{c}^{2}(\alpha)=4 \frac{\sum_{i j} \delta\left(\alpha-\alpha_{i j}\right) \sigma_{c h}^{2} N_{i} N_{j}^{2} T_{o b s, j}^{2}}{\left(\sum_{i j} \delta\left(\alpha-\alpha_{i j}\right) N_{i} N_{j}\right)^{2}}
$$

and the noise-only

$$
\sigma_{c}^{2}(\alpha)=2 \frac{\sigma_{c h}^{4}}{\sum_{i j} \delta\left(\alpha-\alpha_{i j}\right) N_{i} N_{j}}
$$




\section{Appendix L}

\section{Relation to Density Contrast}

\section{Two-Point Correlation Function}

The derivations of the density contrast correlation function and the temperature correlation function expressed in terms of power spectra are analogous. We use notation to make this parallelism explicit

$$
\begin{gathered}
\delta T(\hat{n})=\sum a_{\ell m} Y_{\ell m}(\hat{n}) \rightarrow C(\alpha)=\langle\delta T(\hat{n}) \delta T(\hat{n}+\alpha)> \\
\delta(\vec{x})=\frac{1}{V} \sum_{\vec{k}} \delta_{k} e^{-i \vec{k} \cdot \vec{x}} \rightarrow \xi(\vec{r})=\langle\delta(\vec{x}) \delta(\vec{x}+\vec{r})\rangle_{\vec{x}}
\end{gathered}
$$

where the angle brackets in equation (L.1) denote an average over all directions $\hat{n}$ and for each $\hat{n}$ an average over all directions which are $\alpha$ degrees from $\hat{n}$.

$$
\begin{aligned}
C(\alpha) & =\left\langle\left(\sum a_{\ell m} Y_{\ell m}(\hat{n})\right)\left(\sum a_{\ell^{\prime} m^{\prime}}^{*} Y_{\ell^{\prime} m^{\prime}}^{*}(\hat{n}+\alpha)\right)\right\rangle \\
\xi(\vec{r}) & \left.=\frac{1}{V^{2}}<\left(\sum_{\vec{k}} \delta_{\vec{k}} e^{-i \vec{k} \cdot \vec{x}}\right)\left(\sum_{\vec{k}^{\prime}} \delta_{\vec{k}^{\prime}}^{*} e^{+i \hat{k}^{\prime} \cdot(\vec{x}+\vec{r})}\right)\right\rangle_{\vec{x}} \\
C(\alpha) & =\sum_{\ell \ell^{\prime} m m^{\prime}} a_{\ell m} a_{\ell^{\prime} m^{\prime}}^{*}\left\langle Y_{\ell m}(\hat{n}) Y_{\ell^{\prime} m^{\prime}}^{*}(\hat{n}+\alpha)\right\rangle \\
\xi(\vec{r}) & =\frac{1}{V^{2}} \sum_{\vec{k}, \vec{k}^{\prime}} \delta_{\vec{k}} \delta_{\vec{k}^{\prime}}^{*} e^{i \vec{k}^{\prime} \cdot \vec{r}}\left\langle e^{i\left(\vec{k}^{\prime}-\vec{k}\right) \cdot \vec{x}}\right\rangle_{\vec{x}}
\end{aligned}
$$




$$
\begin{aligned}
C(\alpha) & =\frac{1}{4 \pi} \sum_{\ell m}\left|a_{\ell m}\right|^{2} P_{\ell}(\cos \alpha) \\
\xi(\vec{r}) & =\frac{1}{V^{2}} \sum_{\vec{k}}\left|\delta_{\vec{k}}\right|^{2} e^{i \vec{k} \cdot \vec{r}}
\end{aligned}
$$

Thus the correlation functions are the Fourier transform and Legendre expansion of the power spectra

$$
\begin{aligned}
C(\alpha) & =\sum_{\ell} \Delta T_{\ell}^{2} P_{\ell}(\cos \alpha) \\
\xi(r) & =\int d^{3} k P(k) e^{i \vec{k} \cdot \vec{r}}
\end{aligned}
$$

where $r=|\vec{r}|$, and the power spectrum analogs as a function of $n$ and scale $k(\ell)$ are

$$
\begin{aligned}
P(k) & =A k^{n} \\
\Delta T_{\ell}^{2} & =Q_{r m s-P S}^{2} \frac{2 \ell+1}{5} \frac{\Gamma\left(\ell+\frac{n-1}{2}\right)}{\Gamma\left(\ell+\frac{5-n}{2}\right)} \frac{\Gamma\left(\frac{9-n}{2}\right)}{\Gamma\left(\frac{3+n}{2}\right)},
\end{aligned}
$$

where $A$ and $Q_{\text {rms-PS }}$ are normalization constants. 


\section{Acknowledgements}

I thank my advisor George Smoot for many hours of helpful conversations during which his cryptically simple thought experiments often cleared the statisical jungle into which I had wandered.

I thank my teachers Joe Silk, Marc Davis, Frank Shu, Hy Spinrad, Carl Heiles and Lawrence Hall for teaching me some astrophysics and Eugene Cummins, Orlando Alvarez, Mahiko Suzuki and J. D. Jackson for teaching me some physics.

I thank Karl Fisher for the hours in which we hashed out homework solutions and for his ability to tolerate my often opaque brain dumps. I thank Mario Tafalla, Leewah Yeh and Jim Bartlett for many helpful discussions.

At LBL I thank the theoretical statistician Luis Tenorio for generously sharing batch queues with a guy who doesn't even know what a random variable is. I thank the cultured cognoscente Jon Aymon for nursing a computer fledgling into a fellow bit-tiddler. I thank Chris Witebsky for his bug-ferreting fastidiousness and for keeping all systems go. I thank Michelle Mac-Limon for late night sanity checks and much of my Mac-education. I thank Giovanni De Amici for all kinds of help and Marc Bensadoun for among other things helping Figures 1.1, 1.3, 2.1, and 2.2 see the light of day. I thank Jeanette Larsen for keeping the group office running and for being my only defense against the sucking time-sinks of the bureaucractic abyss.

At the Cosmology Data Analysis Center I thank Gary Hinshaw for his humor, lucidity, and IDL-wizardry. I thank Al Kogut for a model of laconic independence which helped me profit from his former advisor, and for many highly accurate back-handed passes. I thank Phil Keegstra for his dedication and readiness to share his detailed knowledge of the DMR codes. I thank Chuck Bennett and Ned Wright for helpful advice. I thank the other members of the DMR team, Pete Jackson, Vijay Kumar, Tony Banday, Kris Górski, Bob Kummerer, Karen Loewenstein, Jairo Santana, Ed Kaita and Laurie Rokke for their cooperative expertise which helped to produce and interpret the best CMB anisotropy data in the universe.

The COBE project has been supported by the Astrophysics Division of the NASA Office of Space Sciences and Applications. This work was supported by a National Aeronautics

and Space Administration Research Training Grant (NGT-50783) and the University of 


\section{California.}

I dedicate this thesis to my family, but for whom none of this would be possible. 